\title{
UCRL-TR-232090
}

LAWRENCE LIVERMORE N A T IO N A L LABORATORY

J. D. Lindl

June 22, 2007 
This document was prepared as an account of work sponsored by an agency of the United States government. Neither the United States government nor Lawrence Livermore National Security, LLC, nor any of their employees makes any warranty, expressed or implied, or assumes any legal liability or responsibility for the accuracy, completeness, or usefulness of any information, apparatus, product, or process disclosed, or represents that its use would not infringe privately owned rights. Reference herein to any specific commercial product, process, or service by trade name, trademark, manufacturer, or otherwise does not necessarily constitute or imply its endorsement, recommendation, or favoring by the United States government or Lawrence Livermore National Security, LLC. The views and opinions of authors expressed herein do not necessarily state or reflect those of the United States government or Lawrence Livermore National Security, LLC, and shall not be used for advertising or product endorsement purposes.

This work performed under the auspices of the U.S. Department of Energy by Lawrence Livermore National Laboratory under Contract DE-AC52-07NA27344. 
UCRL-TR-232090

$1980 \mathrm{ICF}$

Annual Repost

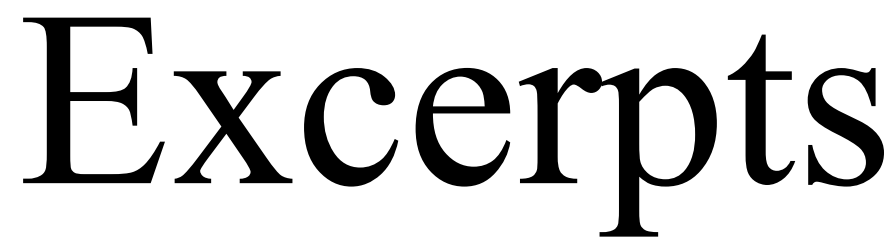


4

$1980 \frac{\text { Laser Program }}{\substack{\text { Annual Report } \\ \text { Excerpts }}}$

Scientific Editors: Lamar W. Coleman

William F. Krupke

Publication Editor: John R. Strack

MS date: December 1981

Lawrence Livermore National Laboratory

University of California - Livermore, CA • 94550 


\section{Excerpts From The 1980 ICF Annual Report}

Pages 2-2 through 2-5

Pages 2-8 through 2-18

Pages 2-20 through 2-26

Pages 2-38 through 2-43

Pages 4-2 through 4-45

Some of these pages have been cited as unpublished references in the Book "Inertial Confinement Fusion" by John D. Lindl. They are reprinted here for the convenience of the reader.

"Inertial Confinement Fusion" citations that pertain to the 1980 Annual Report are:

Chapter 2, references $44,45$. 


\section{Laser Targets}

\section{Hot-Electron Scaling in Hohlraums and Its Impact on Nova Ignition-Capsule Design}

Fig. 2-1. Hot-electron fraction inferred from 50-keV $\times$ rays.

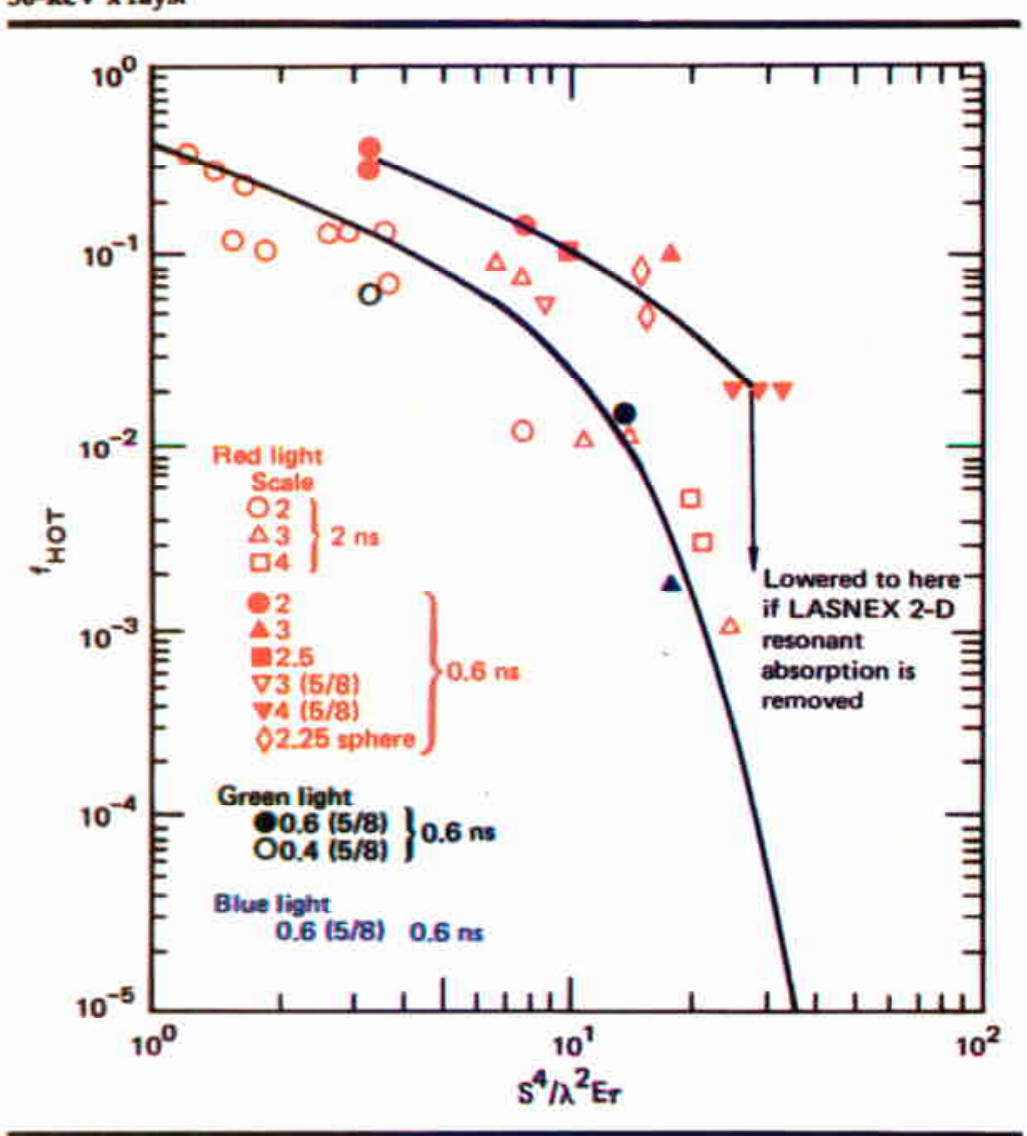

and third harmonics of 1.06- $\mu \mathrm{m}$ light. We also extended our $1.06-\mu \mathrm{m}$ data at $0.6 \mathrm{~ns}$ to include a variety of different hohlraum sizes and geometries. As a unit scale size for our targets, we adopted a Caim target $500 \mu \mathrm{m}$ in diameter and $800 \mu \mathrm{m}$ in length, and we irradiated Caim targets with this ratio of length to diameter in scale sizes up to five times the basic size. We also irradiated a variety of 5/8-scale Caim targets, which had their lengths equal to their diameters, and some spherical hohlraums, whose sizes are specified by their diameter in millimetres. The values of the hot-electron fraction, $\mathrm{f}_{\mathrm{HO}}$, inferred in these experiments are plotted vs $S^{4} / \lambda^{2} E r$ in Fig. 2-1, where $S$ is either the scale size of the hohlraum or the equivalent scale size based on surface area, $\lambda$ is the laser wavelength in micrometres, $\mathrm{E}$ is the laser energy in kilojoules, and $\tau$ is the laser pulse length in nanoseconds. The hotelectron fraction is inferred from the $50-\mathrm{keV}$ FFLEX data, assuming a 50-keV Maxwellian spectrum for the suprathermal electrons. Using the formula of Kruer, ${ }^{1}$ we have $\mathrm{E}_{\mathrm{HOT}}$ in joules, given by

$\mathrm{E}_{\text {HOт }}=\frac{\mathrm{I}_{2}(\mathrm{keV} / \mathrm{keV} / 4 \pi)}{5 \times 10^{11}} \frac{79}{\mathrm{Z}}$,

where $I$, is the $4 \pi x$-ray fluence at an energy equivalent to the temperature of the electron distribution. The temperature of the hot-electron spectrum, as well as the angular distribution of $x$ rays from these hohlraums, is quite uncertain, so the fractions quoted are probably uncertain to at least a factor of 2 . The parameter $\mathrm{S}^{4} / \lambda^{2} \mathrm{E} r$ is a hohlraum plasma-filling scaling 
parameter consistent with LASNEX calculations. If the closing time scales as $\tau_{c}=$ $S / V_{S}$, where $V_{S}$ is the sound velocity of the laser-heated corona and $\tau_{\mathrm{c}}$ is the closure time to some fraction of critical density, and where $V_{S} \sim \sqrt{Z T_{e}}$ and $Z \sim \sqrt{T_{e}}$ for high- $Z$ plasmas, then

$\mathrm{V}_{\mathrm{S}} \sim \mathrm{T}_{\mathrm{e}}^{3 / 4}$.

From flux-limit arguments, the laser intensity, $\mathrm{I}$, is related to the material temperature by

$\mathrm{I} \sim \mathrm{n}_{\mathrm{c}} \mathrm{T}_{\mathrm{e}} \mathrm{v}_{\mathrm{e}} \sim \mathrm{n}_{\mathrm{c}} \mathrm{T}_{\mathrm{e}}^{3 / 2} \sim \frac{\mathrm{T}_{e}^{3 / 2}}{\lambda^{2}}$

Since $I \sim E / S^{2} \tau_{L}$, where $\tau_{L}$ is the laser pulse length, we have

$\mathrm{T}_{\mathrm{e}}^{3 / 2} \sim \frac{\lambda^{2} \mathrm{E}}{\mathrm{S}^{2} \tau_{\mathrm{L}}}$.

Combining Eqs. (2) and (4) gives

$\left(\frac{\tau_{c}}{\tau_{L}}\right)^{2} \sim \frac{S^{4}}{\lambda^{2} E \tau_{L}}$.

Experiments having the same value of $\tau_{d} / \tau_{L}$ will have the same fraction of energy remaining in the pulse when the hohlraum fills to a given density.

If hot-electron production in hohlraums is dominated by plasma phenomena such as
Raman backscatter, $2 \omega_{\text {pe }}$ instability, and filamentation, which occur in large volumes of undendense plasma, then experiments with the same value of $\tau_{\mathrm{d}} / \tau_{\mathrm{L}}$ should have the same hot-electron fraction. The 1.06- $\mu \mathrm{m}$ data at $2 \mathrm{~ns}$, as well as the $2 \omega$ and $3 \omega$ data at $0.6 \mathrm{~ns}$, do lie along a single curve, as seen in Fig. 2-1. The $2 \omega$ and $3 w$ data are for experiments conducted using only 20 to $30 \mathrm{~J}$ of incident energy and must be considered very preliminary; however, the data are indicative of a large reduction in the suprathermal fraction compared to what would be expected with $1.06-\mu \mathrm{m}$ light. The 1.06- $\mu \mathrm{m}$ data at $0.6 \mathrm{~ns}$ also appear to lie on a similar curve, but one that lies consistently above the curve for the 2-ns data. This may be due to an enhanced resonant absorption contribution for the shorterpulse, higher-intensity experiments. A LASNEX two-dimensional calculation of the 5/8-scale 4.0 Caim and a 0.6-ns pulse duration indicates that, if the resonant absorption contribution is removed, the residual flux at $50 \mathrm{keV}$ would be reduced by a factor of 2 and would lie near the 2-ns curve. Similar calculations for the rest of the 0.6-ns experiments remain to be done. Also, the assumption of a constant $T_{H O T}$ is probably incorrect. Recent results obtained using particle-simulation codes ${ }^{2}$ show that $T_{\text {HOT }}$ varies as $\left(\mathrm{D}^{2}\right)^{1 / 3}$ for processes near

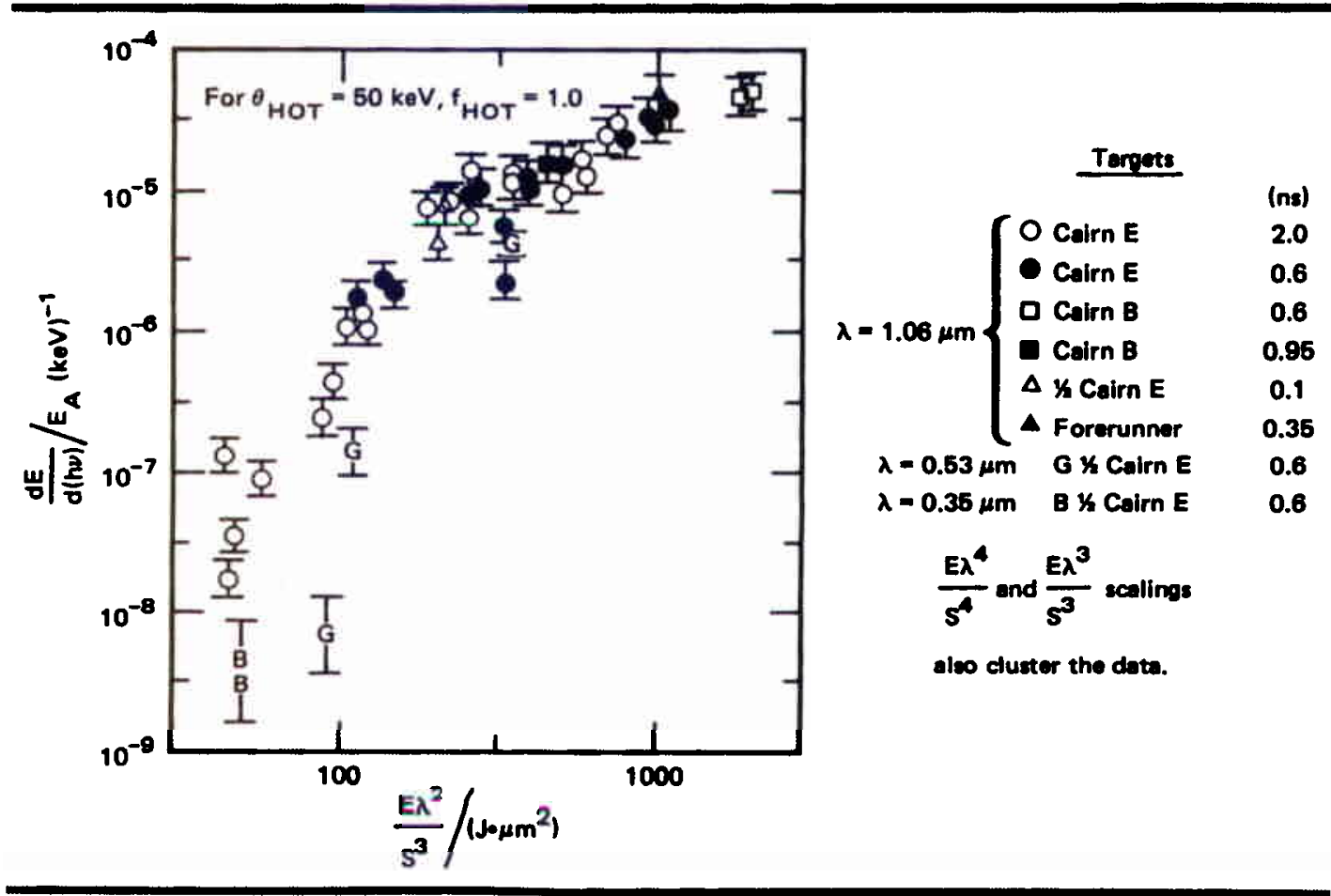

Fig. 2-2 Normalized suprathermal x-ray flux of $50 \mathrm{keV}$ ecales $\approx \mathrm{E}^{2} / \mathrm{S}^{3}$. 
one-quarter $\rho_{c}$ Further experiments are planned at $5 \mathrm{~ns}$ to determine the pulselength scaling of the suprathermal electron production.

Empirically, the 50-keV $x$-ray signal data have less scatter if they are scaled with no pulse-length dependence (see Fig. 2-2). ${ }^{3}$ The best overall fit to the data occurs when they are scaled with $S^{3} / \lambda^{2} E$. We will not obtain a definitive understanding of the processes involved in hohlraums until we can actually measure conditions inside a hohlraum, including density, temperature, and density and velocity gradients. We must also conduct a variety of experiments with long scale lengths to isolate and study specific contributing effects. It should be noted that the electrons that cause serious preheat problems for Nova double-shell targets are those with energies of about $200 \mathrm{keV}$. We have only recently begun to measure $x$ rays of this energy, so we presently have much

Fig 2-3. Maximum radiation temperature ve lawer energy for fixed f

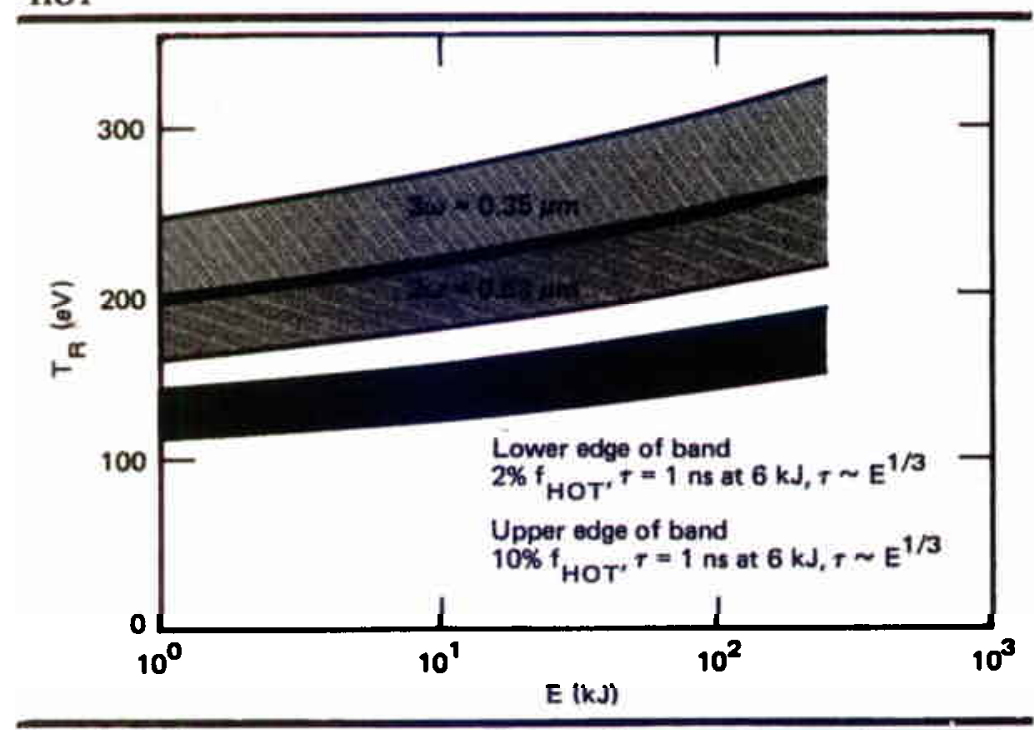

Fig. 2-4. Peak pressure achievable on Nova as a function of wavelength. less scaling information at this energy. Early results, however, indicate that the signals

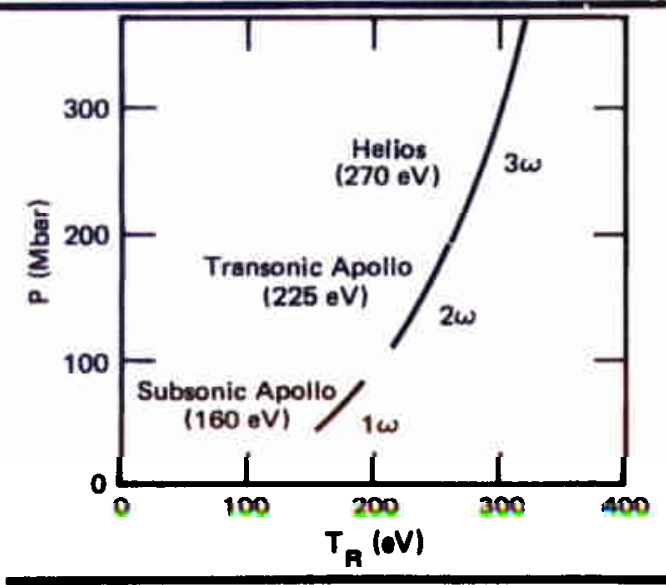

from these electrons scale in essentially the same way as do the $50-\mathrm{keV}$ signals.

The above scaling relation, when combined with the Marshak scaling law for radiation temperature in a hohlraum, allows us to predict the design parameters likely to be available on Nova.

If we scale the laser pulse length to the capsule size to get optimal capsule performance, we have $\tau_{L} \sim E^{1 / 3}$. From the Marshak scaling of hohlraum radiation temperature, $\mathrm{T}_{R}$, we have

$T_{R} \sim\left(\frac{\epsilon E}{S^{2} \tau_{L}^{1 / 2}}\right)^{1 / 3}$

for zonstant conversion efficiency, where $\epsilon$ is the conversion efficiency from laser light to $x$ rays. If we use the hohlraum-filling scaling for hot electrons, we have

$\frac{S^{4}}{\lambda^{2} E_{\tau_{L}}}=$ constant

for constant hot-electron fraction. Combining Eqs. (5) through (7), we get

$T_{R} \sim\left(\frac{\epsilon E^{1 / 6}}{\lambda}\right)^{1 / 3}$.

If, instead, we use the empirical fit to the hot-electron data, we have

$\frac{S^{3}}{\lambda^{2} E}=$ constant ,

which gives

$T_{R} \sim\left(\frac{\epsilon E^{1 / 6}}{\lambda^{4 / 3}}\right)^{1 / 3}$.

One conclusion evident from either Eq. (8) or Eq. (10) is that it is much more effective to shorten the laser wavelength than it is to increase laser energy as a means of increasing the hohlraum temperature. Equations (8) and (10) assume a constant conversion efficiency. If we include the observed increase in conversion efficiency for short wavelength, which scales approximately as $\epsilon \sim \lambda^{-1 / 2}$, the increased temperature available at shorter-wavelength is even greater. Figure 2-3 gives the attainable driving temperature at 1.06, 0.53 , and $0.35 \mu \mathrm{m}$ for different levels of hot electrons as a function of laser energy. Equation (8) was used in producing these curves. The empirical fit to the data, obtained from Eq. (10), would give somewhat higher estimates for the temperature with $2 \omega$ and $3 \omega$ light. Laser entrancehole losses and the symmetry of implosion, 
rather than hot-electron production, may limit hohlraum temperatures for the shorter wavelengths.

The increased range of driving temperatures made available by building Nova with $2 \omega$ and $3 \omega$ capabilities has a large impact on the qualitative nature of the designs that can be tested to produce the implosion velocity, convergence, compression, and fuel temperatures required for D-T ignition. The driving pressure of subsonic ablation scales as the third to fourth power of the radiation temperature. The increased temperatures shown in Fig. 2-3 lead to a factor of 5 to 10 increase in peak implosion pressure as the irradiation wavelength is shifted from 1.06 to $0.35 \mu \mathrm{m}$ at constant hot electron fraction, as shown in Fig. 2-4.

Using 1.06- $\mu \mathrm{m}$ light, we should be able to generate a peak implosion pressure of 40 to $80 \mathrm{Mbar}$. This pressure can drive the $160 \mathrm{eV}$ Apollo capsule shown in Fig. 2-5(a) to ignition conditions. However, the high convergence and subsonic nature of the implosion require satisfaction of extremely severe tolerances on the uniformity of radiation drive.

Many features of the $160-\mathrm{eV}$ Apollo capsule present extremely difficult fabrication problems. High-quality, free-standing high- $\mathrm{Z}$ shells in this size range have never been formed before. Also, even with $100-\AA$ surface finishes, the ARTIC code RayleighTaylor calculations predict near penetration of 6:1 aspect-ratio gold shells. Hence, the

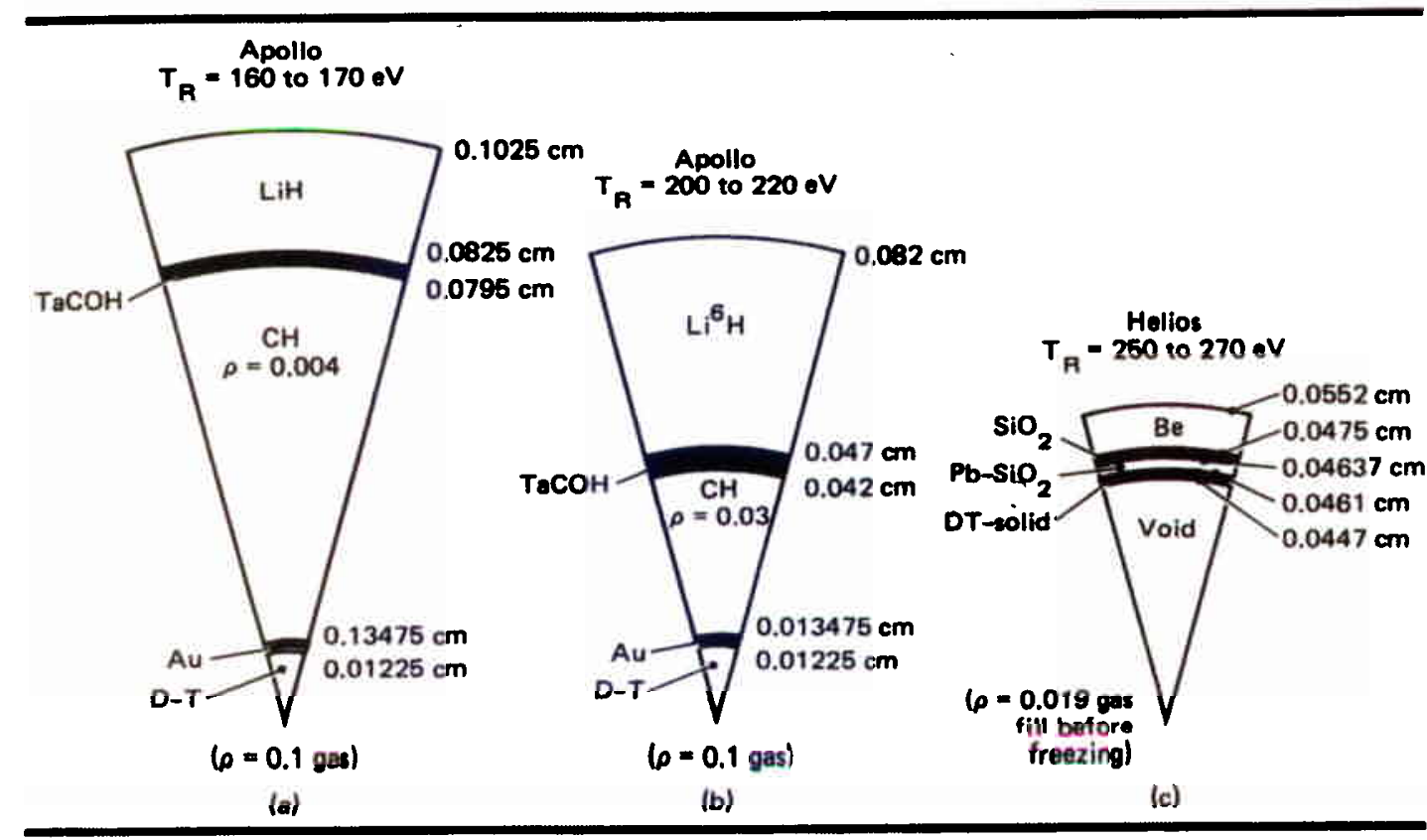

inner shell will probably require a graded-Z, graded-density composition to reduce the Atwood number on its outer surface. The inner shell may also require a low- $Z$ liner on its inside surface to eliminate a mix of high- $Z$ material with the D-T fuel.

Author: J. D. Lindl
Fig. 2-5. Ponsible designs for Nova ignition capoules. 
High-Density Cryogenic Targets for Shiva and Novette

In designing capsules for large-hohlraum Shiva experiments, we found that simple glass-and-parylene cryogenic targets perform extremely well in our nominal 
hohlraum environment. LASNEX simulations indicate that these targets might attain average D-T fuel densities ranging from 150 to 250 times solid density on Shiva and yield $10^{7}$ neutrons. (Normal solid density of $D-T$ is $0.21 \mathrm{~g} / \mathrm{cm}^{3}$.) When we change the model to one that we believe better simulates Novette hohlraums, we find that these same targets might reach average fuel densities of 1500 times liquid density $(1500 \times)$.

This article will discuss why these very simple cryogenic targets can approach $1000 \times$ and will examine some of the effects that may make the targets fall short of the mark. At the end of the article, we will provide some performance predictions for these targets on Shiva and Novette.

The Targets. Figure 2-12(a) shows the type of target we have been examining. The capsule itself is a simple glass shell, $200 \mathrm{\mu m}$ in radius and $5 \mu \mathrm{m}$ thick, coated with a parylene $(\mathrm{CH})$ ablator to a thickness of 20 to $30 \mathrm{~mm}$. The fuel in these capsules takes the form of a thin $(\sim 1.6 \mu \mathrm{m})$ cryogenic layer of $D-T$, which is obtained by freezing out the $0.01-\mathrm{g} / \mathrm{cm}^{3} \mathrm{D}-\mathrm{T}$ gaseous fill.

Figure 2-12(b) shows the cryogenic hohlraum. The support pylon provides a cold jet of He gas, which flows through two $100-\mu \mathrm{m}$ holes, cools the capsule, and then flows out of the hohlraum. The hohlraum greatly simplifies the task of fielding cryogenic targets, since it shields the capsule from room-temperature radiation that might damage the uniform cryogenic layer. The He jet should not affect the laser-hohlraum physics because its density, about $10^{16}$ atoms $/ \mathrm{cm}^{3}$, is well below critical density.
Pieces of the Puzzle. While running an extensive design series, we made several observations about the target's performance, some of which appeared rather strange. Examination of the various observations has led to an understanding of what is important in making glass and $\mathrm{CH}$ tangets attain high density. Some of the more important observations are

- If we calculate the performance of one of our targets with gas fill, it attains 30 to $100 \times$. Freezing out the gas raises the peak average density of the target by about a factor of 10 .

- A hot electron fraction, $\mathrm{f}_{\mathrm{HO}}$, greater than $1 \%$ degrades the performance of the target. Figure 2-13 plots peak density vs $f_{\text {HOr }}$ from one-dimensional simulations of these targets. Note that, even at $\mathrm{f}_{\mathrm{HOT}} \approx$ $10 \%$, the targets can conceivably exceed $200 \times$.

- For a given $f_{\text {HOT }}$, the peak fuel density is relatively independent of when the hot electrons appear or what pulse shape they may have. The only thing that matters is how many hot electrons are produced. This is shown in Fig. 2-14, which plots $\rho_{\max }$ vs $t_{\text {HOT }}$ where $t_{\text {HOT }}$ is the time at which we applied $3 \% \mathrm{f}_{\mathrm{HOT}}$ with a 50-ps FWHIM.

- Targets that have grossly preheated pushers $(>20 \mathrm{eV})$ due to $x$ rays can still exceed $1000 \times$ if $f_{H O r}$ does not exceed the level expected with the Novette laser driver $(<1 \%)$.

- A related observation is that, in the highest-density targets, the peak fuel pressure can be substantially greater than the

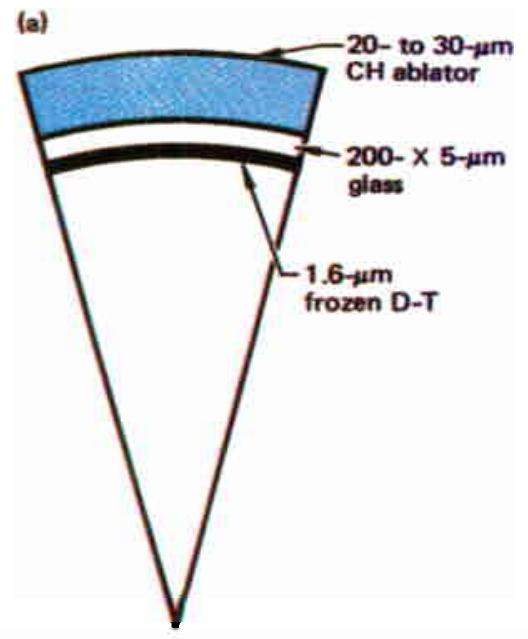

(b)

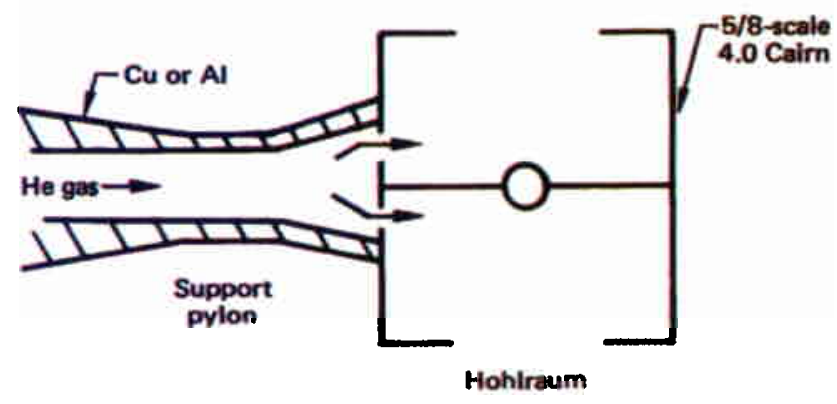

Fig. 2-12, (a) Cryosenic copoule. (b) Cryogenic 5/8-scale 4.0 Cairn. 
stagnation pressure of the hydrodynamic system without fuel. For example, the grossly preheated system we mentioned above achieved a fuel pressure of 4 jerk $/ \mathrm{cm}^{3}$. Without the fuel, the peak stagnation pressure is $0.5 j \mathrm{jer} / \mathrm{cm}^{3}$.

- A very strange result was that the very highest density was attained when we tried to degrade the calculated performance. We were trying to save computer time on two-dimensional calculations by mocking up the effects of $3 \%$ electron preheat with an energy source in the pusher. These implosions (which had expanded, spongy pushers) resulted in peak densities of $2000 \times$ and average densities of $\approx 1500 \times$.

Unifying Theory. All the observations described above can be explained by a two-part hydrodynamic theory of the capsules. The first part of the theory is that it is both possible, and necessary, to keep some regions of the fuel on a very low adiabat. In one-dimensional calculations, increases or decreases in the peak fuel density come principally from processes that affect the adiabat of the coldest part of the fuel.

The second part of the theory is that, in achieving the very highest densities

Fig. 2-13. Calculated peak density as a function of hotelectron fraction for a 200- $\times$ 5-um target with a 20-jm coating of $\mathrm{CH}$.

Fig, 2-14. Peak density achieved by cryogenic targets. (a) Peak fuel density vs time we applied 240 J of hot electrons (3\%) in 50 ps. (b) Inner radius of the pusher vs time.

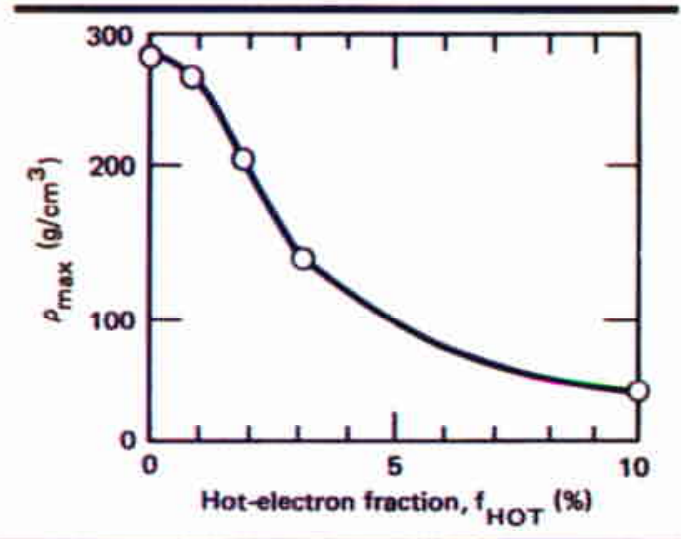

$(\sim 1000 \times)$, the fuel itself becomes a pusher. At the end of the implosion, the fuel is dense enough, cool enough, and moving fast enough to stagnate to very high densities; it gets little or no help from the pusher, so that the pusher and the fuel are essentially decoupled. Clearly, two-dimensional asymmetries will reduce the effectiveness of the fuel stagnating upon itself.

We now examine both parts of our hydrodynamic theory.

Role of the Fuel Adiabat. Our use of "adiabat" is explained with the aid of Fig. 2-15, which plots $\ln T$ vs $\ln \rho$ for several sections of cryogenic fuel. We define adiabat by extrapolating a line of slope $=$ 0.67 from the ideal portion of the $\ln T$ vs ln $\rho$ curve back to the $\rho=0.1 \mathrm{~g} / \mathrm{cm}^{3}$ axis. The temperature value of the intersect is our adiabat. [If some other reference density is preferred, then multiply our adiabat by $(\rho / 0.01)^{0.67}$ ]

Examining many one- and twodimensional implosion simulations leads us to the conclusion that the fuel naturally divides itself into three regions: the outer region: the inner region; and the central region, which lies between the outer and inner regions. Each region has its own dominant preheat mechanism, has a different adiabat, and reaches a different density. The three regions are shown in Fig. 2-16.

Referring to this figure, we see that the outer region of the fuel is preheated by thermal conduction from the pusher. With the nominal Shiva hohlraum model, the temperature of the pusher itself rises to about $5 \mathrm{eV}$ because of preheat by $x$ rays and hot electrons. (See Figs. 2-17 and 2-18 and Table 2-1 for a discussion of our model technique.)

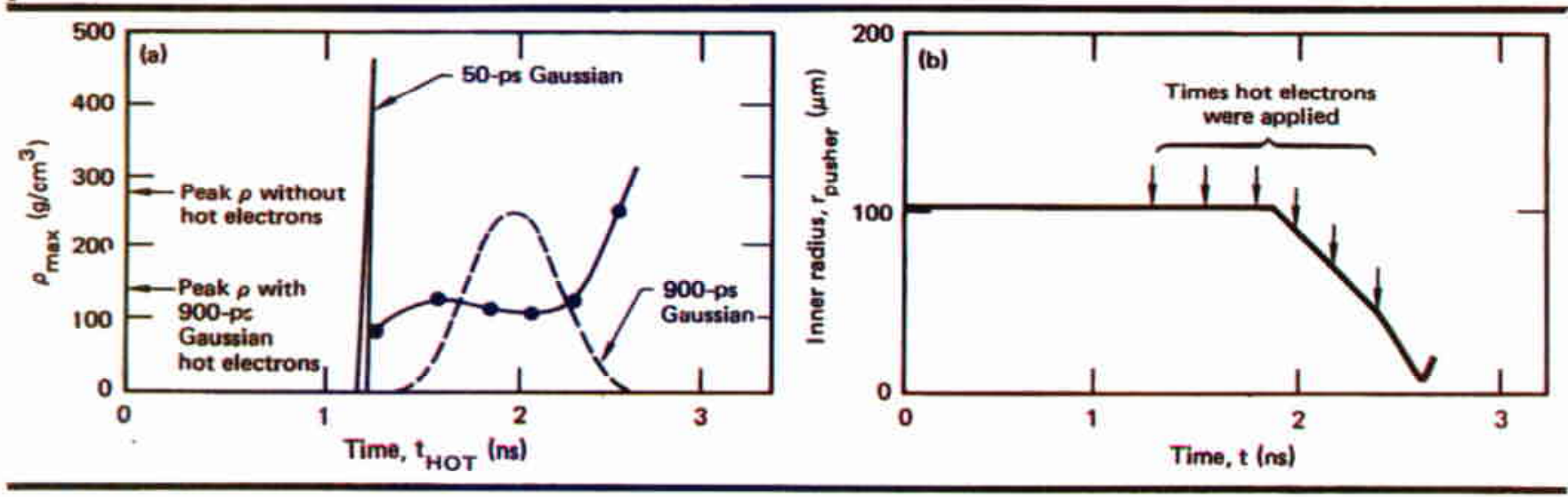


Heat flow from the pusher to the outer part of the fuel raises the adiabat of the outer-fuel mass to $\sim 2 \mathrm{eV}$.

The inner region of the fuel is dominated by shock preheat. When the pusher jumps-off, the fuel in this region is spalled. Late in the implosion, the accelerating pusher/fuel mass catches up to this expanded fuel and shocks it. Typically, the inner region of fuel is on a $10-$ to $20-\mathrm{eV}$ adiabat; however, this part of the fuel is very sensitive to the details of the velocity profile. Different target/hohlraum designs can place the inner-fuel mass on greatly differing adiabats.

The third region of the fuel is the central region, which is preheated by shock, by hot electrons, and by thermal conduction from the two hotter regions that surround it. Without hot electrons, the central fuel mass gets shocked-up to about $2 \mathrm{eV}$. Because this temperature is not high enough to fully ionize solid D-T, the central fuel mass lies on a very low adiabat, equivalent to an ideal gas at approximately $0.1 \mathrm{eV}$. The presence of hot electrons will raise the adiabat of the central fuel mass, as will thermal conduction. For our nominal Shiva model, the order of importance of preheat processes in the central-fuel region are hot electrons shock $>$ thermal conduction. For our Novette model $\left(\mathrm{f}_{\text {HOT }}<1 \%\right.$ ), the order of importance becomes shock $>$ thermal conduction $>$ hot electrons.
Figure 2-19 indicates how these three regions perform during an implosion. Density is plotted vs time for two one-dimensional implosions. One implosion has $3 \% \mathrm{f}_{\mathrm{HOT}}$. while the other has no hot-electron fraction. Qualitatively, both simulations behave the same, although the numerical values appearing are nearly a factor of two apart. As the sketch accompanying Fig. 2-19 shows, the central-fuel region attains the highest

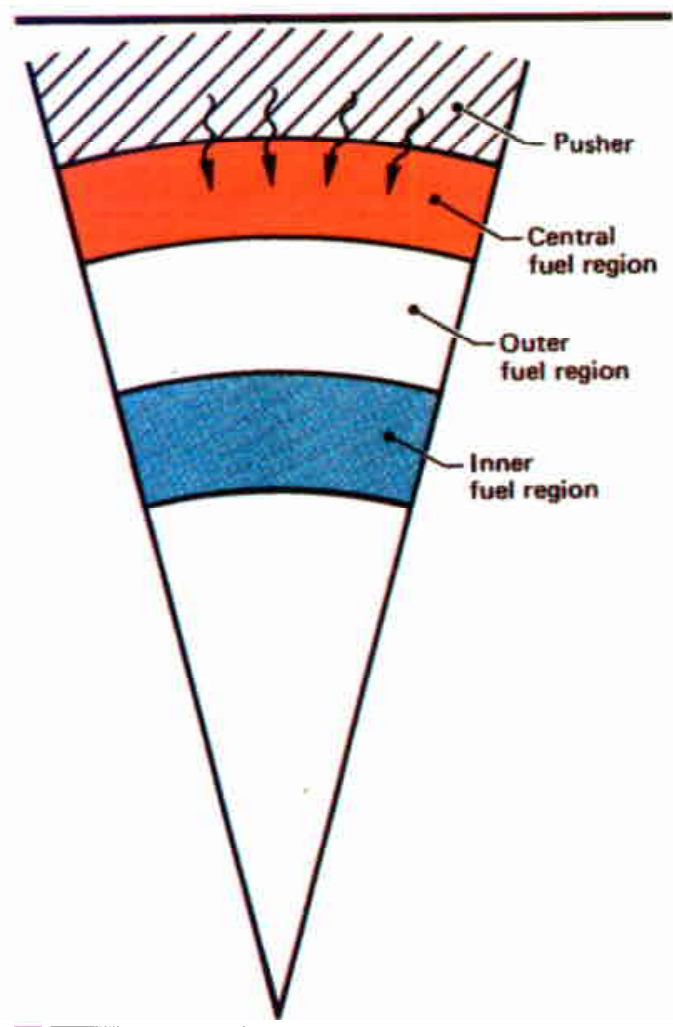

Fig. 2-15. The three characteristic regions of cryogenic fuel.
Fig. 2-16. Plots of In $T$ vs in $\rho$ for the implosions shown in Fig. 2-16. (a) With $3 \%$ hot-electron fraction. (b) Without hot electrons.

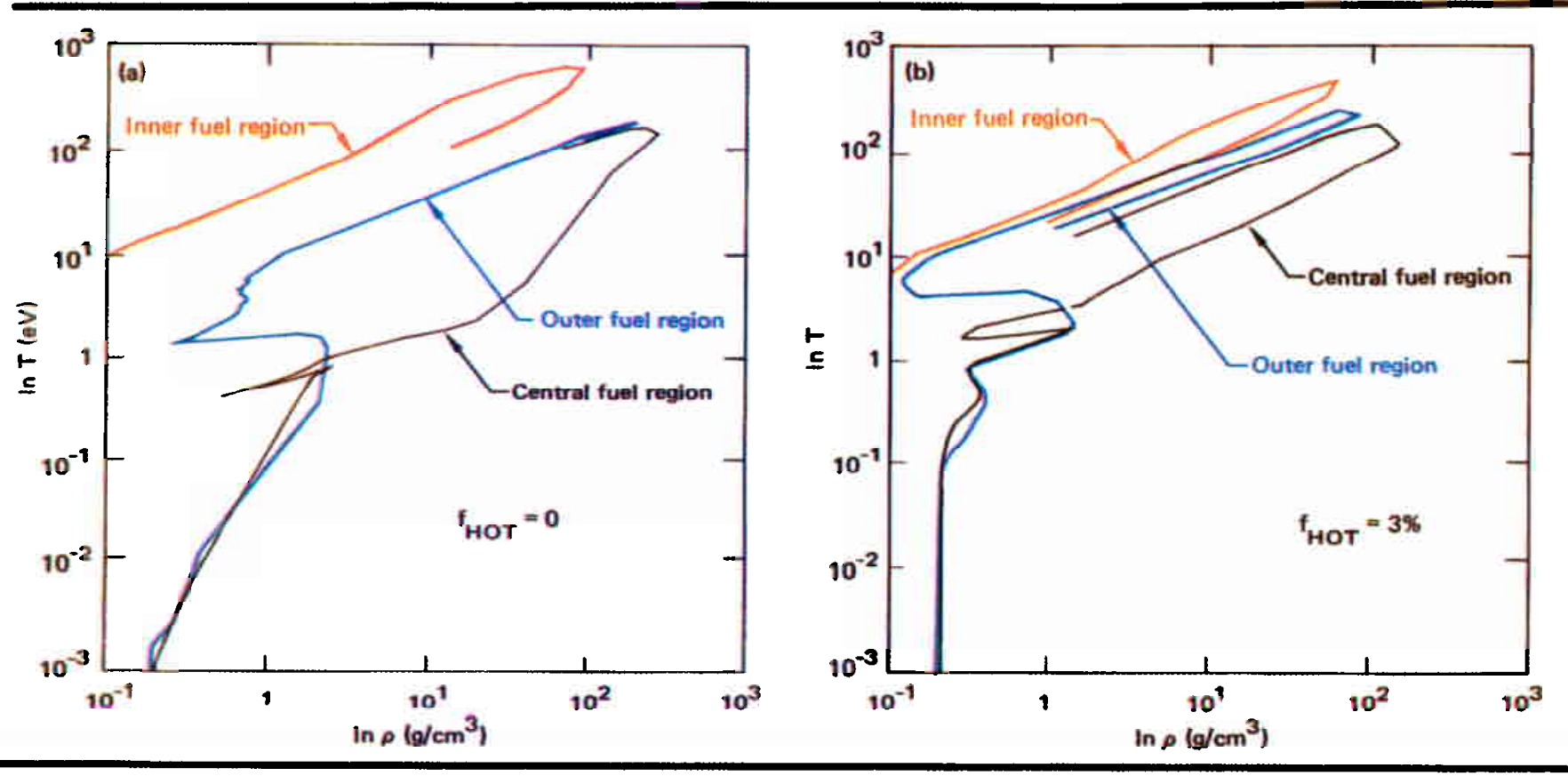


density, while the inner-fuel region attains the lowest density.

Figure 2-15 shows plots of $\ln T$ vs $\ln \rho$ for these same two implosions. These plots clearly show that the most dense region of the fuel is on the lowest adiabat, while the least-compressed (inner fuel) mass lies on the highest adiabat. We also note in

Fig. 2-15 that the two fuel adiabats associated with the most dense parts of the two implosions are significantly different. In the absence of hot electrons, as we have said, the central-region fuel mass initially appears to be partially ionized, and it compresses with a $\gamma<1.67$. However, with hot electrons present, this fuel mass appears to be fully ionized and implodes like an ideal gas

Table 2-1. Radiation in the drive spectrum $(\sim 0.4 \mathrm{keV})$ and the gold-line preheat spectrum ( $2.4 \mathrm{keV})$ for different hohlraum models.

\begin{tabular}{lccc}
\hline \multicolumn{1}{c}{ Model } & $0,4 \mathrm{keV}$ & $2.4 \mathrm{keV}$ & $3.0 \mathrm{keV}$ \\
\hline Nominal LASNEX & $7 \times 10^{-6}$ & $7 \times 10^{-8}$ & $1 \times 10^{-8}$ \\
High $\times$-ray preheat & $5 \times 10^{-6}$ & $5 \times 10^{-7}$ & $2 \times 10^{-7}$ \\
$\mathrm{~T}_{\text {rad source }}$ & $6 \times 10^{-6}$ & $5 \times 10^{-10}$ & $2 \times 10^{-11}$ \\
\hline
\end{tabular}

Note: table entries are in jerk/ $/ \mathrm{cm}^{3} \mathrm{keV}$, the nominal hohlraum is $6.7 \mathrm{~kJ}$ at 900 ps into a $5 / 8$-scale 4.0 Caim, $t=2.0$ ns, and $T_{R}=" 140 \mathrm{eV}$ "

Fig. 2-17. Hohlraum model using the technique of Pan. (a) Initial calculation. (b) Subsequent calculations.

Fig. 2-18. A 600-ps drive source for 5/8-scale 4.0 Caims target. (a) Comparison of Dante $\mathbf{T}_{\mathrm{rad}}$ vs $\mathrm{t}$ with our model. (b) Comparison of timeintegrated Dante spectrum with our model. (c) Comparison of experimental data and computational results using our model. $\mathbf{\nabla}$
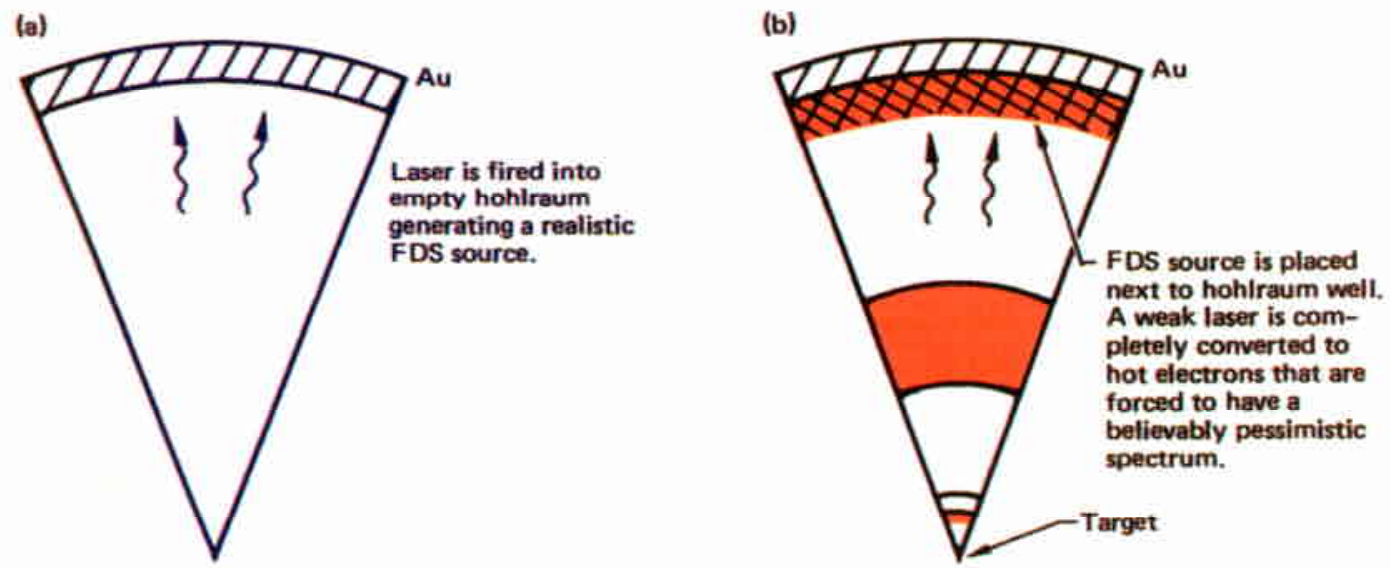

The sketch shows how we model one-dimensional implosions, illustrates a variation of the technique used by Y. Pan: Radiation preheat and drive come from an FDS source that we place naxt to the hohlraum wall. This FDS source is a "140-eV" source generated by separate empty-hohlraum laser calculations using $8 \mathrm{~kJ}$ in 900 ps.
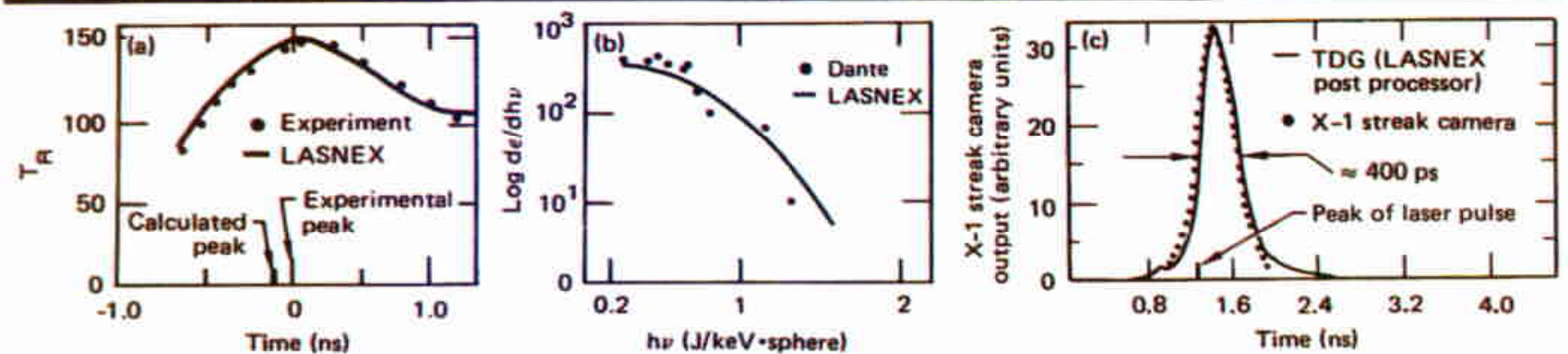

Our modeling technique produces radiation outputs that agree with experiments done on $5 / 8$-scale 4.0 Cairn targets irradiated by a 600 -ps pulse. The good agreement with fairly sketchy data is a healthy sign. Besides the "good" source, which we think models Shiva, we also have some other sources, notably a high $x$-ray preheat source that has about 10 times more preheat energy than our nominal model. Although we do not believe this source is accurate, it is useful for evaluating worst-case scenarios and for bringing out physics points. Table 2-1 compares preheat and drive for various radiation models. Hot electrons are included in the model by shining an appropriate amount of laser energy onto the hohlraum wall and converting the energy entirely into 50 -keV hot electrons. 

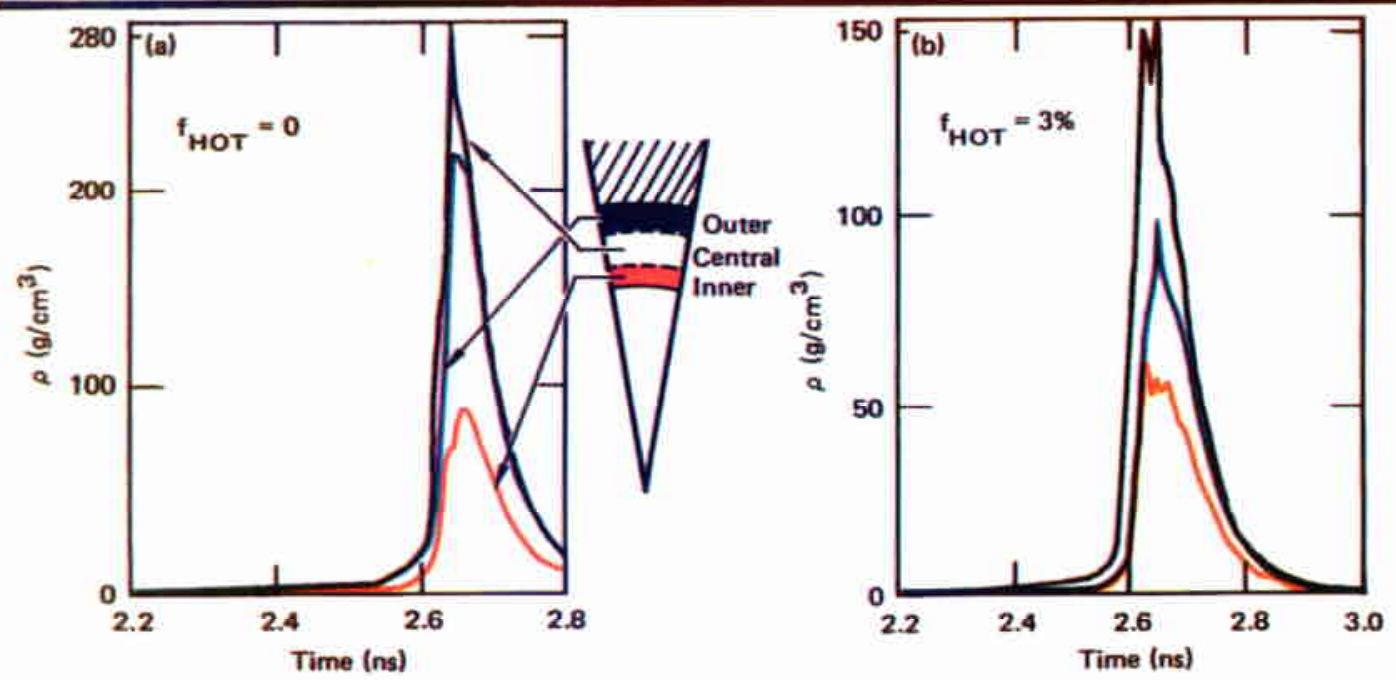

processes that will raise the adiabat of the central fuel mass: thermal conduction and shock heating. For simulations without the presence of hot electrons, Fig. 2-21 shows peak density of the central-fuel mass as a function of a thermal conduction multiplier. These calculations were done using Cray LASNEX, which is thought to provide a better treatment of heat transport than the 7600 version. ${ }^{4}$ We can see from the graph that inaccuracies in our understanding of thermal conduction could be important. Ten times more conductivity than we think is correct would substantially degrade the capsule's performance, and one-tenth the transport would essentially eliminate all the effects of heat flow.

Shock heating also raises the adiabat of the central-region fuel mass. Keeping shock heating down is the reason we would like to implode these capsules with a 900-ps pulse, rather than with the more usual 600-ps pulse. Moreover, in optimizing Shiva implosions for which hot electrons are present, we have also found it necessary to reduce the thickness of $\mathrm{CH}$ below that called for by the rocket equation; a "proper" thickness of $\mathrm{CH}$ results in a too-hard shock hitting slightly expanded fuel, putting the central-fuel mass on too high an adiabat.

Shock heating can also be reduced in other ways, all of which seem to be equivalent to pulse shaping. For example, one of the observations we mentioned earlier was that calculations done with very high $x$-ray preheat show achievement of extremely high density and pressure. Part of the reason for this is that the hard $x$ rays cause the glass to swell. This swelling then com-
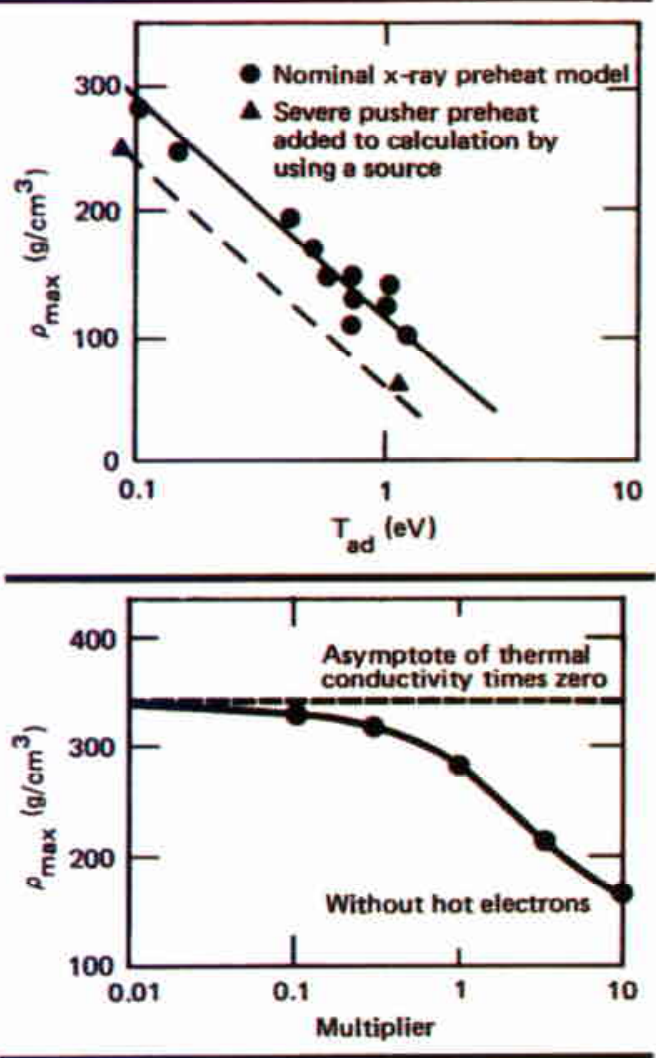

presses, accelerates, and shocks the cryogenic layer to about $0.2 \mathrm{eV}$. Figure 2-22 compares fuel density vs time $(\rho-t)$ and temperature vs time $(\mathrm{T}-\mathrm{t})$ at jump-off for the high $x$-ray preheat model and for the normal model without hot electrons present. When the main shock hits in the high $x$-ray preheat model, the density jump and temperature jump of the fuel correspond to a lower adiabat than for the normal x-ray model. The main shock leaves the fuel on a lower adiabat because of early motion-which is the same thing as pulse shaping.
Fig. 2-19. Density vs time plots for implosions. (a) Without $3 \%$ hot-electron fraction. (b) With 3\% hotelectron fraction.

Fig. 2-20. Maximum fuel density vs adiabat of the piece of fuel that hit peak density. Different calculations led to different adiabats because of variations in the hohlraum model.

Fig. 2-21. Maximum fuel density vs LASNEX thermal conduction multiplier. A thermal transport greater than Cray LASNEX predictions could raise the adiabat of the central-fuel region and cut the peak density. 
Fig. 2-22. Swelling of the pusher due to large amounts of $x$-ray preheat. (a) Low $x$-ray preheat. (b) High $x$-ray preheat.

Laser Targets

Pusher-Fuel Decoupling. One of our puzzling observations about these targets is that the peak fuel pressure can greatly exceed the peak stagnation pressure of the same hydrodynamic system imploded without fuel. Table 2-2 shows peak pressures with and without fuel for various hohlraum models. In some models, the peak pressures with and without fuel are roughly comparable. However, in other systems with cold fuel but preheated pushers, the peak pressure of the fuel can be far greater than that generated by the hydrodynamic system itself.

The physical reason for this is that, to achieve the highest densities with glass and $\mathrm{CH}$ cryogenic targets, it is not necessary for the pusher to stagnate upon the fuel. If the fuel is on a low enough adiabat, then even a severely preheated pusher can drive the fuel fast enough so that it forms a cold, dense, rapidly imploding shell. When this shell stagnates, it is capable of generating the peak conditions we calculate-without outside help from the pusher.

To see this, let us focus on the calculation in Table 2-2 labeled "Pusher preheat added as source." Figure 2-23 illustrates the fuel configuration of this run at $2.6 \mathrm{~ns}$ - just before stagnation. We see that the fuel itself is a cold $(<10 \mathrm{eV})$, dense $\left(\approx 30 \mathrm{~g} / \mathrm{cm}^{3}\right)$, rapidly moving shell. Looking at the energy of the $L=4$ zone, we see that the sum of the internal energy and kinetic energy is $\mathbf{0 . 0 0 2}$ jerk $/ \mathrm{g}+0.018 \mathrm{jerk} / \mathrm{g}=0.020$ jerk $/ \mathrm{g}$ at $\mathrm{t}=$ $2.6 \mathrm{~ns}$. When it stagnates, this chunk of fuel comes up to $450 \mathrm{~g} / \mathrm{cm}^{3}$ and $98 \mathrm{eV}$. At this $\rho$ and $T$, the energy density of the fuel is 0.021 jerk $/ \mathrm{g}$. This piece of fuel can achieve its peak conditions merely by stagnating upon itself; almost no energy need be transferred to it. In examining the $\mathrm{L}=14$ part of the fuel, we see that this cold, dense, rapidly moving fuel mass has an energy density of 0.016 jerk $/ \mathrm{g}$ just before stagnation. At its peak condition, the energy density

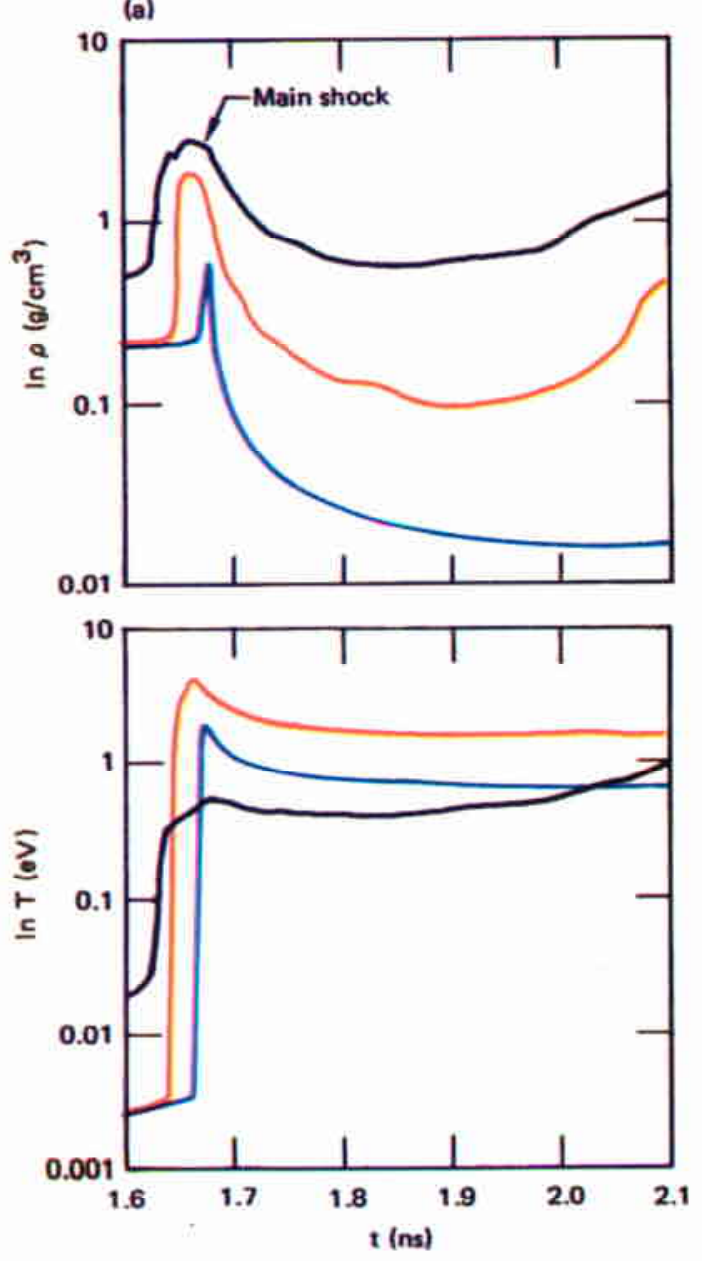

(b)
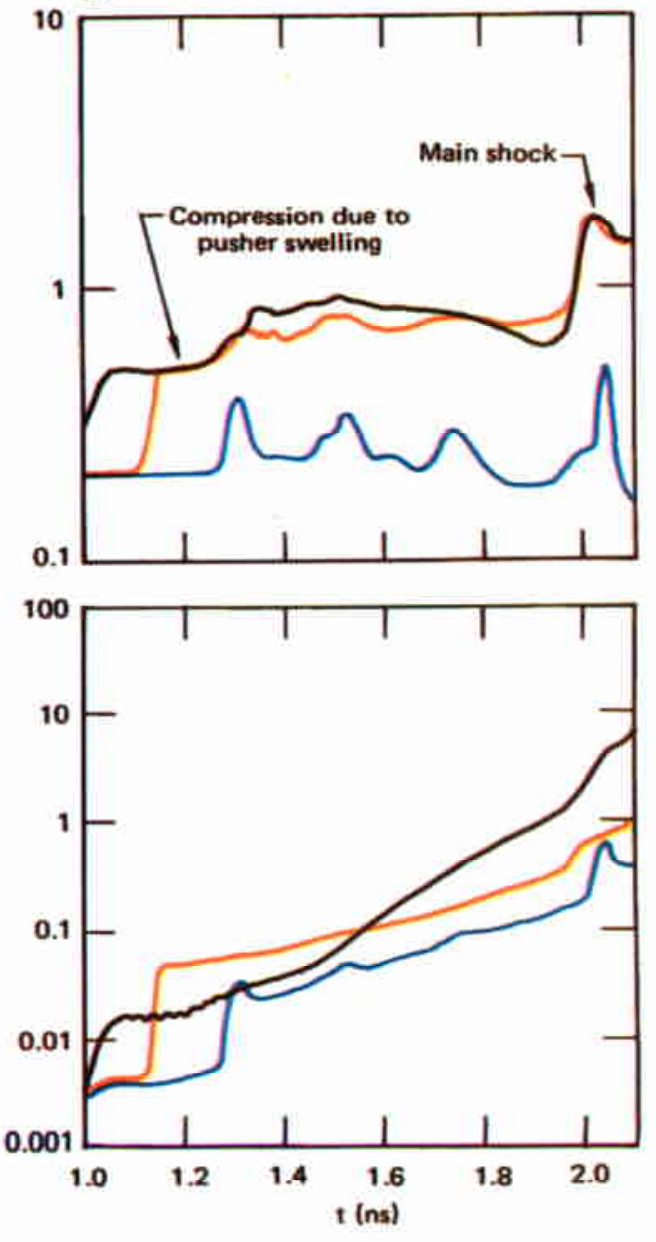
rises to $0.020 \mathrm{jerk} / \mathrm{g}$, so there is only a small transfer of energy to this section of fuel.

Only the outermost, lowest-density part of the fuel is substantially influenced by the pusher. During the period from $2.6 \mathrm{~ns}$ to peak density, the energy density of this part of the fuel nearly doubles, from 0.014 to $0.023 \mathrm{jerk} / \mathrm{g}$.

Apparently, these targets do not have to convert pusher kinetic energy into fuel internal energy to achieve high density. For example, in high-pusher-preheat implosions, the total fuel energy rises only $40 \%$ from the time the pusher starts decelerating until it is fully stagnated. Analysis of the numbers shows that most of this energy goes into the lower-density regions nearest the pusher; the highest-density fuel gains very little energy. In low-pusher-preheat implosions, where we would expect better transfer of pusher energy, the total fuel enengy doubles during stagnation. Again, however, nearly all of this energy appears in the lower-density regions.

Interestingly enough, the specific energy of the most dense part of the fuel is fairly independent of peak density. This is seen in Fig. 2-24, which plots specific energy of the maximum-density fuel vs $\rho_{\max }$ These points were taken from a variety of calculations in which we varied the maximum density by varying the preheat; drive and capsule design were kept fixed. Apparently, the energy of the highest-density fuel is determined by its peak kinetic energy-a quantity that remains fairly constant as preheat is varied.

LASNEX seems to be telling us that stagnation of the pusher is not a particularly important part of these implosions.

Implications of Self-Stagnation. What does all this mean? First of all, we do not need dense metal next to the fuel to attain $1000 \times$. Glass pushers, even those expanded by preheat, can still drive D-T fuel up to a very high density. The fuel itself can become a cold, dense, fast-moving shell.

Second, the fuel-pusher decoupling implies that the state of the fuel may not be reflected by the state of the pusher. Specifically, this means that neutron average $\rho \Delta r$ of the pusher may not be a unique diagnostic of the state of the fuel. Figure 2-25, which plots peak fuel density vs pusher $\rho \Delta \mathrm{r}$, justifies this statement. A low $\rho \Delta \mathrm{r}$ of the pusher does not necessarily indicate low fuel density. To infer a fuel density, we need to know something about the hohlraum conditions. The latter point is emphasized by the dashed lines in the figure, which represent calculations where the pusher (but not the fuel) was preheated far more than our nominal model would predict.

By contrast, the points of density vs $\rho \Delta \mathrm{r}$ for room-temperature gas-filled capsules (Fig. 2-26) do seem to lie on one line that is independent of the hohlraum model. Here, pusher stagnation is required to achieve peak density, and $\rho_{\max }$ is a simple function of pusher $p \Delta \mathbf{r}$. (Also indicated in the figure is the uncertainty expected in a radiochemical measurement.) By making our targets cryogenic, we have introduced ambiguity into the interpretation of the $\rho \Delta \mathrm{r}$ results. Fortunately, the NPIRE I and NPIRE II series will provide us with data that will allow us to form a relatively good idea of the drive and preheat of our hohlraum.

\begin{tabular}{|c|c|c|}
\hline Model & $\begin{array}{l}\text { Peak p } \\
\text { Without foel }\end{array}$ & $\frac{\left.\mathrm{k} / \mathrm{cm}^{3}\right)}{\text { With fijel }}$ \\
\hline Diffusion $f_{H O T}=3 \%$ & 22 & 24 \\
\hline Diffusion & 5.0 & 5.2 \\
\hline High $x$-ray preheat & 052 & 30 \\
\hline Pusher preheat added as source & 26 & 80 \\
\hline PBINIR without bot electrons & 21 & 27 \\
\hline PBINTR with $3 \%$ hot electrons & 0.9 & 0.9. \\
\hline
\end{tabular}
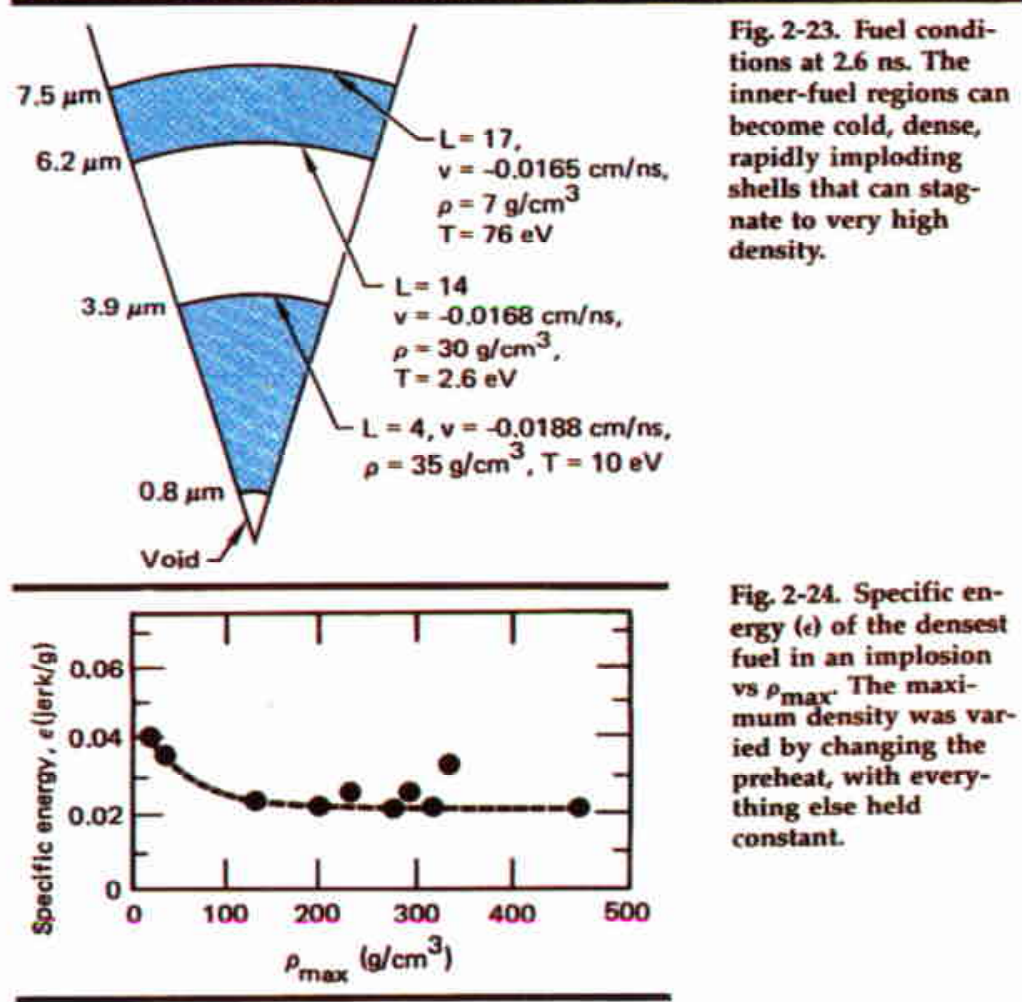

Fig. 2-24. Specific energy (e) of the densest fuel in an implosion vs $P_{\max }$ The maximum density was varied by changing the preheat, with everything else held constant. 
A third implication of pusher-fuel decoupling is that mix may not be a major problem in these targets. The reason is that peak density does not occur because the pusher squeezes the fuel as it slows down; peak density occurs due to incoming fuel stagnating on dense inner fuel. A spherical shock of high-density, high-pressure fuel grows outward; when the shock hits the pusher and decelerates it, the outer fuel is actually denser than the pusher. At peak fuel density, the pusher density at the fuel-pusher interface usually is only slightly greater than the fuel density, indicating that growth and penetration of Rayleigh-Taylor mix should not be a major factor in these capsules.

Independence of Hot-Electron Timing. One of our observations about these targets is that their performance seems to be rela-

Fig, 2-25. Peak fuel density vs neutronaveraged pusher $\rho \Delta \mathbf{r}$ for cryogenic targets. tively independent of hot-electron timing. This is shown in Fig. 2-14, which plots $\rho_{\max }$ vs $t_{\text {HOT }}$, where $t_{\text {HOT }}$ is the time at which we

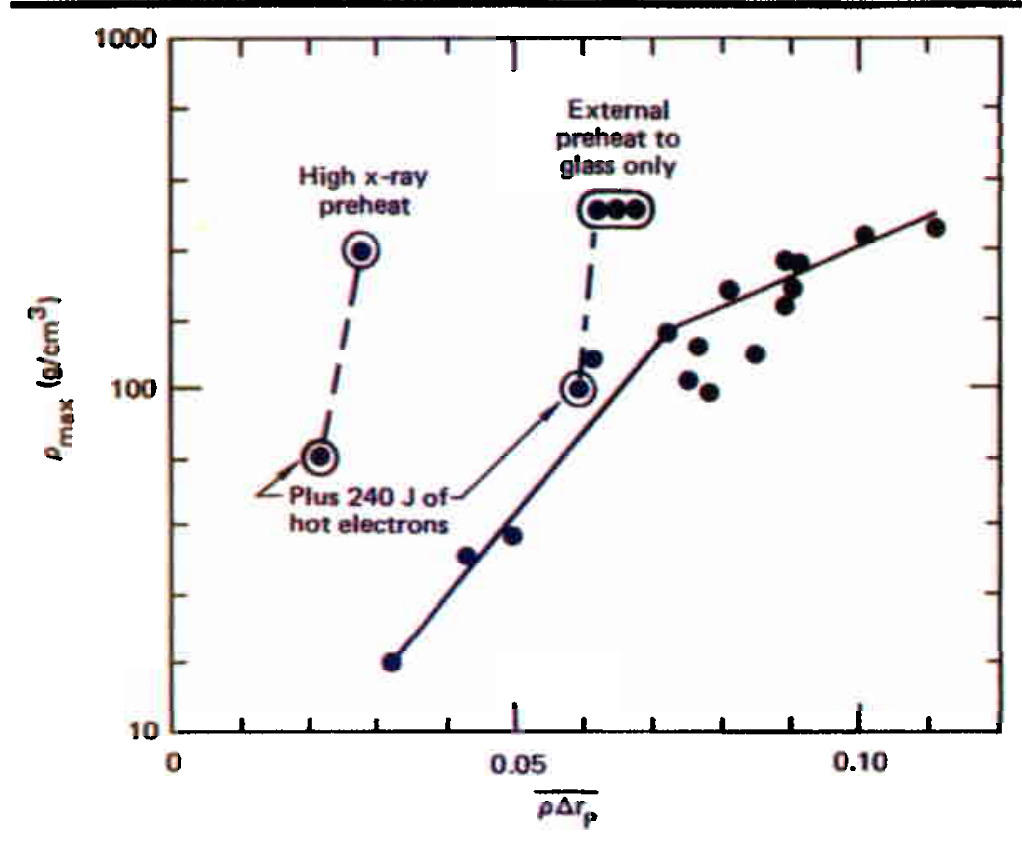

Fig 2-26. Peak fuel density vs pusher $p \Delta r$ when the targets are gas filled instead of cryogenic.

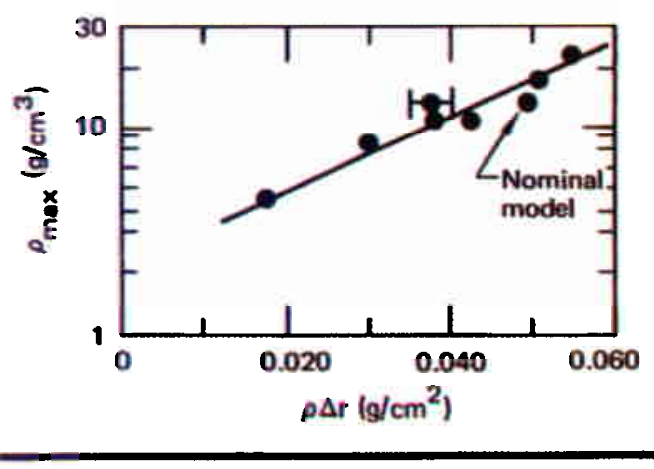

applied $3 \% \mathrm{f}_{\mathrm{HOT}}$ with a 50-ps FWHM.

Figure 2-14 shows us that we get about the same $\rho_{\max }$ if the hot electrons appear almost. anytime during the main part of our laser pulse. Only at very early or very late times will there be a difference. (Apparently, at late times, the pusher becomes thick enough to shield the fuel from the hot electrons. At early times, the fuel has an opportunity to expand and behave more like a gas-filled implosion.)

The physical reason why hot-electron timing is not important seems to be that the fuel ends up at nearly the same adiabat regardless of when the preheat appears. LASNEX indicates that a pulse with a $3 \%$ hot-electron fraction followed by a shock causes roughly the same total preheating as a shock followed by the hot electrons. We see this in Fig. 2-27, where we plot fuel adiabat vs time of appearance of the hotelectron pulse. We argue that our targets are relatively independent of hot-electron timing because the fuel adiabat is independent of timing and because it is the adiabat of the fuel that determines the peak density.

Neutron Yield. Figure 2-28 shows neutron yield vs $f_{H o r}$ for one-dimensional implosions using diffusion transport. The yield varies by only a factor of 3 as we change $\mathrm{f}_{\mathrm{HOT}}$ from 0 to $10 \%$. Examination of these calculations indicates that $90 \%$ of the yield comes from the inner one-eighth of the fuel mass, which is the fuel that is first spalled and then shocked to a high adiabat. At peak compression, the outer radius of the neutron-producing region is more than one-half the total fuel radius.

A traditional way to cut our expected neutron yield is to dud the center part of the fuel. The usual argument for dudding the center is, "The central neutrons originate from a shock stagnating at the origin. In a real three-dimensional target, this will not happen." However, we have performed many two-dimensional calculations to examine the effect of asymmetric implosions on our capsule. As noted below, we have found, very reproducibly, that the yields do not appear to change greatly due to two-dimensional effects. So, rather than clinging to bias, we claim that these calculated yields are the ones we would expect-if the hohlraum implodes the capsule in a manner indicated by a two-dimensional diffusion model. 
While symmetry and hot electrons do not appear to have a great effect on the yield, variations in the radiation model do change the yield. Table 2-3 lists yields and densities for several models. The first four cases would be characterized as " $140-\mathrm{eV}$ sources"; nevertheless, each case produces a different drive and preheat.

If real Shiva or Novette hohlraums behave like some of these other radiation models, it would appear that we may not have enough yield to do a diagnoseable experiment. Fortunately, there is at least one way we can trade density for yield in these targets: we can fill the void in the center of our cryogenic target (see Fig. 2-12) with low-density "boost gas." This boost gas raises the PBINTR yield of the target from $\sim 1 \times 10^{6}$ to $5 \times 10^{7}$ while cutting the fuel density from $\sim 350$ to $200 \times$.

Nature appears to have provided an enticing near-term source of boost gas, one that could even be used on Shiva. It seems that, over a several-degree temperature range near the D-T triple point, liquid D-T will spread itself into a continuous, but nonuniform, layer over the inside of a glass microballoon. ${ }^{5}$ At around this temperature, the vapor pressure (see Fig. 2-29) corresponds to a density of about $0.001 \mathrm{~g} / \mathrm{cm}^{3}$. Moreover, two-dimensional calculations indicate that some sagging of this layer (up to $30 \% \mathrm{P}_{1}$ defect) should not greatly affect either the yield or peak density. (Note: the term $P_{1}$ measures the distance between the center of the outside of a shell and the center of the inside of a shell.)

Author: L.J. Suter energy density by a multiplier that was 1.0 at the waist and pole and $1.0+\mathrm{f}$ at $\theta^{\circ}$ $=135^{\circ}$ (i.e., the $45^{\circ}$ point). The term $\mathrm{f}$ is the parameter we varied in this survey; it tells us how much energy asymmetry we have in the hohlraum.

Figure 2-31(a) shows how peak density of our targets drops with asymmetry. According to the figure, we can have up to $20 \%$
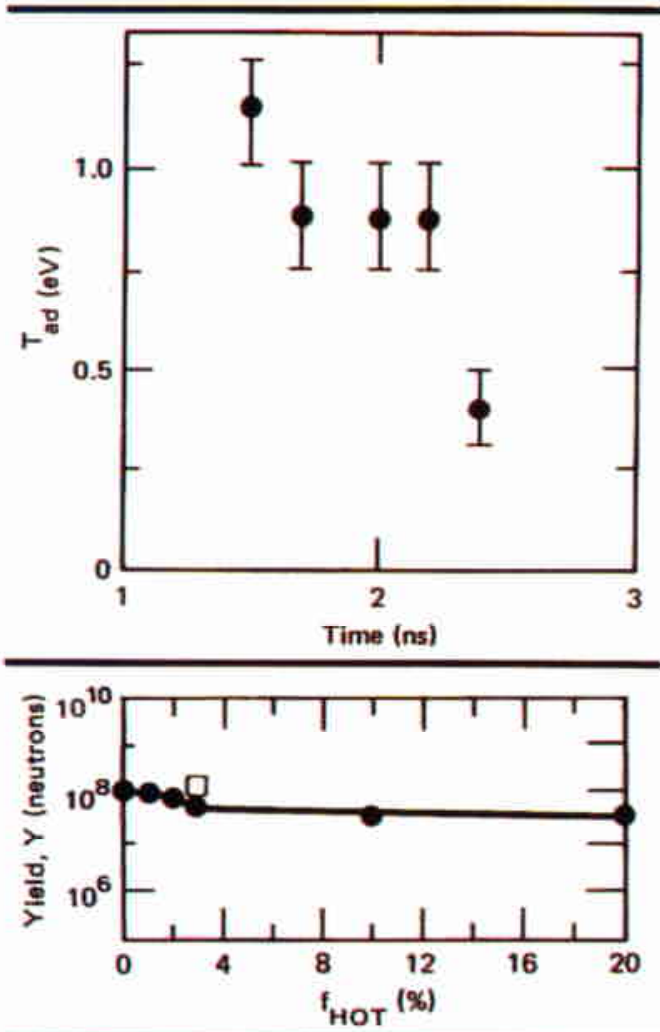

Fig. 2-28. Neutron yield as a function of $f_{H O T}$ The yield is fairly insensitive to $f_{\text {HOT }}$ on a nominal target. The square in the figure is for very finely zoned fuel.

\begin{tabular}{|c|c|c|}
\hline Model & With hot electrons & Without hot electrons \\
\hline $\begin{array}{l}\text { Nominal radiation with } \\
\text { diffusion transport and } \\
3 \% \text { hot electrons }\end{array}$ & $150 \mathrm{~g} / \mathrm{cm}^{3}, 1 \times 10^{8}$ & $280 \mathrm{~g} / \mathrm{cm}^{3}, 1 \times 10^{8}$ \\
\hline $\begin{array}{l}\text { High } x \text {-ray preheat } \\
\text { and } 3 \% \text { hot electrons }\end{array}$ & $30 \mathrm{~g} / \mathrm{cm}^{3}, 1 \times 10^{5}$ & $250 \mathrm{~g} / \mathrm{cm}^{3}, 1 \times 10^{5}$ \\
\hline Only pusher preheated & - & $380 \mathrm{~g}^{-\mathrm{cm}^{3} .1 \times 10^{5}}$ \\
\hline $\begin{array}{l}\text { Nominal radiation with } \\
\text { PBiNTR and } 3 \% \text { hot } \\
\text { electrons }\end{array}$ & $80 \mathrm{~g} / \mathrm{cm}^{3}, 1 \times 10^{6}$ & $230 \mathrm{~g} / \mathrm{cm}^{3} .2 \times 10^{6}$ \\
\hline $\begin{array}{l}\text { Nominal radiation with } \\
\text { PBINTR plus boost gas } \\
\text { and } 3 \% \text { hot electrons }\end{array}$ & $40 \mathrm{~g} / \mathrm{cm}^{3}, 3 \times 10^{7}$ & - \\
\hline $\begin{array}{l}\text { Novette, } 160 \mathrm{eV} \\
\text { PBINTR with } 1 \% \text { hot } \\
\text { electrons }\end{array}$ & $300 \mathrm{~g} / \mathrm{cm}^{3}, 5 \times 10^{7}$ & - \\
\hline $\begin{array}{l}\text { Novette, } 160 \mathrm{eV} \text {, } \\
\text { PBINTR with } 1 \% \text { hot } \\
\text { electrons, boost gas }\end{array}$ & $130 \mathrm{~g} / \mathrm{cm}^{3}, 1 \times 10^{9}$ & - \\
\hline
\end{tabular}

Table 2-3, Onedimensional peak target densities and neutron yields for various hohlraum models. 
Fig. 2-29. Vapor pressure-temperature curves for hydrogen, including D-T. (Redrawn from Roder

et al., Survey of the

Properties of the $\mathrm{Hy}$ -

drogen Isotopes Belotw

Their Critical Tem-

peratures, National

Bureau of Standards,

Boulder, Colo., Tech-

nical Note 641 (1973),

p. 83.)

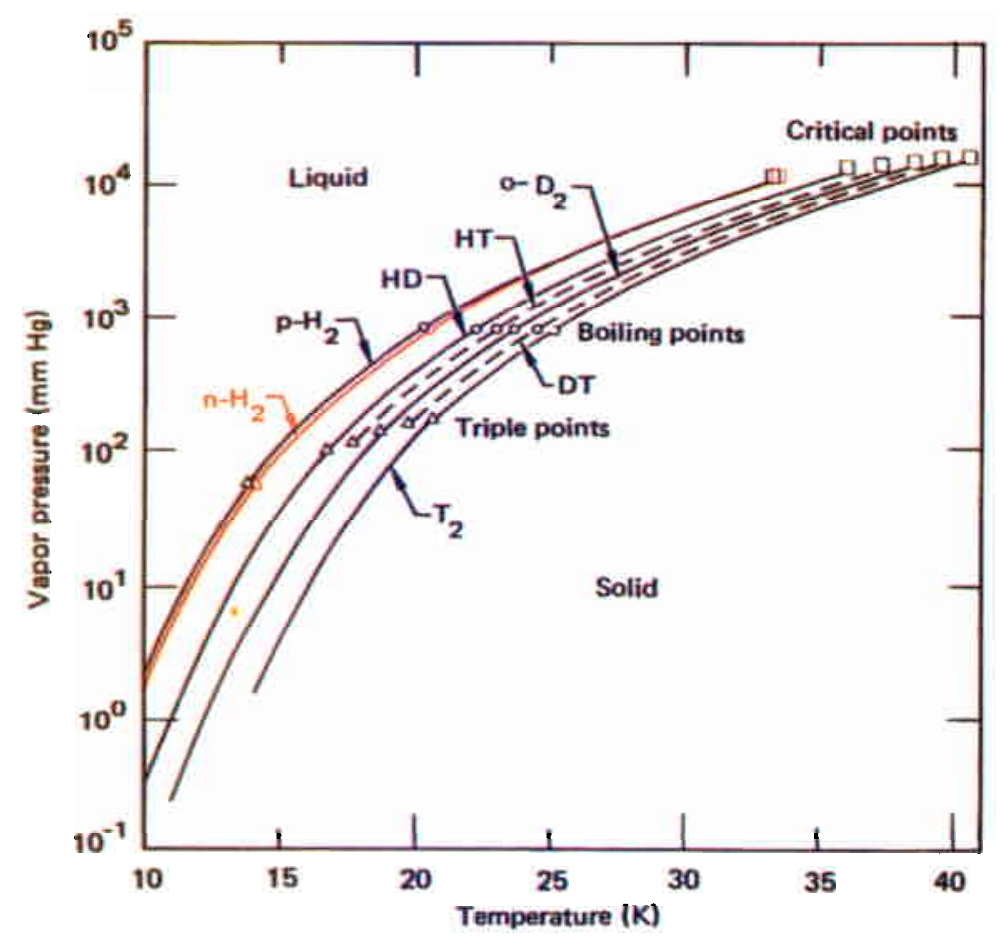




\section{Apollo High-Density Targets}

The success of the first Shiva-scale, double-shell, intermediate-density target (Fig. 2-34), code named Apollo, demonstrates that the LASNEX computer program can be used to correctly predict the performance of complex targets and gives us 


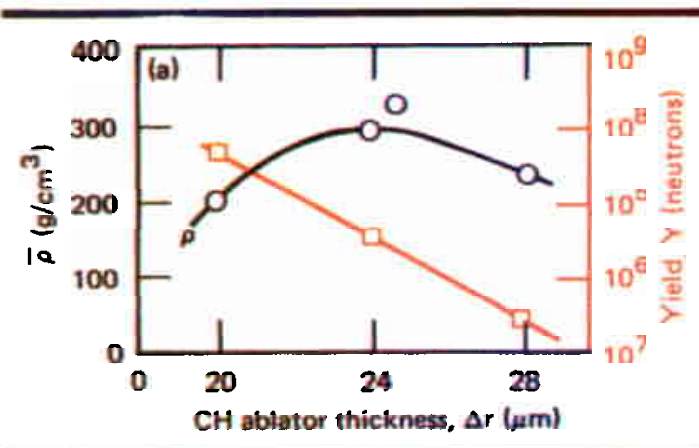

added incentive and confidence to pursue and explore the merits of more advanced Apollo target designs. Since the preshot Apollo design study results and the preliminary postshot analysis of the experimental data have been presented elsewhere (Ref. 7 and "Apollo Intermediate-Density TargetsAnalysis" in Section 4 of this report), we discuss here the near-term potential of the glass-tamper double-shell targets and describe more advanced high-density Apollo targets that will be driven by $x$ rays generated by the absorption of 0.25 - to $0.50-\mu \mathrm{m}$-wavelength laser radiation.

Glass-Tamper Targets. Glass-tamper Apollo targets similar in design to that shown in Fig. 2-34 can be used to experimentally examine several issues critical to the design of double-shell tangets (e.g., spherical convergence or symmetry requirements, shell sensitivity to hydrodynamic instabilities, deleterious effects of tamper-fuel mixing, shell coupling efficiency, and target-fabrication tolerances). However, these issues will not be addressed here; we will only indicate the near-term potential of obtaining fuel densities greater than $20 \mathrm{~g} / \mathrm{cm}^{3}$ ( $100 \times$ liquid density) by using glass-tamper targets.

One-dimensional LASNEX simulations of the Shiva-scale Apollo target showed that the high suprathermal-electron preheat of the glass tamper was the major cause of the relatively low compression achieved. Recent spherical hohiraum experiments have obtained a peak radiation temperature of $140 \mathrm{eV}$ and values of about 3 to $7 \%$ for $\mathrm{F}(\mathrm{HOT})$, which is the fraction of incident laser energy that is in suprathermal electrons (see "NPIRE" in Section 4). Such a large reduction (about $10 \times$ ) in the suprathermalelectron flux will clearly allow the fuel to be compressed to higher densities. As discussed previously, the peak fuel density achievable in an Apollo target is inversely

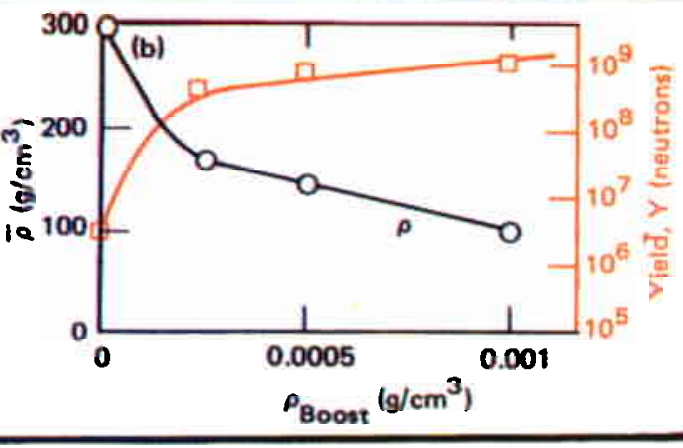

Fig. 2-33. (a) Peak average density and neutron yield vs ablator thickness for cryogenic 200- $\times$ 5- $\mu \mathrm{m}$ capaules imploded with $T_{R}-160 \mathrm{eV}$ and $f_{H O T}=1 \%$. (b) Effects of adding boost-gas to a cryogenic 200- $\times 5$ - $\mu \mathrm{m}+$ 24-mm-CH Novette target.
Fig. 2-34. Onedimensional diagram of the Shiva-scale Apollo intermediatedensity target. proportional to the initial tamper radius, but any increase in the fuel compression is obtained at the expense of lower neutron yield. Also, the smaller tamper radius forces the outer shell to undergo more spherical convergence.

Table 2-7 shows the one-dimensional LASNEX simulation results for two Apollo targets that have 120- $\mu \mathrm{m}-\mathrm{i}$.d. glass tampers of 10 - and $12.8-\mu \mathrm{m}$ thicknesses, are filled with D-T gas at $10 \mathrm{mg} / \mathrm{cm}^{3}$, and use $\mathrm{CH}$ outer shells as shown in Fig. 2-34. The LASNEX model used in these calculations has previously been described. ${ }^{7}$ A highpreheat frequency-dependent source (FDS) of $x$ rays with a peak radiation temperature of $120 \mathrm{eV}$ was imposed in the channel. (The time-integrated spectrum of this FDS source was identical to the "pessimistic" profile shown in Ref. 7.) Concurrently, a 1.4-kJ, $1.06-\mu \mathrm{m}$ laser beam was directed at the inside of the Au hohlraum; all of the incident laser energy was absorbed and placed into suprathermal electrons. The suprathermalelectron temperature, T(HOT), varied with the laser intensity according to the empirical formula in LASNEX and reached a peak value of $13 \mathrm{keV}$.

The results in Table $2-7$ indicate that peak fuel densities in excess of $40 \mathrm{~g} / \mathrm{cm}^{3}$ 
Fig. 2-35. (a) Onedimensional diagram of a 0.25 - to $0.50-\mu \mathrm{m}$ wavelength cryogenic D-T target. (b) Pulse shape of incident laser beam.

Table 2-7. Calculated results for two Shiva-scale glass-tamper Apollo targets.

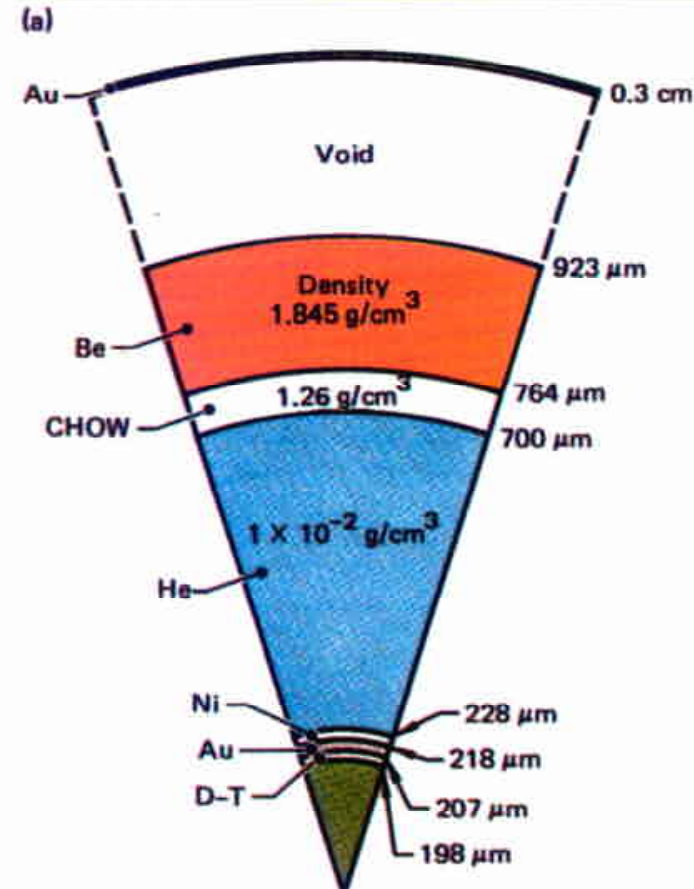

(b)

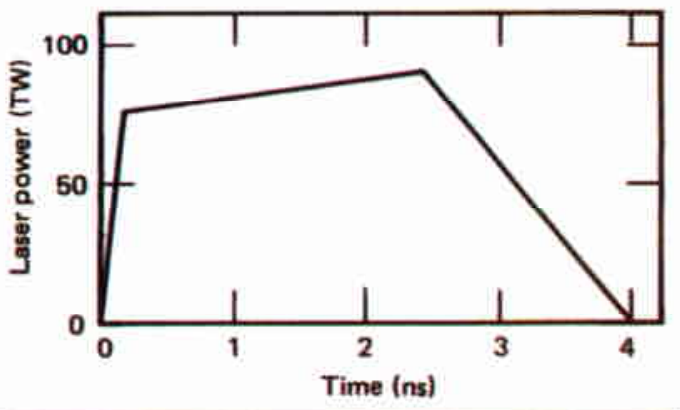

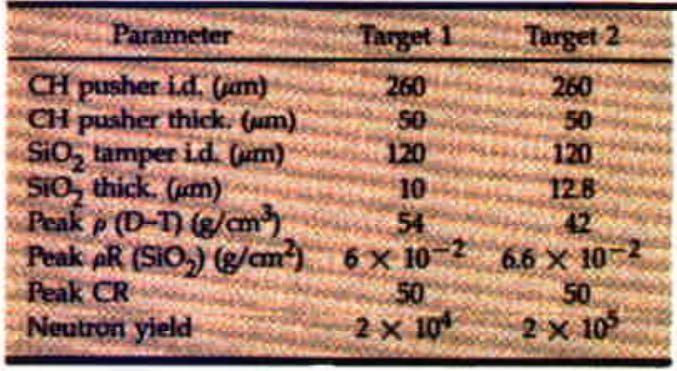

can be achieved in the near-term by making minor modifications to the previously tested Shiva-scale Apollo target and by using a spherical hohlraum. There is a moderate increase in the convergence-ratio requirement. (The convergence ratio, $C R$, is the initial outer radius of the ablator divided by the compressed-fuel radius.) We can increase the calculated low neutron yields by about an order of magnitude by using a higher peak $x$-ray hohlraum temperature $(140 \mathrm{eV})$. Nevertheless, it may be difficult to verify the compression achieved using the existing neutron-activation technique.

We have not fully explored the possibility of obtaining much higher fuel compression by using a glass-tamper Apollo target similar to that shown in Fig. 2-34 under conditions of low preheat and high $\mathrm{x}$-ray drive. A further increase in peak fuel density can be expected with either a smaller tamper radius, with a layered glass/high-Z tamper (e.g., glass/Pt), or both. However, limitations of existing diagnostics place a serious constraint on verifying the achievement of very high fuel compression. The development of new density-diagnostic techniques is clearly necessary.

0.25- to 0.50- $\mu \mathrm{m}$-Wavelength Apollo Targets. The use of $0.25-$ to $0.50-\mu \mathrm{m}$ laser radiation, which can be obtained either from a $\mathrm{KrF}$ laser or by frequency conversion of the 1.06- $\mu \mathrm{m} \mathrm{Nd:glass} \mathrm{laser} \mathrm{radiation,} \mathrm{is}$ expected to reduce the suprathermal electron production and to increase both the laser absorption and the $x$-ray conversion efficiency (ratio of radiated $x$-ray energy to laser energy) in hohlraum targets. Using one-dimensional LASNEX simulations, we have made a preliminary design study of double-shell targets with a single fuel region using 10 to $265 \mathrm{~kJ}$ of $0.33-\mu \mathrm{m}$ laser radiation (approximately midway in the 0.25 - to $0.50-\mu \mathrm{m}$ range). In this study, the basic components of the targets remained fixed, while the fuel mass (target size) varied according to the available laser energy. We present some unoptimized results of full-scale $(265-\mathrm{kJ})$ and 0.55 -scale $(50-\mathrm{kJ})$ targets to indicate the performance-parameter regime.

Throughout the one-dimensional LASNEX simulations, the laser radiation was initially directed at the Au hohlraum and was absorbed by both classical and nonclassical processes. The suprathermalelectron fraction, $\mathrm{F}(\mathrm{HOT})$, either was determined by the normal LASNEX parameters 
or was imposed. In some cases, the suprathermal-electron temperature, T(HOT), varied as a function of the laser intensity according to the empirical T(HOT) formula: in other cases, the parameters in the $\mathrm{T}(\mathrm{HOT})$ formula were adjusted to give a desired maximum value for $\mathrm{T}(\mathrm{HOT})$. Because of the relatively low peak laser intensities (about $1 \times 10^{14} \mathrm{~W} / \mathrm{cm}^{2}$ ) used in these tanget designs, we do not expect copious production of suprathermal electrons. When the normal LASNEX parameters were used, all the laser energy was absorbed by inverse bremsstrahlung (IB) before reaching the critical-density surface. When F(HOT) and T(HOT) were imposed, all the laser energy was forced to be absorbed at critical density with the specified energyabsorption fractions (IB and nonclassical processes). We used isotropic-diffusion $x$-ray radiation transport and non-LTE atomic physics on the Au case.

Full-Scale Targets. Figure 2-35(a) shows a target with 1- $\mu$ g of cryogenic D-T fuel, a Au-Ni tamper, a CHOW (carbon, hydrogen, oxygen, tungsten) pusher, and a Be ablator; Fig. 2-35(b) shows a typical time-power profile assumed for the laser. As noted above, the target design is unoptimized. Each of the $\mathrm{Au}$ and $\mathrm{Ni}$ tamper shells has an aspect ratio (shell radius/shell thickness) of about 20. Approximately $20 \%$ of the Be ablator (by mass) remains unablated when the CHOW pusher attains its peak implosion velocity. We can replace the Au-Ni tamper with a single lower-density, high- $Z$ shell (such as $\mathrm{Ag}$ or $\mathrm{Pb}$ ) and convert some of the excess Be ablator into $\mathrm{CHOW}$ pusher; these modifications would reduce the aspect ratios of the tamper and pusher shells to less than 10.

Table 2-8 shows the simulation results of this target for varying input laser conditions. The values for $\mathrm{F}(\mathrm{HOT})$ and $\mathrm{T}(\mathrm{HOT})$ were determined by the normal LASNEX parameters. In each calculation, the D-T thermonuclear burn was turned off to determine the no-burn fuel conditions and turned on to obtain the target yield. The reference calculation $A$ shows that a thermonuclear yield of $160 \mathrm{~kJ}$ can be obtained; however, very good implosion symmetry is required, as indicated by the high $C R$ at mix time. A slight reduction in the input laser energy in calculation B results in an increase in the fuel $\rho \mathrm{R}$ at mix time, to about

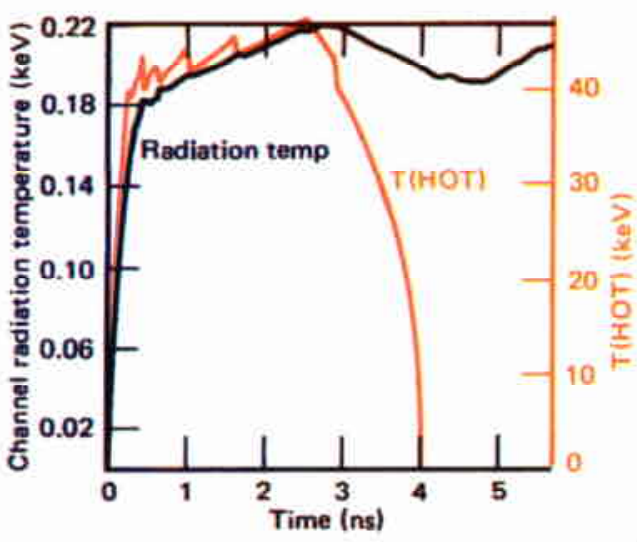

Fig. 2-36. Temporal profiles of the channel radiation temperature and T(HOT) for calculation D (see Table 2-9).

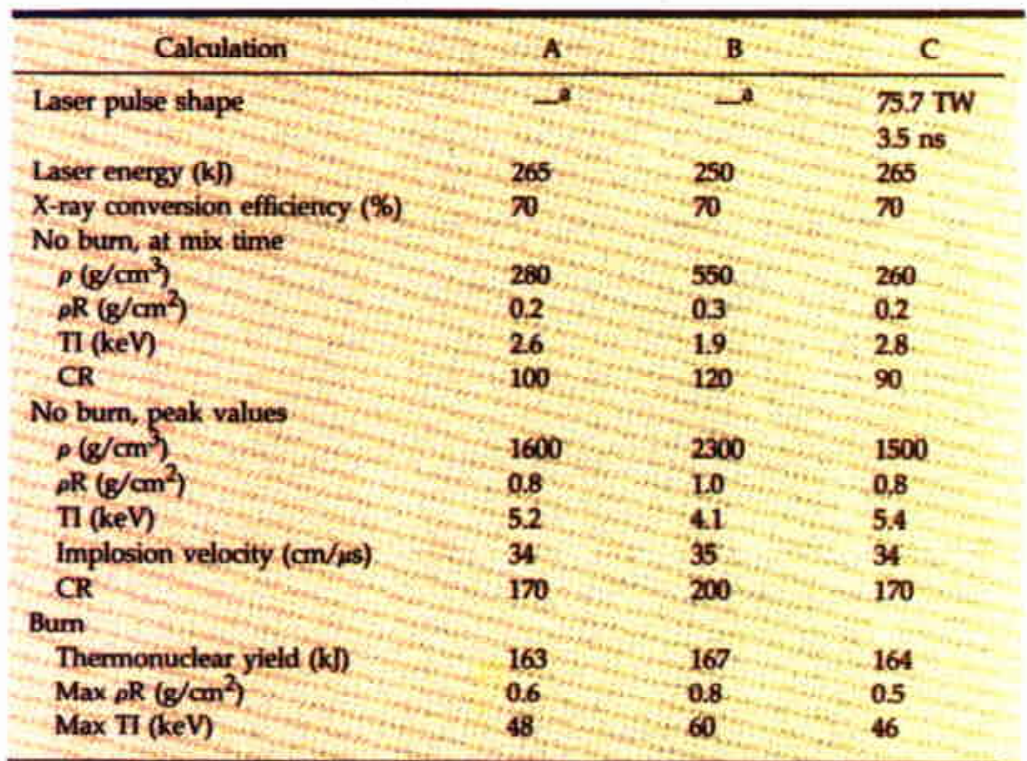

asee Fig. 2-35(b).

$0.3 \mathrm{~g} / \mathrm{cm}^{2}$. This improvement in $\rho \mathrm{R}$ is compensated by a lowering of the fuel temperature, TI. In calculation C, a 3.5-ns-duration, constant-power (75.7 TW) laser profile was used. A comparison of the results of calculations $A$ and $C$ shows that the target performance is insensitive to this change in the incident laser pulse shape.

We subjected the reference target (calculation A) to various levels of suprathermal electron preheat. A peak value of $50 \mathrm{keV}$ for T(HOT) and values of 15 and $30 \%$ for F(HOT) were imposed, respectively, in calculations D and E (Table 2-9). The effect of the suprathermal electron preheat on the target performance is clearly indicated in calculation $\mathrm{E}$ by the lower fuel density and $\rho \mathrm{R}$. We note that the 56 to $57 \%$ $x$-ray conversion efficiencies obtained in these simulations are in excellent agreement with recent $0.5-\mu \mathrm{m}$-wavelength Au-disk experimental results at comparable laser intensities. ${ }^{8}$ Figure 2-36 shows the temporal
Table 2-8. Imploded fuel parameters of a cryogenic D-T target for three input laser conditions. 
profiles of the channel radiation temperature and $T(H O T)$ in calculation D. The spikes on the $\mathrm{T}(\mathrm{HOT})$ profile arise as follows. When one of the discrete zones used to simulate the Au case expanded into the channel, its density dropped below the critical density for the laser radiation, and the laser light was allowed to penetrate into the next zone. The resulting small increase in radius of the critical-density surface (lower laser intensity) caused a decrease in the $\mathrm{T}(\mathrm{HOT})$ value and created the observed oscillations. The $x$-ray time-temperature profile, reaching a peak value of about $220 \mathrm{eV}$, is representative of the distributions obtained in these simulations. The slow rise in the radiation temperature after about $4.7 \mathrm{~ns}$ was caused by the stagnation of the $\mathrm{Au}$ and suprathermal-electron preheat on fuel compression.

\begin{tabular}{|c|c|c|c|}
\hline Calculation & A & D & E \\
\hline F(HOT) (\%) & - & 15 & 30 \\
\hline Peak T(HOT) (keV) & - & 50 & 50 \\
\hline$X$-ray conversion efficiency $(\%)$ & 70 & 57 & 56 \\
\hline \multicolumn{4}{|l|}{ No burn, at mix time } \\
\hline$\rho\left(\mathrm{g} / \mathrm{cm}^{3}\right)$ & 280 & 400 & 140 \\
\hline$\rho R\left(\mathrm{~g} / \mathrm{cm}^{2}\right)$ & 0.2 & 0.2 & 0.1 \\
\hline $\mathrm{TI}(\mathrm{keV})$ & 26 & 22 & 25 \\
\hline CR & 100 & 100 & 80 \\
\hline & \multicolumn{3}{|c|}{ No bum, peak values } \\
\hline$p R\left(g / \mathrm{cm}^{2}\right)$ & 0.8 & 0.8 & 0.6 \\
\hline $\mathrm{TI}(\mathrm{keV})$ & 5.2 & 5.1 & 5.0 \\
\hline Implosion velocity $(\mathrm{cm} / \mathrm{\mu s})$ & 34 & 34 & 32 \\
\hline CR & 170 & 170 & 150 \\
\hline \multicolumn{4}{|l|}{ Bum } \\
\hline Thermonuclear yield (k) & 163 & 166 & 152 \\
\hline $\operatorname{Max} \rho R\left(g / \mathrm{cm}^{2}\right)$ & 0.6 & 0.7 & 0.4 \\
\hline $\operatorname{Max} \mathrm{TI}(\mathrm{keV})$ & 48 & 57 & 34 \\
\hline Calculation & A & $\mathbf{F}$ & G \\
\hline F(HOT $(\%)$ & -103 & - & 10 \\
\hline Peak T(HOT) (keV) & $-i \quad$ & - & 50 \\
\hline D-T fuel & Solid & Gas & Gas \\
\hline \multicolumn{4}{|l|}{ No burn, at mix time } \\
\hline$p\left(\mathrm{~g} / \mathrm{cm}^{3}\right)$ & 280 & 120 & 160 \\
\hline$\rho R\left(g / \mathrm{cm}^{2}\right)$ & 0.2 & 0.2 & 0.2 \\
\hline II (keV) & 26 & 5.0 & $4.8 \times 6$ \\
\hline $\mathrm{CR}$ & 100 & 80 & 80 \\
\hline \multicolumn{4}{|l|}{ No burn, peak yalues } \\
\hline$\rho\left(g / \mathrm{cm}^{3}\right)$ & 1600 & 740 & 700 \\
\hline$\rho R\left(g / \mathrm{cm}^{2}\right)$ & 0.8 & 0.5 & 0.5 \\
\hline$\pi(\mathrm{keV})$ & 5.2 & 5.6 & 5.5 \\
\hline Implosion velocity (cm/ $\mathrm{ms}$ ) & 34 & 32 & $31-6$ \\
\hline CR & 170 & 130 & 130 \\
\hline \multicolumn{4}{|l|}{ Bum } \\
\hline Thermonuclear yield $(\mathrm{k})$ & 163 & 114 & 108 \\
\hline $\operatorname{Max} p R\left(\mathrm{~g} / \mathrm{cm}^{2}\right)$ & 0.6 & 03 & 035 \\
\hline Max II (keV) & 48 & 19. & 18 \\
\hline
\end{tabular}

Table 2-10. Imploded-fuel parameters

when cryogenic D-T is replaced by gaseous D-T of equal mass.
Be in the channel, but this increase in the channel temperature occurred after the pusher had reached its peak implosion velocity at $4.4 \mathrm{~ns}$. Consequently, the target performance was not improved by this additional drive.

A gaseous D-T target is less troublesome to fabricate than a cryogenic tanget. Consequently, in calculations F and G (Table 2-10), we replaced the cryogenic D-T fuel used in calculation $A$ with an equal mass of D-T gas (initial density $=26.8 \mathrm{mg} / \mathrm{cm}^{3}$ ) to examine this design option. The higher ion temperatures at mix time are obtained at the expense of lower fuel densities, and there are moderate reductions in the convengence ratios and thermonuclear yields. The gaseous D-T targets can be used as test beds for the more advanced cryogenic Apollo targets.

To date, only $\mathrm{CH}$ or $\mathrm{CF}_{2}$ have been fabricated into ablator materials and used in ICF target experiments; therefore, for calculations $\mathrm{H}$ and I (Table 2-11), we substituted $\mathrm{CH}$ ablator for the Be ablator used in calculation $\mathrm{A}$. Since $\mathrm{CH}$ has a higher $\mathrm{Z}$ than $\mathrm{Be}$, it is a less efficient ablator; consequently, the $\mathrm{CH}$ ablator masses in calculations $\mathrm{H}$ and $\mathrm{I}$ are only one-half the Be ablator mass in calculation A. Degradations in the target performance resulting from the substitution of $\mathrm{CH}$ for Be are not very severe. We now have more flexibility in the choice of the ablator material.

0.55-Scale Targets. The full-scale targets described above require much more laser energy than will be available in the near future, so we have done a preliminary study of a 0.55 -scale, 50-kJ target design. The energy requirement of $50 \mathrm{~kJ}$ is well below the possible output of the Nova Phase I frequency-converted Nd:glass laser. For the 0.55-scale target design, all of the dimensions of the target shown in Fig. 2-34 were reduced by a factor of 0.55 , and some of the excess Be ablator was converted into CHOW pusher. This increased pusher thickness reduces the shell aspect ratio to a more conservative value of 7.8 , as compared with a value of about 10.9 for the full-scale target. The temporal pulse shape of the incident laser radiation is shown in Fig. 2-37(b). At peak laser power (32 TW), the laser intensity on the Au case is about $9 \times$ $10^{13} \mathrm{~W} / \mathrm{cm}^{2}$. Table 2-12 shows the simulation results for both cryogenic and gaseous 
D- $\mathrm{T}$ target designs (calculations $\mathrm{J}$ and $\mathrm{K}$, respectively) that use a Au-Ni tamper. Peak fuel densities in excess of $600 \mathrm{~g} / \mathrm{cm}^{3}$ and thermonuclear energy yields of more than $1 \mathrm{~kJ}$ are obtained.

As noted above, the aspect ratios of the $\mathrm{Au}$ and $\mathrm{Ni}$ tamper shells are about 20.

When the layered Au-Ni tamper is replaced with a single $\mathrm{Pb}$ shell, this ratio is reduced to 8.4. Thus, the aspect ratios of the pusher and tamper in the target design shown in Fig. 2-37(a) are both less than 10. Table 2-13 shows the one-dimensional simulation results of this target filled with gaseous D-T for two suprathermal-electron flux models; there is no significant difference between the results of these two calculations. The large thermonuclear energy yields shown in Table 2-13 should allow the implosion characteristics to be diagnosed by neutron imaging techniques.

We recognize that some of the components in these initial target designs may create near-term target fabrication problems, and more design effort will certainly be required to eliminate these difficulties. Nevertheless, the results of these preliminary one-dimensional LASNEX simulations suggest that an Apollo target can produce a very high compressed-fuel density and a significant thermonuclear energy yield when exposed to the frequency-converted $(2 \omega$ to $3 \omega$ ) radiation output of the Nova Phase I Nd:glass laser system.

\section{Author: Y. Pan}

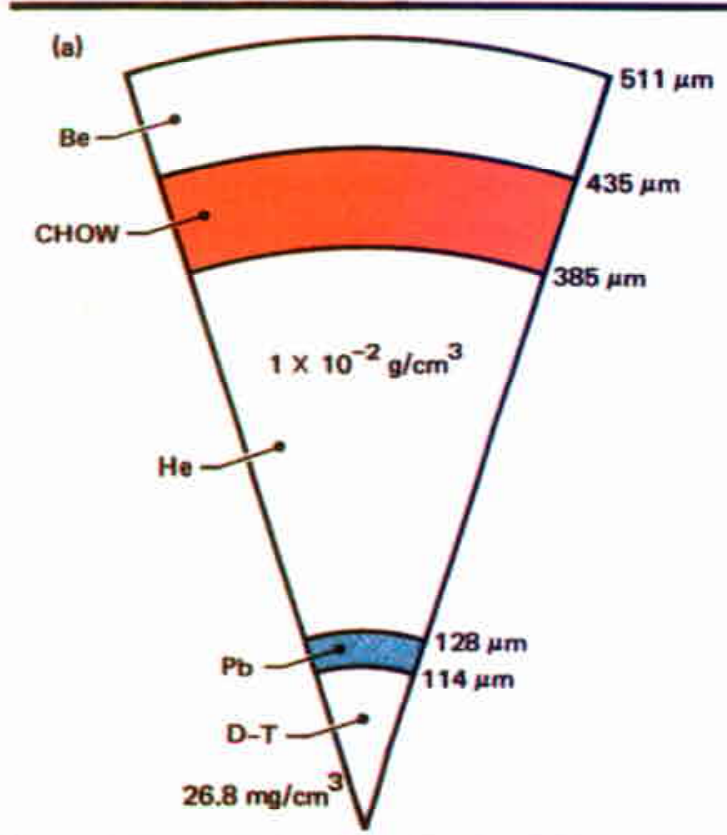

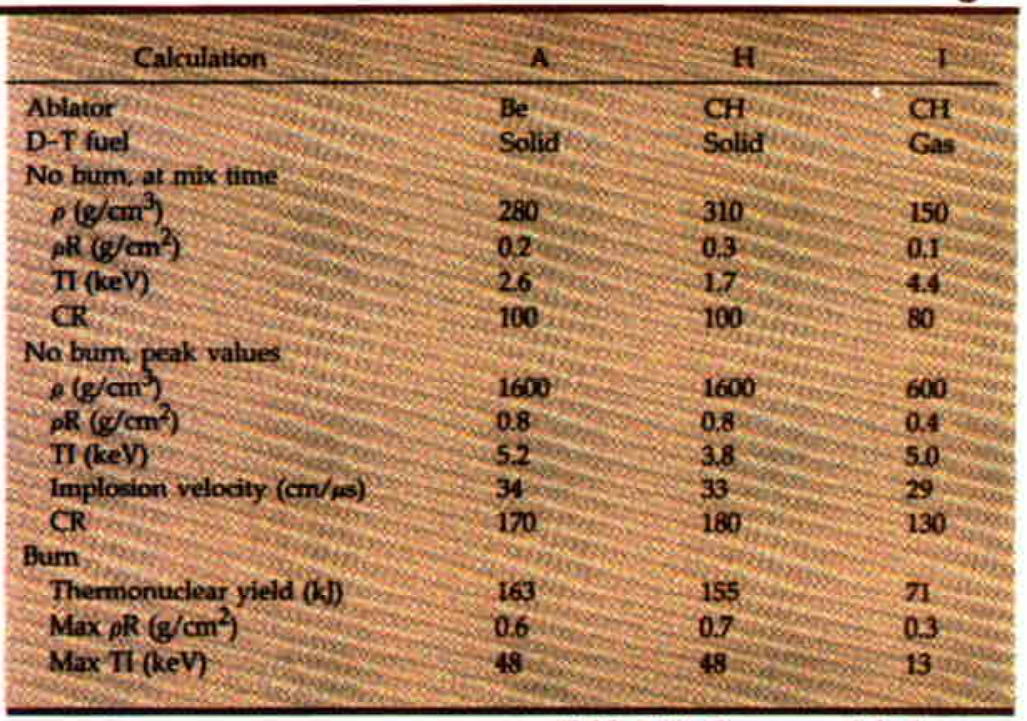

Table 2-11. Compressed-fuel con ditions when Be ablator is replaced by $\mathrm{CH}$ having one-half the Be mass.

\begin{tabular}{|c|c|c|}
\hline$D-T$ fuel & Solid & Gas \\
\hline \multicolumn{3}{|l|}{ No burn, at mix time } \\
\hline $\begin{array}{l}p\left(\mathrm{~g} / \mathrm{cm}^{3}\right) \\
\rho R\left(\mathrm{~g} / \mathrm{cm}^{2}\right)\end{array}$ & $\begin{array}{l}160 \\
0.08\end{array}$ & \\
\hline$\pi(\mathrm{keV})$ & 24 & 3.9 \\
\hline CR & 80 & 80 \\
\hline \multicolumn{3}{|l|}{ No bum, peak values } \\
\hline$p\left(\mathrm{~g} / \mathrm{cm}^{3}\right)$ & 1050 & 640 \\
\hline$\rho R\left(g / \mathrm{cm}^{2}\right)$ & 0.3 & 02 \\
\hline $\mathrm{Tl}(\mathrm{keV})$ & 4.5 & 4.5 \\
\hline Implosion velocity $(\mathrm{cm} / \mu \mathrm{s})$ & 30 & 30 \\
\hline $\mathbf{C R}$ & 150 & 130 \\
\hline \multicolumn{3}{|l|}{ Bum } \\
\hline Thermonuclear yield (k) & 4.5 & 1.4 \\
\hline $\operatorname{Max} p R\left(\mathrm{~g} / \mathrm{cm}^{2}\right)$ & 0.3 & 0.2 \\
\hline $\operatorname{Max} \pi(\mathrm{keV})$ & 8.4 & 5.5. \\
\hline
\end{tabular}

Table 2-12. Implodedfuel parameters for Nova Phase I targets filled with solid and gaseous D-T fuel. (b)

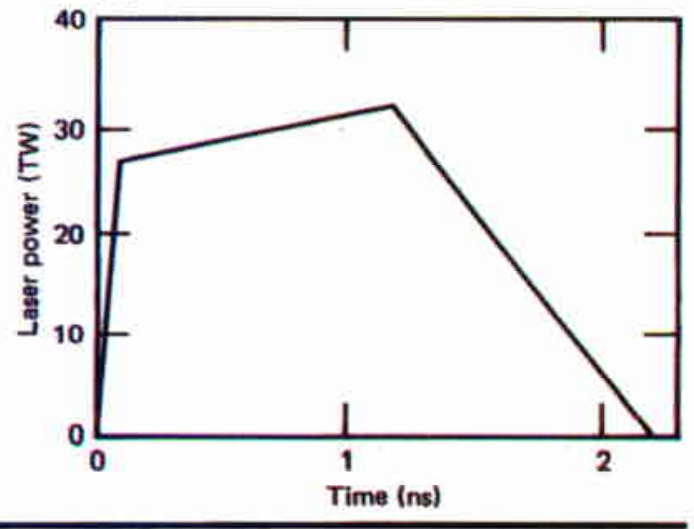

Fig. 2-37. (a) Onedimensional diagram of a 0.25 - to $0.50-\mu \mathrm{m}$ wavelength Apollo target scaled for Nova Phase L. (b) Pulse shape of the incident laser beam. 


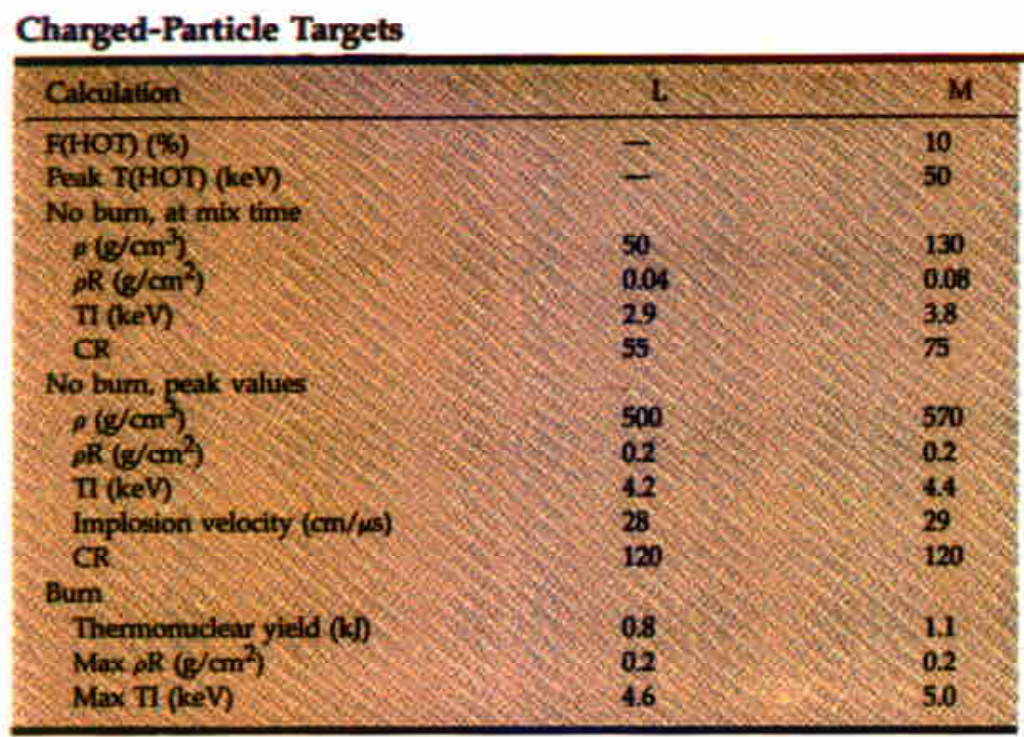

Table 2-13. Compressed-fuel parameters of a Nova Phase I target (Fig. 2-37)

Fig. 2-38. Target gain for two suprathermal-electron preheat vs driving energy for models (gaseous D-T). double-shell targets.

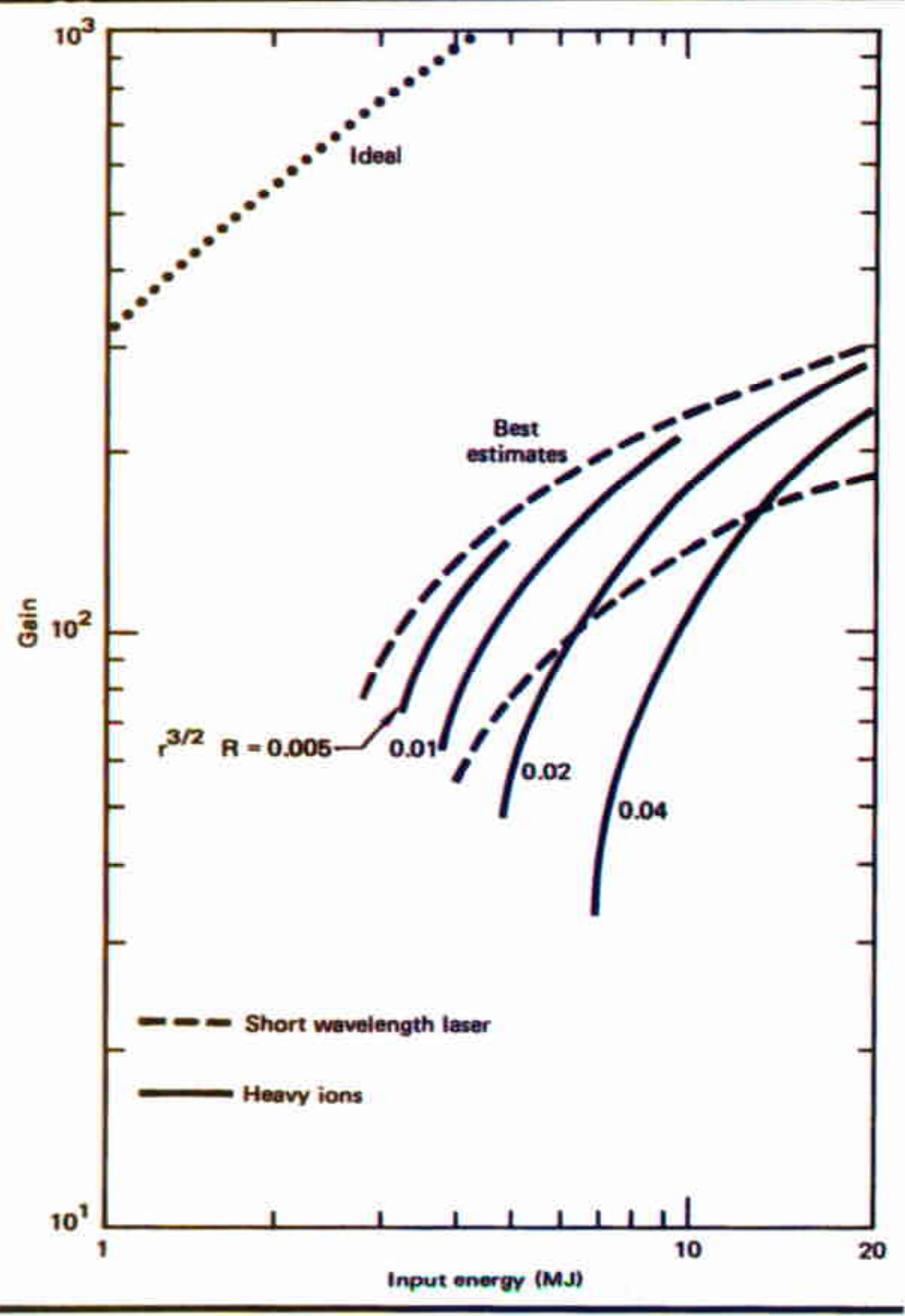




\section{Laser-Plasma \\ Simulation}

Overview of Laser-Plasma

Coupling in Radiation-Driven

\section{Targets}

Laser-plasma coupling in hohlraums has a number of special features that are important to consider. First, the absorption of laser light is much improved, as compared with analogous open targets. Because light is focused into a can through several holes, the light can undergo multiple interactions with the high- $Z$ plasma within the can. Not surprisingly, the absorption of $1.06-\mu \mathrm{m}$ light is quite high: a measured fractional absorption of 70 to $80 \%$ is typical (see "Cairn Scaling" in Section 4).

Second, compared to open targets, Brillouin scatter is much less of an absorp- 
tion issue in hohlraums. ${ }^{12}$ Our calculations have indicated that the Brillouin instability scatters light over a broad range of angles and, hence, is dangerous as a loss mechanism mainly near the entrance holes where the plasma density is rather low. If we use LASNEX-calculated plasma conditions for a typical experiment with a Caim hohlraum, and consider the plasma within one diameter of the entrance hole, our nonlinear theory estimates a Brillouin reflectivity of $\approx 15 \%$. This result is consistent with observed data, which do indicate that the nonabsorbed light is strongly red-shifted (see "Cairn Scaling" in Section 4). Similar estimates for the nominal hohlraum to be irradiated with Nova predict a reflectivity of $\simeq 30 \%$ when $1.06-\mu \mathrm{m}$ light is used. An improved understanding of the plasma conditions, the detailed angular distribution of the scatter, and the competition of the scatter with other coupling processes is needed to quantitatively improve this prediction. A change in the distribution of light within the hohlraum is expected to be an important consequence of Brillouin scatter.

A third, and key, feature of laserirradiated hohlraums is the development of a large volume of underdense plasma as plasma blows off the walls and accumulates within the can. Large regions of underdense plasma can alter the relative importance of absorption processes, since a number of instabilities take place in this plasma. Of particular importance to target design is the generation of very energetic electrons, either directly via the Raman and two-plasmon decay instabilities or indirectly by the filamentation instability, which strongly increases the intensity of the light. The generation of copious amounts of hot electrons, as well as the predicted sizable levels of Raman-scattered light, have, in fact, been observed in many hohlraum experiments. As discussed in "Hot-Electron Generation in Hohlraums," below, our calculations of hot-electron generation via instabilities in the underdense plasma within the hohlraum compare favorably with observations. These calculations have also led to the formulation of a simple model for scaling of both the number and the temperature of hot electrons with the hohlraum size and with the energy, pulse length, and wavelength of the irradiation (see "HotElectron Scaling in Hohlraums and Its Im- pact on Nova Ignition-Capsule Design," earlier in this section). A simple model of the instability-induced heating has been incorporated into LASNEX, our target design code.

Our calculations continue to emphasize that the laser-plasma coupling is significantly improved when shorter-wavelength light is used to irradiate hohlraums. ${ }^{12}$ Collisional absorption is enhanced, and the various instability thresholds increase as the wavelength of the light decreases. More importantly, a hohlraum of a given size fills with plasma whose density is a smaller fraction of the critical density of shorter wavelength light, thus reducing the efficiency of the instabilities in that plasma. Preliminary experiments in which small hohlraums are irradiated with 0.53 - and $0.35-\mu \mathrm{m}$ light are consistent with these predictions. More experiments with larger hohlraums irradiated with more energy in a Ionger pulse (i.e., longer than $600 \mathrm{ps}$ ) are clearly needed to check and extend these promising results on wavelength scaling of laser-plasma coupling. It is also very important to measure the plasma conditions within the hohlraums to test the critical assumptions underlying our coupling calculations.

Author. W. L. Kruer

\section{Hot-Electron Generation in Hohlraums}

Introduction. In radiation-driven implosions, ${ }^{13}$ a pulse of laser light is directed into a cylindrical gold can (hohlraum), where the laser energy is absorbed and a portion of the absorbed energy is converted into $x$ rays. The mechanisms by which the laser light is absorbed within the hohlraum affect both the efficiency with which the laser light is converted into $x$ rays and, most importantly, the magnitude of the undesired preheat of the capsule by high-enengy (hot) electrons. From a coupling viewpoint, a key feature of laser-irradiated hohlraums is that they fill with large regions of underdense plasma. As the can is heated and $\mathbf{x}$ rays are generated, plasma blows off from the walls, even from those parts of the walls not directly illuminated by the laser light, and this plasma accumulates within the enclosed can. The presence of a large volume 
of underdense plasma can lead to absorption of laser energy by electrons with a very high temperature, since coupling processes in the lower-density plasma are then enhanced. The most important mechanisms for generating hot electrons in the underdense plasma are the Raman, twoplasmon-decay, and filamentation instabilities. We will first give a theoretical discussion of these mechanisms, and we will then consider a specific application of theory to recent experiments made with laserirradiated hohlraums.

Mechanisms That Produce Hot Electrons. The generation of hot electrons (sometimes called suprathermal electrons) is a common feature of laser-plasma coupling and is a signature of absorption via collective processes. In this coupling, laser light excites plasma waves that, in turn, transfer their energies to electrons by accelerating them. Since plasma waves preferentially accelerate the faster (resonant) electrons, a high-energy tail of electrons is generated.

In past experiments, with only small regions of underdense plasmas, ${ }^{14}$ the generation of hot electrons was principally attributed to resonance absorption, ${ }^{15}$ a collective-absorption process that takes place at the critical density $\left(n_{c}\right)$. Simulations ${ }^{16}$ of resonance absorption typically showed about $30 \%$ absorption into a tail of hot electrons with a temperature $\theta_{\mathrm{h}} \simeq 10^{-5}$ $T_{e}^{0.25}\left(D_{\mu}^{2}\right)^{0.39}$, where $T_{e}$ is the background electron temperature in $\mathrm{keV}, \mathrm{I}$ is the intensity in $W / \mathrm{cm}^{2}$, and $\lambda_{\mu}$ is the wavelength in $\mu \mathrm{m}$.

The presence of large regions of plasma with densities less than critical can introduce new avenues for hot-electron generation by the

- Raman instability.

- Two-plasmon-decay instability.

- Filamentation instability.

In the lower-density plasma, the Raman ${ }^{17}$ and two-plasmon-decay ${ }^{18}$ instabilities generate electron plasma waves, while filamentation of the laser light ${ }^{19}$ as it propagates through the underdense plasma can greatly increase the intensity of the light. The latter effect both reduces inverse bremsstrahlung and makes resonance $a b$ sorption and other collective-absorption mechanisms more efficient.

Other possible mechanisms for hotelectron generation are parametric instabil- ities near the critical density, absorption on ion turbulence driven by heat-flow instabilities, and continued reheating of electrons whose flow is inhibited by large selfgenerated magnetic fields. These processes have not recently been given much attention, but are difficult to completely rule out in view of our meager knowledge of plasma conditions and electron transport in hohlraums.

Two-Plasmon-Decay and Raman Instabilities. The two-plasmon-decay ( $\left.2 \omega_{\text {pe }}\right)$ and Raman instabilities can most simply be described as the resonant decay of a light wave either into two electron plasma waves or into an electron plasma wave and a scattered light wave. From the frequencymatching conditions, it is apparent that the $2 \omega_{\mathrm{pe}}$ instability occurs near $0.25 \mathrm{n}_{\mathrm{cr}}$ and the Raman instability takes place at a density $\$ 0.25 n_{\mathrm{cr}}$ For either of these instabilities, absorption is most efficient near $0.25 \mathrm{n}_{\mathrm{cr}}$ where the instability is absolute. The threshold intensity $\left(\mathrm{I}_{\mathrm{T}}\right)$ of these instabilities is usually determined by density gradients, which interfere with the resonant coupling. These threshold intensities are

$\mathrm{I}_{\mathrm{T}} \approx 5 \times 10^{15} \frac{\theta_{\mathrm{kev}}}{\mathrm{L}_{\mu} \lambda_{\mu}}$

for $2 \omega_{p e}$ instability, and

$\mathrm{I}_{\mathrm{T}} \approx \frac{5 \times 10^{17}}{\lambda_{\mu}^{2 / 3} \mathrm{~L}_{\mu}^{4 / 3}}$

for Raman instability near $0.25 n_{c r}$ In Eqs. (19) and (20), $I_{T}$ is the intensity in $W / \mathrm{cm}^{2}, L_{\nu}\left(\lambda_{\nu}\right)$ is the local density-gradient length (wavelength) in $\mu \mathrm{m}$, and $\theta_{\mathrm{keV}}$ is the background electron temperature in keV. Collisional absorption gives a lower limit on the threshold intensity, which can be estimated by replacing $L_{\mu}$ with the absorption length ( $\mathrm{L}_{\mathrm{ab}}$ ) for light propagating up to $0.25 \mathbf{n}_{\mathrm{cr}}$ For a gold plasma, this absorption length, in $\mu \mathrm{m}$, is $L_{\text {abs }} \simeq 500 \theta_{\mathrm{kev}} \lambda_{\mu}^{2}$.

To date, our two-dimensional particle simulation $s^{20}$ indicate that, above the instability threshold, the instabilities near $0.25 n_{c}$ can be characterized by an absorption length $\left(\ell_{\mathrm{a}}\right)$ of $\ell_{\mathrm{a}} \sim 2 \mathrm{~d} / \gamma_{\sigma}$, where $\mathrm{c}$ is the velocity of light and $\gamma_{\circ}$ is the maximum linear growth rate. Hence $\ell_{\mathrm{a}} \sim 2 \times 10^{9} / \mathrm{I}^{1 / 2}$, 
where $\ell_{\mathrm{a}}$ is in $\mu \mathrm{m}$ and $\mathrm{I}$ is in $W / \mathrm{cm}^{2}$. As an example, for light with an intensity of $10^{15}$ $\mathrm{W} / \mathrm{cm}^{2}, \ell_{a}-60 \mu \mathrm{m}$. These simulations ${ }^{20}$ also show the generation of hot electrons with an effective temperature of 75 to 150 $\mathrm{keV}$, weakly dependent on intensity.

At densities below $\sim 0.2 \mathrm{n}_{\mathrm{cr}}$ the twoplasmon-decay instability is no longer operative, but the Raman instability (and its kinetic analog) persists. Simulations of Raman backscatter in this regime ${ }^{21}$ show that the absorption length becomes longer and the hot electrons produced become cooler as the density decreases. For example, in one-dimensional simulations in which $1.06-\mu \mathrm{m}$ light with an intensity of $2.5 \times$ $10^{16} \mathrm{~W} / \mathrm{cm}^{2}$ is propagated through a slab of plasma having an electron density varying between 0.075 and $0.125 \mathrm{n}_{\mathrm{cr}}$ in $127 \mu \mathrm{m}$ and an electron temperature of $10 \mathrm{keV}$, about $11 \%$ of the light is absorbed into hot electrons having a temperature of about 32 $\mathrm{keV}$. Our results show that the heatedelectron temperature can be estimated as $m v_{p}^{2} / 2$, where $v_{p}$ is the phase velocity of the plasma wave associated with the Raman scatter. Note that Raman sidescatter is then expected to produce a heated-electron temperature about twice as high as that produced by backscatter, since the wavelength of the plasma wave generated by sidescatter is about $\sqrt{2}$ longer than that due to backscatter. Sidescatter occurs preferentially out of the plane of polarization and has approximately the same density gradient threshold as does backscatter near $0.25 n_{\text {rr }}$

It is actually Raman forward scatter ${ }^{21,22}$ that produces the most energetic electrons, since the plasma wave associated with forward scatter has a very long wavelength and, hence, a high phase velocity. Simulations with large slabs of plasma having a uniform density of $0.1 n_{c}$ have shown heated electrons with a very hard tail extending out to energies of several MeV due to forward scatter. Fortunately, forward scatter has a weaker growth rate than does either backscatter or sidescatter (in the absence of Landau damping), and forward scatter also has a much higher intensity threshold due to density gradients. In particular, $I_{T} \sim 5 \times 10^{17} n_{c c} / n_{p} / L_{\mu} \lambda_{\mu}$ for $\mathrm{n} \leqslant$ $0.1 n_{c r}$. Here, $I_{T}$ is in $W / \mathrm{cm}^{2}, n_{c r}\left(n_{p}\right)$ is the critical (plasma) density, and $L_{\mu}\left(\lambda_{\mu}\right)$ is the local density-gradient scale length (wavelength of the light) in $\mu \mathrm{m}$. Hence, efficient forward scatter requires high-intensity light, quite large regions of rather uniform plasma, or both. Our theory indicates that Raman forward scatter is below threshold for the LASNEX-computed plasma conditions in the nominal Nova hohlraum.

The use of laser light with wavelengths shorter than $1.06 \mu \mathrm{m}$ is predicted to decrease the generation of hot electrons in hohlraums due to the $2 \omega_{\text {pe }}$ and Raman instabilities. As the wavelength decreases, - The intensity thresholds due either to collisions or to density gradients are higher.

- The hohlraum fills to a smaller fraction of the critical density if the irradiation conditions and the size of the hohlraum are held constant; hence, there is simply much less plasma with densities of $\sim 0.1$ to $0.25 \mathrm{n}_{\mathrm{c}}$ available for the instabilities that generate hot electrons.

Filamentation. Filamentation of the incident laser light is a volumetric process that can indirectly lead to the generation of very-high-energy electrons via resonance absorption or other collective-absorption mechanisms. The physics of filamentation is straightforward. A local increase in the laser-light intensity pushes plasma aside via the ponderomotive force, creating a density depression that refracts the light inward, further increasing its intensity. The net result is the breakup of the incident light beam into intense filaments. The filamentation instability can be conveniently characterized by a spatial gain coefficient, $K_{\text {, }}$ which, for the most unstable filament, is

$\mathrm{K}_{\mathrm{f}} \simeq \frac{\pi}{2} \frac{1}{\lambda_{0}} \frac{\mathrm{n}_{\mathrm{p}}}{\mathrm{n}_{\mathrm{cr}}}\left(\frac{\mathrm{v}_{\mathrm{os}}}{\mathrm{v}_{\mathrm{e}}}\right)^{2} /\left(1+\frac{\mathrm{T}_{\mathrm{i}}}{\mathrm{ZT}_{\mathrm{e}}}\right)$,

where $v_{\mathrm{os}}$ is the oscillation velocity of an electron in the electric field of the light wave, $v_{e}$ is the electron thermal velocity, $n_{p}$ is the plasma density, and $T_{i}\left(T_{e}\right)$ is the ion (electron) temperature. Note that, for a hohIraum filled with plasma with a given mean density of $n_{p}$ and a temperature of $T_{e}$ $K_{f}$ scales as $\lambda_{\alpha}^{3}$ which indicates that shorter-wavelength light is less sensitive to filamentation.

One possible effect of filamentation would be to decrease the inverse bremsstrahlung and enhance both the amount of resonance absorption and the temperature of the resonantly heated electrons. A crude 
estimate is that the light can filament to an intensity sufficient to expel plasma from the filament: $\mathrm{D}_{\mu}^{2} \sim 10^{16} \theta_{c}$ where $\mathrm{I}$ is the intensity in $W / \mathrm{cm}^{2}, \lambda_{\mu}$ is the wavelength in $\mu \mathrm{m}$, and $\theta_{\mathrm{c}}$ is the background plasma temperature in keV. According to LASNEX calculations of typical hohlraums irradiated with 1.06- $\mu \mathrm{m}$ light, ${ }^{23} \theta_{\mathrm{c}}$ is set by inverse bremsstrahlung and is typically $\sim 5$ to $10 \mathrm{keV}$ in the underdense plasma. Hence, the filamented intensity can be $\sim 5 \times 10^{16}$ $\mathrm{W} / \mathrm{cm}^{2}$. Resonance absorption of a filament with this intensity would produce hot electrons with a temperature of $\sim 100 \mathrm{keV}$. Since generation of filaments is a volumetric process, this mechanism for the generation of hot electrons also depends on the filling of the hohlraum.

\section{Application of Theory to Recent} Hohlraum Experiments. One major difficulty in calculating the coupling of laser light within hohlraums is the significant uncertainty in the plasma conditions within the hohiraum. Recognizing this uncertainty, we proceed by briefly considering the plasma conditions calculated with LASNEX for a $2.0 \mathrm{H}$ Caim hohlraum ${ }^{24}$ irradiated with a 600 -ps, 6-kJ pulse of $1.06-\mu \mathrm{m}$ light. In this two-dimensional LASNEX calculation, the standard model developed from the high-Z disc experiments ${ }^{25}$ was used.

Figure 2-62 shows the contours of plasma density at the peak of the laser pulse. Note that the critical-density contour is still close to the wall, but that a lange fraction of the volume of the can is filled with plasma to a density of 0.1 to $0.4 \mathrm{n}_{\mathrm{cr}}$. The corresponding temperature contours show that the

Fig. 2-62. Contours of plasma density $\left(\mathbf{n} / \mathbf{n}_{\mathrm{c}}\right)$ at the peak of the laser pulse for $2.0 \mathrm{H}$ Cairn.

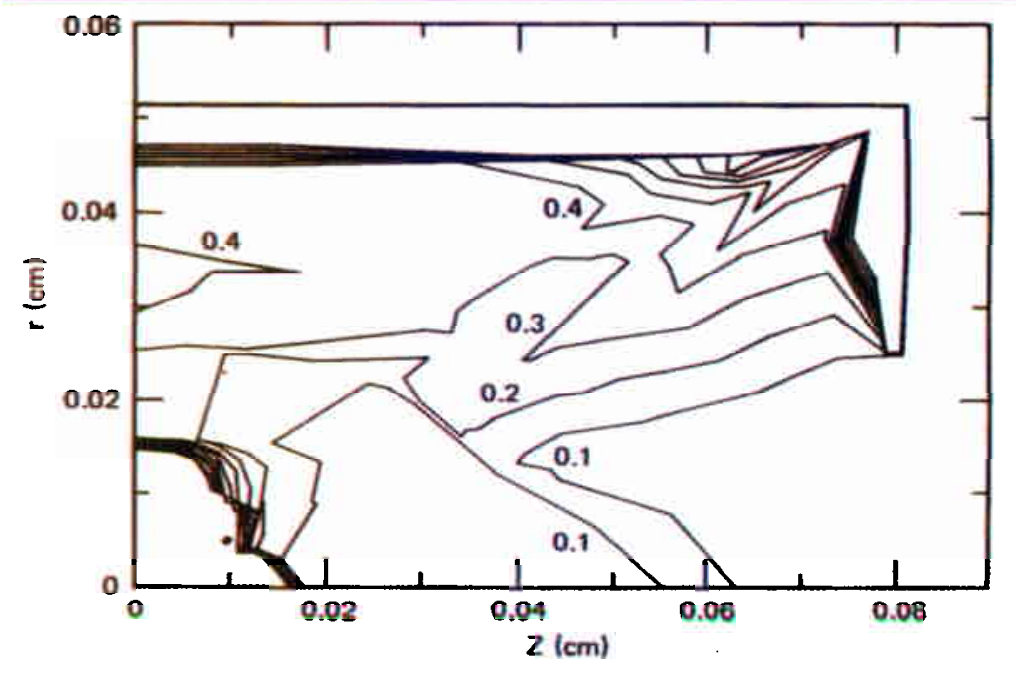

background electron temperature is about 6 $\mathrm{keV}$ in this density region. The incident laser-light intensity, determined by ray tracing, ranges from $\sim 5 \times 10^{16} \mathrm{~W} / \mathrm{cm}^{2}$ at the entrance hole to $\sim 3 \times 10^{16} \mathrm{~W} / \mathrm{cm}^{2}$ in the region of $0.25 n_{\mathrm{cr}}$ to $\sim 3 \times 10^{15} \mathrm{~W} / \mathrm{cm}^{2}$ near the critical density.

For the plasma and irradiation conditions near $0.25 n_{c r}$, both the $2 \omega_{p e}$ and Raman instabilities are far above their intensity thresholds, which, in this example, are all about $3 \times 10^{14} \mathrm{~W} / \mathrm{cm}^{2}$. In addition, we estimate that several absorption lengths near $0.25 n_{c r}$ are available, indicating that we can expect sizable absorption by these instabilities. To investigate the coupling, we carried out a one-dimensional particle simulation, which includes both Raman and Brillouin backscatter. In this simulation, a plasma slab with density rising from 0.1 to $0.3 n_{a r}$ in $127 \mu \mathrm{m}$ and with an electron temperature of $6 \mathrm{keV}$ was irradiated with 1.06- $\mathrm{mm}$ light having an intensity of $3 \times 10^{16} \mathrm{~W} / \mathrm{cm}^{2}$. About one-half of the light was absorbed via the Raman instability into electrons with a temperature of $\sim 100 \mathrm{keV}$; less than $10 \%$ of the light was reflected by the Brillouin instability. For these specific plasma conditions, we have not yet carried out twodimensional simulations that include the $2 \omega_{\text {pe }}$ instability. However, based on previous two-dimensional simulations, we expect that a mix of the $2 \omega_{p e}$ and Raman instabilities will result in an even larger absorption into electrons with a similarly high temperature. The two-dimensional calculations emphasize that measuremients of the Raman-scattered light provide an underestimate of the heating, since the $2 \omega_{\text {pe }}$ instability also contributes to the heating and part of the Raman-scattered light is absorbed on the way out of the plasma.

Our calculations compare favorably with the results of experiments ${ }^{13}$ in which a $2.0 \mathrm{H}$ Cairn hohlraum was irradiated with a 6-k], 600 -ps pulse of $1.06-\mu \mathrm{m}$ light. Measurements of the high-energy $x$ rays indicated that about one-half of the laser energy was absorbed in hot electrons with a temperature of about $60 \mathrm{keV}$. We note that the hot-electron temperature inferred from $x$ rays - a time and space average-is typically lower by a factor of up to 2 than the actual hot-electron temperature at the peak of the laser pulse. In addition, a sizable amount of scattered light with a frequency 
in the neighborhood of $\omega_{0} / 2$ was observed, providing direct evidence for the efficient generation of Raman instability.

Other experiments in which the can size and the input energy were varied show a strong variation in the fraction of the energy absorbed in hot electrons. LASNEX calculations of these experiments ${ }^{26}$ indicate that the fraction absorbed into hot electrons varies as the amount of laser energy remaining in the pulse after a sizable part of the can fills to densities near $0.25 n_{c r}$ Observed delays in the time of onset of the hard $x$-ray signals are consistent with this interpretation. There also appears to be the expected correlation between the level of the hard $x$-ray fluences and the level of the Raman-scattered light. Finally, our early experiments, in which small hohlraums were irradiated with $0.53-\mu \mathrm{m}$ laser light, indicate the expected reduction in hot-electron generation.

Although we have a preliminary model for hot-electron generation in hohlraums, many important issues remain to be resolved. Our ability to reliably calculate plasma conditions and the extent of hohlraum filling is open to question due to uncertainties in electron transport; detailed measurements of these plasma conditions are crucial to the testing of critical assumptions underlying our calculations. The quantitative mix of coupling processes in langescale-length plasmas is not well understood, and processes such as filamentation need further theoretical and experimental investigations. An improved understanding of the angular distribution of Brillouin scatter and its competition with other instabilities is also needed, since this process can, at the very least, change the location of the absorption in hohlraums. Experiments in which larger hohlraums are irradiated with longer-pulse-length light are needed to test hot-electron scaling in a parameter space close to that of future Nova targets. Further experimental tests of hot-electron generation as a function of laser wavelength are also important. Finally, we need more measurements of $x$ rays with energies $Z 300$

$\mathrm{keV}$, since these $x$ rays are a signature of the electrons that lead to preheat in Nova-size targets.

Authors: W. Kruer, K. G. Estabrook, B. F. Lasinski, and A. B. Langdon 


\section{Hohlraum Studies}

Introduction

The approaches to realizing deuteriumtritium (D-T) fuel-ignition conditions using laser systems separate naturally into two categories: directly illuminated "electron conduction" driven targets and soft $x$-ray driven capsules. In the latter, $x$ radiation from the conversion of focused laser energy is contained and used to compress and ignite a physically separate component that contains thermonuclear fuel.

At LLNL, this latter approach to inertial confinement fusion, which parallels the technique used in thermonuclear weapons, is being pursued vigorously. The Caim and Apollo ${ }^{1}$ target designs have resulted in implosions in which D-T fuel was compressed to $20 \mathrm{~g} / \mathrm{cm}^{3}$ (100 times liquid D-T density) and raised to a temperature of $0.4 \mathrm{keV}$ using the Shiva laser during 1979 and 1980.

Soft $x$-ray-driven ICF targets offer an important advantage: by placing the fuel capsule inside a high- $Z$ enclosure, the effects of short-wavelength perturbations in intensity within a single laser beam or between beams are efficiently smoothed. Longwavelength perturbations are effectively smoothed by choosing an enclosure design that leads to a very symmetric implosion. Because of this smoothing, the driver beams, whether photons or particles, need not be extraontinarily smooth or uniformly arranged and can be incident on the target from only two directions. This greatly facilitates the design of a fusion reactor and explains, in large part, why Shiva's beams are arranged in two opposed clusters.

An immediately apparent disadvantage of $x$-ray driven targets is that an additional (and possibly inefficient) step has been added in driving the capsule, i.e., absorbed laser light must be converted to soft $x$ rays and transported to the capsule. Experimen$\mathrm{tal}$ and theoretical scaling studies of $x$-ray drive have been an important component of our program for the past three years. Additional information was added to our data base this year, but the general picture remains unaltered; i.e., laser pulses with wavelengths of $1.06 \mu \mathrm{m}$ and less have been able to raise Cairn hohlraums to equivalent temperatures near $200 \mathrm{eV}$.

A less obvious potential liability has been our repeated observation that hohlraums often produce fluxes of hot electrons far exceeding those seen in open geometries such as disks. In many cases laser blowoff plasmas are trapped within a hohlraum and are calculated to reach electron densities near $n_{N} / 4$ before the end of the focused laser pulses. These plasmas can result in laser light self-focusing and can support high phase-velocity electron-plasma waves. Electron wave-breaking energies reach several tens of $\mathrm{keV}$ in $1.06-\mu \mathrm{m}$ irradiated hohlraums and the flux of suprathermal electrons so produced can be a serious source of capsule preheat. This year, as shown in the following section, we significantly advanced our ability to predict and control suprathermal preheat.

Another article relates our early work with HEET (high-energy electron thermometer), a new $x$-ray fluorescence technique designed to show where suprathermal electrons are deposited in hohlraum targets. Clearly, the target designer is interested in absolute spatial and temporal preheat measurements at the capsule. The HEET technique offers an avenue for making such determinations.

This year the Shiva laser was used in experiments simulating the electron-plasma densities expected at the laser entrance aperture during Nova target irradiations. The Nova cone-angle experiments sought to test the concept of placing Nova's laser beams in an open-cone configuration. Increasing the angle of the cone improves the calculated drive uniformity in a Nova-irradiated. hohlraum, but it also increases the risk that refraction will deflect laser light away from the hohlraum entrance aperture. We used LASNEX to design a series of targets that could be irradiated by Shiva and achieve entrance-aperture plasma densities equal to and substantially higher than the highest values expected in Nova designs. The first phase of these studies, reported below, revealed no flaw in the Nova planning.

Finally, we summarized our planning and design of a $4 \omega$ UV probe beam for Novette to be used in interferometry applications to help us study plasma conditions in enclosed laser-irradiated geometries.

Authors: K. R. Manes and J. D. Lindl 


\section{Cairn Scaling}

Over the last year and a half we accumulated information on the scaling of $x$-ray drive and suprathermal preheat in laserdriven hohlraum targets. Early in 1981 we established a data base of our experimental results to facilitate the testing of hypotheses about hohlraum modeling. We chose a relational data-base system and compiled an expandable list of about 60 parameters that describes the target's behavior. To date, partial data for over $\mathbf{2 0 0}$ laser-driven hohlraum shots have been loaded into this system. The data base covers both empty and fuel-ball-containing Cairn designs as well as several other geometries.

Scaling parameters for which data exist span the following ranges:

- Incident laser energy $\left(\mathrm{E}_{\mathrm{L}}\right): 10 \mathrm{~J} \leq \mathrm{E}_{\mathrm{L}} \leq$ $10^{4} \mathrm{~J}$.

- Laser wavelength $(\lambda): 10.6,1.06,0.53$, and $0.35 \mu \mathrm{m}$.

- Laser-pulse duration ( $\tau$ ): $0.1 \mathrm{~ns} \leq \tau \leq$ $6 \mathrm{~ns}$.

- Hohlraum size $(\mathrm{V}): 7<10^{-6} \mathrm{~cm}^{3} \leq \mathrm{V} \leq$ $0.012 \mathrm{~cm}^{3}$.

Figure 4-1 illustrates typical Cairn hohlraum characteristics. The Shiva or Argus laser beams were focused into the entrance apertures, allowing at least $100 \mu \mathrm{m}$ between marginal rays and the lip of the entrance hole. To maintain calculable geometries, axial symmetry was preserved, except for the diagnostic holes required by the "Dante" soft x-ray spectrometers. Table 4-1 lists the irradiation conditions to which these targets were exposed. Specific alignment geometries have not changed from those reported last year. ${ }^{2}$ Many measurements were made during each target irradiation, and these measurements were principally intended to elucidate the three fundamental processes: coupling of the target to the laser pulse, soft $x$-ray drive, and suprathermal electron preheat. Very few data have been accumulated so far on drive uniformity at the capsule, preheating of the implosion assembly, or stability.

Table 4-2 lists all of the empty-hohlraum target irradiations carried out to date.

Absorption Studies. Figure 4-2 includes all absorption measurements made since late 1978 on empty Caim hohlraums and reveals that the average absorption has been slightly over $80 \%$. Absorption in this context means the fraction of the incident laser energy not accounted for by reflected beam diagnostics (RBD). In fact, more than one measurement system provides absorption information. Besides indirect measures, there are three groups of detectors inside the target chamber specifically designed to

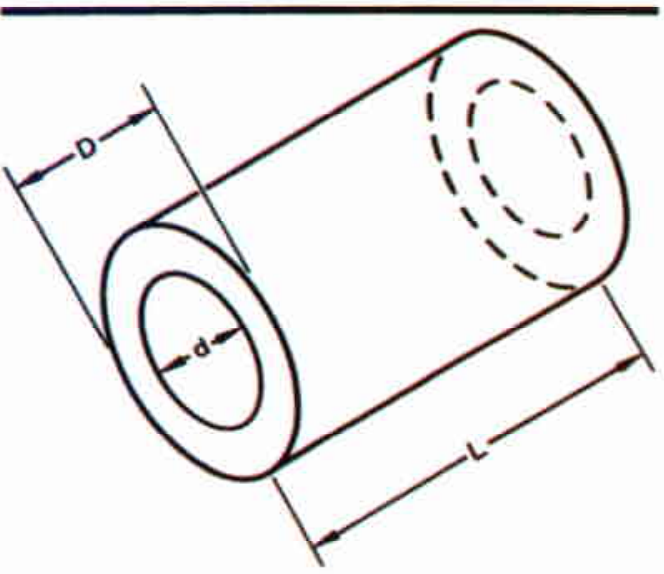

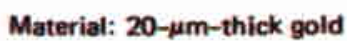

Typical dimensions

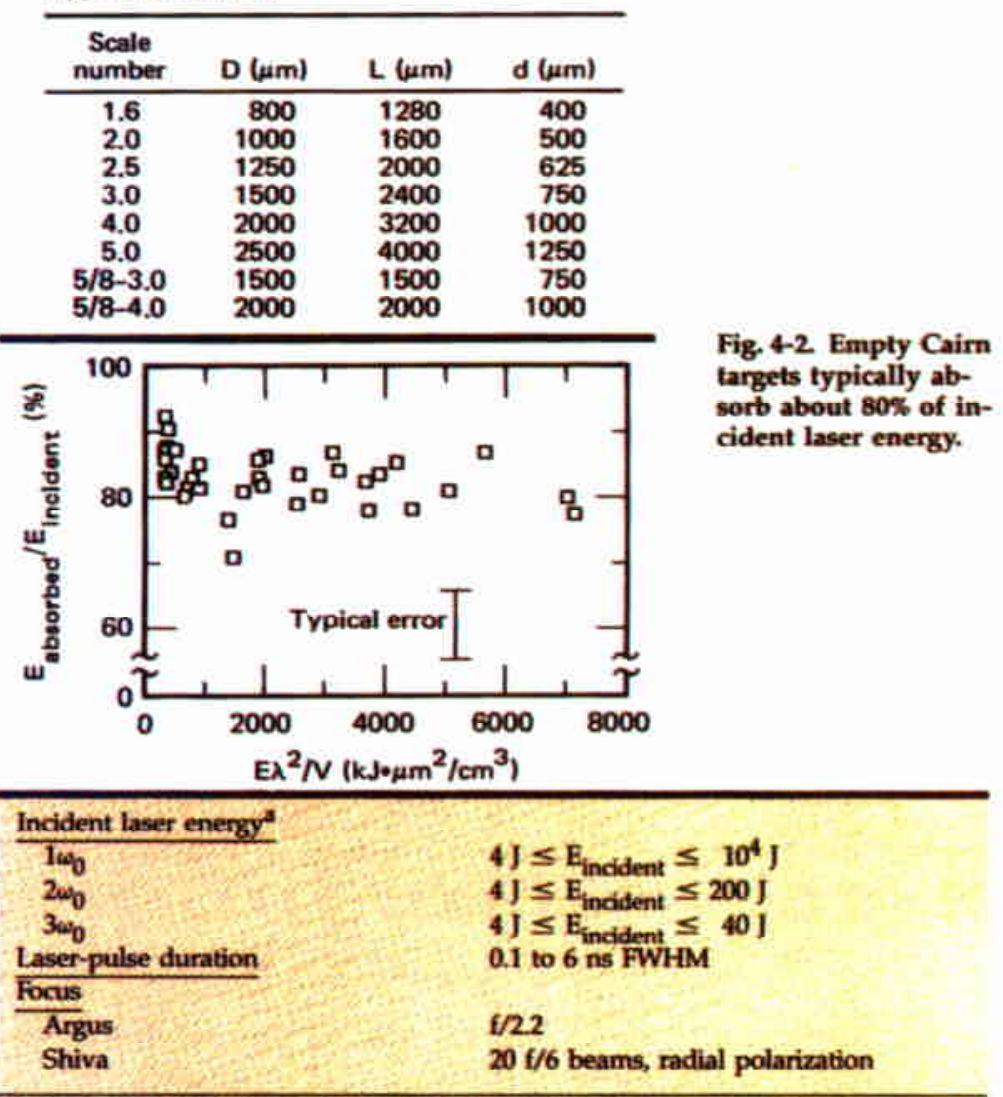

"Minimum energy determined by diagnostics limitations; maximum energy determined by damage threshold.
Fig. 4-1. Hohlraums are gold cylinders with characteristics listed here. sorb about $80 \%$ of incident laser energy.

Table 4-1. Irradiation conditions at $1 \omega_{0}$ $2 \omega_{0}$ and $3 \omega_{0}$ 
However, none of the data in Fig. 4-7 has been so corrected.

Suprathermal X-Ray Scaling. The suprathermal $x$-ray flix has been monitored as an indication of suprathermal electron population, and a variety of scaling relations have been devised to explain its behavior. Table 4-3 contains $\mathbf{x}$-ray flux measurements from channels centered between 25 and $350 \mathrm{keV}$ for all empty hohlraum targets in our data base. The units employed are $10^{12} \mathrm{keV} / \mathrm{keV}$ and as the tabulation shows the hohlraum data cover seven orders of magnitude. Besides the obvious technical challenge of absolute measurements over such a range, these data present a severe test for any scaling hypotheses.

Last year we observed that the suprathermal $x$-ray flux at a given $x$-ray energy from hohlraum targets, when normalized by the absorbed laser energy, scaled as E/V,

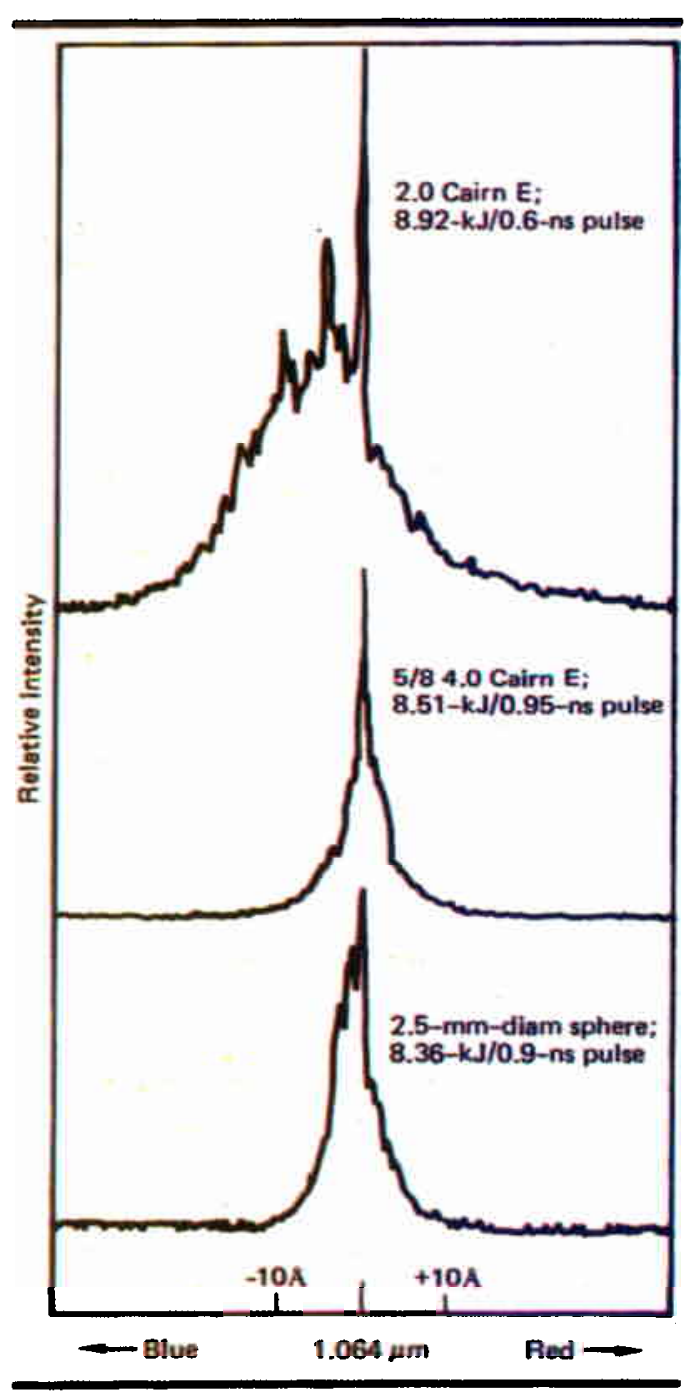

where $\mathrm{E}$ is the laser energy and $\mathrm{V}$ is the hohlraum volume (e.g., see Fig. 5-81, Ref. 2). This year new data at $\lambda=0.35^{\circ} \mu \mathrm{m}$ and $\lambda$ $=0.53 \mu \mathrm{m}$ were added that caused us to change our scaling parameter to $\mathrm{E}^{2} / \mathrm{V}$. Figure 4-8(a) through (e) plots the normalized suprathermal $x$-ray fluxes at different $x$-ray energies in units of $(\mathrm{keV})^{-1}$ for the empty hohlraum targets in Table 4-3 vs $E \lambda^{2} / V$ in units of $\mathrm{kJ}(\mu \mathrm{m})^{2} / \mathrm{cm}^{3}$. These plots reveal the same trends noted previously, i.e., in spite of lange scatter, the $x$-ray flux detected in any of the listed channels clearly increases as $\mathrm{E} \lambda^{2} / \mathrm{V}$ increases. In all cases, the filter-fluorescer spectrometers measured flux produced predominantly within the hohlraum as bremsstrahlung radiation. The $25-\mathrm{keV}$ emission was certainly
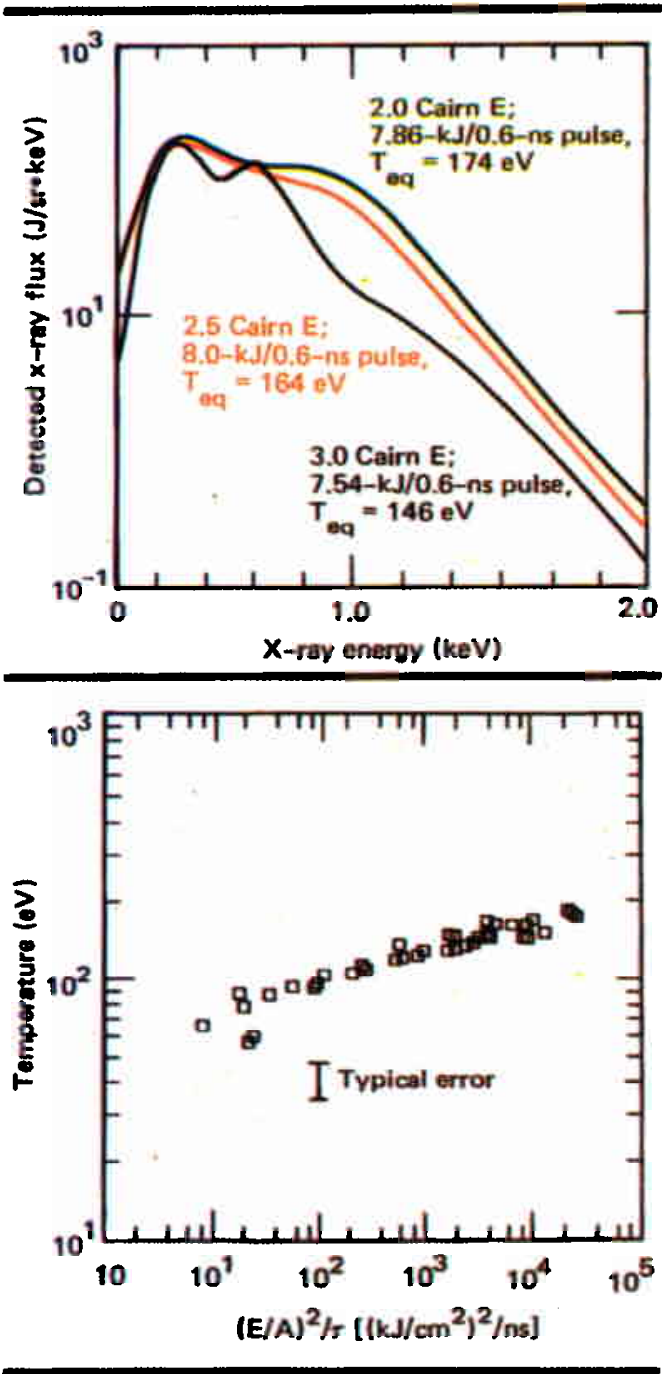

Fig 4-5. Representative backscattered light spectra from hohiraum targets.
Fig 4-6. Representative Dante spectra from 600 ps Cairn irradiations show softening of spectra $a$ hohiraum size is increased.

Fig-47. Equivalent soft $x$ ray brightens temperature scales with Marshak parameter $\mathrm{E} / \mathrm{A} \tau^{1 / 2}$. 


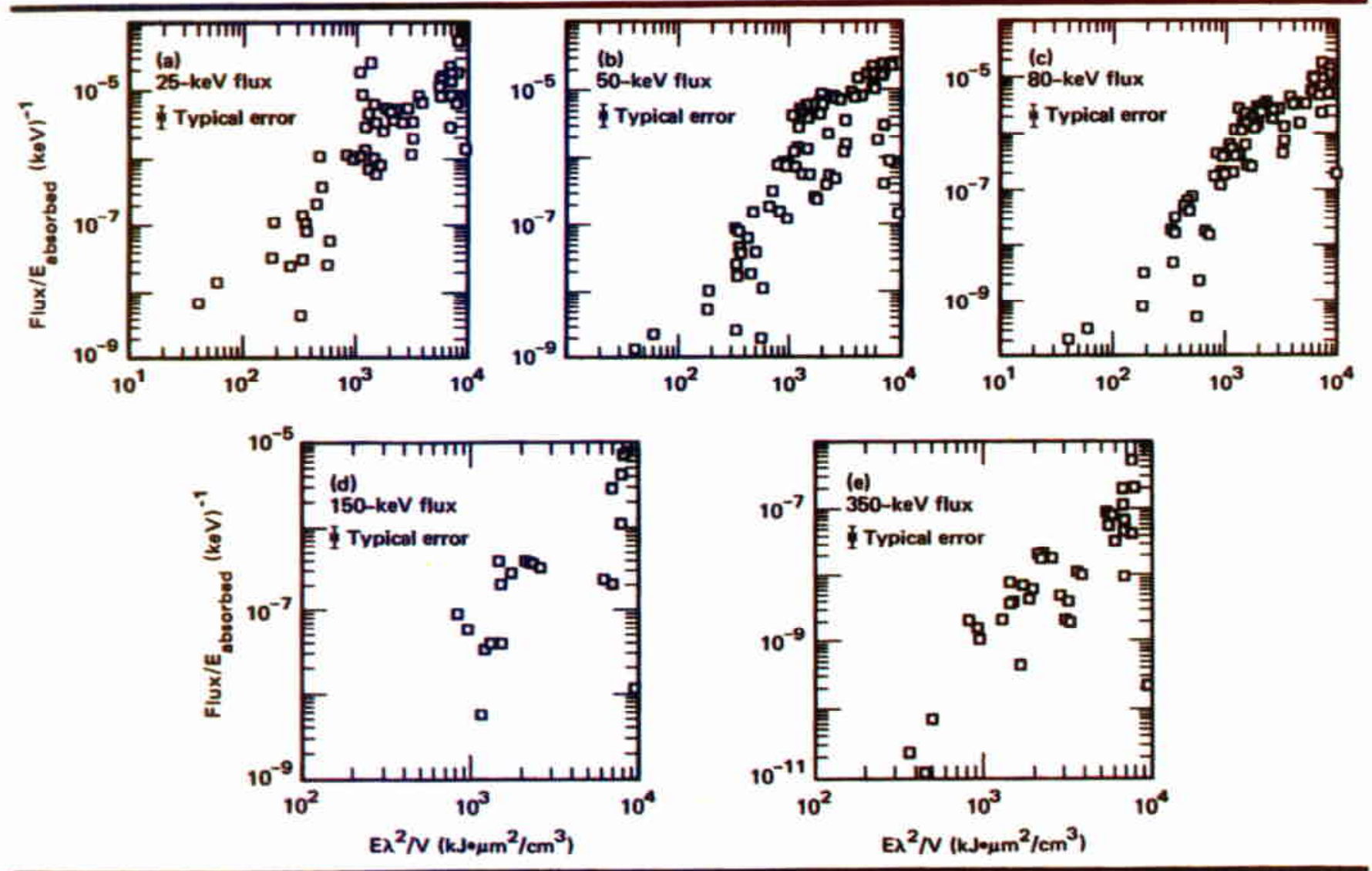

Fig 4-8. Normalized $x$-ray flux scales with $\mathrm{EN}^{2} / \mathrm{V}$. severely attenuated by the 15-to-20- $\mu \mathrm{m}$ thick target walls. The $50-\mathrm{keV} \times$-ray flux was much less affected, and all higher channels were essentially unaffected.

Each individual spectrum shows a compound exponential decline at high energy. This observation led to an attempt to characterize the observed spectra by two suprathermal temperatures. Figure 4-9 shows a number of these $x$-ray spectrum slope (or temperature) estimates drawn from empty hohlraum irradiations at LLNL and LASL ${ }^{3}$ This slope, $\theta_{\mathrm{H}}$ is the lower of the two temperatures that seem to characterize our data. The slope apparently increases with the average laser intensity on the interior of the hohlraum (E/Ar) and thus is found to scale with the parameter $\left(\mathrm{EN}^{2} / \mathrm{Ar}\right)$ as indicated in Fig. 4-9. In the expression for this parameter, $\mathrm{E}$ is the laser energy, $\lambda$ is the wavelength, $A$ is the hohlraum area, and $\tau$ is the pulse length. Such $\mathrm{D}^{2}$ intensity scalings have often been noticed in disk target studies; however, the temperatures observed here are much langer. Simulation calculations ${ }^{4}$ for the $2 \omega_{\text {pe }}$ instability have shown the same onethird-power law suggested by these data.
Figure 4-10(a) through (f) shows exemplary spectra drawn from our data base for a variety of conditions of $E, \lambda, \tau$, and $A$. It is immediately apparent that most of these spectra cannot be adequately described by a single exponential. Empirically, we can obtain a much better fit if we invoke a second, higher "superhot" temperature. This superhot tail is fitted by a temperature $\theta_{\mathrm{SH}}$ that seems to be two to three times larger than $\theta_{\mathrm{H}}$ and scales identically with the parameter $\left(\mathrm{EX}^{2} / \mathrm{A} \tau\right)$.

The suprathermal $x$-ray flux emitted from the target can be described by an approximate thick-target bremsstrahlung relation

Flux $(\epsilon) \cong$ const. $f_{H} E \exp \left(1-\frac{\epsilon}{\theta}\right)$,

where $E$ is the laser energy absorbed by the target, $\mathrm{f}_{\mathrm{H}}$ is the fraction of the absorbed energy appearing in suprathermal electrons, and $\theta$ is a temperature describing the $\mathrm{x}$-ray spectrum. Armed with Eq. (1) and the simple models for $\theta_{\mathrm{H}}$ and $\boldsymbol{\theta}_{\mathrm{SH}}$ just discussed, we need only a means of estimating the fraction of energy in the suprathermal electron population $\mathrm{f}_{\mathrm{H}}$ to predict a thick-target bremsstrahlung spectrum for our targets 
that we can then compare with the experimental data. Several scalings have been proposed, and two are plotted in Fig. 4-10. The curve labeled $L$ is based on a hohlraum-filling plasma model, described in Section $\mathbf{2}$ of this report and Ref. 5 and is given by Eq. (2)

$$
\begin{aligned}
\text { Flux }(\epsilon)= & 2.5 \times 10^{14}\left(\frac{Z}{79}\right) \mathrm{E} \\
& \exp (-\gamma)\left[\exp \left(1-\frac{\epsilon}{\theta_{\mathrm{H}}}\right)\right. \\
& \left.+\frac{1}{25} \exp \left(1-\frac{\epsilon}{\theta_{\mathrm{SH}}}\right)\right] .
\end{aligned}
$$

where

$$
\begin{aligned}
f_{\mathrm{H}} & =0.5 \exp (-\gamma), \\
\gamma & =667\left(\mathrm{~A}^{2} / \lambda^{2} \mathrm{E} \tau\right), \\
\theta_{\mathrm{H}} & =8.618\left(\mathrm{E} \lambda^{2} / \mathrm{A} \tau\right)^{1 / 3}, \\
\theta_{\mathrm{SH}} & =2.675 \theta_{\mathrm{H}} .
\end{aligned}
$$

The curve labeled $M$ is an empirical fit to the $x$-ray data given by

$$
\begin{aligned}
\operatorname{Flux}(\epsilon)= & 2.5 \times 10^{14}\left(\frac{Z}{79}\right) \\
& \mathrm{Ef}_{\mathrm{H}}\left[\exp \left(1-\frac{\epsilon}{\theta_{\mathrm{H}}}\right)\right. \\
& \left.+0.03 \exp \left(1-\frac{\epsilon}{\theta_{\mathrm{SH}}}\right)\right],
\end{aligned}
$$

where

$$
\begin{aligned}
\mathrm{f}_{\mathrm{H}} & =\left[1+\left(7 \times 10^{6}\right)\left(\mathrm{V} / \mathrm{E}^{2}\right)^{2}\right]^{-1}, \\
\theta_{\mathrm{H}} & =8\left(\mathrm{E}^{2} / \mathrm{Ar}\right)^{1 / 3} \mathrm{keV}, \\
\theta_{\mathrm{SH}} & =2.5 \theta_{\mathrm{H}} .
\end{aligned}
$$

In both cases the units of flux $(\epsilon)$ are $\mathrm{keV} / \mathrm{keV}$. As Fig. 4-10 shows, the L scaling tracks the long-pulse-duration experiments well, particularly at high $x$-ray energies. The two scalings do equally well for 600 -ps

Fig. 4-9. Suprathermal temperature $\theta_{H}$ scales with average intensity, $\mathrm{E} / \mathrm{Ar}$, times $\lambda^{2}$.

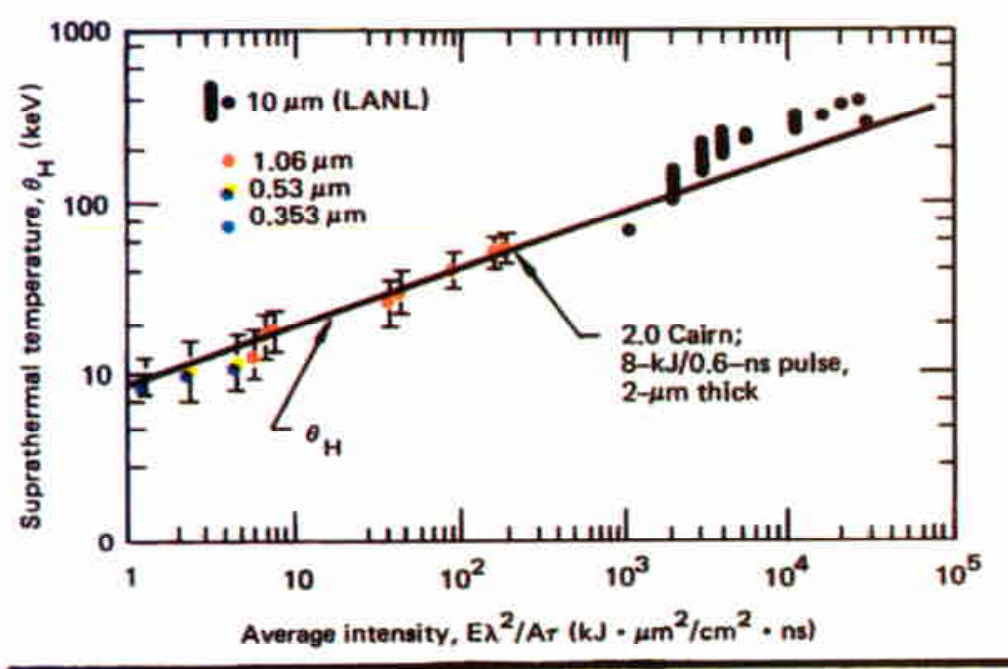

Fig. 4-10. Representative suprathermal x-ray spectra compared to scaling models.

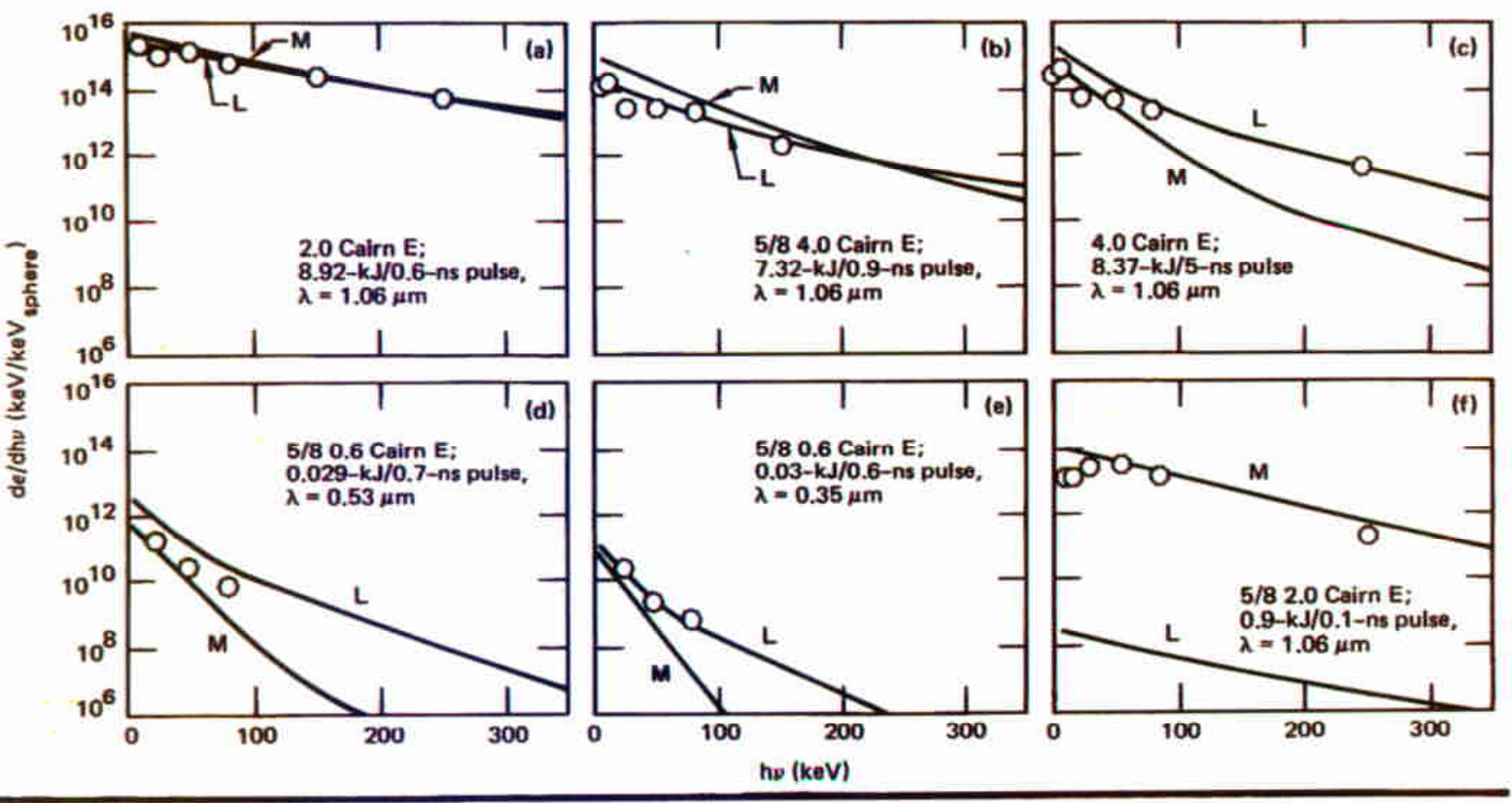


irraditions, but the $\mathrm{L}$ scaling does not agree with the short-pulse tanget experiments. The $\mathrm{M}$ scaling is least reliable for long-pulse experiments, but improves for shorter-pulse experiments.

The primary difference between the two scaling laws is the manner in which they treat the fraction of energy in suprathermal electrons. For L scaling, which is based on a filling model, the fraction [represented as $0.5 \exp (-\delta)]$ depends on laser pulse duration whereas the empirical M prescription scales this fraction (represented as $\mathrm{f}_{\mathrm{H}}$ ) with $\mathrm{E \lambda}^{2} / \mathrm{V}$ only.

Estimates for $\mathbf{f}_{\text {hor }}$ Given an accurate $e x-$ pression for $\theta_{\mathrm{H}}$ calculable from target variables such as hohlraum dimensions, energy on target, pulse duration, etc., the measured $x$-ray flux in any given energy channel can be used to calculate a fraction of energy in hot electrons, $f_{\text {hot }}$ observed on the shot. The 80 -keV flux measurements made on empty hohlraum irradiations were used to estimate $\mathrm{f}_{\text {hot }}$ using both of the models mentioned above. The results are plotted in Fig. 4-11(a) and (b). In both cases the calculated $\mathrm{f}_{\text {hot }}$ of the scaling model is also plotted to show that it traverses the data. These plots include all empty-hohlraum data. The scatter in Fig. 4-11 was reduced by removing the data from hohlraums having questionable focusing or for cases in which the first-bounce corrections to hohlraum filling dominates the spectrum.

The Raman-scattering instability has been identified as one potential source of suprathermal electrons. We have monitored the Raman-shifted scatter from Shiva hohlraum targets using a variety of instrument packages whose absolute calibration is uncertain. Figure 4-12(a) and (b) shows currently available data vs $V / E \lambda^{2}$ and $A^{2} / E \lambda^{2} r$. Once again, the scaling estimates for $f_{\text {hot }}$ are plotted for comparison. These data cannot establish a firm quantitative link between suprathermal electron production and detected Raman scattered light. However, they support the assertion that Raman scattering scales approximately as $f_{\text {hor }}$

Cairn Targets with Capsules. Compression experiments employing several capsule designs and using Caim geometries continued in 1980. Section 5 of Ref. 2 reviews the density measurements performed through 1979 and Table 5-1 of that report summarizes Cairn target experiments performed in 1979. Table 4-4 in this report lists the shot number, scale size, target type, capsule
Fig. 4-11. Experimental estimates for fraction of absorbed energy in hot electrons are compared to model calculations.

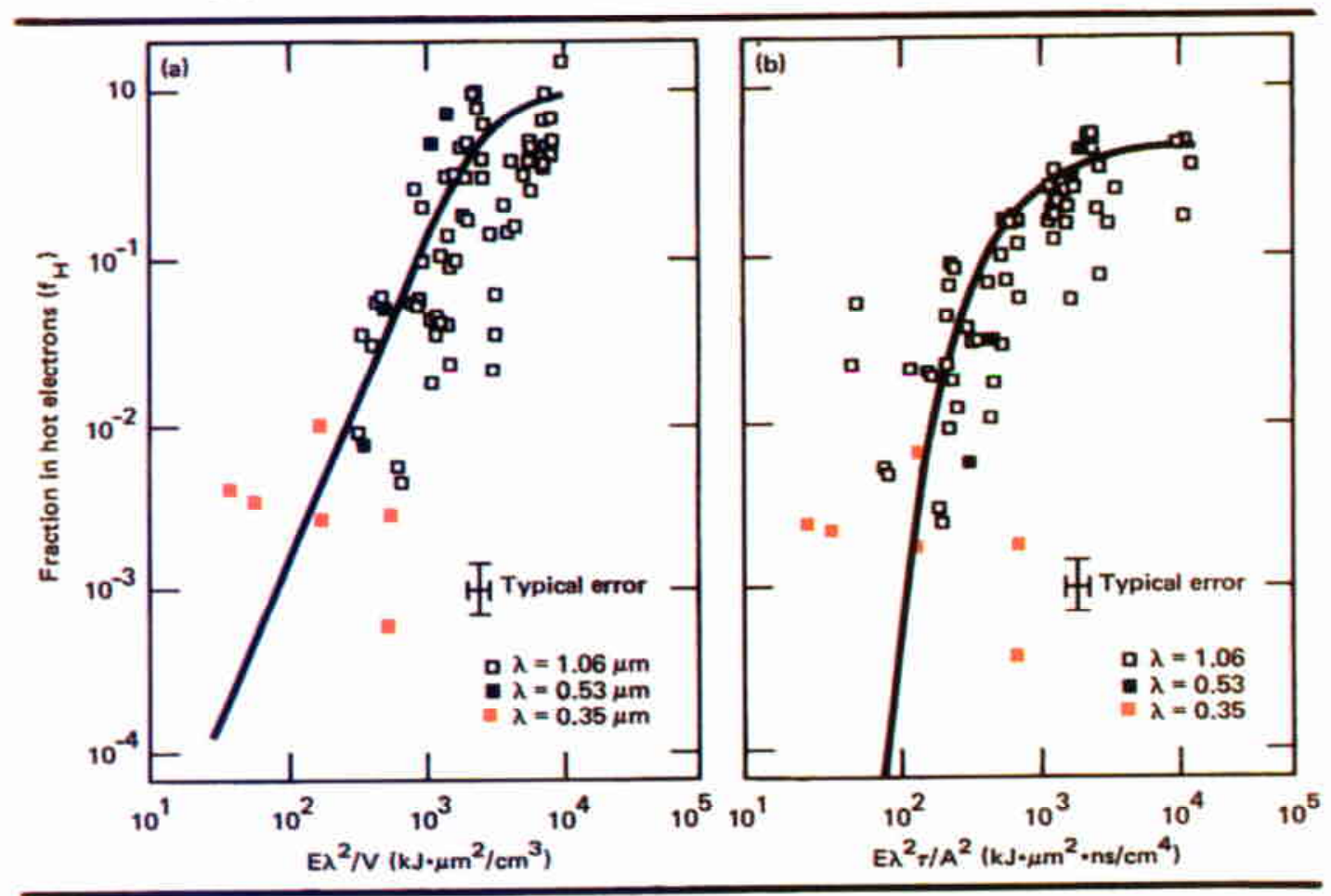




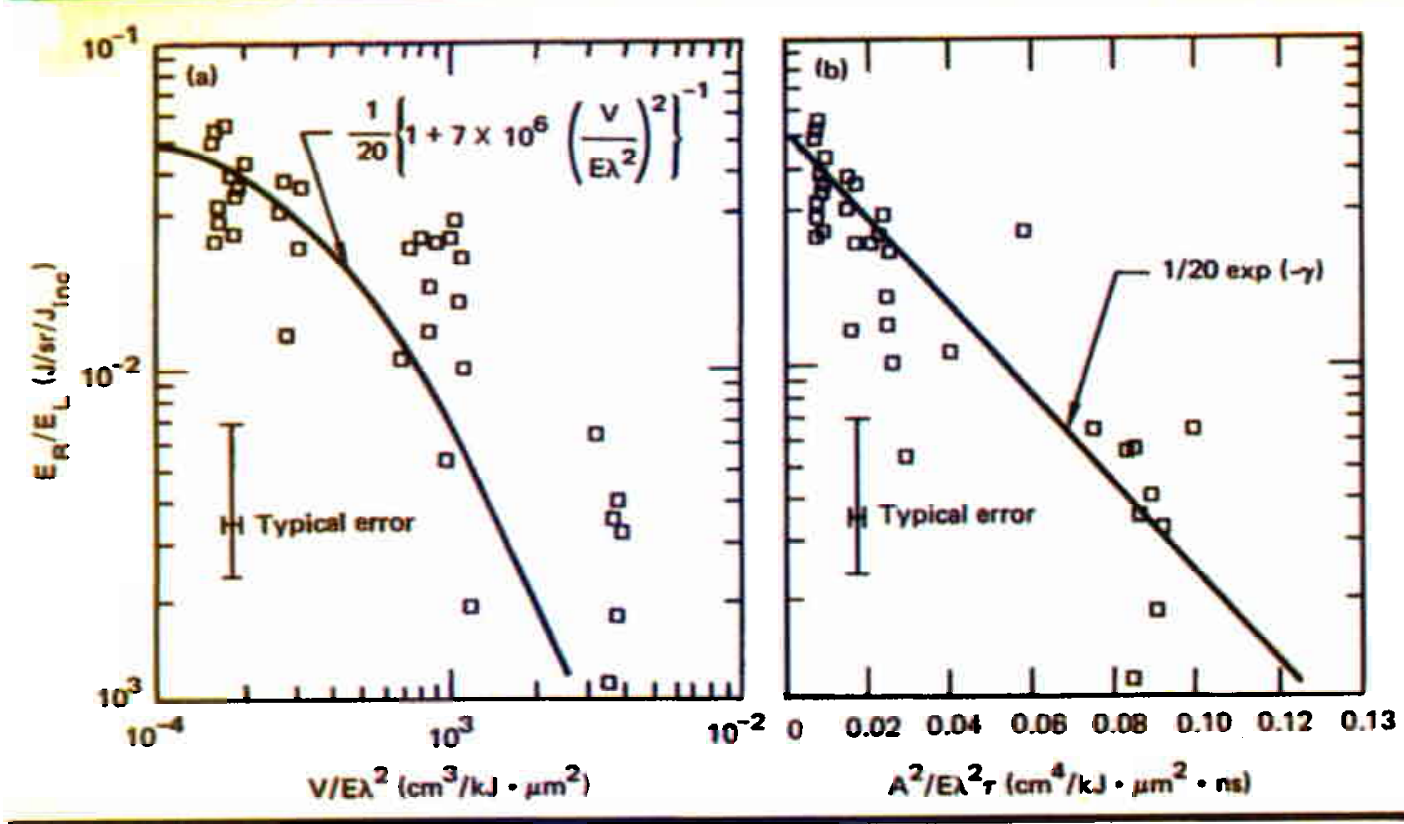

Fig. 4-12. Experimental measurements of normalized Raman scattered energy scale approximately as $\mathrm{f}_{\text {hot }}$

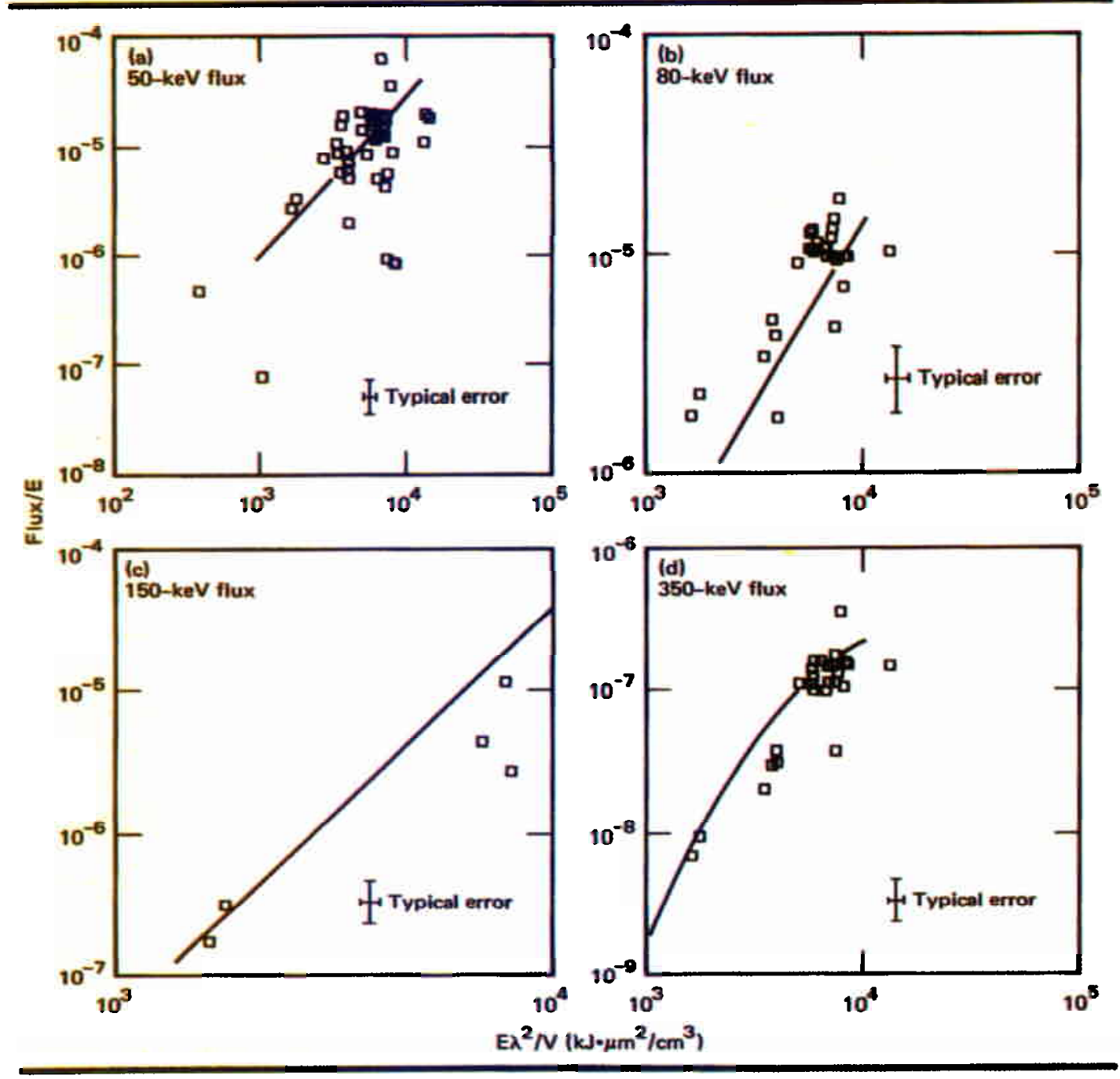

Fig. 4-13. Cairn scaling study. 
Fig 4-14. Cairn scaling study.

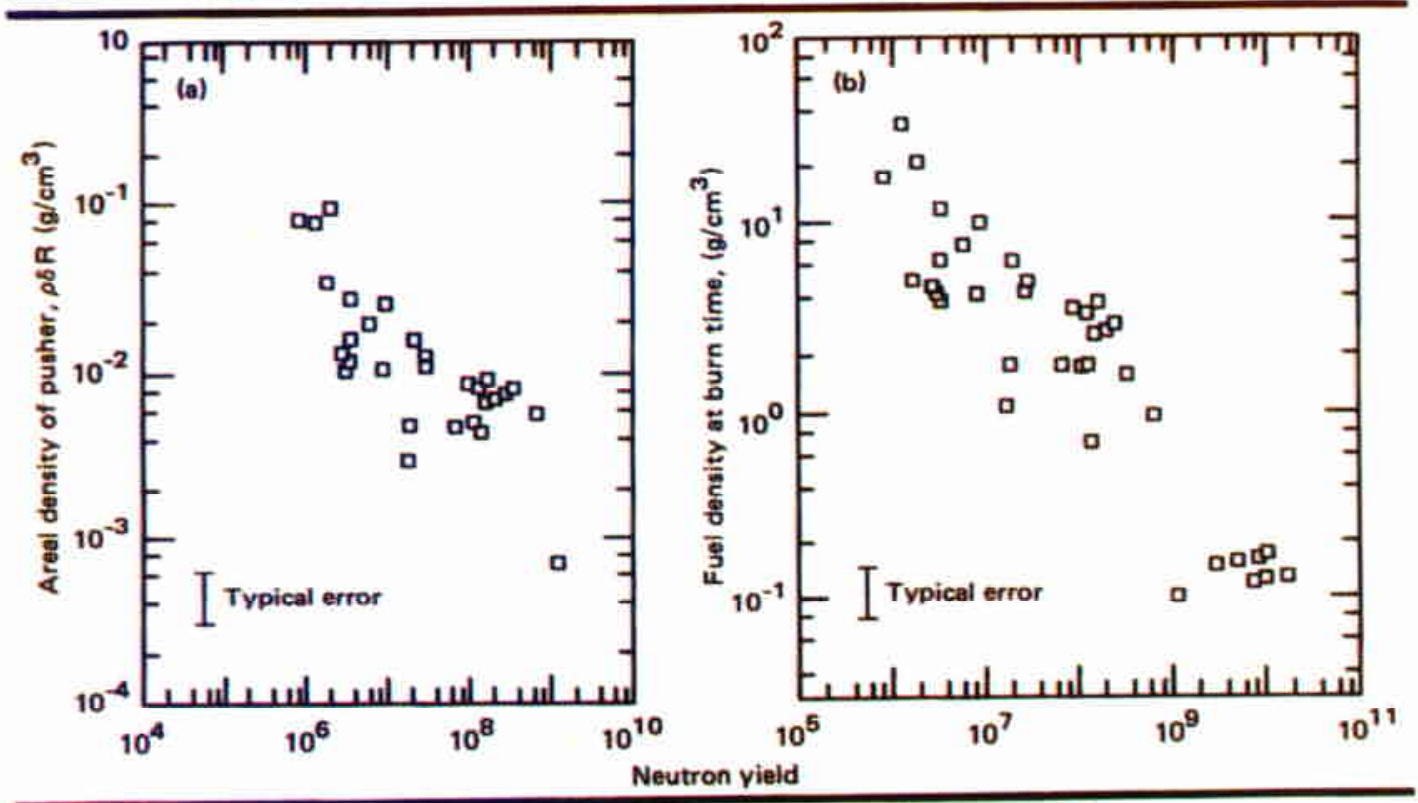

inside diameter in $\mu \mathrm{m}$, capsule wall thickness in $\mu \mathrm{m}$, ablator thickness in $\mu \mathrm{m}$, ablator density in $\mathrm{g} / \mathrm{cm}^{3}$, energy on target in $\mathrm{kJ}$, and pulse duration in ns. Table 4-5 adds equivalent drive temperature measurements in $\mathrm{eV}$ where available and 50 and $80 \mathrm{keV}$ suprathermal $x$-ray fluxes in units of $10^{12}$ $\mathrm{keV} / \mathrm{keV}$. Finally, Table 4-6 updates the list of neutron yields in millions, pusher areal densities in $\mathrm{g} / \mathrm{cm}^{2}$, and LASNEX-inferred burn-time densities in $\mathrm{g} / \mathrm{cm}^{3}$. A fractional error is shown for each measurement. Thus Tables 4-4 to 4-6 expand and update Table 5-1 in Ref. 2 and present information accumulated during 1980.

The additional information accumulated during the last year is plotted in Fig. 4-13. The general trend observed in empty-Cairn scaling studies is again observed, i.e., the flux increases with $\mathrm{E}^{2} / \mathrm{V}$. A line drawn on each plot identifies the mean value of corresponding empty-Caim data. Scattering of the data has increased, but no simple trends emerge.

In the same way, density information is presented in Fig. 4-14(a) through (d), which updates Figs. 5-18 and 5-29 of Ref. 2. Figure 4-14(a) plots pusher areal densities in $\mathrm{g} / \mathrm{cm}^{2}$ vs observed neutron yields. Figure 4-14(b) uses these data together with imaging data to infer burn-time densities in $\mathrm{g} / \mathrm{cm}^{3}$ for all Shiva implosions. Higher density is achieved at the expense of ion temperature and thus neutron yield. In fact, the data in Fig. 4-14(b) fall on or below the line described by $\rho^{2} \mathrm{~N} \simeq 10^{9}$.

Authors: K. R. Manes and J. D. Lindl 


\begin{tabular}{|c|c|c|c|c|c|c|c|}
\hline $\begin{array}{c}\text { Shot } \\
\text { number" }\end{array}$ & $\begin{array}{c}\text { Target } \\
\text { size }\end{array}$ & $\begin{array}{l}\text { Target } \\
\text { shape }\end{array}$ & $\begin{array}{l}\text { Target } \\
\text { type }\end{array}$ & $\begin{array}{l}\text { Wave- } \\
\text { length } \\
(\mu \mathrm{m})\end{array}$ & $\begin{array}{l}\text { Equivalent } \\
\text { temperature } \\
(\mathrm{eV})\end{array}$ & $\begin{array}{c}\text { Incident: } \\
\text { energy } \\
\text { (kJ) }\end{array}$ & $\begin{array}{l}\text { Pulse } \\
\text { duration } \\
\text { (ns) }\end{array}$ \\
\hline 30082017 & $5 / 8$ & 0.60 & Caim E & 0.532 & & 0.023 & 0.70 \\
\hline 30082106 & $5 / 8$ & 0.40 & Caim E & 0.532 & & 0.029 & 0.68 \\
\hline 30082211 & $5 / 8$ & 0.40 & Caim E & 0.532 & & 0.005 & 0.71 \\
\hline 30082615 & $5 / 8$ & 0.40 & Caim E & 0.532 & & 0.026 & 0.79 \\
\hline 30082621 & $5 / 8$ & 0.60 & Caim E & 0.532 & & 0.031 & 0.70 \\
\hline 30082810 & $5 / 8$ & 0.40 & Cairn E & 0.532 & & 0.029 & 0.74 \\
\hline 30111318 & $5 / 8$ & 0.60 & Caim E & 0.353 & & 0.030 & 0.60 \\
\hline 30111407 & $5 / 8$ & 0.40 & Caim E & 0.353 & & 0.031 & 0.60 \\
\hline 30111915 & $5 / 8$ & 0.60 & Caim E & 0.353 & & 0.029 & 0.60 \\
\hline 30112011 & $5 / 8$ & 0.40 & Caim E & 0.353 & & 0.028 & 0.60 \\
\hline 30121126 & $5 / 8$ & 0.80 & Cairn E & 0,353 & 660 & 0.023 & 0.70 \\
\hline 30121206 & $5 / 8$ & 0.80 & Caim E & 0.353 & 88.0 & 0.034 & 0.70 \\
\hline 80011003 & & 200 & Caim E & 1.060 & 77.0 & 0.420 & 2.00 \\
\hline 80011106 & & 2.00 & Caim E & 1.060 & 86.0 & 0.550 & 200 \\
\hline 80011412 & & 2.00 & Caim E & 1.060 & 1350 & 4.990 & 200 \\
\hline 80011419 & & 2.00 & Caim E & 1.060 & 133.0 & 4.690 & 2.00 \\
\hline 80011503 & & 2.00 & Caim E & 1.060 & 143.0 & 4.170 & 200 \\
\hline 80011605 & & 2.00 & Caim E & 1.060 & 133.0 & 2200 & 200 \\
\hline 80011704 & & 3.00 & Caim E & 1.060 & 105.0 & 3.010 & 2.00 \\
\hline 80030505 & & 3.00 & Caim E & 1.060 & 143.0 & 7540 & 0.60 \\
\hline 80030605 & $5 / 8$ & 4.00 & Caim E & 1.060 & 141.0 & 8.190 & 0.60 \\
\hline 80031010 & $5 / 8$ & 3.00 & Caim E & 1.060 & 144.0 & 7.690 & 0.60 \\
\hline 80031103 & & 2.50 & Caim E & 1.060 & 158.0 & 6.350 & 0.60 \\
\hline 80031204 & & 2.50 & Caim E & 1.060 & 164.0 & 8.000 & 0.60 \\
\hline 80031406 & $5 / 8$ & 4.00 & Caim E & 1060 & 143.0 & 6.130 & 0.60 \\
\hline 80031704 & & 2.00 & Caim E & 1.060 & 174.0 & 7.860 & 0.60 \\
\hline 80031903 & $5 / 8$ & 3.00 & Caim $\mathbf{E}$ & 1.060 & 147.0 & 9.220 & 0.60 \\
\hline 80032006 & & 2.00 & Caim E & 1.060 & 173.0 & 8.000 & 0.60 \\
\hline 80032502 & & 3.00 & Caim E & 1.060 & 147.0 & 7.120 & 0.60 \\
\hline 80040802 & $5 / 8$ & 3.00 & Caim E & 1.060 & 1440 & 7.370 & 0.60 \\
\hline 80040805 & $5 / 8$ & 3.00 & Caim E & 1060 & 159.0 & 7.610 & 0.60 \\
\hline 80051908 & $1 / 2$ & 2.00 & Caim & 1.060 & & 4.100 & 0.60 \\
\hline 80052007 & $1 / 2$ & 2.00 & Caim & 1.060 & & 4.000 & 0.60 \\
\hline 80052020 & $1 / 2$ & 200 & Caim & 1.060 & & 3900 & 0.60 \\
\hline 80052103 & $1 / 2$ & 2.00 & Caim & 1.060 & & 4000 & 0.60 \\
\hline 80062003 & $1 / 2$ & 2.00 & Caim BL. & 1.060 & & 0.900 & 0.10 \\
\hline 80062007 & $1 / 2$ & 200 & Cairn BL & 1.060 & & 0.850 & 0.10 \\
\hline 80070213 & $1 / 2$ & 2.00 & Caim BL. & 1.060 & & 3.250 & 0.60 \\
\hline 80072104 & & 2.00 & Caim E & 1.060 & & 9.200 & 0.60 \\
\hline 80072202 & & 2.25 & Sphere & 1.060 & & 7.970 & 0.60 \\
\hline 80072204 & & 2.00 & Caim E & 1.060 & & 8.920 & 0.60 \\
\hline 80072304 & & 200 & Caim E & 1.060 & & 7.830 & 0.60 \\
\hline 80073005 & & 2.25 & Sphere & 1.060 & & 7.800 & 0.60 \\
\hline 80092503 & & 2.00 & Heinz B & 1.060 & & 2.960 & 0.54 \\
\hline 80092514 & & 2.00 & Heinz B & 1.060 & & 3.000 & 0.54 \\
\hline 80092606 & & 2.00 & Heinz B & 1.060 & & 3370 & 0.54 \\
\hline 80092903 & & 2.00 & Heinz B & 1.060 & & 1.310 & 0.54 \\
\hline 80092905 & & 2.00 & Heinz B & 1.060 & & 3.500 & 058 \\
\hline 80101408 & & 200 & Heinz B & 1.060 & & 3.450 & 0.60 \\
\hline 80101604 & & 2.00 & Heinz B & 1.060 & & 3.840 & 0.60 \\
\hline
\end{tabular}

\section{4-11a}


Hohlraum Studies

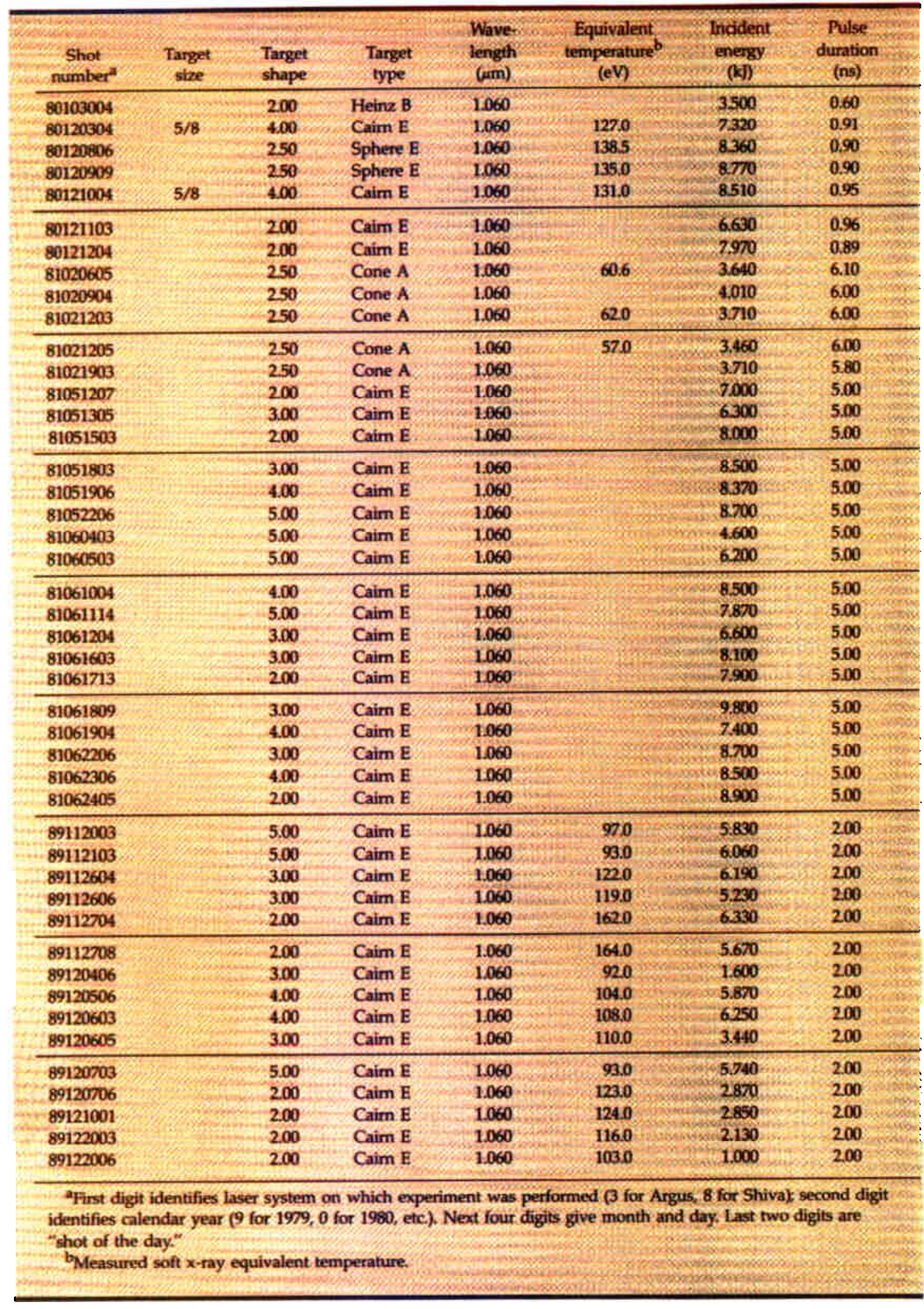

Table 4-2. Hohlraum scaling summary. 
Table 4-3. Empty hohlraum $x$-ray flux sealing date. Units are $10^{12} \mathrm{keV} / \mathrm{keV} \cdot 4 \mathrm{x}$.
Hohlraum Studies

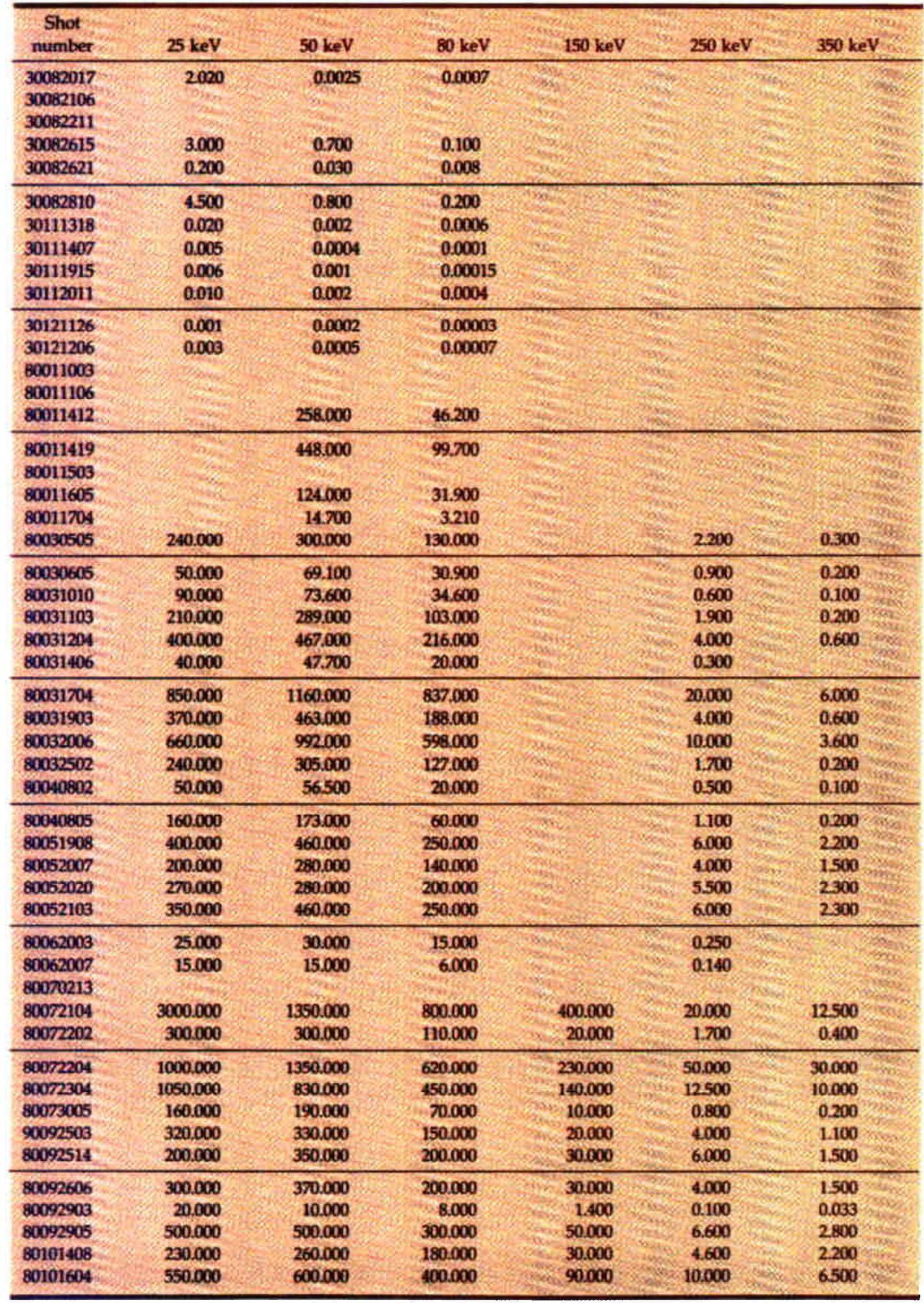


Hohlraum Studies

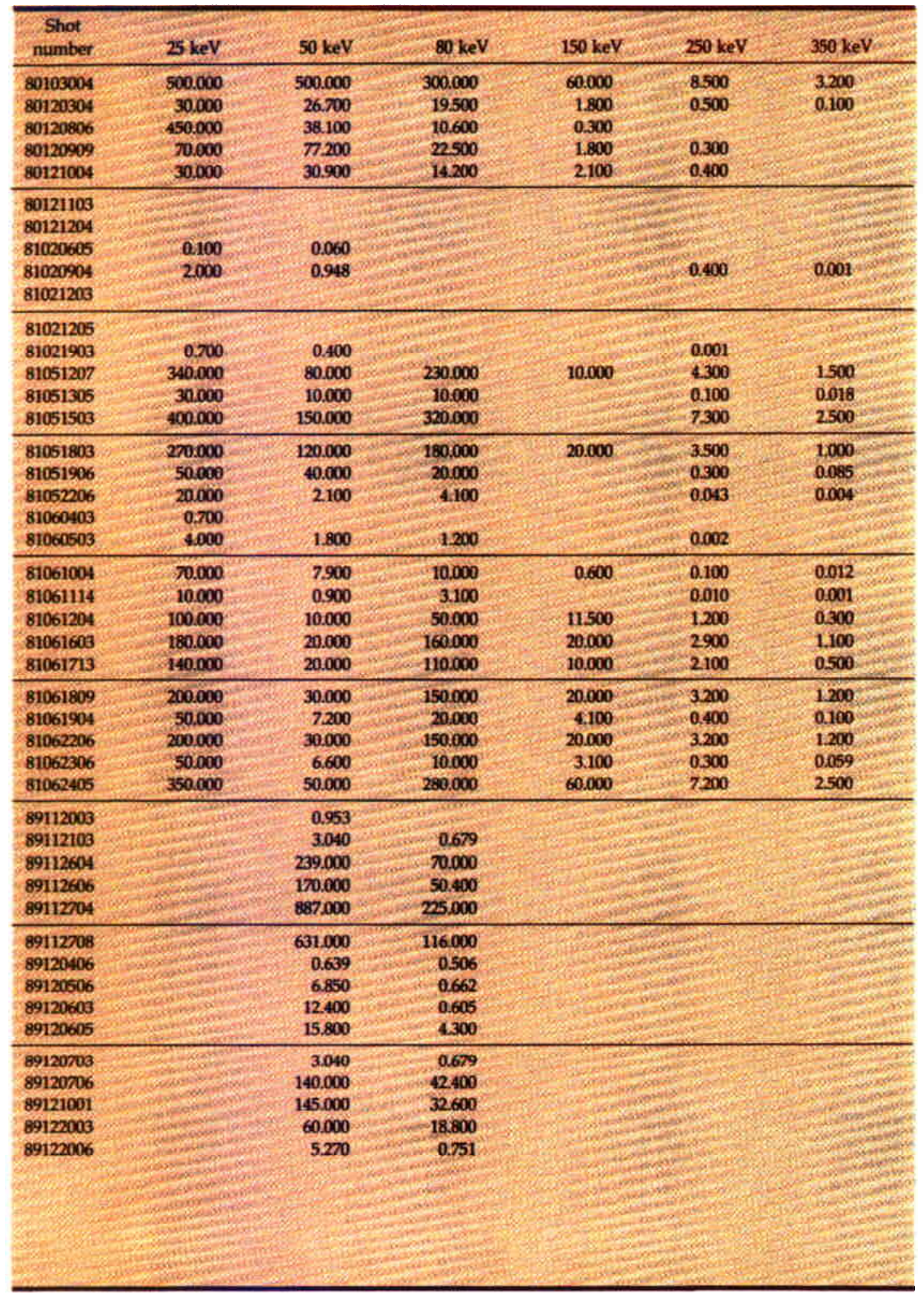

Table 4-3. (cont.) 
Table th Cairn summary.

\section{Hohlraum Studies}

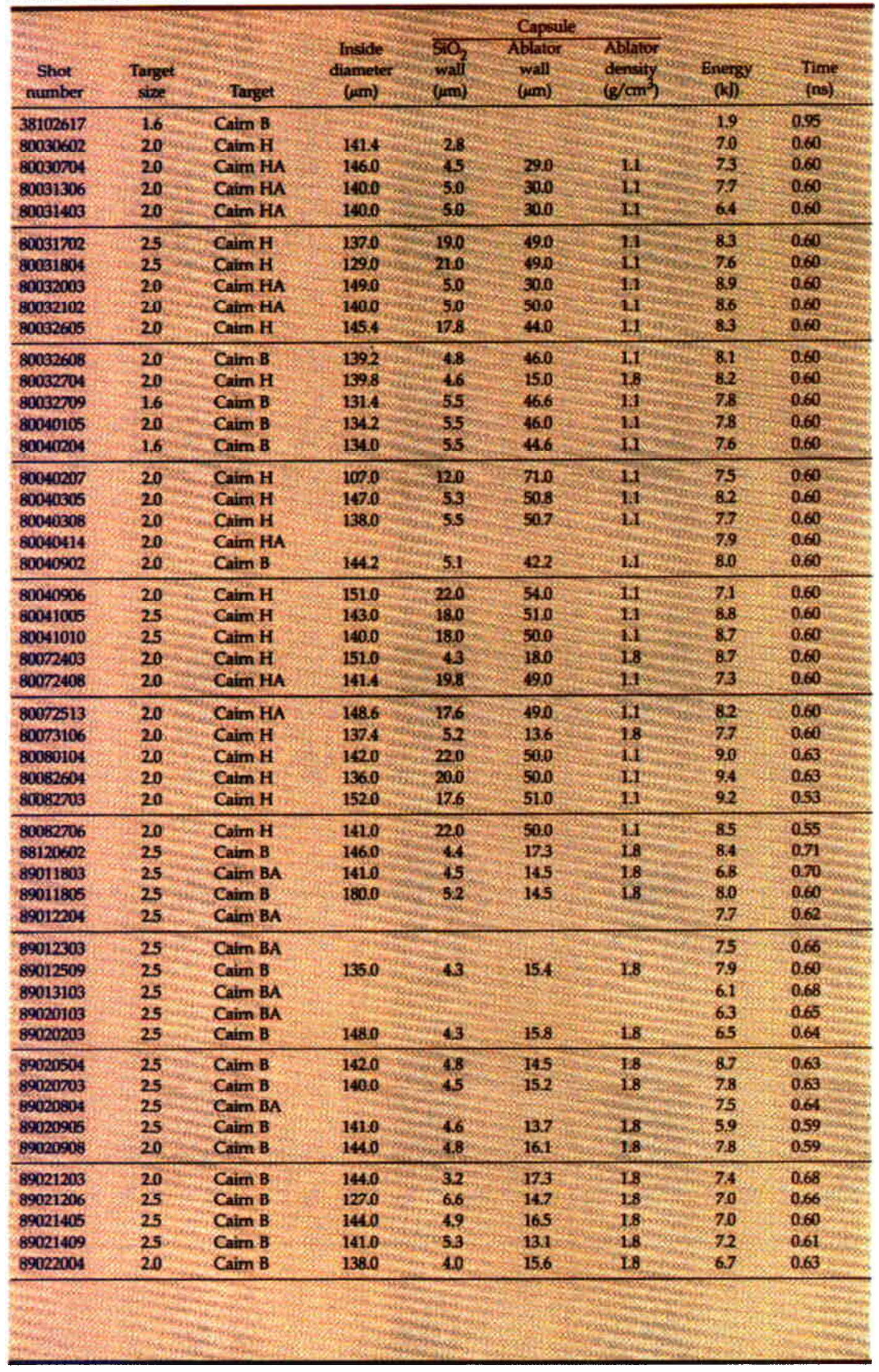




\begin{tabular}{|c|c|c|c|c|c|c|c|c|}
\hline $\begin{array}{l}\text { Shot } \\
\text { number. }\end{array}$ & $\begin{array}{c}\text { Target } \\
\text { size }\end{array}$ & Target & $\begin{array}{c}\text { Inside } \\
\text { diameter } \\
\text { (um) }\end{array}$ & $\begin{array}{l}\mathrm{SiO}_{2} \\
\text { wall } \\
\text { (um) }\end{array}$ & $\begin{array}{l}\text { Ablator } \\
\text { wall } \\
\text { (um) }\end{array}$ & $\begin{array}{l}\text { Ablator } \\
\text { density } \\
\left(\mathrm{g} / \mathrm{cm}^{3}\right)\end{array}$ & $\begin{array}{l}\text { Energy } \\
\text { (k) }\end{array}$ & $\begin{array}{l}\text { Time } \\
\text { (ns) }\end{array}$ \\
\hline 89022006 & 20 & Caim B & 147.0 & 4.6 & 165 & 18 & 7.1 & 0.54 \\
\hline 89022604 & 2.0 & Caim H & 143.0 & 4.6 & 142 & 1.8 & 76 & 0.58 \\
\hline 89022606 & 20 & Caim BA & & & & & 6.6 & 0.60 \\
\hline 89022704 & 2.0 & Caim $\mathrm{H}$ & 141.0 & 4.9 & 17.2 & 1.8 & 73 & 0.63 \\
\hline 89022709 & 20 & Caim BA & & & & & 67 : & 6.25 \\
\hline 89022802 & 25 & Caim B & 134.0 & 4.8 & 17.4 & 1.8 & 8.0 & 0.65 \\
\hline 89022808 & 20 & Caim B & 138.0 & 5.0 & 145 & 1.8 & 80 & 0.59 \\
\hline 89030104 & 2.0 & Caim B & 135.0 & 4.8 & 13.0 & 18 & 13 & 0.70 \\
\hline 89030108 & 20 & Caim B & 135.0 & 5.0 & 13.0 & 1.8 & 7.4 & 0.48 \\
\hline 89030202 & 20 & Caim HA & 138.0 & 5.0 & 13.0 & 1.8 & 75 & 0.59 \\
\hline 89030213 & 25 & Caim B & 141.0 & 42 & 13.7 & 1.8 & 9.1 & 0.62 \\
\hline 89030505 & 20 & Caim HA & & & & & 4.7 & 0.56 \\
\hline 89030509 & 20 & Caim B & 1450 & 4.2 & 130 & 1.8 & 6.0 & 0.61 \\
\hline 89031207 & 25 & Caim B & 150.0 & 4.0 & 163 & 1.8 & 73 & 0.72 \\
\hline 89031211 & 2.0 & Caim B & 144.0 & 45 & 13.0 & 1.8 & 82. & 0.65 \\
\hline 89031304. & 2.0 & Caim H & 143.0 & 5.1 & 11.7 & 1.8 & 7.2 & 0.63 \\
\hline 89031406 & 20 & Caim H & 137.0 & 4.1 & 15.6 & 1.8 & 72 & 0.78 \\
\hline 89031505 & 25 & Caim $\mathrm{H}$ & 140.0 & 5.0 & 126 & $\mathbf{1 . 8}$ & 73 & 0.78 \\
\hline 89031508 & 25 & Caim H & 144.0 & 4.6 & 130 & 1.8 & 7.7 & 0.58 \\
\hline 89031904 & 20 & Cairn $\mathrm{H}$ & 145.0 & 25 & 8.8 & 1.8 & 7.8 & 0.59 \\
\hline 89031907 & 20 & Caim H & 136.0 & 39 & 11.5 & 1.8 & 70 & 0.63 \\
\hline 89032102 & 20 & Caim $\mathrm{H}$ & 145.0 & 4.1 & 11.1 & 1.8 & 7.6 & 0.54 \\
\hline 89032104 & 20 & Caim B & 140.0 & 5.0 & 53.0 & 1.1 & 68 & 0.60 \\
\hline 89032206 & 25 & Caim B & & & & 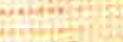 & 8.8 & 0.80 \\
\hline 89032212 & 2.0 & Caim H & 144.0 & 1.8 & 21.0 & 1.8 & 70 & 0.59 \\
\hline 89032305 & 20 & Caim $\mathrm{H}$ & 141.0 & 1.7 & 21.0 & 1.8 & 65 & 0.59 \\
\hline 89032309 & 20 & Caim H & 140.0 & 5.0 & 110.0 & L1 & 6.9 & 0.66 \\
\hline 89082306 & 2.0 & Caim $\mathrm{H}$ & 143.0 & 4.6 & 14.5 & 1.8 & 73 & 0.60 \\
\hline 89083105 & 20 & Caim HA & & & & & 6.3 & 0,60 \\
\hline 89090609 & 2.0 & Cairn $\mathrm{H}$ & 145.0 & 4.4 & 13.0 & 1.8 & 6.8 & 0.58 \\
\hline 89090603 & 20 & Caim I & $144.0^{\circ}$ & 4.0 & 14.1 & 1.8 & 8.0 & 058 \\
\hline 89090605 & 20 & Caim ] & 145.0 & 4.2 & 13.0 & 1.8 & 7.4 & 050 \\
\hline 89090705 & 20 & Caim HA & tin & & & & 7.3 & 0.60 \\
\hline 89090708 & 20 & Cairn HA & & & 60635 & & 79 & 0.59 \\
\hline 89091004 & 20 & Caim I & 144.0 & 45 & 13.9 & 1.8 & 7.4 & 0.60 \\
\hline 89091007 & 20 & Caim $\mathrm{H}$ & 138.0 & 4.3 & 15.6 & 18 & 7.6 & 0.60 \\
\hline 89091302 & 20 & Caim HA & & & & & 6.4 & 0.60 \\
\hline 89091304 & 20 & Caim HA & & & & & 7.2 & 0.60 \\
\hline 89091403 & 20 & Caim I & 147.0 & 4.9 & 122 & 1.8 & 7.3 & 0.60 \\
\hline 89091704 & 20 & Caim I & 143.0 & 6.0 & 14.5 & 1.8 & 7.7 & 0.63 \\
\hline 89091706 & 2.0 & Caim J & 138.0 & 5.1 & 14.8 & 1.8 & 7.0 & 0.63 \\
\hline 89091803 & 2.0 & Caim 1 & 142.0 & 4.7 & 15.0 & 1.8 & 5.6 & 0.63 \\
\hline 89092104 & 20 & Caim HA & & & & & 73 & 058 \\
\hline 89100405 & 1.6 & Caim B. & 138.0 & 4.8 & 48.8 & 1.1 & 8.3 & 0.60 \\
\hline 89100409 & 1.6 & Cairn B & 151.0 & 5.2 & 50.9 & 1.1 & 7.8 & 0.60 \\
\hline 89100503 & 20 & Caim B & 141.0 & 5.0 & 50.0 & 1.1 & 7.9 & 0.60 \\
\hline 89100506 & 20 & Caim B & 159.0 & 55 & 48.6 & 1.1 & 8.1 & 0.60 \\
\hline 89100802. & 3.0 & Caim B & 155.0 & 4.9 & 50.0 & 1.1 & 1.4 & 0.60 \\
\hline 89100804 & 30 & Caim B & 148.0 & 5.3 & 49.7 & 1.1 & 3.9 & 0.60 \\
\hline 89100903 & 20 & Caim $\mathrm{H}$ & 115.0 & 13.0 & 67.0 & 11 & 8.2 & 0.60 \\
\hline 89101105 & 25 & Caim H & & & & & 8.8 & 100 \\
\hline 89101504 & 2.0 & Caim K & & & & & 8.0 & 0.60 \\
\hline 89101603 & 20 & Caim K & 138.0 & 4.6 & 15.2 & 1.8 & 7.0 & 0.60 \\
\hline
\end{tabular}

Table 4-4. (cont) 


\begin{tabular}{|c|c|c|c|c|c|c|c|}
\hline $\begin{array}{c}\text { Shot } \\
\text { number }\end{array}$ & $\begin{array}{c}\text { Temperature } \\
(\mathrm{eV})\end{array}$ & $\begin{array}{c}50 \\
\left(10^{12} \mathrm{keV} / \mathrm{keV}\right)\end{array}$ & $\stackrel{80}{\left(10^{12} \text { keV/keV) }\right.}$ & $\begin{array}{l}\text { Shot } \\
\text { number }\end{array}$ & $\begin{array}{c}\text { Temperature } \\
(\mathrm{eV})\end{array}$ & $\left(10^{12} \mathrm{keV} / \mathrm{keV}\right)$ & $(10$ \\
\hline 38102617 & 134.0 & 110.000 & & 89022006 & & & \\
\hline 80030602 & & 540.000 & 500.000 & 89002604 & & & \\
\hline 80030704 & & & & 89022606 & & & \\
\hline 80031306 & & 560.000 & 500.000 & 89022704 & & & \\
\hline 80031403 & & 520.000 & 380.000 & 89022709 & & 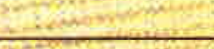 & \\
\hline 80031702 & & 500.000 & 260.000 & 89022808 & 1700 & 1000.000 & \\
\hline 80031804 & & 280.000 & 160.000 & 89022808 & & 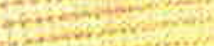 & \\
\hline 80032003 & & 900.000 & 760.000 & 89030104 & & 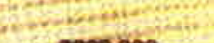 & \\
\hline 80032102 & & 360.000 & & 89030108 & 1850 & 3000.000 & \\
\hline 80032606 & & 300.000 & 240.000 & 89030202 & & 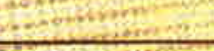 & \\
\hline 80032608 & & 760.000 & 670,000 & 89030213 & 174.0 & & \\
\hline 80032704 & & 980000 & 750.000 & 89030505 & & & \\
\hline 80032709 & & & & 89030509 & 175.0 & 330.000 & \\
\hline 80040105 & & & & 89031207 & 159.0 & 520.000 & \\
\hline 80040204 & & 550.000 & 490.000 & 89031211 & & 2.i...... & \\
\hline 80040207 & & 630.000 & 500.000 & 89031304 & & pooto & \\
\hline 80040305 & & 650.000 & 500.000 & 89031406 & & $\lim _{x \rightarrow \infty}$ & \\
\hline 80040308 & & 640000 & 470.000 & 89031505 & & new1s & \\
\hline 80040414 & & 450.000 & 360.000 & 89031508 & & 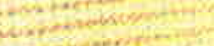 & \\
\hline 80040902 & & 700.000 & 600.000 & 89031904 & & vaxwons & \\
\hline 80040906 & & & & 89031907 & & Hitin & \\
\hline 80041005 & & 290.000 & 100.000 & 89032102 & & Wint..ts & \\
\hline 80041010 & & 350.000 & 230.000 & 89032104 & & Whers & \\
\hline 80072403 & & 2000,000 & 1000.000 & 89032206 & & 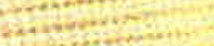 & \\
\hline 80072408 & & 650.000 & 400.000 & 89032212 & & Extitity & \\
\hline 80072513 & & 860.000 & 700.000 & 89032305 & & Menting & \\
\hline 80073106 & & 650.000 & 530.000 & 89032309 & & wotere & \\
\hline 80080104 & & 520.000 & 400.000 & 89082306 & & andinas & \\
\hline 80082604 & & 50.000 & 570.000 & 89083105 & & andintim & \\
\hline 80082703 & & 50.000 & 560.000 & 89090509 & & arents & \\
\hline 80082706 & & 50.000 & 500.000 & 89090603 & & fenting & \\
\hline 88120602 & & & & 89090605 & & a & \\
\hline 89011803 & & & & 89090705 & & & \\
\hline 89011805 & & & & 89090708 & & 4.thente & \\
\hline 89012204 & & & & 89091004 & & 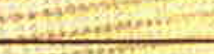 & \\
\hline 89012303 & & & & 89091007 & & arrent... & \\
\hline 89012509 & & & & 89091302 & & $5 x+2$ & \\
\hline 89013103 & & & & 89091304 & & sestontwita & \\
\hline 89020103 & & & & 89091403 & & pritints. & \\
\hline 89020203 & & & & 89091704 & & 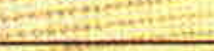 & \\
\hline 89020504 & & 110.000 & & 89091706 & & 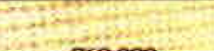 & \\
\hline 89020703 & & 800.000 & & 89091803 & & 760.000 & \\
\hline 89020804 & & & & 89092104 & & & \\
\hline 89020905 & & 300.000 & & 89100405 & 174.0 & 1000,000 & \\
\hline 89020908 & & 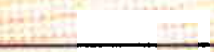 & & 89100409 & 168.0 & $\begin{array}{r}1000.000 \\
\end{array}$ & \\
\hline 89021203 & & & & 89100503 & 177.0 & 1000.000 & \\
\hline 89021206 & & & & 89100506 & 173.0 & 1000000 & \\
\hline 89021405 & & & & 89100802 & 90.0 & 4.300 & \\
\hline 89021409 & & & & 89100804 & 129.0 & 1.900 & \\
\hline 89022004 & & & & 89100903 & & 1000.000 & \\
\hline E. & & & & 89101105 & & 400.000 & \\
\hline$\therefore$ & & & & 89101504 & & 220.000 & \\
\hline$y^{2}$ & & & & 89101603 & & 230.000 & \\
\hline$\varepsilon^{2}$ & & 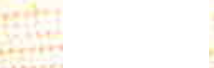 & & & & $\ldots$ & \\
\hline & & & & & & 32 & \\
\hline
\end{tabular}

\section{4-15b}


Table 4-6. Cairn density summary.
Hohlraum Studies

\begin{tabular}{|c|c|c|c|c|c|c|}
\hline $\begin{array}{c}\text { Shot } \\
\text { number }\end{array}$ & $\begin{array}{l}\text { Neutron } \\
\text { yield. } \\
\text { millions }\end{array}$ & $\begin{array}{c}\text { Neutron } \\
\text { yield } \\
\text { error }\end{array}$ & $\begin{array}{l}\text { Burn } \\
\text { time } \\
\rho_{\mathrm{p}} \mathrm{AR} \\
\left(\mathrm{g} / \mathrm{cm}^{2}\right)\end{array}$ & $\begin{array}{l}P_{P} \Delta R \\
\text { Error }\end{array}$ & $\begin{array}{c}\text { Bum } \\
\text { time } \\
\text { density } \\
\left(\mathrm{g} / \mathrm{cm}^{3}\right) \\
\end{array}$ & $\begin{array}{c}\text { Density } \\
\text { error }\end{array}$ \\
\hline 38102617 & 0.10 & & & & & \\
\hline 80030602 & 77.00 & & & & & \\
\hline 80030704 & 200.00 & & & & & \\
\hline 80031306 & 100.00 & & & & & \\
\hline 80031403 & 47.00 & & & & & \\
\hline 80031702 & 0.29 & 0.17 & & & & \\
\hline 80031804 & 1.60 & 0.13 & & & & \\
\hline 80032003 & 67.00 & & & & & \\
\hline 80032102 & 74.00 & & & & & \\
\hline 80032605 & 1.90 & 0.21 & 0.0930 & 0.42 & 21.00 & 0.52 \\
\hline 80032608 & 210 & 0.33 & & & & \\
\hline 80032704 & 260.00 & 0.08 & 0.0076 & 0.21 & 2.90 & 0.21 \\
\hline 80032709 & 3.10 & 0.29 & 0.0110 & 0.64 & 4.20 & 0.71 \\
\hline 80040105 & 0.01 & 0.00 & 0.0220 & & & \\
\hline 80040204 & 8.60 & 0.17 & 0.0110 & 0.36 & 4.20 & 0.40 \\
\hline 80040207 & 0.53 & 0.45 & 0.0430 & & & \\
\hline 80040305 & 129.01 & 0.07 & 0.0087 & 0.21 & 330 & 0.21 \\
\hline 80040308 & 2000 & 0.10 & 0.0160 & 0.25 & 630 & 0.27 \\
\hline 80040414 & 0.50 & & & & & \\
\hline 80040902 & 3.40 & 0.26 & 0.0160 & 0.44 & 6.30 & 0.48 \\
\hline 80040906 & 0.32 & 0.41 & 0.1600 & & & \\
\hline 80041005 & 0.74 & 0.27 & 0.0720 & & & \\
\hline 80041010 & 034 & 0.38 & 0.1700 & & & \\
\hline 80072403 & 340.00 & 0.09 & & & & \\
\hline 80072408 & 0.53 & 0.38 & & & & \\
\hline 80072513 & 0.82 & 0.34 & & & & \\
\hline 80073106 & 220.00 & 0.09 & 0.0070 & 0.21 & $2 \pi$ & 0.19 \\
\hline 80080104 & 0.40 & 050 & & & & \\
\hline 80082604 & 0.80 & 0.50 & 0.0810 & 0.72 & $\begin{array}{r}17.00 \\
5.00\end{array}$ & $\begin{array}{l}0.82 \\
0.80\end{array}$ \\
\hline 80082703 & 1.70 & 0.24 & 0.0350 & 0.74 & 500 & 0.80 \\
\hline 80082706 & 0.53 & 0.45 & & & & \\
\hline 88120602 & 4.60 & 0.39 & & & & \\
\hline 89011803 & 0.91 & 0.26 & & & & \\
\hline 89011805 & $\begin{array}{r}10.60 \\
8.40\end{array}$ & $\begin{array}{l}0.11 \\
0.13\end{array}$ & & & & \\
\hline onvizen & & & & & & \\
\hline 89012303 & 0.50 & & & & & \\
\hline 89012509 & 3.60 & $\begin{array}{l}0.14 \\
0.35\end{array}$ & & & & \\
\hline 89013103 & 0.54 & $\begin{array}{l}0.35 \\
0.12\end{array}$ & & & & \\
\hline $\begin{array}{l}89020103 \\
89020203\end{array}$ & $\begin{array}{l}7.60 \\
290\end{array}$ & & & & & \\
\hline 89020504 & 5.01 & 0.16 & & & & \\
\hline 89020703 & 1.30 & 0.23 & & & & \\
\hline 89020804 & 0.21 & 0.71 & & & & \\
\hline 89020905 & 0.77 & 0.19 & & & & \\
\hline 89020908 & 3.40 & 0.32 & 0.0280 & 0.71 & 1200 & 0.42 \\
\hline 89021203 & 6.90 & 0.17 & & & & \\
\hline 89021206 & 0.74 & 0.26 & & & & \\
\hline 89021405 & 1.90 & 0.21 & & & & \\
\hline 89021409 & 1.20 & 033 & & & & \\
\hline 89022004 & 1.30 & 0.31 & 0.0790 & 0.73 & 34.00 & 0.68 \\
\hline 89022006 & 3.40 & 032 & 0.0123 & 0.98 & 4.00 & 1.00 \\
\hline 89022604 & 880.00 & 0.11 & & & & \\
\hline 89022606 & 14.01 & 0.07 & & & & \\
\hline 89022704 & 92.00 & 0.11 & 0.0090 & 0.23 & 3.60 & 0.25 \\
\hline 89022709 & 16.00 & 0.06 & & 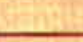 & & \\
\hline 89022802 & 4.00 & 0.18 & & & & \\
\hline 89022808 & 280 & 0.00 & 0.0134 & 0.90 & 4.60 & 0.87 \\
\hline
\end{tabular}


Hohlraum Studies

\begin{tabular}{|c|c|c|c|c|c|c|}
\hline $\begin{array}{c}\text { Shot } \\
\text { number }\end{array}$ & $\begin{array}{l}\text { Neutron } \\
\text { yield, } \\
\text { millions }\end{array}$ & $\begin{array}{c}\text { Neutron } \\
\text { yield } \\
\text { error }\end{array}$ & $\begin{array}{c}\text { Burn } \\
\text { time } \\
p_{\mathrm{p}} \Delta \mathrm{R} \\
\left(\mathrm{g} / \mathrm{cm}^{2}\right)\end{array}$ & $\begin{array}{l}P_{p} \Delta R \\
\text { Error }\end{array}$ & $\begin{array}{l}\text { Bum } \\
\text { time } \\
\text { density } \\
\left(\mathrm{g} / \mathrm{cm}^{3}\right)\end{array}$ & $\begin{array}{c}\text { Density } \\
\text { error }\end{array}$ \\
\hline 89030104 & 0.00 & & & & & \\
\hline 89030108 & 9.40 & 0.15 & & & & \\
\hline 89030202 & 36.00 & 0.17 & & & & \\
\hline 89030213 & 7.10 & 0.18 & & & & \\
\hline 89030505 & 4800 & 0.10 & & & & \\
\hline 89030509 & 210 & 0.19 & & & & \\
\hline 89031207 & 0.51 & 0.37 & & & & \\
\hline 89031211 & 6.01 & 0.00 & 0.0200 & 0.35 & 7.60 & 0.29 \\
\hline 89031304 & 169.01 & 0.09 & 0.0095 & 021 & 390 & 0.26 \\
\hline 89031406 & 3001 & 0.13 & 0.0115 & 0.33 & 4.90 & 0.39 \\
\hline 89031505 & 68.01 & 0.12 & 0.0049 & 0.35 & 1.80 & 0.44 \\
\hline 89031508 & 113.01 & 0.11 & 0.0052 & 0.31 & 1.80 & 0.44 \\
\hline 89031904 & 13200 & 0.10 & & & 1.80 & 1.00 \\
\hline 89031907 & 38.01 & 0.13 & & & & \\
\hline 89032102 & 4.90 & 0.22 & & & & \\
\hline 89032104 & 8.70 & 0.18 & 0.0260 & 0.38 & 10.00 & 0.50 \\
\hline 89032206 & 0.01 & & & & & \\
\hline 89032212 & 149.01 & 0.09 & & & & \\
\hline 89032305 & 148.00 & 0.09 & & & & \\
\hline 89032309 & 0.01 & & & & & \\
\hline 89082306 & 161.01 & 0.10 & & & & \\
\hline \multicolumn{7}{|l|}{89083105} \\
\hline 89090509 & 142.01 & 0.09 & & & & \\
\hline 89090603 & 32.00 & 0.13 & & & & \\
\hline 89090605 & 11.80 & 0.21 & & & & \\
\hline \\
\hline 89090708 & & & & & & \\
\hline 89091004 & 18.80 & 0.15 & 0.0050 & 0.80 & 180 & 0.89 \\
\hline 89091007 & 159.00 & 0.10 & 0.0068 & 0.35 & 260 & 0.46 \\
\hline \multicolumn{7}{|l|}{89091302} \\
\hline 89091304 & & & & & & \\
\hline 89091403 & 29.01 & 0.14 & 0.0130 & 0.46 & 4.40 & 0.57 \\
\hline 89091704 & 720 & 0.19 & & & & \\
\hline 89091706 & 17.01 & 0.12 & 0.0030 & 1.00 & 1.10 & 100 \\
\hline 89091803 & 0.93 & 0.40 & & & & \\
\hline \multicolumn{7}{|l|}{89092104} \\
\hline 89100405 & 32.01 & 0.16 & & & & \\
\hline 89100409 & 49.01 & 0.16 & & & & \\
\hline 89100503 & 2.10 & 0.38 & & & & \\
\hline 89100506 & 6.00 & 0.22 & & & & \\
\hline 89100802 & 050 & & & & & \\
\hline 89100804 & 0.50 & & & & & \\
\hline 89100903 & 220 & 0.36 & & & & \\
\hline 89101105 & 1.50 & 0.80 & & & & \\
\hline 89101504 & 150 & 0.27 & & & & \\
\hline 89101603 & 1.40 & 0.29 & & & & \\
\hline
\end{tabular}

\section{Results from High-Energy Electron-Thermometer (HEET) Measurements}

Introduction. We dedicated 10 successful laser shots to developing the HEET diagnostic. We describe here the preliminary results of this experimental sequence.
The HEET experiments consisted of measuring the characteristic $K_{\mathrm{a}} \mathrm{X}$-ray intensity produced by suprathermal electrons striking a prepared sample (Fig. 4-15) located in either a disk or a Heinz B (half-Cairn 5/8, 20) target geometry. The intensity of the $\mathrm{K}_{\alpha}$ is approximately proportional to the energy in suprathermal electrons striking the material (fluor). Thus, in the first phase of HEET 
diagnostic development, HEET is used as a hot-electron calorimeter. The primary goals of the experiments are

- To determine baseline $\mathrm{K}_{\mathrm{a}}$ signal levels (1) for a simple fluor that is part of a composite disk directly irradiated by the laser and (2) for a composite disk that is the end plate of a half-Caim hohlraum target.

- To determine the contribution to the $K_{\alpha}$ signal by photoionization or anomalous electron transport.

Secondary considerations are

- To verify our earlier Heinz-series experimental results (i.e., to compare the energy in hot-electrons determined by HEET for both a disk and an empty half-Cairn to the energy determined by the

Fig. 4-15. Portion of HEET disk illustrating principle of experiment.

Fig, 4-16. HEET signal disk target. Location of: ALICS $\left(\theta-120^{\circ}\right.$, $\phi$ $\left.-1800^{\circ}\right)$; Henway $(\theta=$ $\left.120^{\circ}, \phi=108^{\circ}\right)$; FFLEX $\left(e-120^{\circ}, \phi-288^{\circ}\right)$; $\operatorname{ZPC} 1 \theta=180^{\circ}, \phi-$ 3609 .

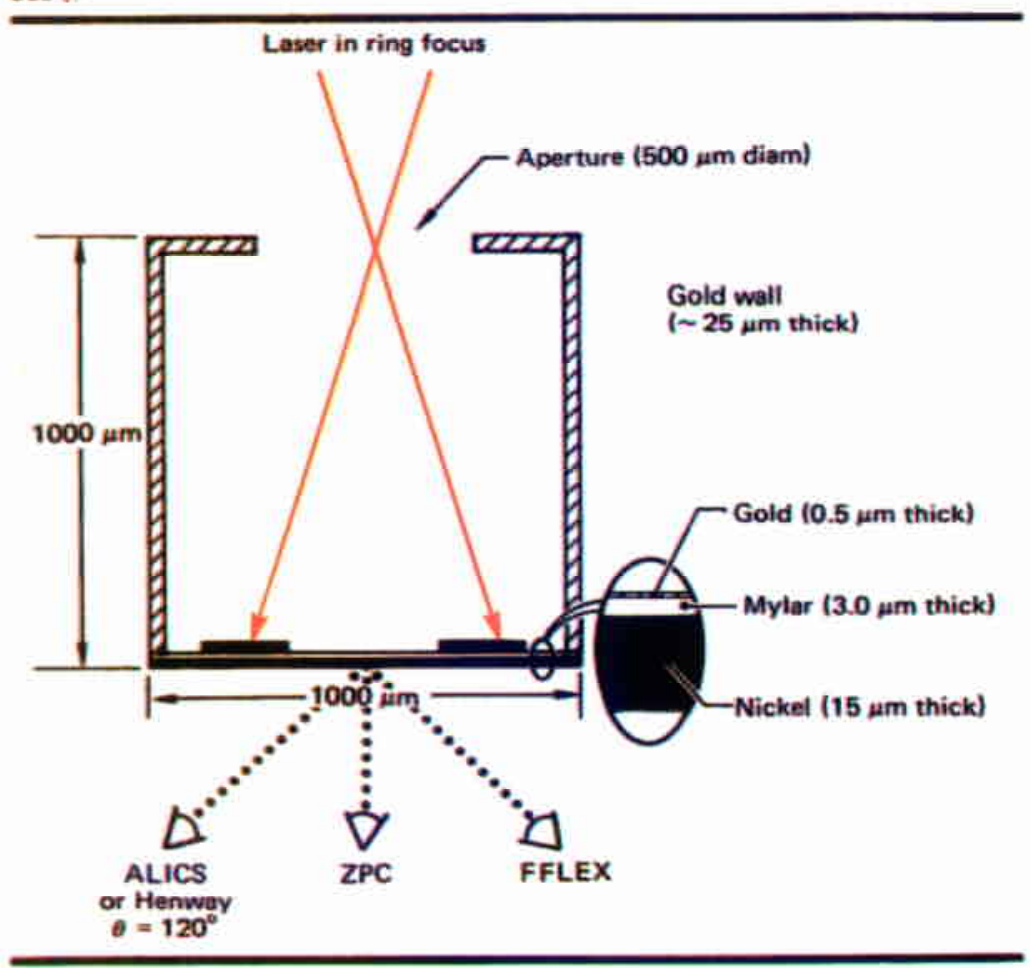

bremsstrahlung method (FFLEX).

- To further our understanding of suprathermal electron transport and production.

Experimental. For all HEET series target shots, we employ the laser parameters that had been used for the Heinz target series, ${ }^{6}$ i.e., 10 Shiva beams in a ring on the base plate of the target (Fig. 4-16). For each beam $\mathrm{E}_{\mathrm{L}}=0.3 \mathrm{~kJ}, \mathrm{I}_{\mathrm{L}}=3 \times 10^{15} \mathrm{~W} / \mathrm{cm}^{2}$, and $\tau_{\mathrm{L}}$ $=600 \mathrm{ps}$. This is the target alignment scheme commonly used for Caim $2.0 \mathrm{H}$ targets.

This series of shots consists of irradiating multilayered signal and null targets. The signal targets are used to determine baseline $K_{\alpha}$ intensity levels. The experimental geometry for the directly irradiated composite disk is shown in Fig. 4-16. The multilayer disk, which also serves as the base plate for the hohlraum target, is carefully chosen to - Provide gold as the laser first-bounce surface and the suprathermal electrongeneration medium. The $0.5-\mu \mathrm{m}$ gold thickness is chosen to avoid laser burn-through until after the peak of the laser pulse. Transport calculations indicate that a 100-keV electron loses about $7 \mathrm{keV}$ in traversing the gold layer. Typical $\theta_{\mathrm{H}}$ values for these targets are $40 \mathrm{keV}$ and about $40 \%$ of the Maxwellian distribution of electrons are stopped by the gold layer. - Discriminate against low-energy electrons ( $\mathrm{E}_{\text {electron }}<10 \mathrm{keV}$ ) by using a 3- $\mu \mathrm{m}$-thick layer of parylene.

- Use nickel as the fluor to efficiently convert electrons having energies $\sim 25 \mathrm{keV}$ into nickel $\mathrm{K}_{\mathrm{\alpha}} \times$ rays $\left(\mathrm{E}_{\mathrm{x} \text { ray }} \simeq 7.5 \mathrm{keV}\right.$ ). The $15 \mu \mathrm{m}$ thickness of nickel is the electron range of $30-\mathrm{keV}$ electrons and at most it attenuates only $1 / \mathrm{e}$ of its own $\mathrm{K}_{a}$ emission (which is viewed in transmission).

We use two Bragg diffraction-type crystal spectrographs to measure absolutely the nickel $\mathrm{K}_{\alpha}$ intensity. The argon-line imaging spectrograph (ALICS) is located close to the target to provide about 30 times greater sensitivity than our general-purpose Henway spectrograph. In these experiments ALICS is always operated without an input slit, thus precluding spatially resolved measurements. The FFLEX instrument measures the bremsstrahlung spectra produced by electrons interacting with both the gold and nickel target constituents. We use the $\theta_{\mathrm{H}}$ 
determinations provided by the FFLEX data in quantitative analyses of HEET data.

In our experiments $\mathrm{K}_{a} \times$ rays can be produced by photoionization of nickel (e.g. produced by bremsstrahlung near $8 \mathrm{keV}$ emanating from gold plasma) and by anomalous electron transport (e.g., electrons circumnavigating the target and striking the unprotected rear surface of the nickel sample).

Targets were designed to test these effects and the principal designs are illustrated in Fig. 4-17. First, a thick Mylar layer is inserted in front of the nickel fluor to stop electrons having kinetic energies $\sim 100$ $\mathrm{keV}$ that would come directly through the target [Fig. 4-17(c)]. Two different thicknesses are used (127 and $254 \mu \mathrm{m}$ ) because of a theoretical uncertainty in the electron-stopping power of Mylar. Any resultant $\mathrm{K}_{a}$ signal would be produced by either photoionization from bremsstrahlung produced in the gold layer (since the Mylar layer is "thin" to photons near the nickel $\mathrm{K}$-shell binding energy, $\mathrm{E}_{\mathrm{K}}=8.33 \mathrm{keV}$ ) and/or by electrons reaching the rear of the target. Protected null targets [Fig. 4-17(d)] are also used in the HEET series to check for this anomalous electron transport. This design protects the rear of the target from electrons that might impinge on the rear side.
Because $K_{a} \times$ rays can still be transported out of the Mylar layer on the rear side of the target, a photoionization measurement is still made. If anomalous electron transport proves significant and photoionization does not, we will have to protect our signal targets by including $127-\mu \mathrm{m}$-thick Mylar layers on the rear side [Fig. 4-17(b)]. If photoionization does not contribute significantly to our $\mathrm{K}_{\alpha}$ signal, then we can determine the amount of electron transport straight through the target compared to that going around the target. More precisely, a comparison of the $K_{a}$ yield from a null disk having an unprotected rear surface [Fig. 4-17(c)] with the yield from a signal disk having a protected rear surface [Fig. 4-17(b)] can provide a quantitative measure of the electron transport around the multilayer target. A similar determination can then be made in the Heinz B geometry.

\section{Results and Discussion}

Qualitative Results. Figure 4-18 shows typical spectral results from the HEET senies. A superimposed nickel $x$-ray spectrum illustrates the differences between (a) a spectrum obtained by the Henway spectrograph during the HEET series (viewed in transmission) and (b) a spectrum obtained by direct laser irradiation of a nickel disk (viewed from the front). The spectra exhibit two important features. (1) All thermal (a) Signal target

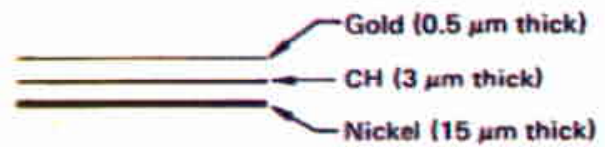

(b) Protected signal target

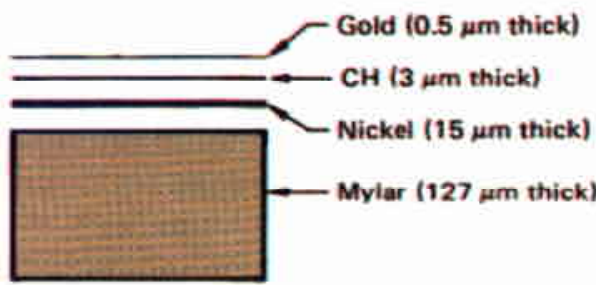

(d) Protected null target

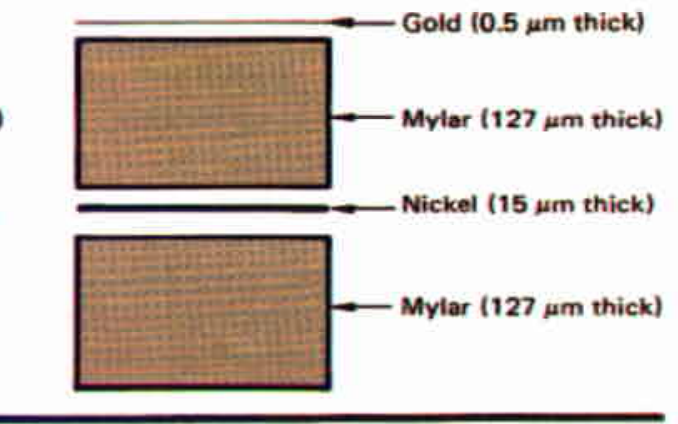

Fig. 4-17. $\mathrm{K}_{a}$ fluor components for various geometry targets. (c) Null target

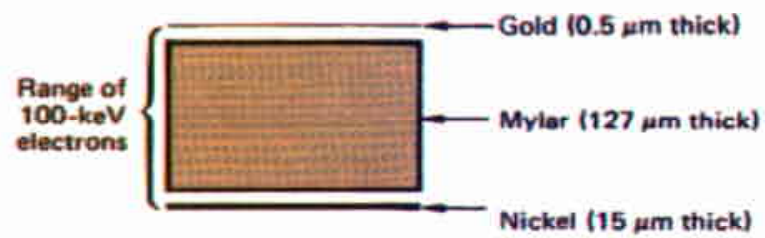


Fig. 4-18. Sample of (a) $\mathrm{K}$ data for nickel from HEET experiments and (b) backlighting series.

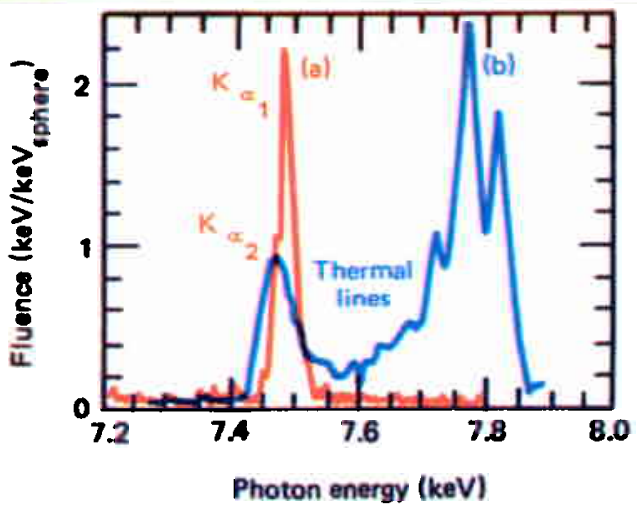

$x$-ray lines are absent in the spectrum from the HEET target (a Heinz B signal target in this case; see Fig. 4-17). This implies that no thermal plasma is present in the nickel layer during the time of $K_{\alpha} x$-ray emission. (2) The signal-to-background level associated with the HEET target measurement is very good. There is no observable $x$-ray continuum and the observed background observed is film noise. Having this high signal-to-noise ratio, we can easily observe a signal 50 times weaker than that shown in Fig 4-18. The ALICS instrument, which has significantly higher efficiency, indicates a very high signal level on this shot. However, the signal-to-noise ratio is severely reduced, presumably because of extensive film fogging by high-energy $x$ rays. Consequently we are experimenting with special films to eliminate this problem.

A signal disk, a thin null disk (127- $\mu$ m-thick $\mathrm{CH})$, and a thick null disk (254- $\mu$ m-thick $\mathrm{CH}$ ) all give nearly the same nickel $\mathrm{K}_{\alpha} \mathrm{x}$-ray yield. According to the design of our experiment, this implies that photoionization and/or anomalous electron transport are major contributors to our signal. Therefore a 127- $\mu \mathrm{m}$-thick layer of Mylar is placed in the back of the target shown in Fig. 4-17, which thus forms a protected thin null target. As a result we observe no $K_{\alpha}$ signal with the HEET-protected null disk [Fig. 4-17 (d)]. This indicates that photoionization is insignificant and anomalous electron transport is appreciable.

To obtain no $K_{\alpha} x$-ray yield (i.e., a null) with the Heinz B target, we also require that the back of the target be protected with $127 \mu \mathrm{m}$ of Mylar. Therefore we conclude that hohlraum target geometries are also affected by anomalous electron transport. - For hohlraum targets, however, we observed a $\mathrm{K}_{\alpha}$ signal level with a null target that was about $20 \%$ as intense as that observed with a signal target. This residual signal level can be the result of either increased photoionization that is attributable to the target geometry or to the presence of very-high-energy electrons.

Having shown that photoionization is not significant and that a null can be obtained, we can determine the number of electrons transporting through our targets relative to the amount reaching the rear of the target. Because a quantitative measurement requires a model for the electron transport to interpret the $\mathbf{K}_{\alpha}$ results, we discuss here only model-independent (i.e., qualitative) discoveries. A L.ASNEX prediction for energy transport and the corresponding quantitative results are discussed below.

Comparing the $\mathbf{K}_{\alpha}$ yield from an unprotected null disk (whose signal level results from electrons reaching the rear of the target) to the $\mathbf{K}_{\alpha}$ yield from a protected signal disk (whose signal results from electrons traversing the gold and 3- $\mu \mathrm{m}$-thick parylene layers) indicates that the signal levels are equal. Furthermore, for the hohlraum target geometries (namely, the Heinz B), we have also measured significant $K_{\alpha} x$-ray yield stemming from electrons reaching the rear side of the target. These are important observations since, unlike theoretical prediction, they illustrate that elextron transport can be appreciable to portions of the target other than the laser-plasma interaction area.

Quantitative Results. Quantitative determination of such quantities as $E_{\text {hot }}$ or $f_{\text {hot }}$ from the $K_{a}$ technique require knowing suprathermal electron transport from the laser-plasma interaction region to the nickel HEET sample. In the following discussion we assume that the electrons are transported isotropically after formation and that they comprise a Maxwellian velocity distribution. Making these assumptions, we use LASNEX to calculate the energy deposition by the hot electrons in the various target components. Using $O_{H} \simeq 40 \mathrm{keV}$ (which is measured by FFLEX in these experiments), LASNEX predicts that about $60 \%$ of the enengy in hot electrons (present at the laser-plasma interaction region) is actually deposited in the nickel sample. Most of the remaining $40 \%$ of the energy is deposited in the gold layer. LASNEX does not predict any transport to the rear side of the target. Furthermone, the average depth of 
formation of $K_{\alpha} \times$ rays in the nickel sample (measured from the laser interaction side) is calculated to be $(x) \simeq 5 \mu \mathrm{m}$. The energy deposition in various layers and the average $K_{\alpha}$ formation depth $(x)$ are important quantities to aid in the interpretation of our results and are incorporated in all the discussion below.

Some $x$-ray transport corrections are also required to analyze the HEET $\mathrm{K}_{\alpha}$ data. The observation angle used by both the Henway and ALICS spectrographs requires correction for the effect of absorption of nickel $\mathrm{K}_{\alpha}$ in both the nickel and Mylar layers. At an observation angle of $60^{\circ}$ relative to an axis normal to the surface of the disk, nickel $K_{\alpha} \times$ rays must pass, at most, through $30 \mu \mathrm{m}$ of nickel and $254 \mu \mathrm{m}$ of Mylar (when a Mylar protective layer is on the rear of the target). The resultant absorption in Mylar is only $20 \%$. Nevertheless, the corrections are made when necessary.

The compensation for the transport through the nickel, however, is not as simple. Formally, we must determine the average depth $(x)$ where $x$-ray formation takes place and correct for the attenuation through the remaining thickness of nickel. As mentioned previously, the average depth was calculated by LASNEX to be $5 \mu \mathrm{m}$. Because of our observation angle, the transport path length for $x$ rays produced by electrons striking the front side is $20 \mu \mathrm{m}$, which leads to a $57 \%$ transmission factor. Similarly, for $x$ rays produced by electrons striking the rear side, the transmission factor increases to $76 \%$. Obviously our measurements are more sensitive to electrons striking the rear side of the target. We used these transport corrections in analyzing all our data. The maximum error in the transmission correction produced by uncertainties in $\langle x\rangle$ is barely significant since full transport (through $30 \mu \mathrm{m}$ of nickel) would imply $80 \%$ absorption as opposed to $44 \%$ if the $x$ rays pass through the center of the slab (i.e., through $15 \mu \mathrm{m}$ of nickel).

Figure 4-19 shows the conversion efficiencies for calculating the energy in suprathermal electrons from the nickel $K_{\alpha}$ yields. For monoenergetic electrons, these conversion efficiencies agree with experimental and theoretical values published by Green and Cosslett. ${ }^{7}$ In practice we must have a separate measurement of the suprathermal electron temperature $\theta_{\mathrm{H}}$ to determine $\eta_{\text {electron } \rightarrow x \text { ray }}$ from the curves in Fig. 4-19. We use FFLEX results to determine $\theta_{\mathrm{H}}$ for all the present measurements.

Quantitative summaries of all the HEET data are shown in Tables 4-7 and 4-8. The absolute energy in hot electrons, as determined by HEET, is somewhat uncertain by a factor of 2 for the absolute $K_{\alpha}$ intensity and probably another factor of 2 in the conversion efficiency. We have designed subsequent experiments using an electron source of known energy that should at least eliminate the uncertainty in the conversion efficiency. The $\theta_{H}$ values have been determined from preliminary filter fluorescer $x$-ray spectra and should be accurate to $\pm 10 \mathrm{keV}$. In all our analyses we use a constant conversion efficiency of

$$
\begin{aligned}
\eta_{\text {electron } \rightarrow x \text { ray }} & =3.8 \times 10^{-3} \mathrm{~J} \text { of } \\
& \mathrm{K}_{\alpha \times \text { ray } / \mathrm{J} \text { of electron-sphere },}
\end{aligned}
$$

which incorporates a $50 \%$ error resulting from the variation of $\theta_{\mathrm{H}}$ among targets. Therefore,

$$
\mathrm{E}_{\text {hot }}^{\text {HEET }}=\mathrm{E}_{\mathrm{K}_{\alpha}} / \eta_{\text {electron } \rightarrow \mathrm{x} \text { ray }},
$$

where we have ignored the small variation in $\eta_{\text {electron } \rightarrow x \text { ray }}$ as a function of $\theta_{\mathrm{H}}\left(\theta_{\mathrm{H}} \sim 40\right.$ to $50 \mathrm{keV}$; see Fig. 4-19). The fraction of incident laser energy converted into hot electrons is denoted by $\mathrm{f}_{\text {hot }}$

In Tables 4-7 and 4-8 we also list the energy in hot electrons as determined from the so-called Kruer bremsstrahlung formula:

$E_{\text {hot }}^{\text {HEET }}=\frac{\mathrm{I}_{x}^{\text {FFLEX }}\left(\theta_{h}\right)}{\left(5 \times 10^{11}\right)(Z / 79)}$

where $I_{x}^{\text {FFLEX }}\left(\theta_{h}\right)$ is the bremsstrahlung $x$-ray

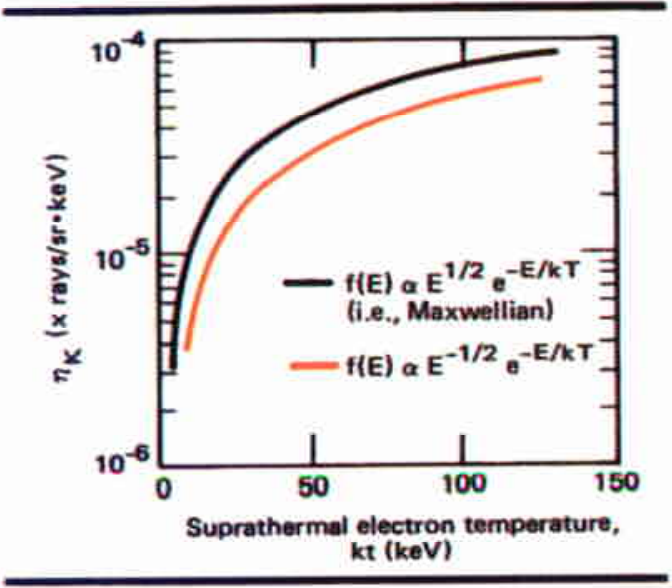

Fig. 4-19. Conversion efficiency for changing measured nickel $\mathrm{K}_{\alpha}$ yield into energy deposited by suprathermal electrons as function of electron energy or temperature kT. 
Table 4-7. HEET quantibative results

\section{Hohiraum Studies}

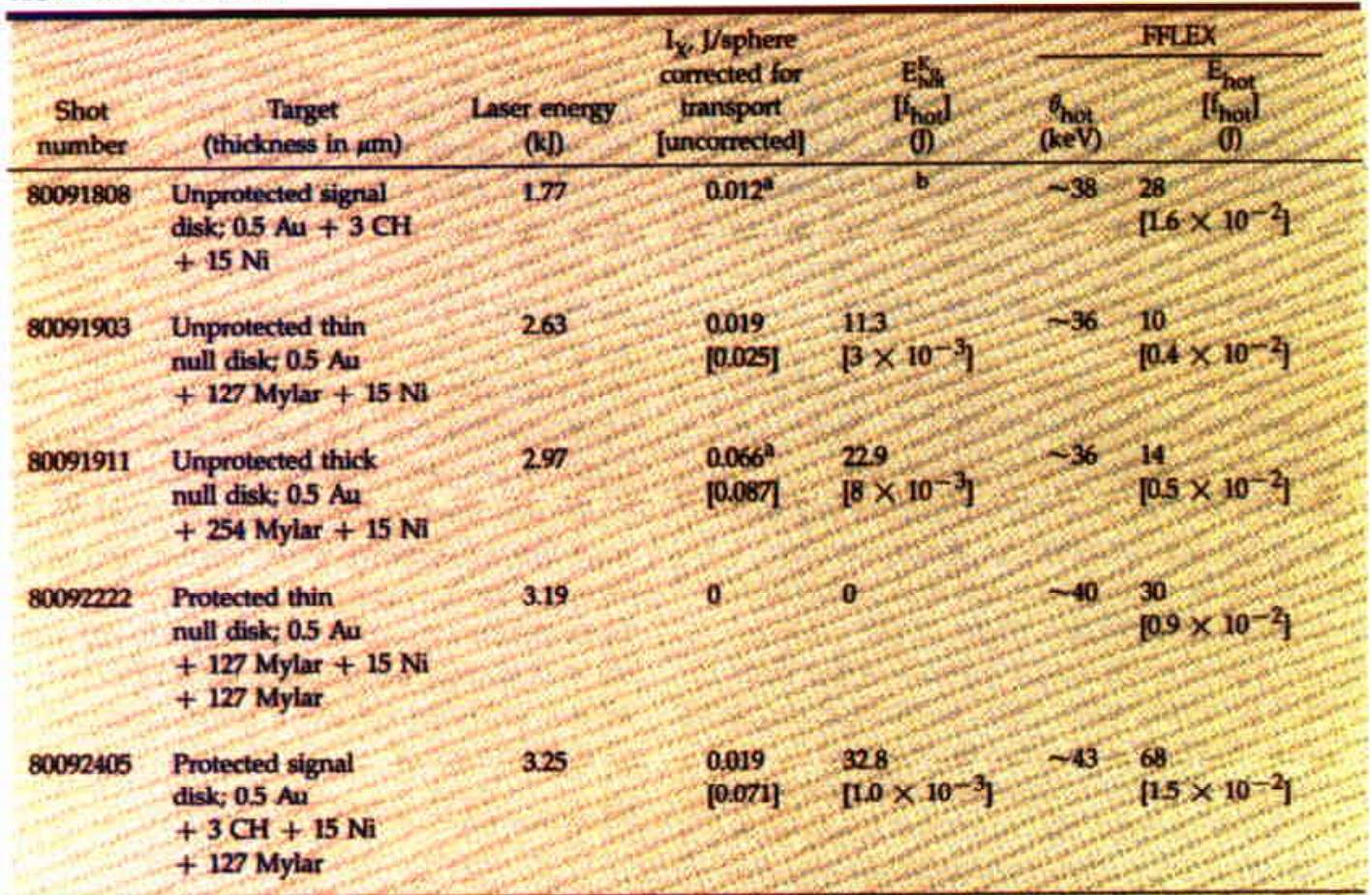

"These points were measured by ALICS only and the ALLCS calibration was suspect.

bunable to determine.
Table 4-8. HEET quantitative data summary (hohlraum targets).

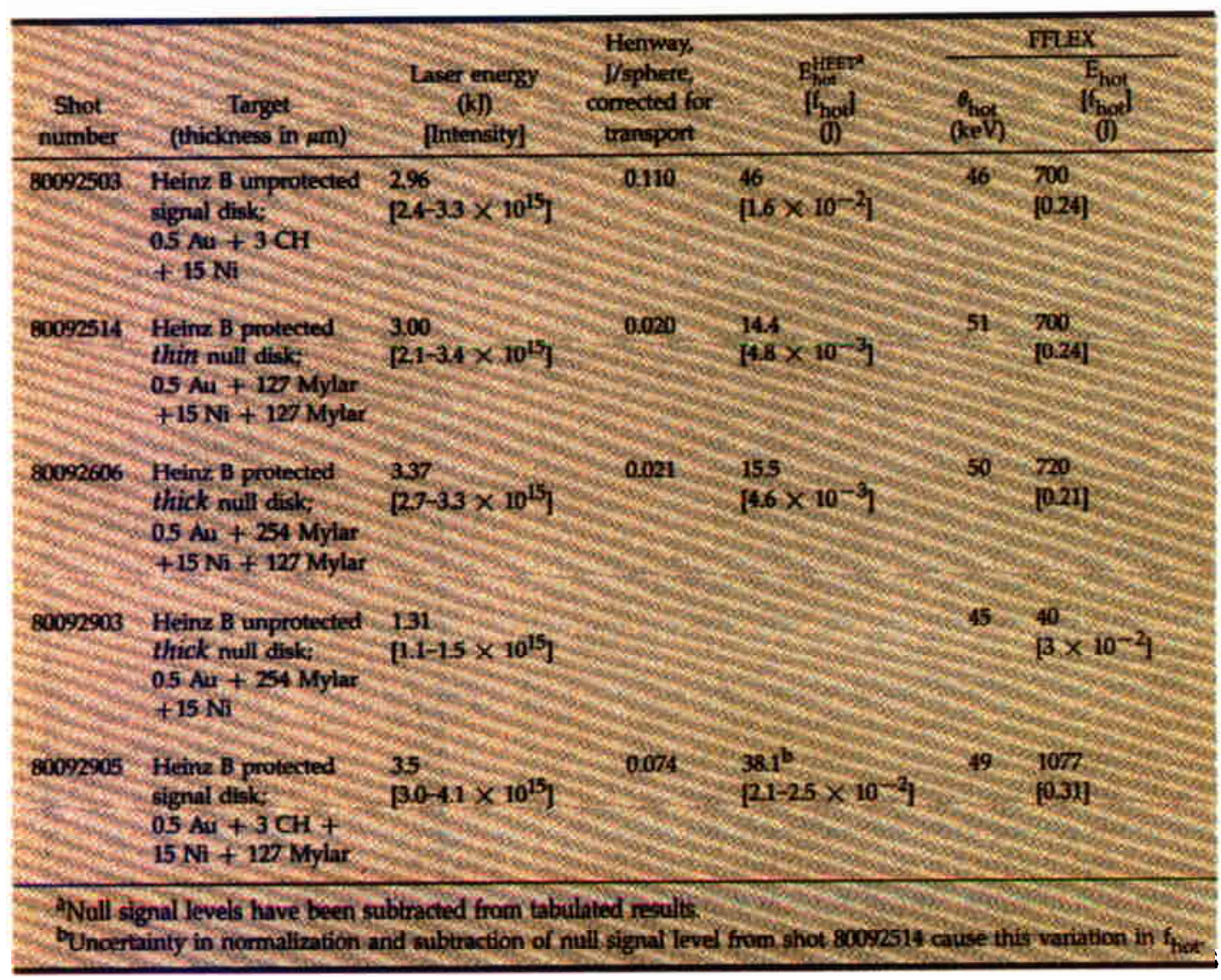


intensity $\mathrm{keV} /(\mathrm{keV}$ sphere) at a photon energy equal to $\theta_{\mathrm{h}}$ and $\mathrm{Z}$ is the atomic number of the material producing the bremsstrahlung. For our multilayer targets it is difficult to apply this formula accurately because of the presence of gold $(Z=79)$ and nickel $(Z=28)$ layers. (Note that bremsstrahlung production in parylene is negligible.) Using the assumptions discussed earlier, LASNEX predicts that for a disk, $57 \%$ of the $40-\mathrm{keV} \times$ rays come from the nickel layer and the remainder come from the thin gold layer. Obviously, if electrons have curved trajectories so that they preferentially deposit energy in the gold, then all the bremsstrahlung would be produced in the gold layer. Under these unlikely conditions, the tabulated values of $\mathrm{E}_{\text {hot }}^{\text {FFEX }}$ would be halved. Unfortunately, for the Heinz B or hohlraum target geometries the situation is completely different since thick gold walls $(\sim 25 \mu \mathrm{m}$ thick) are present almost everywhere except at the entrance aperture and at the laser interaction surface. For this geometry we assume all the bremsstrahlung is produced in the gold.

The bremsstrahlung measurement is also compromised by electrons orbiting to the rear side of the target. LASNEX calculations do not predict this effect. Our HEET measurements will show that the number of electrons orbiting is at most $20 \%$ of those transported through the target. As a consequence we have not corrected the FFLEX data to account for additional bremsstrahlung production in the nickel arising from electrons reaching an unprotected rear side of a target.

Some quantitative results from the HEET disk targets deserve emphasis. As shown by shot 80092405 (Table 4-7), the energy in suprathermal electrons transported directly to the nickel is small: $f_{\text {hot }}=4 \times 10^{-3}$. In comparison with shot 80091903 (and assuming linear scaling of $E_{\text {hot }}$ with $E_{\text {lased }}$ ), we can determine that roughly $1 / 6$ of this amount of energy is transported to the rear side of the target. Thus the total energy in suprathermal electrons incident on our nickel wafer from any direction is still small. The total spatially integrated $f_{\text {hot }} \simeq 0.01$. (For a disk target $\mathrm{f}_{\text {hot }}$ may not be the total fraction of laser energy converted into hot electrons. We only measure the electrons that interact with the target, ignoring any losses due to corona plasmas or fast ions.)
For disk targets, we measure only slightly less energy in suprathermal electrons using the $K_{\alpha}$ technique than we obtained using FFLEX. We also found similar agreement for measurements made using pure titanium, nickel, and zinc disks. This outstanding agreement supports our assumption about the energy deposition in various target components and also establishes the credibility of the HEET measurement.

For the hohlraum targets, we again observe significant electron transport to the rear of the target. Comparison of shots 80092514 and 80092905 reveals that for every 100 electrons striking the front of the HEET sample, 14 strike the rear side if we assume linear scaling of $\mathrm{E}_{\text {hot }}$ with $\mathrm{E}_{\text {laser }}$ or that 60 electrons strike the rear side if $E_{\text {hot }}$ scales as $\mathrm{E}_{\text {laser. }}^{3}$.

This observation implies that from 0.4 to $1 \%$ of the laser enengy is converted into hot electrons that reach the rear side of the target.

Comparing the energy in hot electrons measured for a disk with that measured for a Heinz B target illustrates an interesting result: $\mathrm{E}_{\text {hot }}^{\text {HEET }}$ (Heinz B) is only 2.5 times greater than $\mathrm{E}_{\text {het }}^{\mathrm{HET}}$ (disk). This factor of $\mathbf{2 . 5}$ could result from the greater absorption of laser light that is noted for half-Caim geometry vs that absorbed for disk geometry. Curiously, when the same comparison is made using results from the FFLEX data, the bremsstrahlung measurement indicates $\mathrm{E}_{\mathrm{hot}}^{\text {FrLX }}$ is 20 times greater for the Heinz B target than for a disk. (This is apparent on comparing energy-normalized values from shots 80092905 and 80092405 .)

Assuming that both the bremsstrahlung and the $\mathrm{K}_{\alpha}$ measurements are accurate, this discrepancy may illustrate some interesting properties of suprathermal electron transport and production. First, there is no reason to have assumed a priori that for a hohlraum target, $\mathrm{E}_{\mathrm{hot}}^{\mathrm{HHFT}}$ would have equaled $\mathrm{E}_{\text {hot }}^{\mathrm{FLLX}}$. The $\mathbf{K}_{\alpha}$ measurement is only sensitive to those electrons that are transported directly forward from the laser-plasma interaction region into the nickel sample, L.e., it is a spatially localized measurement. On the contrary, the FFLEX measurement observes bremsstrahlung $x$ rays from wherever suprathermal electrons collide with the predominantly gold target. If we assume that suprathermal electrons are generated at the laser first-bounce surface (which is at the 
critical-density surface of the $0.5-\mu \mathrm{m}$ gold layer) and that they are isotropically emitted, then $\mathrm{E}_{\text {hot }}^{\text {FFEX }}$ should be twice as large as $\mathrm{E}_{\mathrm{hot}}^{\mathrm{HET}}$. We arrive at this number by assuming that half the electrons go in the direction of the nickel sample (which under these conditions subtends $\Delta \Omega / \Omega=2 \rho s r$ ) while an equal number of electrons produce bremsstrahlung at the cylinder walls and aperture. Instead, the measured ratio of $\mathrm{E}_{\text {hot }}^{\text {FFI }}$ to $\mathrm{E}_{\text {hot }}^{\mathrm{HEET}}$ is 13 to 15 (depending on uncertainties in subtracting the null signal from the $K_{u}$ yield). Thus, the bremsstrahlung measurement predicts 13 to 15 times more energy in suprathermal electrons. Clearly, to be consistent with these measurements, the electrons cannot be produced at the target base plate and emitted isotropically.

If we assume that the electrons are still emitted isotropically but are produced near the center of the enclosed target volume, then this is a case that supports electron production by the stimulated Raman scattering of laser light in low-density plasma (1/4 $\mathrm{N}_{\mathrm{c}}$ or less). Under these conditions the available surface area of gold exceeds that of the nickel by a factor of about 6. Multiplying this result by the observed discrepancy between $K_{a}$ and bremsstrahlung techniques for the HEET disks (FFLEX results are about $50 \%$ higher) implies that $\mathrm{E}_{\text {hot }}^{\text {FFEX }} \simeq 9 \mathrm{E}_{\text {hot }}^{\mathrm{HEET}}$ which is still somewhat smaller than the observed value of 13 to 15 . The remainder could be partially accounted for by considering the large external surface area of the gold radiation case that is unprotected from orbiting electrons. Another mechanism that could account for the discrepancy is the preferential (nonisotropic) transport of electrons down the density gradient established at the target back plate and into the gold cylinder walls. Other mechanisms such as preferential orbiting of electrons in the thin gold layer could also be invoked to explain the large $\mathrm{E}_{\text {hot }}^{\text {FrEX }}$. (This argument only applies to hohlraum targets; the two experimental methods agree much better for disks.) Only by performing further measurements can we fully understand the electron emission isotropy and production mechanism. For example, constructing a Heinz B target out of different $\mathbf{Z}$ components and using the $K_{\alpha}$ technique could obviously tell us how much electron energy goes into certain directions.
Summary.

- HEET is a viable technique for studying suprathermal electron collisions with ICF target components.

- The HEET sensitivity limit is about $0.5 \mathrm{~J}$ for electrons having kinetic energies of $25 \mathrm{keV}$ or higher (up to about $100 \mathrm{keV}$ ).

- Electron transport to the rear side of both disk and hohlraum targets is substantial. Measurements using the $K_{\alpha}$ technique allow us to

- Study the electron preheat levels at the D-T-filled fuel-capsule location in a high-density target.

- Determine suprathermal electron transport by building a Heinz B "can" using different $K_{\alpha}$ fluor components.

- Measure the spatially localized electron energy distribution $F(E)$ using a specially prepared HEET sample.

- Temporally resolve the $K_{\alpha}$ line to determine time history of suprathermal electron transport at different target locations.

\section{Authors: D. L. Matthews and R. L. Kauffman}

Nova Cone-Angle Experiments

The Nova cone-angle experiments explore the possibility that the large cone angle of the Nova beams may cause refractive losses in the plasma plumes that in turn lead to diminished absorption in hohlraums.

Figure 4-20 shows two of the beams in the proposed Nova beam configuration irradiating a cylindrical hohlraum. We are concerned that the marginal rays of each beam may enter at at so shallow an angle to the hohlraum surface that they will be refracted away from the entrance hole by the plasma plume. The amount of refraction would depend on the plume density and density gradients. Our cone-angle experiments tried to simulate in part a Nova beam/hohlraum environment and to ascertain if refraction takes place. We intended to measure any increase in scattered light and any degradation of hohlraum absorption of laser light.

The experiments performed on Shiva used a beam configuration different than that shown in Fig. 4-20. Hence, special targets were designed and fabricated to simulate the shallow angle between a marginal 
ray and the hohlraum surface. Figure 4-21 shows the targets, designated Cone $\mathrm{A}$ and Cone $\mathrm{B}$, and the alignment configuration of the Shiva beams.

The Cone A target has a diameter of $2500 \mu \mathrm{m}$ and mid-height of $2500 \mu \mathrm{m}$. Because the end cap is tilted, some of the 10 beams of a Shiva beam cluster have marginal rays within 35 to $50^{\circ}$ of the surface plane (as is the case in Fig. 4-20). LASNEX hydrodynamics computer code simulations indicate that this Shiva hohlraum, when irradiated by a 4-kJ/5-ns pulse, will fill to plasma densities higher than calculated for Nova hohlraums. At the peak of a 5-ns pulse, LASNEX calculates a hohlraum average density of $n / n_{c} \simeq 0.06$. When

two-thirds of the laser-pulse energy is delivered, the average density rises to $n / n_{c} \simeq$ 0.125 . This compares to a LASNEX calculation for the implosion of a 160-eV Apollo ignition capsule, which reaches $n / n_{c}<0.04$ with two-thirds of the energy delivered into the hohlraum. Even at this higher density, we expect no significant refraction problem. Actual densities at the laser entrance hole and beyond should be a factor of severalfold lower than the hohlraum average density because the plasma expands and accelerates as it passes through this orifice. The expected absorption for this size hohlraum with a perpendicular end cap is about $80 \%$. A significantly lower value indicates that refraction of the beams is taking place. As shown in Fig. 4-21, the target also has a diagnostic hole for measuring radiation temperature, which is also used as an indicator of absorption.

Four experiments were completed with the Cone A targets. Two of the targets used an irradiation scheme slightly different from that shown in Fig. 4-21. One of the inner beams was positioned so that it irradiated the entire end cap. The resulting additional blowoff was expected to enhance any exist-
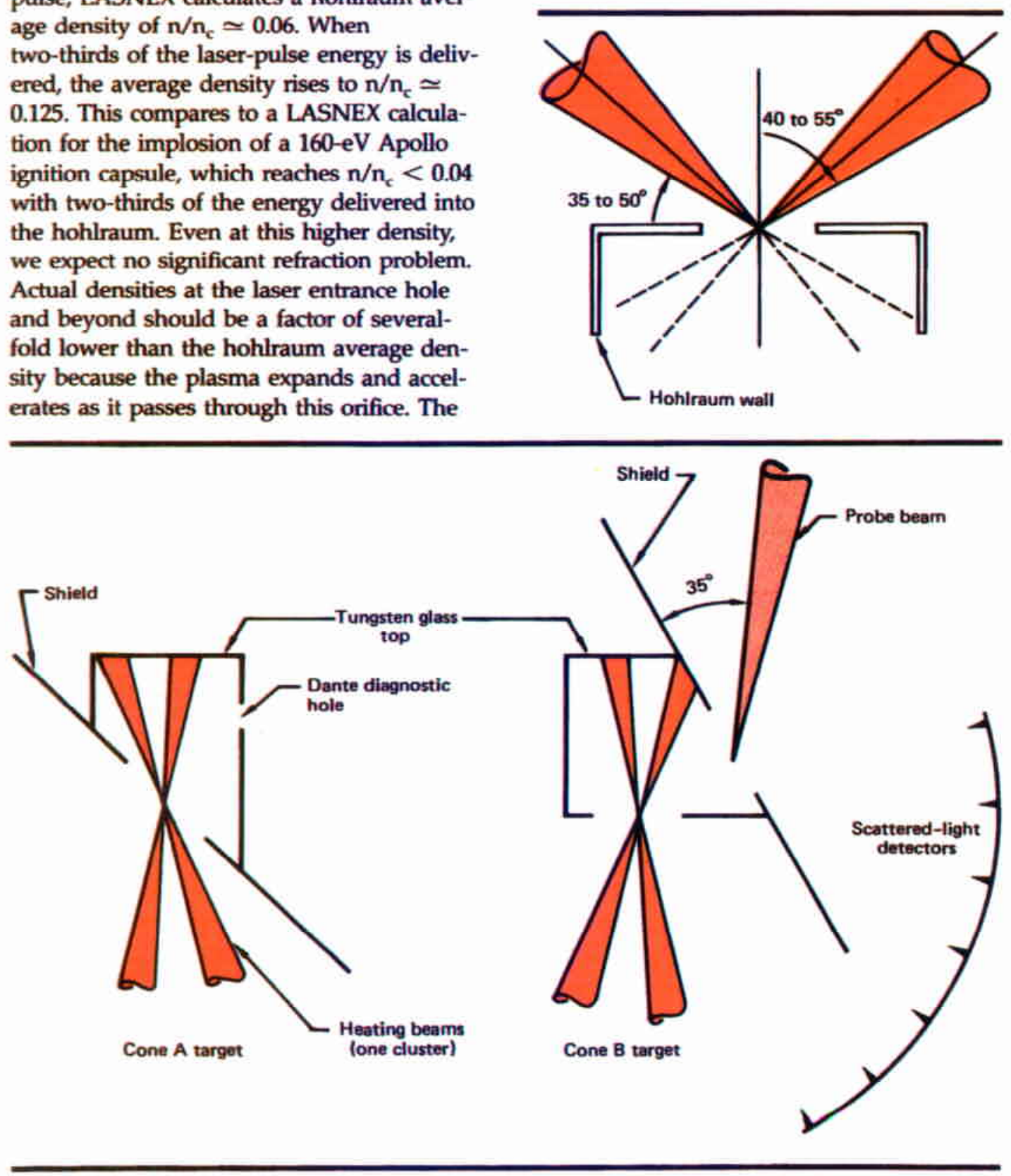

Fig. 4-20. Nova beams irradiating hohlraum.

Fig. 4-21. Targets for Nova cone-angle experiments. 


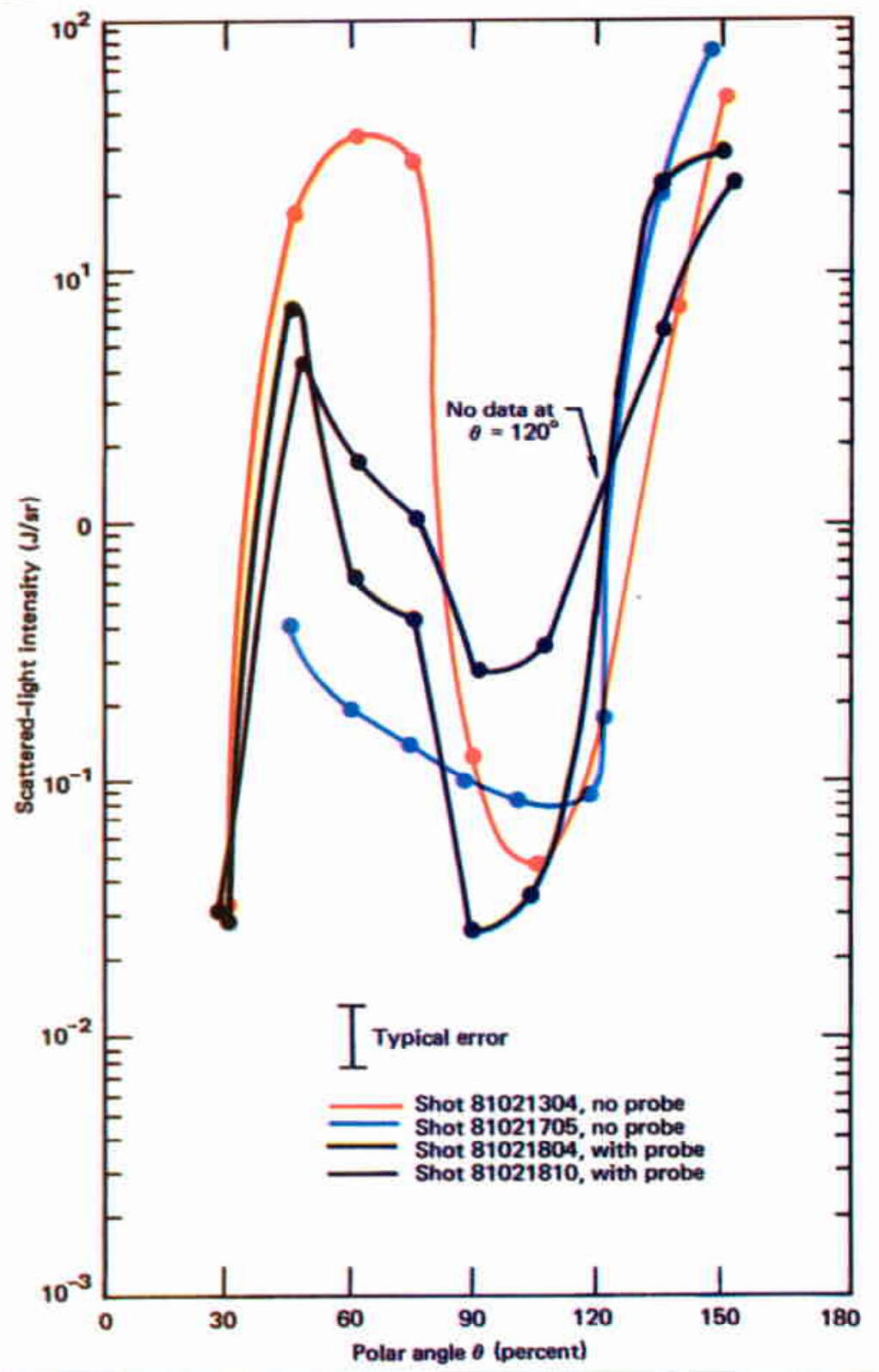

ing refraction effects. Table 4-9 presents the experimental results and the laser parameters. The absorption figures show no evidence of refraction effects as manifested by a degradation of absorption.

The Cone B target was used to measure refraction directly rather than by measuring degradation of absorption. As shown in Fig. 4-21, one cluster of beams is used to heat the hohlraum with a nominal 4-kJ/5-ns pulse and one beam of the opposite cluster is used as a probe. An array of photodiodes in the plane defined by the probe beam axis and the normal to the slanted surface is used to monitor for refracted light. The probe is aimed at the center of a $1000 \mu \mathrm{m}$ diagnostic hole and can be refracted by the plume emanating from the hole.

Four experiments were completed with the Cone B target (Table 4-9). Two were reference experiments in that no probe was used. The experiments were used to acquire baseline values for the scattered-light detectors. Two experiments were then conducted with the probe. No difference was noted between the scattered-light distributions for the "probe" target and the "no-probe" targets in the hemisphere opposite the incoming probe beam. This is illustrated in Fig. 4-22, which shows the scattered-light distribution for all four targets. If the approximately $520-\mathrm{J}$ probe pulse had been scattered uniformly into the forward $2 \pi \mathrm{sr}$, we would expect detector readings of about $150 \mathrm{~J} / \mathrm{sr}$ from sensors located from $\theta \simeq 60^{\circ}$ to $\theta$ $\simeq 150^{\circ}$. From these data we concluded that no significant refraction occurred for these targets.

Fig, 4-22. Scattered light distribution-cone B targets for diode array at $\theta$ $=126^{\circ}$

Table 4-9. Nova coneangle experiments.

\begin{tabular}{|c|c|c|c|c|c|c|}
\hline \multirow{2}{*}{$\begin{array}{c}\text { Shot } \\
\text { number }\end{array}$} & \multicolumn{3}{|c|}{ Tanget } & \multicolumn{2}{|c|}{ Lase } & $\begin{array}{l}\text { Absorption by } \\
\text { photodiode and } \\
\text { reflected-beam }\end{array}$ \\
\hline & Type & Cone & Scale & Beams & (kJ/ns) & (\%) \\
\hline 81020605 & Heinz 44 & A-1 & 25 & $1-10$ & $3.64 / 5$ & 86 \\
\hline 81020904 & Heinz 45 & A-1 & 25 & $1-10$ & $4.01 / 5$ & 88 \\
\hline 81021203 & Heinz 46 & $A-1$ & 25 & $1-10$ & $3.71 / 5$ & 72 \\
\hline 81021205 & Heinz 47 & $A-1$ & 25 & $1-10$ & $3.46 / 5^{a}$ & 83 \\
\hline 81021304 & Heinz 50 & B-1 & 25 & $1-10$ & $364 / 5^{b}$ & 87 \\
\hline 81021705 & Heinz 54 & B-1 & 25 & $1-10$ & $356 / 5^{b}$ & 82 \\
\hline 81021804 & Heinz 55 & B-1 & 25 & $1-10$ & $3.40 / 5^{e}$ & 83 \\
\hline 81021810 & Heinz 51 & B-1 & 25 & $1-10$ & $3.63 / 5^{d}$ & 84 \\
\hline 81021903 & Heinz 56 & A-1 & 25 & $1-10$ & $3.71 / 5^{\mathrm{a}}$ & 90 \\
\hline
\end{tabular}

$49+1$ align.

bo probe.

brobe: beam 13, $520 \mathrm{~J}$.

dProbe beam 13,516 J. 
A second set of experiments will be performed next year using smaller targets. These targets are equivalent in volume to a 2-mm high by 2-mm diam cylinder. LASNEX calculations indicate that, when irradiated by a $4-\mathrm{kJ} / 5$-ns pulse, these hohlraums will fill to about $0.25 n_{c}$ before the peak of the pulse. Plumes fed by plasma of this density should produce significant refraction effects. Since this fill density is many times that expected for Nova, the experiments should be a severe test of the utility of the currently proposed Nova beam configuration.

Author: J. M. Auerbach

Major Contributors: K. R. Manes and D. W. Phillion

\section{Summary of Hohlraum Experiments}

During the past year we extended our hohlraum-scaling data base to include $1.06-\mu \mathrm{m}$ experiments having laser pulse lengths up to 6 ns and to include experiments with $2 \omega(0.53 \mu \mathrm{m})$ and $3 \omega(0.35 \mu \mathrm{m})$ at $0.6-n s$ pulse lengths. The scaling of the hard $\mathrm{x}$-ray levels from these targets is consistent with a hohlraum-filling model of hotelectron production except at very short pulse length $(r \simeq 100 \mathrm{ps})$. We experimentally verified both the predicted large reduction in hot-electron fluxes at short wavelengths and the pulse-length dependence of the hot-electron production predicted by the filling model. We also found that the temperature of the hot electrons scales as $\left(\mathrm{D}^{2}\right)^{1 / 3}$, as predicted by simulations of effects near $(1 / 4) \rho_{c}$. The Raman-scattered light scales roughly with the hot-electron fraction, showing the importance of this plasma process in hohiraums. We see Raman-shifted spectra having as much as 15 to $20 \%$ of the incident laser light.

The HEET $K_{\alpha}$ experiment has given us an alternative to high-energy bremsstrahlung as a technique for measuring hot-electron fluxes. This technique potentially can be used to obtain time, space, and spectral information about high-energy electrons. The applicability of the technique to hohlraums will depend on the ratio of photoionization to electron-impact ionization of the fluor.
This ratio depends on laser wavelength, hohlraum temperature, and hot-electron levels in specific designs.

The Nova cone-angle experiment provided preliminary information that the Nova beam geometry will result in high absorption in hohlraums. Using a specially designed hohlraum that, when irradiated by Shiva, we calculate to have a density twice that of a typical Nova hohlraum, we see neither reduction absorption nor an observable refraction of a probe beam incident at the Nova beam angle. However, because of interference with scattered light from the heating beams, we would not see low levels of refraction at small reffraction angles. We are planning another series at a higher hohiraum density to test the limits of hohlraums heated using beams entering at the Nova incidence angles.

Author: J. D. Lindl

\section{$4 \omega$ Probing}

We used a simple analytic model to investigate the potential of ultraviolet interferometry for making plasma-density measurements of the channel conditions in hohlraum targets.

The model, described in Ref. 8, has been applied to simple disk targets. It assumes a one-dimensional plane plasma of length $L$ and density scale length $\ell$. It should provide a reasonable approximation of a cylindrical hohlraum if $\ell \ll R$, where $R$ is the cylinder radius, and if multidimensional effects can be neglected. By varying the model parameters, we can estimate the usefulness of an ultraviolet probe beam. We discuss here only those conclusions that differ from those in Ref 8.

The most important new conclusion is that probing the closed geometry produces an interferogram that is not Abel-invertible. Unlike the case of a simple disk target, where a two-dimensional (rotationally symmetric) density $n(r, z)$ can be unfolded from the data, the best that can be done for the hohlraum interior is a line-averaged measurement:

$\overline{\mathrm{n}(\mathrm{r}, \phi)}=\frac{1}{\mathrm{~L}} \int_{0}^{\mathrm{L}} \mathrm{n}(\mathrm{r}, \phi, \mathrm{z}) \mathrm{dz}$.

where $(r, \phi, z)$ is the usual cylindrical 
coordinate system, with $\mathrm{z}$ parallel to the hohlraum axis. The highest density resolved is approximately inversely proportional to the hohlraum length $\mathrm{L}$. The model indicates that a line-averaged density of $0.25 \times 10^{21}$ $\mathrm{cm}^{-3}$ will be measurable only under favorable conditions:

- Length $\mathrm{L}<1000 \mu \mathrm{m}$.

- Density scale length $\ell>100 \mu \mathrm{m}$, where $\ell$ $=(1 / \mathrm{ndn} / \mathrm{dr})^{-1}$.

- Density-surface velocities $\mathrm{V}<10^{7} \mathrm{~cm} / \mathrm{s}$.

- Probe pulse length $\tau<20 \mathrm{ps}$.

A second problem encountered in the

Fig. 4-23. Semienclosed targets simplify probing.

(a)

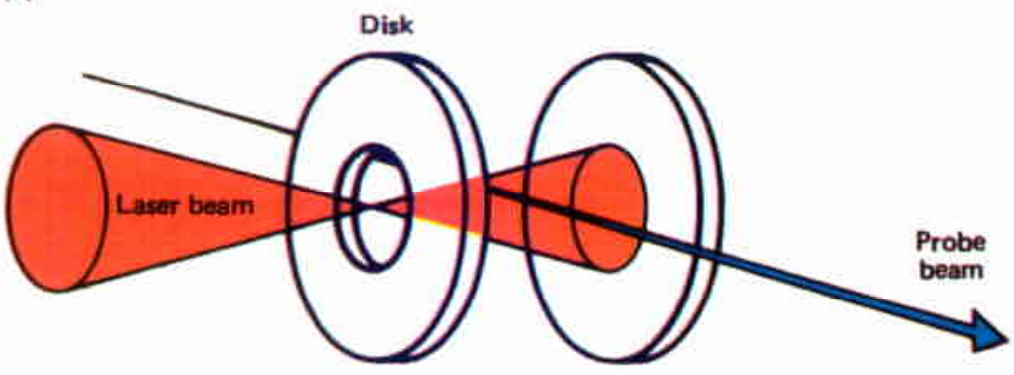

(b)
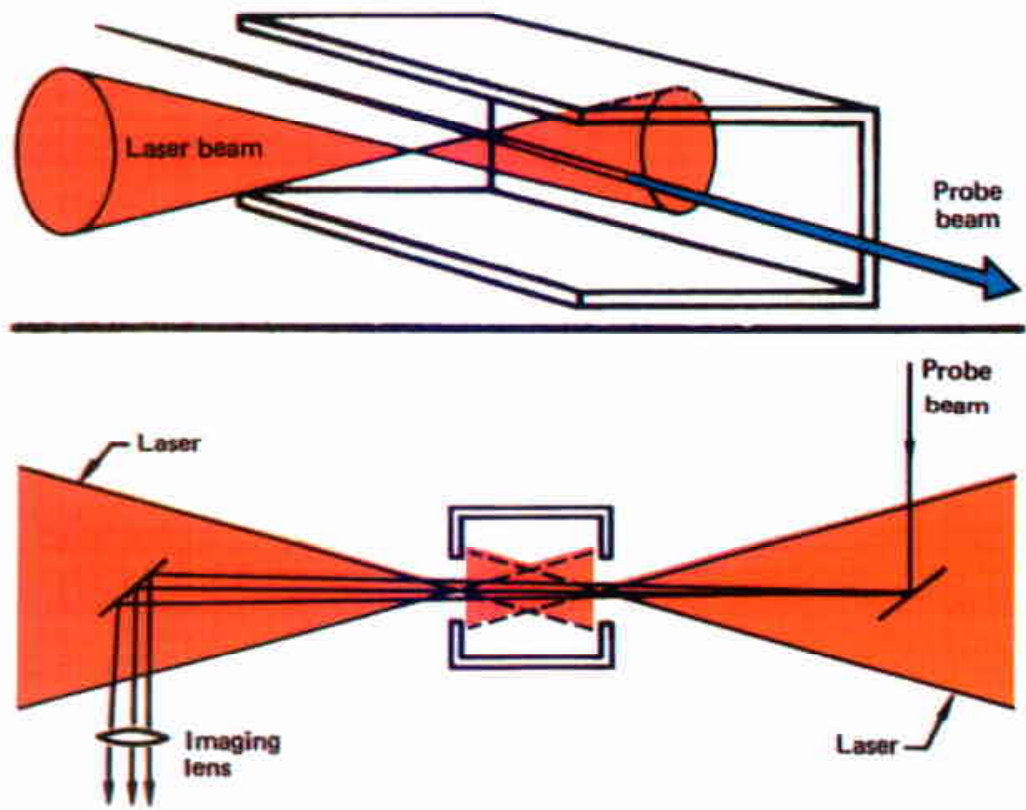

Fig. 4-24. Probing an unmodified

cylindrical hohlraum requires

inserting mirrors within laser

beams, as shown. obvious vacuum-to-plasma region always exists so that the "outermost" dark fringe clearly represents one half-wave of phase difference, the next outermost dark fringe comes from three half-waves of phase difference, etc. In the closed geometry, the vacuum reference is lost once the hohlraum density exceeds about $1 \times 10^{19} \mathrm{~cm}^{-3}$ (for L $=10^{3} \mu \mathrm{m}$ ) everywhere. This problem can be handled by making a series of interferograms, each separated by 100 to 200 ps, so that the first interferogram is made before the center density exceeds $10^{19} \mathrm{~cm}^{-3}$. The proper numbering of the fringes could then be done by extrapolation from the previous interferogram.

A holographic system is particularly well suited to record a series of time-multiplexed interferograms. An alternative procedure would be using a long-pulse probe and streak camera together. The streak record could follow the time evolution of the lowest-density fringes, since they impose the least demanding spatial resolution. The streak data could then be used to assign the lowest fringe numbers in a subsequent high-resolution interferogram.

Some of the problems mentioned above may be minimized by using semi-enclosed target geometries, as recently conducted by KMS Fusion." These are shown in Fig. 4-23(a) and (b). The target in Fig. 4-23(a) is obviously similar to a simple disk (as far as interferometric probing is concerned). The tanget in Fig. 4-23(b), though still only semi-enclosed, more closely resembles a hohlraum. Figure 4-24 shows the complex arrangement necessary to probe an unmodified cylindrical hohlraum.

A second use of interferometric probing is measuring the density and time history of plasma "plumes" emanating from the laser entrance or diagnostic holes. This geometry is Abel-invertible; all of the conclusions concerning disk targets apply, ${ }^{8}$ except that plasma profiles and velocities are difficult to calculate computationally. Therefore we cannot now estimate the highest observable density.

\section{Author: R. E. Turner}

Major Contributors: D. T. Attwood and J. M. Auerbach 
High-Density Studies

\section{Introduction}

We performed two significant sets of high-density experiments this year. First, Apollo double-shell fuel capsules in Caim hohlraums were irradiated with the full energy output of Shiva $(8$ to $10 \mathrm{~kJ})$. In three of seven experiments, fuel densities of 80 to $100 \times$ liquid-D-T density were measured by our neutron-activation diagnostic. The operating point of the capsules was near the threshold of the neutron-activation diagnostic as had been predicted by LASNEX computer simulations of the targets. Although higher thermal $x$-ray drive would have improved performance, this improvement was unattainable as the Shiva laser system was operated at its maximum output for these experiments.

Second, we initiated the first set of "Nova-like" implosion experiments. By Nova-like, we mean hohlraum conditions in which the drive-to-preheat ratio is comparable to conditions predicted for hohlraums designed for use with Nova. We call these experiments NPIRE (Nova precursor implosion research). In this set of experiments we tested fuel capsules in spherical hohlraums for the first time. These hohlraums produced higher drive and less preheat than cylindrical hohlraums of comparable size irradiated with the same laser energy. Target yield was predicted very accurately by computer simulations.

Authors: J. M. Auerbach, L. J. Suter, and Y. L. Pan

\section{Experiments with Apollo Intermediate-Density Targets}

We conducted a series of experiments to measure the performance of Apollo ${ }^{10}$ double-shell fusion targets in cylindrical hohiraums. In addition to characterizing the drive and preheat in the hohlraums, we successfully made three determinations of the compressed D-T-fuel density using our radiochemistry diagnostic. LASNEX preshot calculations and previous experiments indicated that Shiva can produce only marginal results with the Apollo capsule. Production of the required thermal $x$-ray drive for detectable neutron yields was obtainable only with small hohlraums, which resulted in high suprathermal electron levels and consequently degraded target performance because of preheat of the fuel and shell.

In this section we first describe the target and experiments in detail and then the computer simulations and analysis of target performance under experimental conditions.

Target Description. As shown in Fig. 4-25, the Apollo fuel capsule is distinguished from other high-density target designs in that the glass microsphere, which contains the fuel, and the ablator are separated by a $40-\mu \mathrm{m}$ void. The glass microballoon has a $140-\mu \mathrm{m}$ i.d. and a $20-\mu \mathrm{m}$-thick wall (which is four times thicker than the $5-\mu \mathrm{m}$-thick microballoon walls used in previous Cairn high-density experiments.) The thicker walls provide increased shielding for the fuel against hot electrons and $x$ rays. The optimum performance of the capsule will occur when the ablator is driven by thermal $x$ rays in an environment having no electron preheat. Computer simulations indicate that ablation of the $\mathrm{CH}$ shell accelerates the remaining mass to velocities exceeding $10^{7} \mathrm{~cm} / \mathrm{s}$. The $20-\mu \mathrm{m}$-thick glass pusher and D-T fuel are compressed by the momentum transfer from the ablator to pusher. The degree of compression critically depends on the preheating of the pusher by $x$ rays and hot electrons. If preheating is significant, the pusher decompresses and part of the ablator's mechanical energy is then

Fig, 4-25. Diagram of the Apollo fuel capsuie.

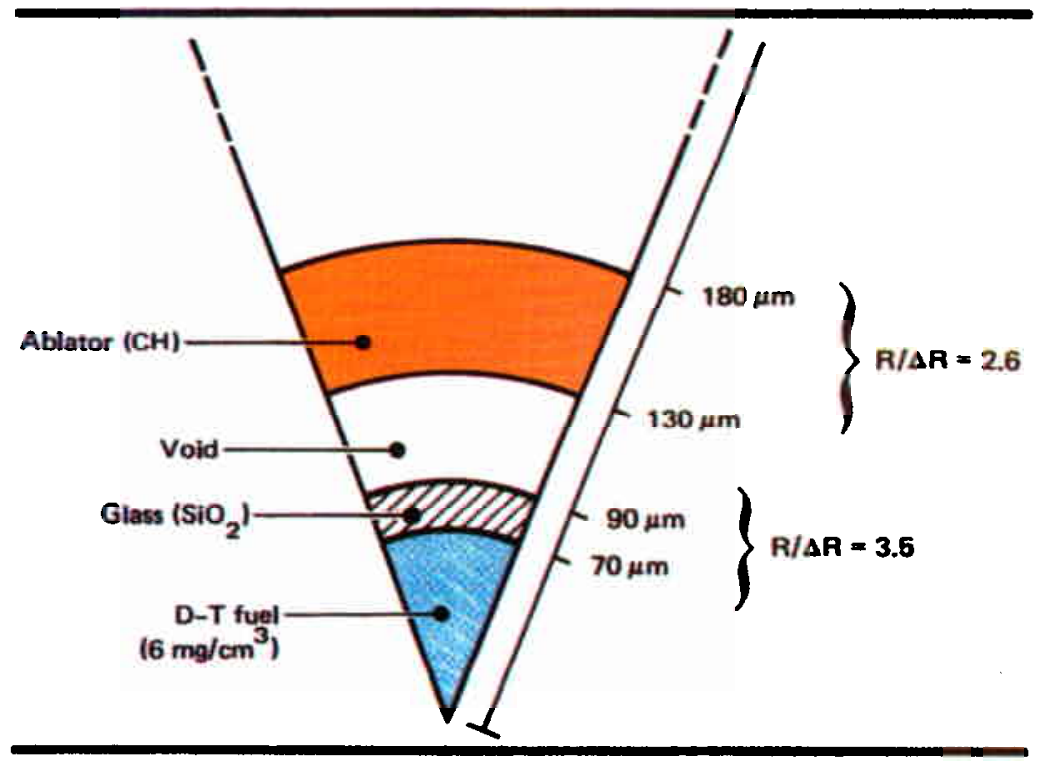




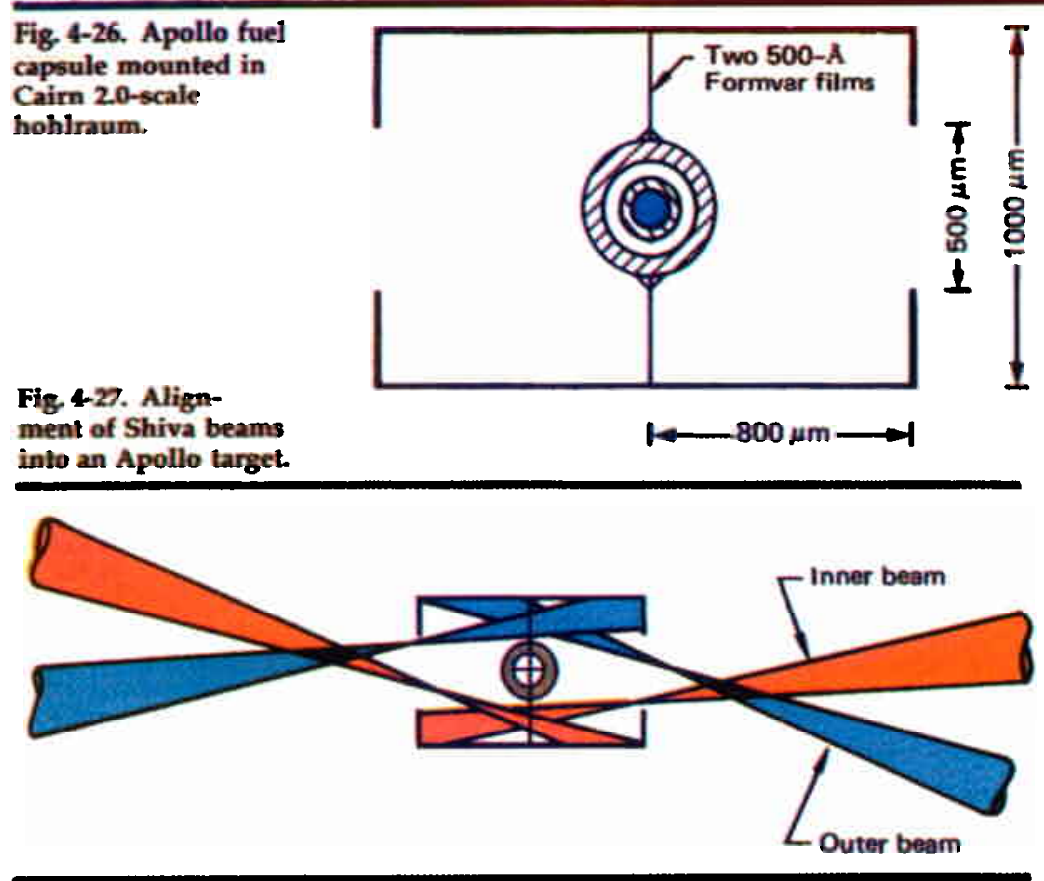

used to recompress the pusher. The effects of electron preheat on the pusher dynamics was discussed in Ref. 11.

In our experiments we mounted Apollo fuel capsules in hohlraums in the " $\mathrm{H}$ " configuration: the capsule was supported by only a set of thin membranes (Fig. 4-26). The figure shows a 20-scale Caim (1000- $\mu \mathrm{m}$ diam and $1600-\mu \mathrm{m}$ long); both 2.0 and 2.5-scale Cairns (1250- $\mu \mathrm{m}$ diam and 2000- $\mu \mathrm{m}$ long) were used in the experiments.

Laser Illumination of Hohlraums. The hohlraums were irradiated by the Shiva laser system with 8-kJ/600-ps FWHM 1.06- $\mu \mathrm{m}$ laser pulses. Figure 4-27 illustrates the alignment of the 20 Shiva beams into the hohlraum, showing two beams from each cluster. One of the ray cones represents an "outer" cluster beam, the median ray, which is tilted at an angle of $17.6^{\circ}$ relative to the vertical target-chamber axis. The other ray cone represents an "inner" cluster beam, the median ray, which is tilted at an angle of $9.7^{\circ}$ relative to the target-chamber axis. Each cone corresponds to that of an $\mathrm{f} / 6$ lens (cone angle $9.8^{\circ}$ ). The remaining eight beams in each cluster are distributed azimuthally around the chamber axis. Adjacent inner- and outer-beam axes are separated by an angle of $36^{\circ}$. The beams in each cluster are positioned so that they focus at the laser entrance hole of the hohlraum, miss the $\mathrm{CH}$ ablator of the fuel capsule, and irradiate the two $500-\dot{A}$
Formvar films supporting the fuel capsule. Using a 400-J/600-ps laser pulse from each beam, the calculated first-bounce intensity on the film in a 2.0-scale hohlraum is $3.6 \times$ $10^{15} \mathrm{~W} / \mathrm{cm}^{2}$, while for the same laser conditions the first-bounce intensity on the film in a 2.5-scale hohiraum is $2.3 \times 10^{15}$ $W / \mathrm{cm}^{2}$. On irradiation the film forms a low-Z plasma that, on interaction with the laser beams, can produce suprathermal electrons by the nonlinear plasma processes of stimulated Raman scattering and two plasmon decay. We describe the Raman scattered light observed during the Apollo experiments in a following section.

Summary of Experiments. We carried out the Apollo experiments at various times between October 1979 and August 1980, and Table 4-10 summarizes the 13 target experiments conducted. Three experiments measured thermal $x$-ray drive, high-energy $x$ rays, and neutron yield simultaneously; three experiments measured the collection efficiency of the radiochemistry catcher for an Apollo fuel capsule (providing two good results); and eight experiments measured the $\rho \Delta R$ of the glass pusher at peak thermonuclear burn time to infer fuel density (providing three good results).

Drive and Preheat Measurements. The Apollo fuel capsule was designed for thermal $x$-ray drive in the range of $\geq 150 \mathrm{eV}$ to provide yields of $10^{6}$ neutrons and a pusher areal density $\rho \Delta R$ of $0.1 \mathrm{~g} / \mathrm{cm}^{2}$. At the time of the initial experiments, we did not have verified scaling laws to predict drive for a given Caim hohlraum size, laser energy, and laser pulse width. We therefore used computer simulations to choose an initial hohlraum size: a 2.5-scale hohlraum. We conducted three initial experiments (shots 89101105,80031702 , and 80031804 in Table 4-10) to ascertain that we had achieved the required drive and that the fuel capsules were producing yields in excess of $10^{6}$ neutrons, which is the threshold required for a $\rho \Delta R$ measurement by radiochemistry. As shown in Table 4-10, the values of drive $\left(T_{R}\right)$ were at or below the limit of the range of desired values. In addition, only two experiments (shots 80031804 and 80032605 ) produced more than $10^{6}$ neutrons.

Based on the results of these three experiments, we decided to use 2.0-scale Caim hohiraums for all subsequent Apollo experiments to maximize the drive. Recently 


\begin{tabular}{|c|c|c|c|c|c|}
\hline $\begin{array}{l}\text { Shot } \\
\text { number }\end{array}$ & $\begin{array}{l}\text { Caim } \\
\text { hohiraum scale } \\
\text { (fuel capoule) }\end{array}$ & $\begin{array}{l}\text { Purp } \\
\text { expe }\end{array}$ & $\begin{array}{ll}\text { Laser pulse } & \text { Neutron } \\
\text { (k) } / \text { ns }) & \left(\times 10^{6}\right)\end{array}$ & $\begin{array}{l}\text { Pusher } \rho^{A R} \\
\left.\text { (b/cm })^{2}\right) \\
\text { or catcher } \\
\text { efficiency } \\
(\%)\end{array}$ & $\begin{array}{l}\text { Drive, } T_{R} \\
\text { (ev) }\end{array}$ \\
\hline 8910 & Apollo 19) & $\begin{array}{l}\text { Mea } \\
\text { driv } \\
\text { neu }\end{array}$ & 1 & & \\
\hline 80031702 & $\begin{array}{l}\text { 2.5-H } \\
\text { (Apollo 12) }\end{array}$ & $\begin{array}{l}\text { Me } \\
\text { dri } \\
\text { net }\end{array}$ & $8.32 / 600 \quad 0.2$ & & E. \\
\hline 80031804 & $\begin{array}{l}\text { 5.H } \\
\text { ipollo }\end{array}$ & $\begin{array}{l}\text { Mea } \\
\text { driv } \\
\text { neut }\end{array}$ & $66 / 600 \quad 1.6 \pm 02$ & & Q \\
\hline 80032605 & A-H & $\begin{array}{l}\text { Measurement } \\
\text { pusher pAR } \\
\text { neutron yield }\end{array}$ & $8.25 / 600 \quad 1.9 \pm 0.4$ & Pusher of & \\
\hline 800 & $\begin{array}{l}20-\mathrm{H} \\
\text { (Apollo 16) }\end{array}$ & $\begin{array}{l}\text { Catc } \\
\text { effic } \\
\text { mea }\end{array}$ & $0 \quad 0.50 \pm 0.42$ & $\begin{array}{l}\text { Catcher } \\
25.8 \%\end{array}$ & \\
\hline 80040906 & $\begin{array}{l}2.0-H \\
\text { (Apollo 15) }\end{array}$ & $\begin{array}{l}\text { vent } \\
\text { ield }\end{array}$ & $7.08 / 600 \quad 0.32 \pm 0.13$ & Below : & \\
\hline 80041005 & $\begin{array}{l}\text { 25-H } \\
\text { (Apollo 18) }\end{array}$ & & $8.83 / 600 \quad 0.74$ & & \\
\hline 80041010 & $\begin{array}{l}\text { 25-H } \\
\text { (Apollo 19) }\end{array}$ & & $8.71 / 600 \quad 0.34 \pm 0.13$ & Below b & \\
\hline 80072408 & $\begin{array}{l}20-\mathrm{H} \\
\text { (Apollo 24) }\end{array}$ & $\begin{array}{l}\mathrm{C} \\
\mathrm{eff} \\
\mathrm{mi}\end{array}$ & $7.31 / 600 \quad 0.53 \pm 0.20$ & $\begin{array}{l}\text { Equ } \\
\text { no }\end{array}$ & \\
\hline 8007 & $\begin{array}{l}20-\mathrm{H} \\
\text { (Apollo 16) }\end{array}$ & ment & $8.17 / 600 \quad 0.82 \pm 0.28$ & $\begin{array}{l}\text { Catcher } \\
26.1 \%\end{array}$ & \\
\hline 80080104 & $\begin{array}{l}\text { 20-H } \\
\text { (Apollo 23) }\end{array}$ & & $\begin{array}{l}8 \text { (est) } \quad 0.42 \pm 0.05 \\
1600\end{array}$ & Below background & \\
\hline 800 & $\begin{array}{l}20-\mathrm{H} \\
\text { (Apollo 20) }\end{array}$ & $\begin{array}{l}\text { Me } \\
\text { pus } \\
\text { neu }\end{array}$ & $0.88 \pm 0.41$ & & \\
\hline 800 & $\begin{array}{l}\text { 20-H } \\
\text { (Apollo 21) }\end{array}$ & $\begin{array}{l}\text { Me } \\
\text { pus } \\
\text { net }\end{array}$ & $9.24 / 600 \quad 1.7 \pm 0.4$ & $\begin{array}{l}\rho \Delta R\left(\mathrm{~g} / \mathrm{cm}^{2}\right) \\
0.035 \pm 0.02 \mathrm{~J}\end{array}$ & \\
\hline 80082006 & $\begin{array}{l}20-\mathrm{H} \\
\text { (Apollo 22) }\end{array}$ & Meas & $851 / 600 \quad 0.53 \neq 0.26$ & No paR & \\
\hline
\end{tabular}

derived scaling laws ${ }^{12}$ indicate that the decrease in scale size will increase the drive by the ratio $(2.5 / 2)^{2}=1.56$ and that the hot-electron preheat increases by the ratio $(25 / 3)^{3}=1.95$.

Experimental Results. Figure 4-28 shows the measured neutron yield as a function of laser energy for all targets. we could make no correlation of yield and incident laser energy. The scatter is partially attributed to misalignment of the laser beams into the laser entrance hole of the hohlraum. We discovered this by examining $x$-ray micrographs viewing the upper end cap of the hohlraum. Several micrographs showed bright spots on the lip of the hohlraum, which indicated that a portion of one or more beams struck the end cap, meaning that less energy entered the hohiraum than expected. In addition, the scatter could have 
Table 4-11. Density measurement data very close to lower limit of detectability of radiochemistry system.

Table 4-12. Fuel densities at peak thermonuclear burn derived from simple model.

Fig. 4-28. Neutron yield as function of incident energy for Apollo experiments.

High-Density Studies

\begin{tabular}{ccccc}
\hline $\begin{array}{c}\text { Shot } \\
\text { number }\end{array}$ & $\begin{array}{c}\text { Neutron yield } \\
\left(\mathrm{N}_{\mathrm{y}}\right)\end{array}$ & Pusher $\mathrm{p \Delta R}$ & $\left(\mathrm{N}_{\mathrm{y}}\right)(\mathrm{a \Delta R})$ & $\frac{(\mathrm{Ny})(\mathrm{a} \Delta \mathrm{R})^{2}}{5.06 \times 10^{4}}$ \\
\hline 80032605 & $(1.9 \pm 0.4) \times 10^{6}$ & $0.093 \pm 0.039$ & $17.7 \times 10^{4}$ & 3.50 \\
80082604 & $(8.8 \pm 4.1) \times 10^{5}$ & $0.083 \pm 0.062$ & $7.3 \times 10^{4}$ & 1.44 \\
80082703 & $(1.7 \pm 0.4) \times 10^{6}$ & $0.035 \pm 0.027$ & $5.95 \times 10^{4}$ & 1.18 \\
\hline
\end{tabular}

$5.06 \times 10^{4}$ is threshold value of $\left(N_{y}\right)(0 \Delta R)$ for radiochemistry system.

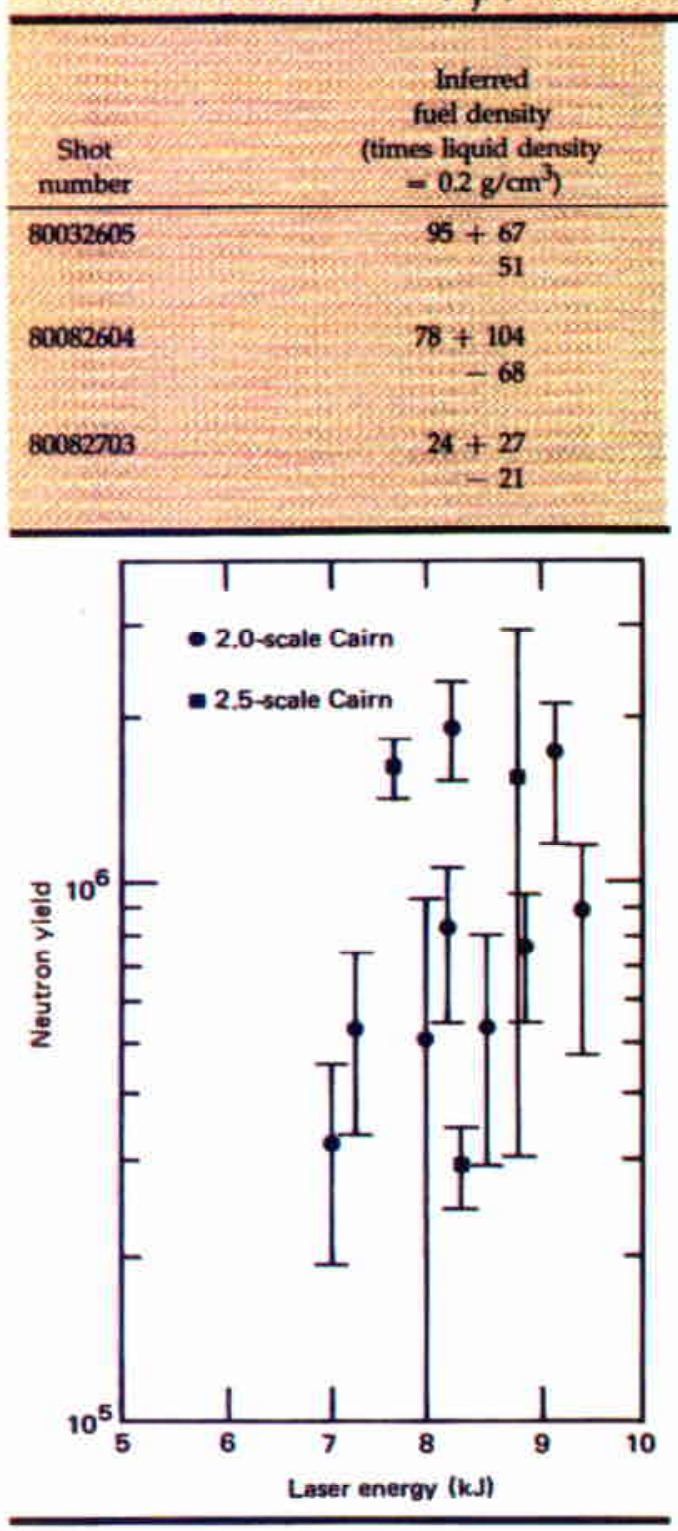

resulted from nonuniform preheat, nonuniform drive, or nonuniform implosion of the $\mathrm{CH}$ ablator. The contribution of each of these factors cannot at present be determined experimentally.

Pusher $\rho \Delta R$ Measurements. We measured the pusher $\rho \Delta R$ using the neutronactivation (radiochemistry) diagnostic. References 13 and 14 describe this system and the mechanics of the measurement in detail. Before making the $\rho \Delta R$ measurements on the Apollo capsules, we had to measure the efficiency of the radiochemistry catcher for an Apollo target. There was no prior reason to reason that the catcher efficiency would be the same as that for a $140-\mu \mathrm{m}$ radius with a 5- $\mu \mathrm{m}$-thick glass wall and a 15- $\mu \mathrm{m}$-thick $\mathrm{CH}$ ablator ${ }^{15}$ in a Cairn target (62\% efficient) or the same as the value of a ball-on-stalk target ${ }^{16}$ ( $55 \%$ efficient). Three catcher-efficiency tests were made with Apollo targets having activated glass pushers. Two of the three experiments (shots 80040414, 80072408, and 80072513 in Table 4-10) produced catcher efficiency data. The third had no data because of a mechanical failure of the collector mechanism. We derived an average value of $26 \pm 3 \%$ from the data. This value is valid only for the conical catcher lined with a titanium foil and placed $1 \mathrm{~cm}$ from the target.

Of the eight pusher $\rho \Delta R$ experiments, only three Apollo capsules (shots 80032505 , 80082604 , and 80082703 ) produced a sufficient number of activated silicon atoms for the radiochemistry detection system to produce a signal above background. These three values were near the lower limit of detection, which can be expressed as the following relationship between yield $N_{y}$ and pusher $\rho \Delta R$ :

$N_{y}(\rho \Delta R) \geq 4.6 \times 10^{4}$.

Table 4-11 shows the calculated values of $N_{y} \rho \Delta R$ for the three experiments. Because our measurements were moderately above the lower operating limit of the system for only one of the three experiments, the resulting measurements have large error ranges. To correct this situation, future experiments will require much higher yields, which can be accomplished by using larger hohlraums and larger energies to decrease the preheat-to-drive ratio. Shorterwavelength lasers will also improve this ratio.

D-T fuel densities are inferred from the pusher $\rho \Delta R$ using two methods:

- LASNEX computer simulation of fuelpusher dynamics.

- A simple model assuming an isothermal 
isobaric pusher-fuel region ${ }^{17}$ in which we assume that the pusher and fuel are modeled by an ideal gas.

In either case the pusher $\rho \Delta R$ used as input to the calculations corresponds to the time of peak thermonuclear burn, which is not necessarily the time of peak fuel density. An estimate of the delay between the two times and difference between the two peak quantities can only be estimated from computer simulations (see below). Application of the simple model for an Apollo pusher (70- $\mu \mathrm{m}$ inner radius, $20-\mu \mathrm{m}$-thick wall, and an ionization state for the $\mathrm{SiO}_{2}$ of $\mathrm{Z}=7$ ) yields the following relationship between fuel density $\rho_{i}$ and pusher $\rho \Delta R$ at peak burn time.

$\rho_{\mathrm{f}}=675(\rho \Delta \mathrm{R}) 3 / 2$.

Table 4-12 presents the calculated fuel-density values for the three acceptable $\rho \Delta \mathrm{R}$ measurements in the sequence. The experiments indicate, even with the large error, that fuel densities of the order of $100 \times$ liquid density have been attained.

Author: J. M. Auerbach

Major Contributors: S. M. Lane and K. G. Tirsell

\section{Analysis of Apollo Intermediate-Density Targets}

The preshot Shiva-scale intermediatedensity Apollo target design study, first discussed in 1978, has been presented elsewhere. ${ }^{18,19}$ Details of target experiments are given in the preceding section. We furnish here a preliminary analysis of the experimental data and compare these results with the preshot one-dimensional LASNEX simulation predictions. We emphasize at the outset that the available experimental data are exceedingly sparse and that some of the tentative conclusions are based on comparing results of single shots. More supporting experiments will be required to change some of these preliminary inferences to firm conclusions.

We find that the intermediate-density Apollo target performance substantially agrees with the LASNEX predictions. ${ }^{19}$ The magnitude of the experimental neutron yields gives unambiguous evidence that most, if not all, of the target drive was provided by $x$ rays. On the basis of glass $p \Delta R$ and $x$-ray temperature data, we infer that the suprathermal electron preheat of the glass tamper is lower than expected from preshot one-dimensional LASNEX calculations. A comparison between the neutron yield, glass $\rho \Delta R$, and preshot LASNEX simulation results indicates that peak fuel densities of about $20 \mathrm{~g} / \mathrm{cm}^{3}$ (100X liquid density) has been achieved and that neutron-yield degradation from fuel-tamper mixing effects is not very significant. When the size of the glass tamper was reduced in the Apollo target, preshot LASNEX simulations predicted increased fuel compression and reduced neutron yield. Experimental observations were consistent with this prediction. Furthermore, evidence suggests that target-fabrication imperfections and/or radiation-temperature (drive) asymmetry can cause large reductions in the neutron yield. We elaborate on these statements below.

Table 4-13 contains most of the relevant data required for the remaining discussion. These target shots are a subset of those provided in Table 4-10 and represent those experiments where the recorded neutronactivation counts were at least three standard deviations above background and where the uncertainties of the neutron-yield
Table 4-13. Selected parameters for Apollo target experiments.

\begin{tabular}{|c|c|c|c|c|c|c|c|c|c|c|c|c|}
\hline \multirow[b]{3}{*}{$\begin{array}{c}\text { Shot } \\
\text { number? }\end{array}$} & \multirow{2}{*}{\multicolumn{2}{|c|}{ Glass tamper }} & \multirow{3}{*}{$\begin{array}{c}\text { D-T } \\
\text { fill } \\
\left(\mathrm{mg} / \mathrm{cm}^{3}\right)\end{array}$} & \multicolumn{4}{|c|}{$\mathrm{CH}$ shell } & \multirow{3}{*}{$\begin{array}{l}\text { Shell } \\
\text { separa- } \\
\text { ation } \\
\text { (jm) }\end{array}$} & \multirow{2}{*}{\multicolumn{2}{|c|}{ Laser energy }} & \multirow{3}{*}{$\begin{array}{c}\text { Radiation } \\
\text { temperature } \\
(\mathrm{eV}) \\
\text { or glass } \rho \Delta R \\
\left(\mathrm{~g} / \mathrm{cm}^{2}\right)\end{array}$} & \multirow{3}{*}{$\begin{array}{c}\text { Neutron } \\
\text { yield } \\
\left(\times 10^{5}\right)\end{array}$} \\
\hline & & & & \multicolumn{2}{|c|}{ Hemisphere 1} & \multicolumn{2}{|c|}{ Hemisphere 2} & & & & & \\
\hline & $\begin{array}{l}\text { i.d. } \\
\text { (um) }\end{array}$ & $\begin{array}{l}\text { Thick } \\
\text { (um) }\end{array}$ & & $\begin{array}{l}\text { i.d. } \\
\text { (um) }\end{array}$ & $\begin{array}{l}\text { Thick } \\
\text { (um) }\end{array}$ & $\begin{array}{l}\text { id } \\
\text { (jm) }\end{array}$ & $\begin{array}{l}\text { Thick } \\
(\mu \mathrm{m})\end{array}$ & & $\frac{E_{B}}{E_{T}}$ & $\frac{E_{H}}{E_{L}}$ & & \\
\hline 31702 & 136.6 & 19.0 & 6 & $256 \pm 2$ & $49 \pm 1$ & $258 \pm 2$ & $45 \pm 1$ & 41.2 & 1.08 & 1.84 & $156 \pm 10$ & $2.9 \pm 0.5$ \\
\hline 31804 & 129 & 21.0 & 6 & $262 \pm 2$ & $47 \pm 1$ & $258 \pm 2$ & $49 \pm 1$ & 44.5 & 0,97 & 1.61 & $160 \pm 10$ & $16 \pm 2.0$ \\
\hline 32605 & 145.4 & 178 & 6 & $274 \pm 2$ & $44 \pm 1$ & $274 \pm 2$ & $44 \pm 1$ & 465 & 1.09 & 1.77 & $0.093 \pm 0.039$ & $19 \pm 4.0$ \\
\hline 82604 & 136 & 20.0 & 6 & $256 \pm 2$ & $52 \pm 1$ & $259 \pm 2$ & $51 \pm 1$ & 40.8 & 1.02 & 1.31 & $0.083 \pm 0.062$ & $8.8 \pm 4.1$ \\
\hline 82703 & 152.8 & 17.6 & 6 & $256 \pm 2$ & $51 \pm 1$ & $258 \pm 2$ & $50 \pm 1$ & 345 & 1.01 & 1.32 & $0.035 \pm 0.027$ & $17 \pm 4.0$ \\
\hline
\end{tabular}

"First three digits $(800)$ of shot numbers have been dropped. 
data were not overly large. It is difficult, and probably impossible, to derive reliable estimates of the radiation-temperature (drive) asymmetries in these experiments. However, the ratio of the incident laser energies of the bottom 10 beams to the top 10 beams $\left(\mathrm{E}_{\mathrm{B}} / \mathrm{E}_{\mathrm{T}}\right)$ may be a crude measure of the difference in drives experienced by the top and bottom hemispheres. The incident laser-energy ratio of the most intense to the weakest beam $\left(E_{H} / E_{1}\right)$ may give a weak signature of the local variations in the preheat and/or drive.

The main purpose of the first two experiments (shot numbers 31702 and 31804) was to measure the hohlraum radiation temperature. Consequently, other factors such as neutron yield and target-fabrication imperfections were of secondary consideration. The time-integrated low-energy $x$-ray spectra obtained by the Dante systems for these two experiments were nearly identical, but the gold hohlraum in shot 31702 had a slightly larger diagnostic hole and thus a lower measured radiation temperature. However, within the experimental uncertainties these two tangets achieved the same $x$-ray temperature and this negligible difference in drive cannot be the main source of the measured neutron yield discrepancy. The glass tamper in shot 31702 had a larger inside diameter, but $\mathbf{2 . 8 \%}$ less mass. If other conditions were identical, it should have had a higher instead of a lower neutron yield. But, note that the differences in the outer and inner radii of the two $\mathrm{CH}$ hemispheres used in 31702 were 3 and $1 \mu \mathrm{m}$, respectively. The two $\mathrm{CH}$ hemispheres used in 31804 had the same outer radii and a

Fig. 4-29. Temporal shapes of $x$ rays with energies $\geq 30 \mathrm{keV}$ and scattered 1.06-um laser radiation for Caira carget.

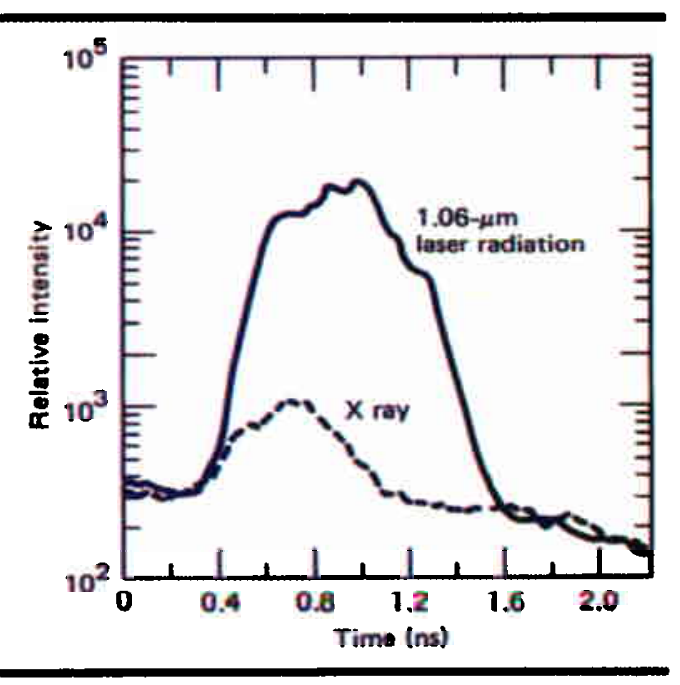

$2 \mu \mathrm{m}$ difference in inner radii. We speculate that the factor of 5 discrepancy in the observed neutron yield was due mainly to the target fabrication variations. But the slight differences in the incident laser energy imbalances (drive and preheat?) may also be contributing factors.

The only significant differences between the targets used in the last two experiments (shot numbers 82604 and 82703 ) were the size and thickness of the glass tampers. Fortuitously, the difference in the glass-tamper masses was small (only $4.8 \%$ ) and should not affect our conclusion. These data suggest that higher compression (inferred from the higher glass $\rho \Delta R$ ) and lower neutron yield can be obtained by reducing the glass-tamper size while keeping other factors in the target constant. This trend is consistent with the predictions of the preshot LASNEX simulations. ${ }^{19}$ Additional calculations are required to obtain a more detailed comparison.

Preshot one-dimensional LASNEX simulations showed that the maximum neutron yield for a suprathermal electron-driven Apollo target (without $x$-ray drive) was $2 \times$ $10^{5}$ (Table 2-5, Ref. 19). The glass $\rho \Delta \mathrm{R}$ in this case was $7 \times 10^{-3} \mathrm{~g} / \mathrm{cm}^{2}$. We recall that two- and three-dimensional effects can substantially reduce the calculated one-dimensional neutron yields. For exploding-pusher targets, two-dimensional simulated or experimental neutron yields were typically about an order of magnitude lower than those obtained from preshot one-dimensional calculations. Thus, one should only expect an experimental neutron yield of about $2 \times 10^{4}$ if these Apollo targets were driven by suprathermal electrons. The fact that the experimental values are 10 to 100 times higher is unambiguous evidence that the dominant driving mechanism is $x$ rays. The fact that the measured glass $\rho \Delta R$ was an order of magnitude higher offers additional support for this conclusion.

Near the beginning of the Apollo experimental schedule, we repeated some of the preshot one-dimensional LASNEX simulations discussed in Ref. 19 using more appropriate parameters. The laser-pulse duration (and that of the radiation temperature source) was reduced to $0.6 \mathrm{~ns}$ to correspond to the experimental conditions. Preliminary streak-camera data (Fig. 4-29) indicated that 
$x$ rays having energies $\geq 30 \mathrm{keV}$ (and by inference, the suprathermal electrons) were produced during the first half of the laser pulse in Cairn targets. ${ }^{20}$ Consequently, we imposed the production of suprathermal electrons with a constant temperature $T_{\text {hot }}$ of $50 \mathrm{keV}$ during only the first half of the laser pulse in these computer calculations. The PBINTR radiation transport and NLTE-atomic physics on the gold case were used. Reference 19 contains more details of the model.

Figure 4-30 shows the results of the one-dimensional calculation when $\mathrm{f}_{\text {how }}$ the fraction of the incident laser energy placed into suprathermal electrons, was assumed to be $30 \%$ for various peak radiation temperatures. Even at a peak driving temperature of $175 \mathrm{eV}$ (well above the measured hohlraum condition) the glass $\rho \Delta R$ at the time of peak D-T burn is only about 0.07 $\mathrm{g} / \mathrm{cm}^{2}$. We chose to make the comparison at peak D-T burn because neutron activation samples the glass tamper $\rho \Delta R$ at this time. The peak compression occurs after this time and the maximum fuel density can be extracted only from computer simulations. Although the calculated tamper $\rho \Delta \mathrm{R}$ values are within the experimental uncertainties of the measured values, they are always lower than the central values obtained for shots 32605 and 82604 . This strong bias places justifiable suspicion on the validity of some of the assumptions used in the simulations.

In Fig. 4-31 we show the results of LASNEX simulations when the $\mathrm{f}_{\text {hot }}$ value is varied for a fixed FDS source having a peak $x$-ray temperature of $160 \mathrm{eV}$. The glass tamper $\rho \Delta R$ and neutron yield are very sensitive to the assumed suprathermal-electron preheat. To obtain the measured glass $\rho \Delta R$ value of about 0.08 to $0.09 \mathrm{~g} / \mathrm{cm}^{2}, \mathrm{f}_{\text {hot }}$ must be lower than the value obtained from FFLEX measurements (30\%) and/or fewer suprathermal electrons should be transported to preheat the glass tamper. The following evidence lends support to this conclusion.

The suprathermal electron flux is deduced from the number of high-energy $x$ rays detected by the FFLEX system and the electron bremsstrahlung cross section on gold. Different unfolding techniques have given values for suprathermal-electron flux that vary by a factor of 2 (Ref. 21). Thus, the measured suprathermal-electron flux is uncertain to this factor. LASNEX simulations of laser experiments using layered-slab targets indicated that the calculation produced too many suprathermal electrons and allowed too many of them to penetrate deeply into the target. ${ }^{22}$ Strong suprathermal-electron transport inhibition and a reduction in the suprathermalelectron production had to be invoked to obtain approximate agreement between calculated and experimental data. Recent suprathermal $x$-ray streak-camera data (Fig. 4-32) show that the temporal profile of the high-energy $x$ rays produced in the Apollo target follows the shape of the incident laser pulse and is different from that obtained for the Cairn target (Fig. 4-29). ${ }^{20}$ We do not yet know the reason for the observed difference, but this change should delay the onset of the suprathermal-electron preheat of the glass tamper and reduce its deleterious effects on the target implosion.
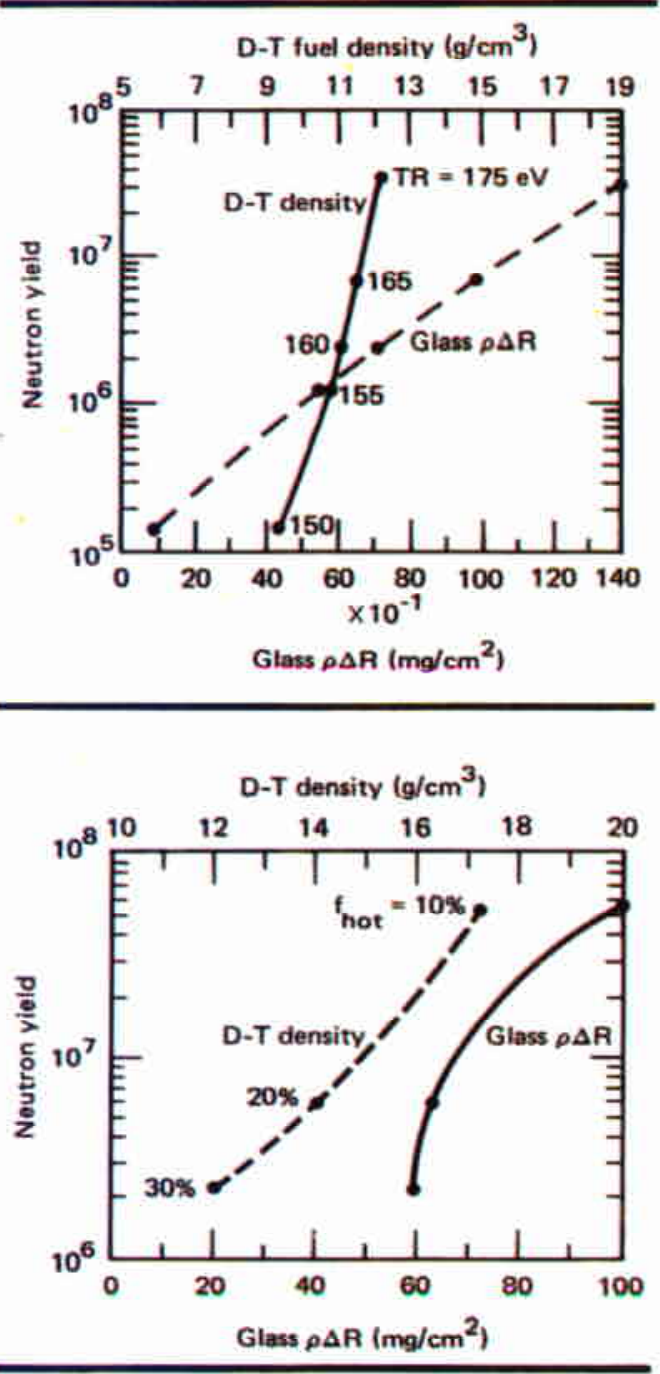

Fig. 4-30. Calculated fuel density, glass $\rho \Delta R$, and neutron yield at time of peak $D$-T burn; $f_{\text {hot }}$ is fixed at $30 \%$ and peak channel-radiation temperature is varied.

Fig. 4-31. Calculated fuel density, glass $\rho \Delta \mathbf{R}$, and neutron yield at time of peak D-T burn; $\mathrm{f}_{\text {hot }}$ is allowed to vary and peak channelradiation temperature is fixed at $160 \mathrm{eV}$. 
Other effects that may reduce the suprathermal-electron preheat are related to the inherent errors of attempting to simulate two- and three-dimensional problems with one-dimensional calculations. For example, suprathermal-electron flux may not

Fig. 4-32. Temporal shapes of $x$ rays with energies $\geq 30 \mathrm{keV}$ and scattered 1.06- $\mathrm{mm}$ laser radiation for Apollo target.

Table 4-14. Calculated fuel density, glass $\rho \Delta R$, and neutron yield at different implosion times.

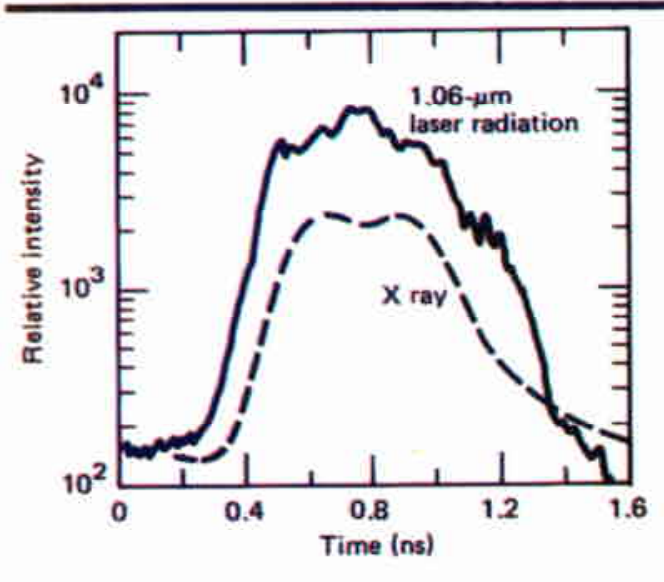

\begin{tabular}{lccc} 
& $\begin{array}{c}\text { Fuel density } \\
\left(\mathrm{g} / \mathrm{cm}^{3}\right)\end{array}$ & $\begin{array}{c}\text { Glass } \rho \Delta \mathrm{R} \\
\left(\mathrm{g} / \mathrm{cm}^{2}\right)\end{array}$ & $\begin{array}{c}\text { Neutron } \\
\text { yield }\end{array}$ \\
\hline At mix time & 4 & 0.055 & $2 \times 10^{5}$ \\
At peak burn & 16 & 0.097 & $2 \times 10^{7}$ \\
At peak compression & 20 & 0.11 & $5 \times 10^{7}$ \\
\hline${ }^{2} \mathrm{f}_{\text {hot }}=10 \%$ and pecik radiation temperature $=150 \mathrm{eV}$ & \\
\hline
\end{tabular}

Fig. 4-33. Calcu-

lated radii vs time

plot for some Apollo

target components.

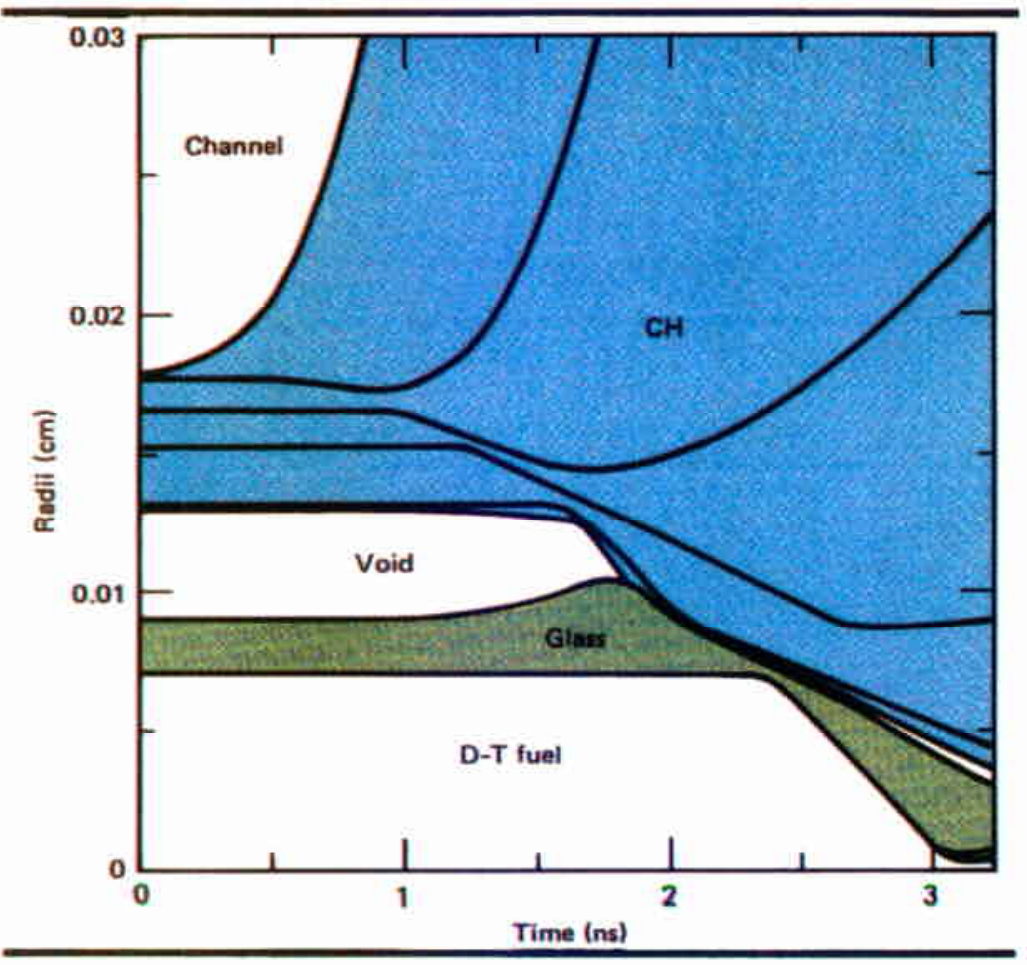

be isotropic in the hohlraum, or laser intensity on the surfaces of the case and outer shell may not be uniform (as is assumed). Our suggested laser-beam-alignment scheme in these experiments avoided, as much as possible, laser irradiation of the $\mathrm{CH}$ outer shell. We also excluded the possible beneficial effects of vacuum insulation in these one-dimensional simulations when the mass of the webs used to support the fuel capsule was distributed uniformly in the void between the two shells. Our original LASNEX model was too pessimistic. Thus suprathermal-electron preheat of the glass tamper should be reduced by a factor of 2 to 3 .

The $\mathrm{CH}$ hemispheres in shot 32605 were perfectly matched. This experiment also produced the highest glass- $\rho \Delta R$ and neutron-yield values. However, the neutron yield is not significantly above that obtained in shot 31804. Fortuitously, the glass capsules used in these two targets had the same mass and the capsule radius in 32605 was larger. Thus, we would have expected a much larger difference in the neutron yields. The larger incident-laser-energy imbalances may be part of the reason for the lower-than-anticipated yield. But polar $\mathrm{x}$-ray microscope data for shot 32605 indicated that more than 2 of the upper 10 laser beams missed the laser entrance aperture and hit the outer face of the gold case. ${ }^{23}$ Consequently, we believe that the target 32605 had a channel-radiation temperature below $160 \mathrm{eV}$ and that this lower $\mathrm{x}$-ray drive was the main reason for the reduction in the expected neutron yield. Table 4-14 contains the calculated fuel density, glass $\rho \Delta \mathrm{R}$, and neutron yield at various implosion times for an Apollo target subjected to a $150-\mathrm{eV}$ peak radiation temperature FDS source and an $\mathrm{f}_{\text {hot }}$ of $10 \%$. Figure 4-33 shows the radii vs time plot for some of the target components. The calculated glass $\rho \Delta R$ at the time of peak D-T bum is in excellent agreement with the measured value. There is a factor of 10 difference in the calculated and experimental neutron yield, but as explained above, this discrepancy was anticipated and can be attributed to two- and three-dimensional effects. The neutron yield at mix time is substantially lower than that attained at the time of peak D-T burn. This suggests that neutron-yield degradation from tamper-fuel mixing effect is not very 
significant. The peak fuel density reached $20 \mathrm{~g} / \mathrm{cm}^{3}$ (100X liquid density).

The success of the Shiva-scale intermediate-density target demonstrates that LASNEX can be used to predict the performance of complex targets.

Two-dimensional simulations will be required to obtain a more detailed comparison between the calculated and experimental data.

Author: Y. L. Pan

\section{NPIRE Experiments}

Background. Data from hohlraum-scaling and wavelength-scaling experiments indicate that large Nova-type hohlraums may be characterized as having a radiation temperature of about $160 \mathrm{eV}$ and converting less than $10 \%$ of the laser energy into suprathermal electrons $\left(f_{\text {hot }} \leq \mathbf{0 . 1}\right)$.

To advance our capability to model, design, and diagnose fusion targets operating in this drive and preheat regime, we performed an experimental sequence with Nova-like targets on the Shiva laser system. This series is called Nova precursor implosion research (NPIRE).

In the NPIRE series we irradiated spherical hohlraums for the first time and in addition irradiated cylindrical hohlraums having unit aspect ratios; both types of hohlraums were irradiated with and without fuel capsules. All the hohlraums were fitted with a new diagnostic called a witness plate, which allows us to infer the nature of the preheat on the fuel capsule. The witness plate is described in detail in a subsequent section.

The spherical and cylindrical hohlraums were $\mathbf{2 5 0 0}$ and $2000 \mu \mathrm{m}$ in diam, respectively. When irradiated by an $8-\mathrm{kJ} / 900-\mathrm{ps}$ pulse from the Shiva laser, these hohlraums produced $x$-ray drives of $T_{R}=135$ to $140 \mathrm{eV}$ and preheat fractions of $\mathrm{f}_{\text {hot }}=3$ to $5 \%$. These conditions are Nova-like in the sense that the principal concerns to target designers are radiation physics and hydrodynamics. For $f_{\text {hot }}$ less than $5 \%$, suprathermal-electron physics, although still important, is not the dominant issue in predicting the performance of fuel capsules in the hohlraums.

The environment of an NPIRE hohlraum differs from that of a proposed Nova hohlraum principally in the temporal shape and duration of the radiation drive. An NPIRE hohlraum produces only about $0.5 \mathrm{~ns}$ of peak drive as opposed to the shaped 5-ns peak drive we hope to produce with Nova-like hohlraums.

The fuel capsule and hohlraum issues we wanted to examine with this series are

- Drive and preheat levels in the hohlraums with and without a fuel capsule.

- Relative amounts of x-ray preheat, suprathermal-electron preheat, and thermal x-ray drive.

- Fuel-capsule performance (neutron yield). As explained above, the second issue will be investigated using the witness-plate diagnostic.

NPIRE Fuel Capsules. Fuel capsules were incorporated with the NPIRE hohlraum to answer two questions. Will the capsules and their support structures change the drive and preheat of the hohlraum? Will the capsules perform in a calculable fashion? If the main drive results from radiation, then LASNEX should be able to model the yield and density.

The primary constraint in designing the capsules is that they should be ablatively driven and buildable. These constraints mean that our design would be a glass mandrel having a plastic ablator.

We chose a capsule $(200-\mu \mathrm{m} \times 5-\mu \mathrm{m}+$ $20-\mu \mathrm{m} \mathrm{CH}$ ) filled with $0.01 \mathrm{~g} / \mathrm{cm}^{3}$ of D-T gas (Fig. 4-34). The nominal calculated performance of these targets with a $140-\mathrm{eV} /$ 900-ps drive (Ref. 24) and assuming diffusion transport is

Peak density $\rho_{\max }=14 \mathrm{~g} / \mathrm{cm}^{3}$.

Volume-averaged peak density $\bar{\rho}=10 \mathrm{~g} / \mathrm{cm}^{3}$.

Neutron yield $\mathrm{N}_{\mathrm{y}}=2 \times 10^{8}$.

Assuming PBINTR transport (angular distribution effects included), the nominal performance is

Peak density $\rho_{\max }=9 \mathrm{~g} / \mathrm{cm}^{3}$.

Volume-averaged peak density $\bar{\rho}=6 \mathrm{~g} / \mathrm{cm}^{3}$.

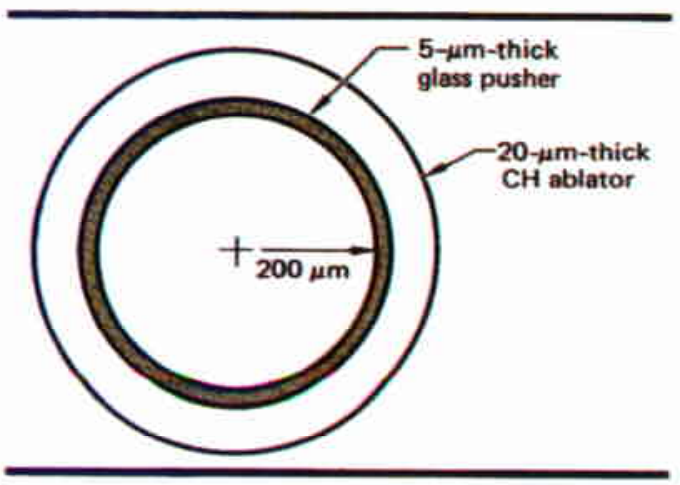

Fig. 4-34. NPIRE fuel capsule. 
Neutron yield $\mathrm{N}_{\mathrm{y}}=3 \times 10^{7}$.

In addition to simulating capsule performance with a nominal hohlraum model, we also explored several other possible hohlraum environments. Our motivation for making these calculations was our concern about $x$ rays in the 2- to $3-\mathrm{keV}$ range and the sensitivity of the capsules to $x$-ray preheat. Below $2 \mathrm{keV}$, the 20- $\mu$ m-thick $\mathrm{CH}$ ablator absorbs very well. Above $2 \mathrm{keV}$ it starts to transmit $x$ rays that are then stopped by the glass, which is thus Table 4-15. NPIRE capsules LASNEX predictions. ${ }^{a}$

\begin{tabular}{|c|c|c|c|}
\hline $\begin{array}{l}\text { Radiation } \\
\text { source }\end{array}$ & $\begin{array}{l}\text { Peak fuel } \\
\text { density } \\
\left(\mathrm{g} / \mathrm{cm}^{3}\right)\end{array}$ & $\begin{array}{l}\text { Radiation energy } \\
\text { density in drive } \\
\text { spectrum, } 0.4 \mathrm{keV} \\
\left.0 / \mathrm{cm}^{3}-\mathrm{keV}\right)\end{array}$ & $\begin{array}{c}\text { Radiation energy } \\
\text { density in preheat } \\
\text { spectrum, } 2.4 \mathrm{keV} \\
\left(0 / \mathrm{cm}^{3}-\mathrm{keV}\right)\end{array}$ \\
\hline Blackbody & 19 & $6 \times 10^{-6}$ & $5 \times 10^{-10}$ \\
\hline $\begin{array}{l}\text { Nominal LASNEX } \\
\text { model }\end{array}$ & 14 & $7 \times 10^{-}$ & $7 \times 10^{-8}$ \\
\hline $\begin{array}{l}\text { High } x \text {-ray preheat } \\
\text { model }\end{array}$ & 4 & $5 \times 10^{-6}$ & $5 \times 10^{-7}$ \\
\hline
\end{tabular}

"All three models would be called " $140-\mathrm{eV}$ sources." However, capsule performance could be severely degraded if energy in preheat spectrum is greater than predicted by our nominal model.

Fig. 4-35. Neutron yield vs $\mathrm{T}_{\mathrm{R}} ; 200-\mu \mathrm{m} \times 5-\mu \mathrm{m}$ $+20-\mu \mathrm{m}$ CH capsule with $0.01 \mathrm{~g} / \mathrm{cm}^{3}$ fill.

Fig. 4-36. Neutron yield and density vs hot-electron preheat using a 200- $\mu \mathrm{m} \times 5-\mu \mathrm{m}+$

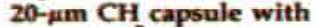
$0.01 \mathrm{~g} / \mathrm{cm}^{3} \mathrm{D}-\mathrm{T}$ fill.

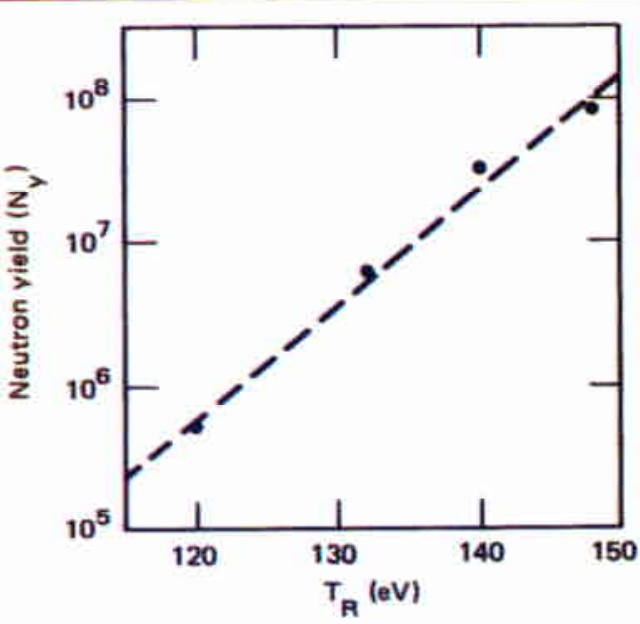

$T_{p}$ was varied by changing the source FDS multiplier in our nominal one-dimensional hohlraum model (see Ref. 24). All calculations used PBINTR transport and $400 \mathrm{~J}$ of $50-\mathrm{keV}$ hot electrons generated at the hohlraum wall.
Table 4-15 contains some of the spectra that we used to model the implosion. These spectra show that severe $\mathrm{x}$-ray preheat can degrade our target. If the 2-to-5-keV spectrum in our hohlraum is about 10 times greater than our nominal non-LTE model indicates, then the implosion will be much different than expected.

$\mathrm{X}$-ray preheat can degrade the density for at least two reasons. First, the stagnation pressure of the hydro system is reduced by preheat. Fuel-less diffusion-transport calculations indicate that without hot electrons the stagnation pressure of a $200-\mu \mathrm{m} \times$ $5-\mu \mathrm{m}+20-\mu \mathrm{m} \mathrm{CH}$ capsule-implosion system is $5 \mathrm{~kJ} / \mathrm{cm}^{3}$. If the amount of hot electrons is $3 \%$, the stagnation pressure is lowered to about $2.2 \mathrm{~kJ} / \mathrm{cm}^{3}$. The high $x$-ray preheat model lowers the peak pressure to about $0.5 \mathrm{~kJ} / \mathrm{cm}^{3}$.

The second reason that high $\mathrm{x}$-ray preheat degrades capsule performance is that it produces too much shock preheat of the fuel. X-ray preheat causes the pusher to swell, which means that the ablatively driven shock breaks out later at the fuel interface since the shock speed is lower in a less-dense material. Thus this shock continues being driven as it passes through the swollen glass (which is strongly designed to break out before the peak of $T_{R}$ ). This extra driving force gives the shock a higher than desired velocity in the absence of swelling. The higher jump-off velocity shocks the fuel to a higher adiabat, which reduces the density.

Because of our concerns about the effects of preheat spectrum, we developed the witness plate in the NPIRE experiment. This diagnostic should help us unravel the performance of the capsule.

Besides the high $x$-ray environments, we have also modeled the capsules in a variety of other possible hohlraums. Figures $4-35$ to

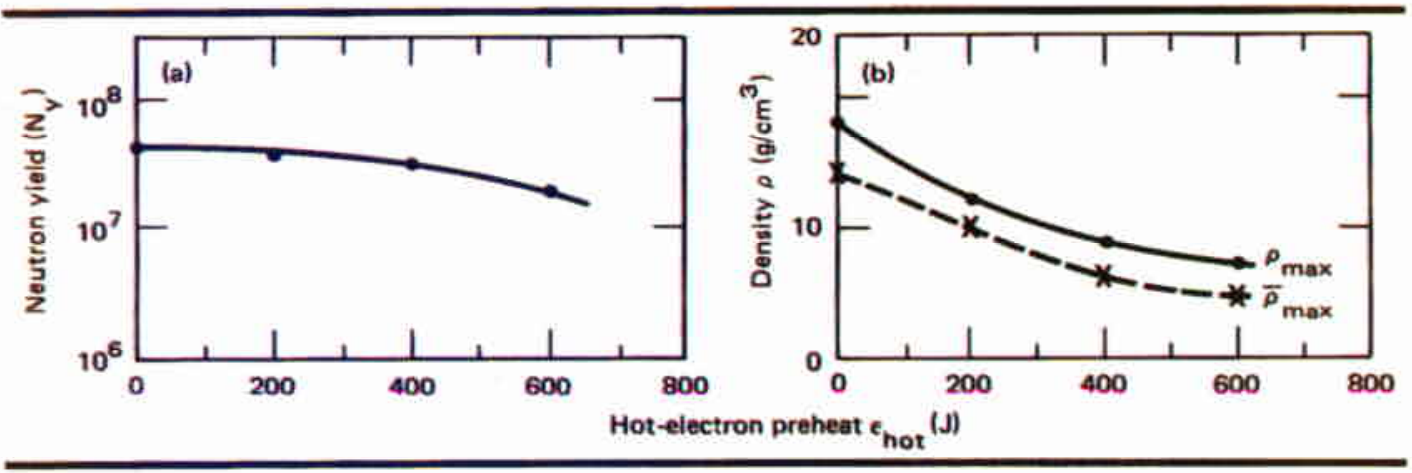


4-37 summarize capsule performance as we culations we used PBINTR radiation transport (which reduces diffusion transport yields by about 10 and density by about 1.5).

Figure 4-35 shows the neutron yield as a function of drive temperature and Fig. 4-36 shows the effect of hot-electron preheat on density yield. Figure 4-37 shows that, in these one-dimensional calculations, scattered laser light that strikes the capsule increases the yield and density. The figure indicates that it is possible for this scattered light (glint) incident on the capsule to compensate for reduced thermal drive. For example, if $T_{R}=120 \mathrm{eV}$ and if $10 \%$ of the laser light strikes the capsule directly, then we would obtain about the same density and yield as we calculate assuming a 140 -eV source.

Figure 4-38 shows fuel density vs neutron-averaged $\rho \Delta R$ of the pusher. The data shown on this graph were taken from calculations where we varied $f_{\text {hot }} T_{k}$, $f_{\text {glint }}$, and $x$-ray preheat. The encouraging aspect of these data is that pusher $p \Delta R$ seems to be a good diagnostic of the peak densities of the capsule. changed the hohlraum. For all of these cal-

When we get additional $\rho \Delta R$ data on future NPIRE experiments, we hope to have a sufficiently complete set of data to allow us to understand the drive and preheat generated in NPIRE hohiraums.

Witness-Plate Diagnostic. Figure 4-39 illustrates the principle of operation of the
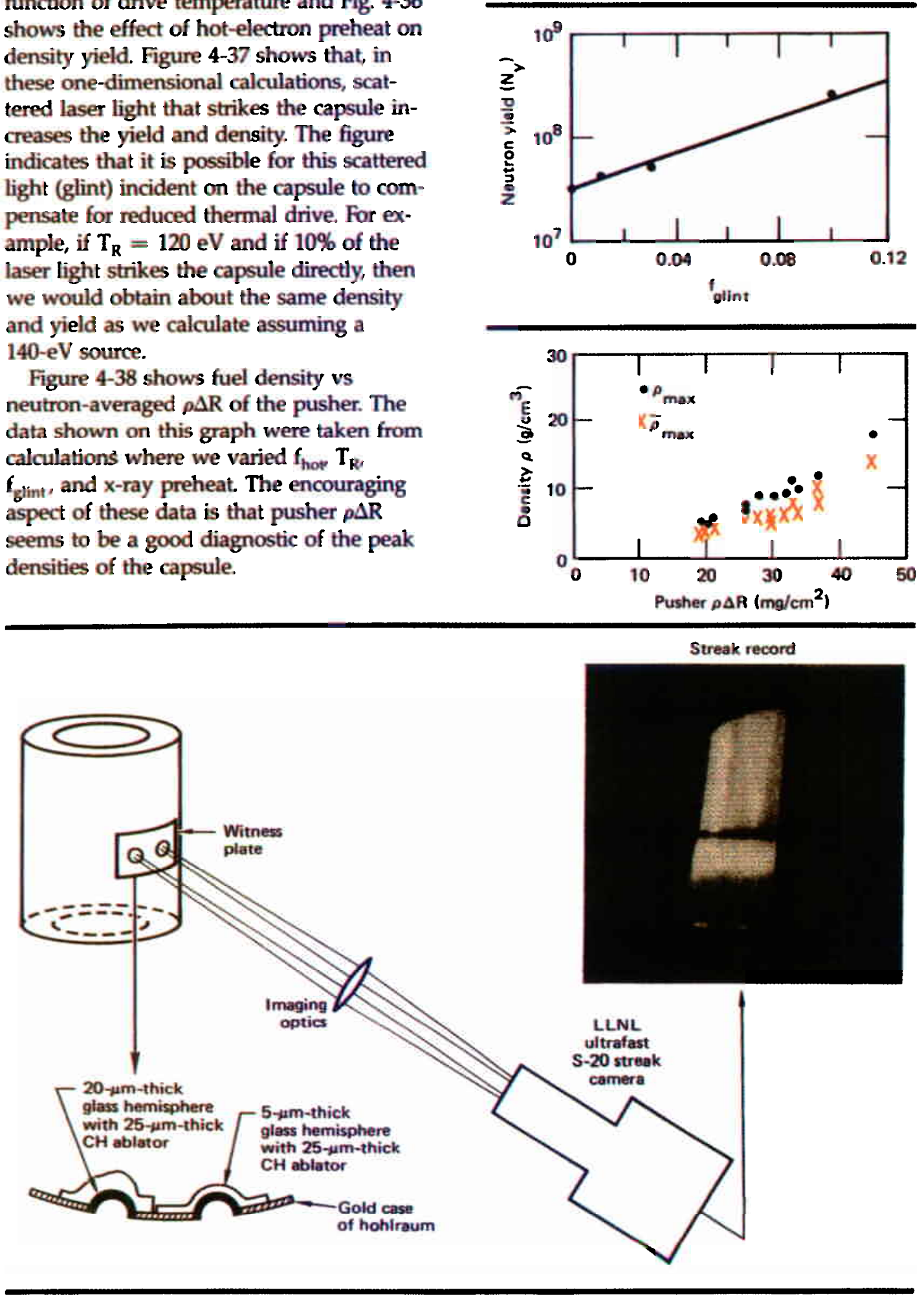

Fig 4-37. Yield vs $f_{\text {elin, the fraction of }}$ incident laser light directly absorbed by the ball.

Fig 4-38. Peak density $\left(\varphi_{\max }\right)$ and peak average density (p) vs neutron-averaged pusher $\rho \Delta R$ for NPIRE capanies.

Fig 4-39. NPIRE experiments; witnessplate diagnostic for preheat messurement. 
witness-plate diagnostic. The witness plate is simply a section of fuel-capsule material mounted on the wall of a hohlraum. As shown in the lower left section of Fig. 4-39, the witness plates in the NPIRE experiments are two glass hemispheres (5 to $20 \mu \mathrm{m}$ thick) with $25 \mu \mathrm{m}$ of $\mathrm{CH}$ ablator. The glass/ablator combination is similar to that of an NPIRE fuel capsule. Hence a diagnostic set up to view the witness plate observes quantities representing the capsule preheat.

An LLNL S-20 optical streak camera was used to record optical emission from the witness plate. As shown in Fig. 4-39, the camera produces a streak record of the emission from two samples. For the NPIRE experiments, the following two samples were used:

- A capsule with a $140-\mu \mathrm{m}$ radius, 5 - $\mu \mathrm{m}$-thick glass wall, and 25 - $\mu \mathrm{m}$-thick $\mathrm{CH}$ ablator.

- A capsule with a $140-\mu \mathrm{m}$ radius,

Fig. 4-40. Each witness plate should give 1/2-Cairn-type temperature signals; first preheat, then shock breakout.

Fig. 4-41. Each graph shows the temperature vs time for two witness plates.

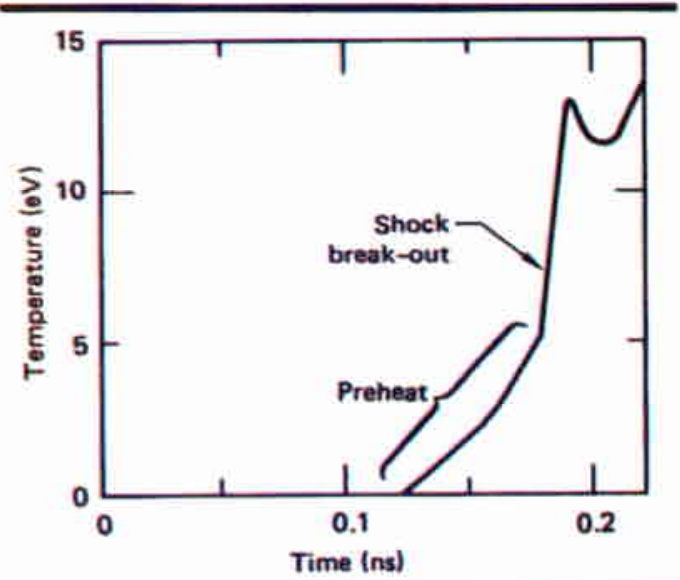

20 - $\mu \mathrm{m}$-thick glass wall, and $25-\mu \mathrm{m}$-thick $\mathrm{CH}$ ablator.

The profile of a streak record has the two main features, as shown in Fig. 4-40. There is an initial preheat region followed by a rapid jump in intensity, which corresponds to the arrival of the shock wave at the rear of the witness plate. This shock wave is produced when the thermal $x$-ray drive ablates the ablator and pusher materials. The shape of the preheat region of the profile is a function of the relative contributions of $x$-ray and suprathermal electron preheat.

We use samples of two different thicknesses to allow relatively unambiguous separation of hot-electron preheat from $x$-ray preheat. That is important information for a target designer, since designing for $x$-ray preheat is different than designing for suprathermal-electron preheat.

Figure 4-41(a) through (c) shows how two plates can be a relatively sensitive diagnostic of preheat as well as drive. Each graph shows the back-surface temperature for two thicknesses of witness plates: 3 and $20 \mu \mathrm{m}$. In all of the calculations the plates are backed by $25 \mu \mathrm{m}$ of $\mathrm{CH}$.

The variable in the three simulations of Fig. 4-41 is the hohlraum conditions. Figure 4-41(a) shows the results for a model having $T_{R}=142 \mathrm{eV}$ and $\mathrm{f}_{\text {hot }}=3 \%$ at $50 \mathrm{keV}$. To get the data for Fig. 4-41(b), we eliminated the hot electrons but kept the radiation the same as in Fig. 4-41(a). Figure 4-41(c) simulated a hohlraum having $3 \%$ hot electrons at $50 \mathrm{keV}$ and a radiation

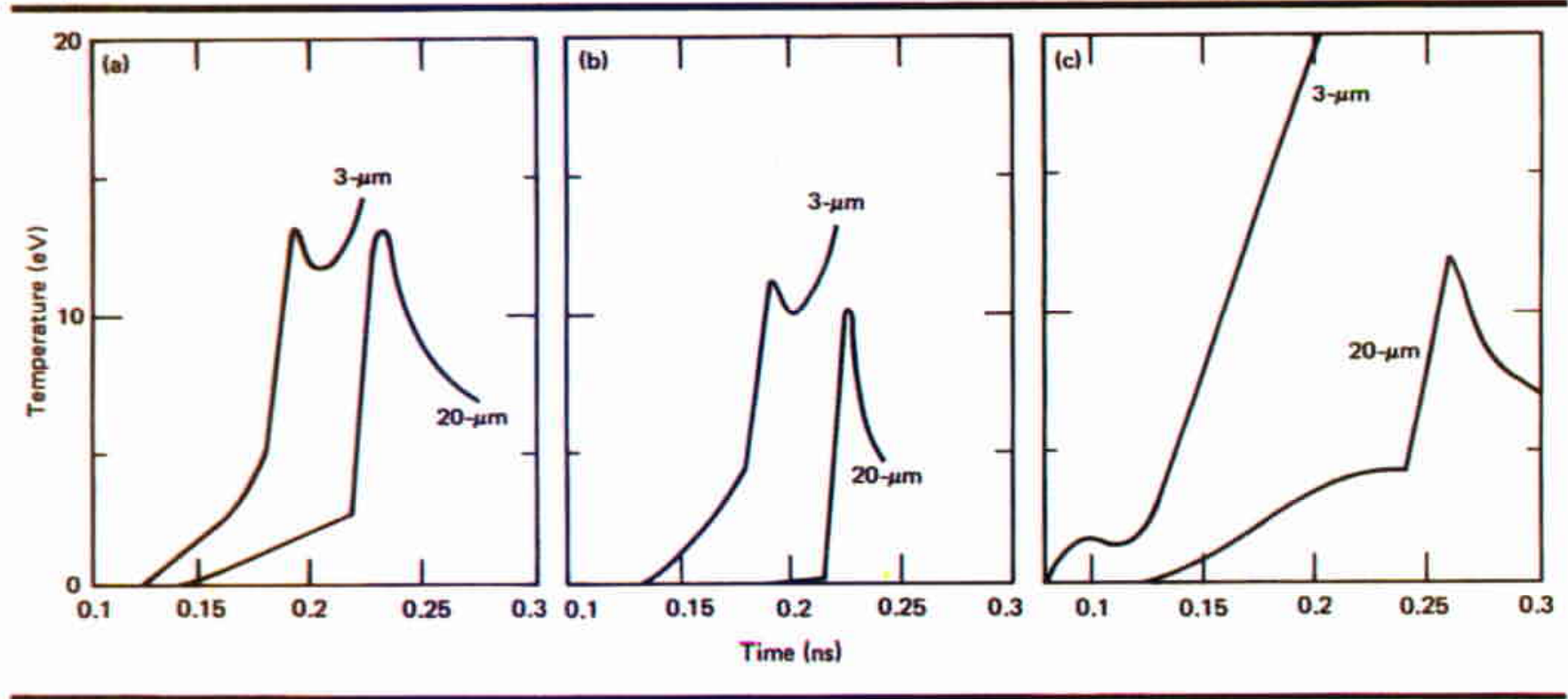


model that greatly increases the "preheat $x$ rays" between 1 and $3 \mathrm{keV}$. However, it would also be described as a $142-\mathrm{eV}$ source.

Figure 4-41 shows that by comparing the temporal dependence of signals from two different plates, we can get a good idea of what is going on in the hohlraum. For example, if drive and hot electrons are about as we expect, then the 5- and 20- $\mu \mathrm{m}$ plates should show a fair amount of preheat, followed by shock breakout.

However, if the amount of hot electrons is low and the radiation is about as we expected, then the results should be like those in Fig. 4-41(b). The thin plate is $x$-ray preheated, while the thicker one remains cool until the shock breaks out.

Finally, if our radiation model is incorrect and there are $\mathbf{a}$ lot of preheat $x$ rays (from, say, gold lines at $2.5 \mathrm{keV}$ ), then we might have results similar to Fig. 4-41(c) in which

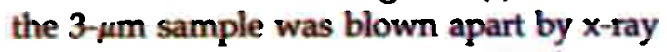
preheat and there was no clear shock breakout. By comparison, the back of the 20 -um sample is preheated primarily by hot electrons and somewhat by $x$ rays. Nevertheless, shock breakout is clearly defined in this sample.

Data similar to those of Fig. 4-41 should help us understand target performance and sort out the physical effects in various hohlraum systems.

Figure 4-42 shows the witness-plate data from one of the spherical hohlraums. The data are currently under analysis. It is important to note that if the spatial distributions of suprathermal electrons and $x$ rays are not uniform, the witness plake will not exactly indicate the preheat conditions at the fuel capsule.

NPIRE Hohlraum Construction. Figure 4-43(a) illustrates the 5/8-4.0 Caim hohlraum. Four targets were built, two contained $200-\mu \mathrm{m} \times 5-\mu \mathrm{m}+20-\mu \mathrm{m} \mathrm{CH}$ fuel capsules. The cylindrical hohlraum is made of gold 15 to $20 \mu \mathrm{m}$ thick, is $2000 \mu \mathrm{m}$ in diam, $2000 \mu \mathrm{m}$ long, and has laser entrance holes $900 \mu \mathrm{m}$ in diam. At the mid-length of the cylindrical surface, two $400-\mu \mathrm{m}$-diam holes are drilled diametrically opposite each other to serve as viewing apertures for the Dante spectrometer and 22X Wolter $x$-ray microscope. (Two holes are required to give the $x$-ray microscope a-dark background.) The witness-plate samples are mounted slightly above the midplane of the cylinder

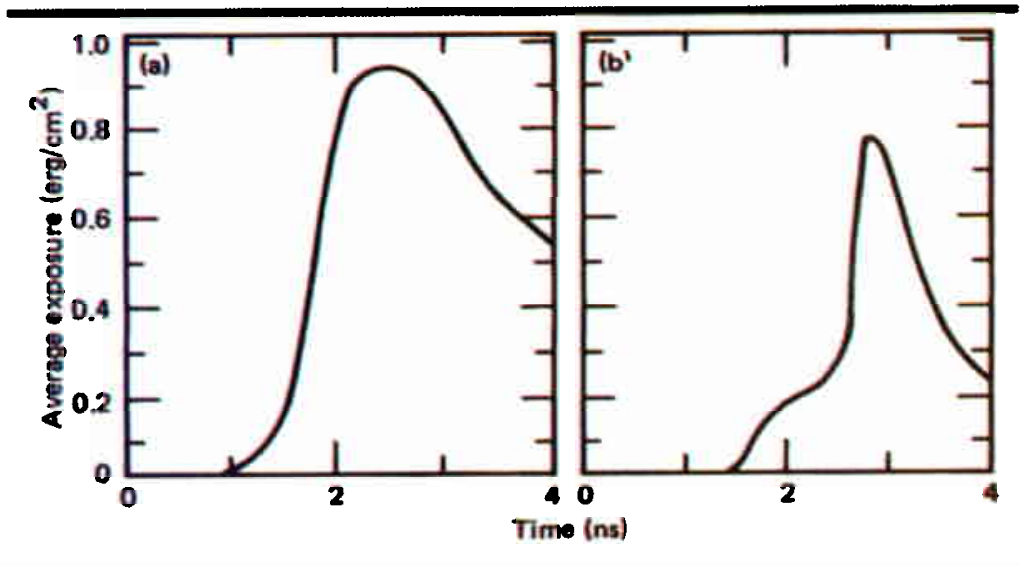

Fig. 4-42. NPIRE experiments; witnessplate data for spherical hohlrawn. (a)

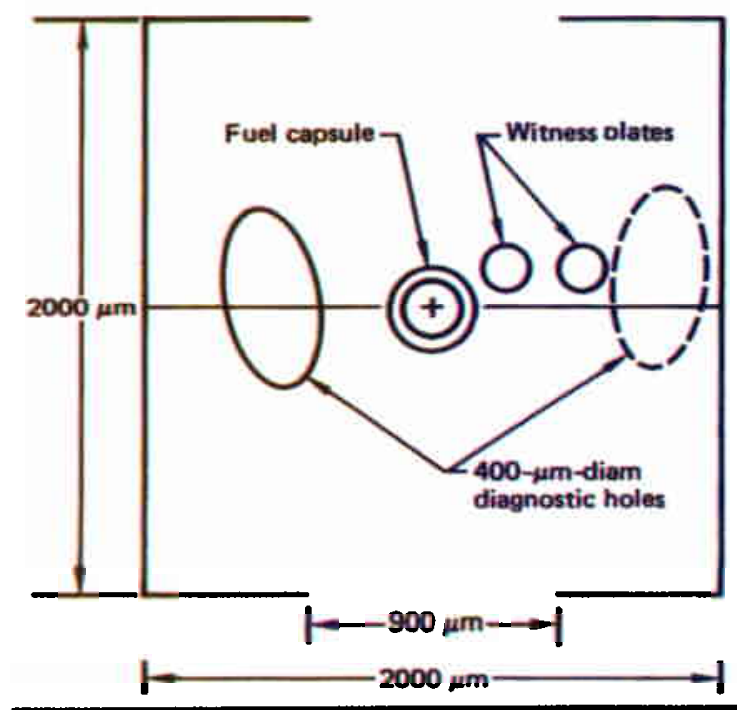

(b)

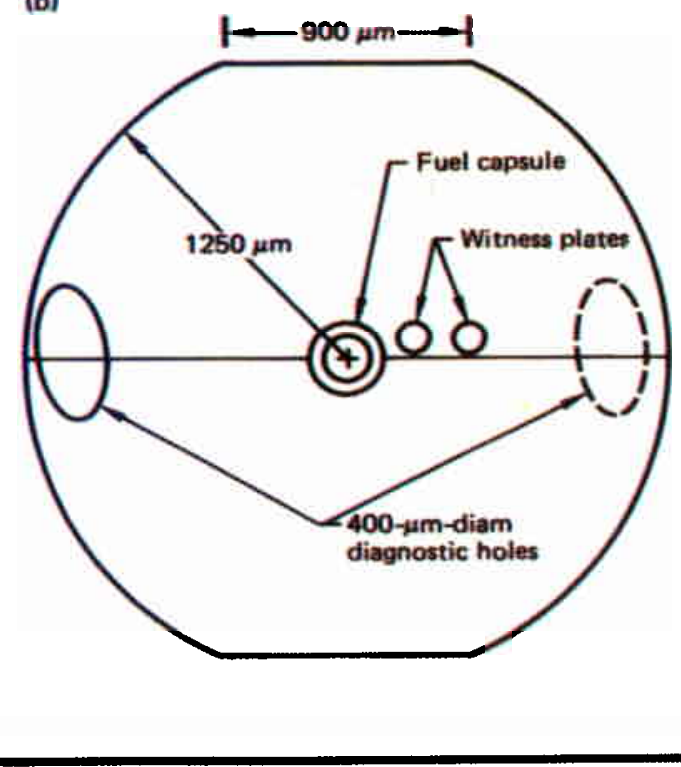

Fig. 4-43. NPIRE hohlraums: (a) 5/8-4.0 E cylinder and (b) 25-S sphere. 
to prevent interference from Formvar sheets that support the fuel capsule.

Figure 4-43(b) shows the 2.5-S spherical hohlraum used in the experiments. The $20-\mu \mathrm{m}$-thick gold hohlraum is $2500-\mu \mathrm{m}$ in diam, has a 900- $\mu \mathrm{m}$-diam laser entrance hole, and has two $400-\mu \mathrm{m}$-diam holes at the equator for viewing the interior with two Dante spectrometers and a $22 \times$ Wolter $x$-ray microscope. As with the 5/8-4.0 Caim hohlraum, two witness-plate samples are mounted slightly above the midplane of the sphere. Each fuel capsule is supported in the hohiraum by two 500-A-thick Formvar films.

The laser beams are aligned to irradiate the hohlraums using the patterns shown in Figs. 4-44 and 4-45; these figures indicate all necessary alignment information. For each alignment pattern, one inner and one outer beam is shown for each cluster of Shiva

ment of Shiva beams into 5/8-4.0 cylindrical hohlraum.
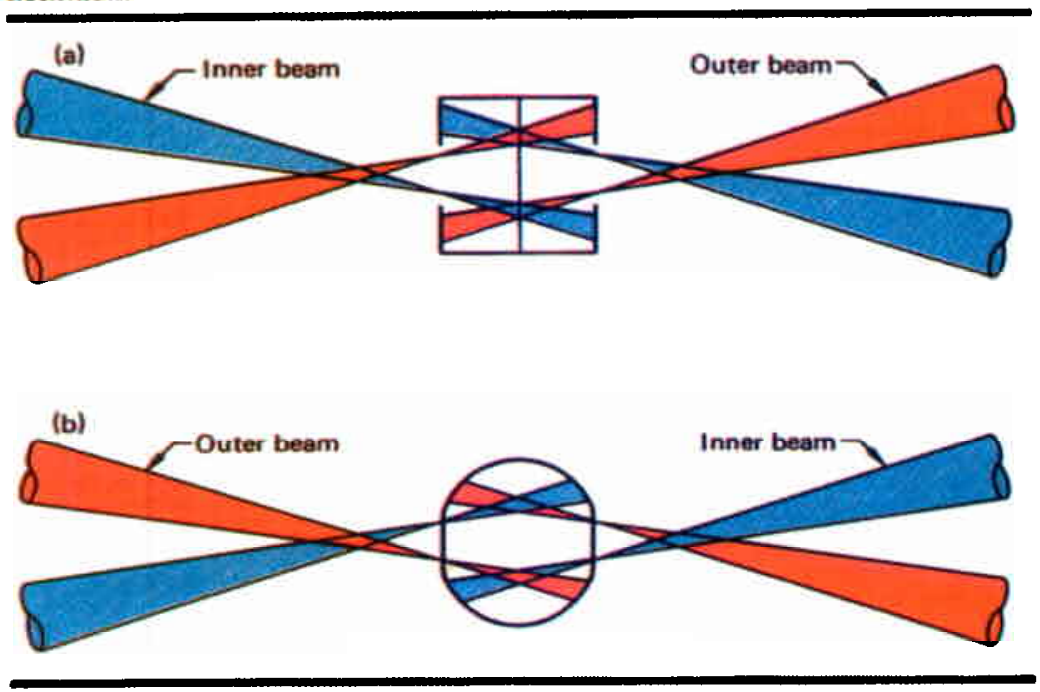

Fig, 4-45. Alignment of Shiva beams into 2.5-S spherical hohlraum.
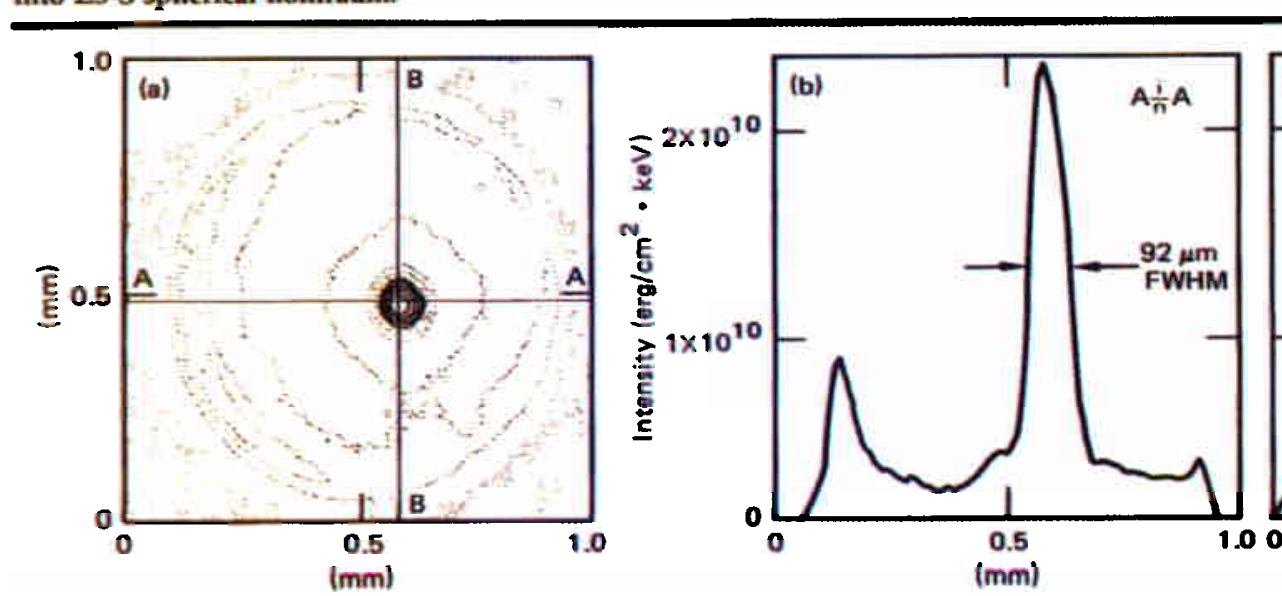

beams. The 10 beams in each cluster are spaced uniformly around the axis of the target at intervals of $36^{\circ}$.

Diagnostics. The diagnostics used for the NPIRE experiments were chosen to characterize the following:

- Absorption of laser light by the hohlraum. - Thermal $x$-ray drive.

- Preheat.

- Fuel-capsule performance (neutron yield and implosion quality).

Hohlraum absorption was determined from the laser energy incident on the hohlraum and the light scattered out of the hohlraum, as measured by arrays of scattered-light photodiodes and laser calorimeters.

The thermal $\mathbf{x}$-ray drive was inferred first from spectral data recorded by two Dante low-energy spectrometers. The S system uses $10 \mathrm{~K}$-edge filter channels and provides spectral coverage from 0.3 to $1.6 \mathrm{keV}$. The $M$ system uses 6 mirror-filter channels and provides spectral coverage from $\mathbf{0 . 2}$ to $1.6 \mathrm{keV}$. Both instruments produce timeresolved data. The time response of the detectors is deconvolved from the data to produce the final temporal histories of the $\mathbf{x}$-ray spectra. A soft $\mathbf{x}$-ray streak camera that provided time-resolved spectra with 15-ps resolution was also used in the experiments.

The levels of preheat resulting from suprathermal electrons and $x$ rays were measured with three diagnostics:

- A filter-fluorescer high-energy spectrometer that covers the spectrum from $4 \mathrm{keV}$ to $300 \mathrm{eV}$. (The spectrometer measures the time-averaged bremsstrahlung emission from the suprathermal electrons; the 
suprathermal-electron temperature and $\mathbf{f}_{\text {ho }}$ are inferred from the characteristics of the bremsstrahlung measurements.)

- A crystal spectrometer that measures the $x$-ray spectral range of 2 to $5 \mathrm{keV}$ (which is relevant to $x$-ray preheat).

- An optical pyrometer that obtains time histories of the preheat levels in the witness-plate samples.

The time history of the high-energy $\mathbf{x}$ rays was recorded with two streak cameras: the LLNL $x$-ray streak camera (which provided coverage up to $25 \mathrm{keV}$ ) and the optical/ x-ray interval streak camera (which provided data on the relative timing between the 1.06- $\mu \mathrm{m}$ laser pulse and the $x$-ray emission for photons with energy $\geq 30 \mathrm{keV}$ ). This was accomplished by placing a sheet of lead over half of the slit of an S-1 optical streak camera. The streak tube thus received laser light and $x$ rays on half of its area but only $x$ rays on the other half. The laser-light contribution is many times stronger than the $x$-ray contribution to the combined signal on one side. An $x$-ray pinhole camera recorded the spatial distribution $\mathbf{x}$ rays from the hohlraum having energies $\geq 8 \mathrm{keV}$. This instrument recorded the location of any localized intense sources of $\dot{x}$-ray emission.

In this series the principal variable for characterizing fuel-capsule performance was neutron yield. Future experiments will be directed toward using a fuel-density measurement as an additional indicator of capsule performance. Neutron yield was

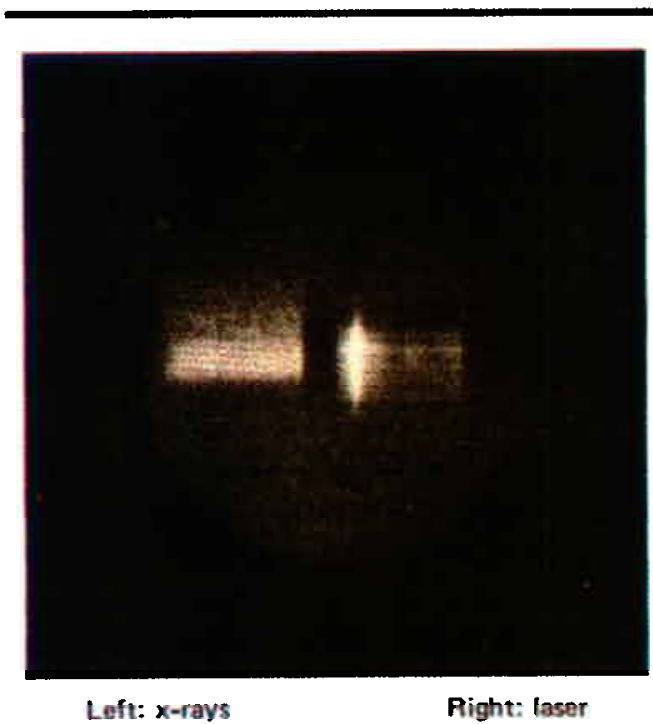

Fig. 4-46, Streak record from optical/x-ray interval streak camera.

\begin{tabular}{|c|c|c|c|c|c|c|c|c|c|c|}
\hline \multirow{3}{*}{$\begin{array}{c}\text { Shot } \\
\text { number }\end{array}$} & & & \multicolumn{3}{|c|}{ Laser performance } & \multirow{3}{*}{$\begin{array}{c}\text { Neutron } \\
\text { yieid }\end{array}$} & \multirow{3}{*}{$\begin{array}{c}\text { Absorption } \\
(\%)\end{array}$} & \multirow{2}{*}{\multicolumn{3}{|c|}{ Thermnal drive, $T_{R}(\mathrm{eV})$}} \\
\hline & \multicolumn{2}{|c|}{ Tanget data } & \multirow{2}{*}{$\begin{array}{l}\text { Pulse } \\
\text { (kJ/ns) }\end{array}$} & \multicolumn{2}{|c|}{ Beams (k) } & & & & & \\
\hline & Type & Scale & & $1-10$ & $11-20$ & & & Dante-S & Dante-M & $\mathrm{SX}-2$ \\
\hline 80120304 & Caim 267" & $5 / 8-4.0 \mathrm{E2}$ & $732 / 900$ & 3.73 & 3.59 & & $62 \pm 10$ & 122 & 131 & 146 \\
\hline 80120405 & Caim $265^{b}$ & $5 / 8-4.0 \mathrm{E2}$ & $9.93 / 838$ & 4.74 & 5.19 & $(4.2 \pm 0.7) \times 10^{6 c}$ & $84 \pm 10$ & & 133 & 140 \\
\hline 80120410 & Caim $266^{d}$ & $5 / 8-4.0 \mathrm{E2}$ & $9.08 / 942$ & 4.54 & 4.54 & $(3.4 \pm 0.2) \times 10^{6 c}$ & $\pi 5 \pm 10$ & 129 & 136 & \\
\hline 80120606 & $\mathrm{SH}-11^{\mathrm{e}}$ & $25 S 2$ & $9.09 / 900$ & 4.55 & 4.54 & $(3.8 \pm 0.3) \times 10^{7 f}$ & & 131 & 137 & \\
\hline 80120603 & $\mathrm{SH}-128$ & 2552 & $8.69 / 897$ & 4.41 & 4.28 & $(1.1 \pm 0.2) \times 10^{7 c}$ & $83 \pm 10$ & 131 & 133 & \\
\hline 80120806 & $\mathrm{SH}-10^{2}$ & 2.552 & $8.36 / 897$ & 3.96 & 4.40 & & $76 \pm 10$ & 138 & 139 & \\
\hline 80120909 & SH-13 & 2552 & $8.77 / 900$ & 4.34 & 4.43 & & $73 \pm 10$ & 132 & 138 & \\
\hline \multirow[t]{2}{*}{80121004} & Cairn $264^{\mathrm{a}}$ & $5 / 8-4.0 \mathrm{E2}$ & $8.51 / 900$ & 4.40 & 4.11 & & & 128 & 134 & \\
\hline & \multicolumn{5}{|c|}{ FFLEX data (keV/keV) } & \multicolumn{3}{|c|}{ Raman spectra } & & \\
\hline $\begin{array}{c}\text { Shot } \\
\text { number }\end{array}$ & \multicolumn{2}{|c|}{$\begin{array}{r}\mathrm{I}_{50 \mathrm{keV}} \\
\left(\times 0^{13}\right)\end{array}$} & $\begin{array}{l}\mathrm{l}_{84} \mathrm{kev} \\
\left(\times 10^{13}\right)\end{array}$ & & $\begin{array}{l}\mathrm{I}_{200 \mathrm{kgX}} \\
\left(\times 10^{13}\right)\end{array}$ & $\begin{array}{l}\text { peak } \\
(\mu \mathrm{m})\end{array}$ & \multicolumn{2}{|c|}{$\begin{array}{c}\text { Ipeak } \\
0 / \text { sr-pm) }\end{array}$} & & \\
\hline 80120304 & \multicolumn{2}{|c|}{2.67} & 1.95 & & 0.135 & 1.52 & \multicolumn{2}{|c|}{41} & & \\
\hline 80120405 & \multicolumn{2}{|c|}{20.9} & 14.4 & & & $1.86,1.92$ & \multicolumn{2}{|c|}{144} & & \\
\hline 80120410 & \multicolumn{2}{|c|}{15.7} & 10.5 & & 0.721 & $1.69,1.76$ & \multicolumn{2}{|c|}{156} & & \\
\hline 80120505 & \multicolumn{2}{|c|}{27.8} & 15.1 & & 1.62 & 1.82 & \multicolumn{2}{|c|}{104} & & \\
\hline 80120803 & \multicolumn{2}{|c|}{21.4} & 8.93 & & 0.698 & 1.75 & \multicolumn{2}{|c|}{$155^{\mathrm{h}}$} & & \\
\hline 80120806 & \multicolumn{2}{|c|}{3.81} & 1.06 & & 0.0333 & 1.51 & \multicolumn{2}{|c|}{86} & & \\
\hline 80120909 & \multicolumn{2}{|c|}{7.72} & 225 & & 0.124 & 1.48 & \multicolumn{2}{|c|}{144} & & \\
\hline 80121004 & \multicolumn{2}{|c|}{3.09} & 1.42 & & 0.186 & 1.48 & \multicolumn{2}{|c|}{113} & & \\
\hline
\end{tabular}

"Witness plate.

Witness plate, $1885-\mu \mathrm{m} \times 5-\mu \mathrm{m}+18.7 \mathrm{CH}$, D-T density $=10 \mathrm{mg} / \mathrm{cm}^{3}$.

By lead ACT.

${ }^{d}$ Witness plate, $193-\mu \mathrm{m} \times 5-\mu \mathrm{m}+19.5 \mathrm{CH}, \mathrm{D}-\mathrm{T}$ density $=10 \mathrm{mg} / \mathrm{cm}^{3}$.

'Witness plate, $211-\mu \mathrm{m} \times 3.8-\mu \mathrm{m}+19.1 \mathrm{CH}, \mathrm{D}-\mathrm{T}$ density $=10 \mathrm{mg} / \mathrm{cm}^{3}$.

'By copper ACT.

gitness plate, $209.5-\mu \mathrm{m} \times 5.8-\mu \mathrm{m}+19.1 \mathrm{CH}$, D-T density $=10 \mathrm{mg} / \mathrm{cm}^{3}$.

${ }^{h}$ Flat, 1.4-1.7. 
Fig. 4-47. NPIRE experiments; $22 \times$ x-ray microscope records time history of fuel capsule and hohlraum plasma dynamics.

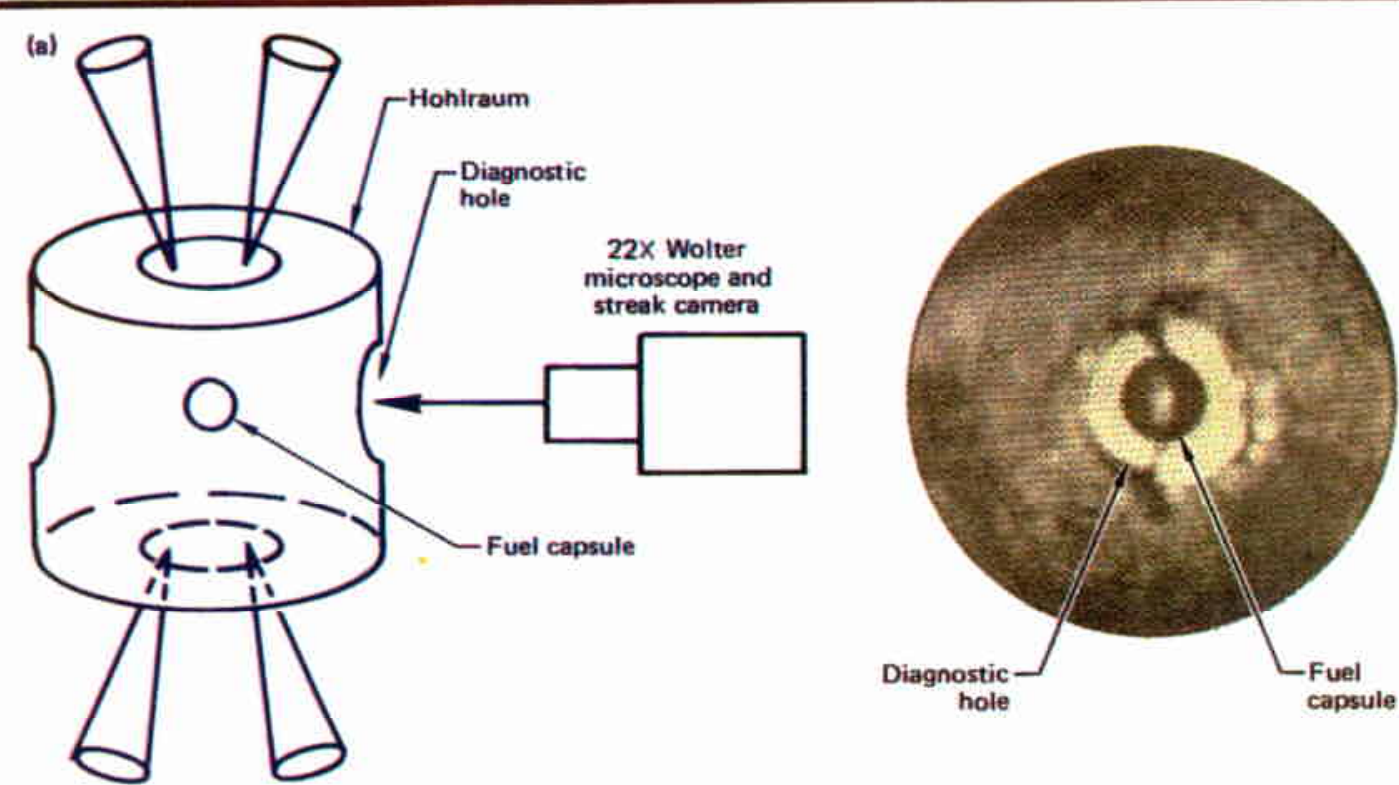

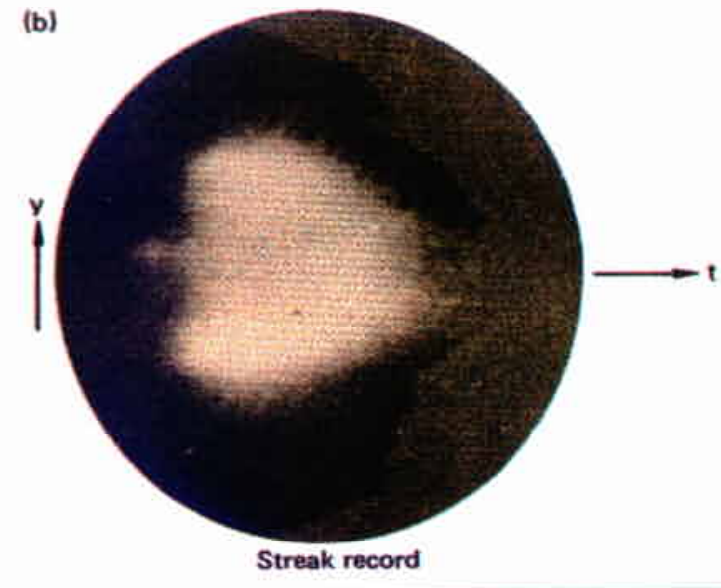

(b)

measured using a lead-activation detector having a threshold of $5 \times 10^{5}$. An $x$-ray zone-plate camera was fielded on three experiments that had fuel-capsule/hohlraum targets; these experiments were attempts to image the $x$-ray emission from the compressed pusher and hence to acquire information on implosion quality and symmetry. None of the zone plate images showed any contribution from pusher $x$-ray emission, so no data on implosion quality were obtained. Future experiments utilizing $x$-ray backlighting will be fielded to obtain these data.

Experimental Results. Eight NPIRE targets were irradiated in December 1980 (Table 4-16). There were four 5/8-4.0 cylindrical hohlraums, two with $200-\mu \mathrm{m} \times 5-\mu \mathrm{m}$ $+20-\mu \mathrm{m} \mathrm{CH}$ fuel capsules and two without. There were also four 2.5-S spherical hohlraums, two with $200-\mu \mathrm{m} \times 5-\mu \mathrm{m}+$ $20-\mu \mathrm{m} \mathrm{CH}$ fuel capsules and two without. The experiments using the spherical hohlraums were the first target experiments having this type of geometry. In addition, the experiments were the first successful tests of the witness-plate diagnostic.

The Shiva laser system was set up to operate at 8 to $10 \mathrm{~kJ}$ of energy using a pulse width of $900 \mathrm{ps}$. The laser system performed flawlessly with all eight targets and a good data package was obtained for each. Authors; J. M. Auerbach and L. J. Suter 
There were seven major results of this highly successful series:

(1) The low-energy $x$-radiation drive $\left(T_{t}\right)$ and hot-electron levels $\left(E_{h}\right)$ scaled from Argus to Shiva, in agreement with scaling laws that had been previously ${ }^{28,29}$ triply derived: analytically, computationally, and empirically.

(2) We measured 5-TPa (50-Mbar) radiation-driven shocks propagating through glass samples. This is a record for a laboratory measurement of high pressure.

(3) We observed (with x-ray backlighting) the motion of a sample radiatively accelerated to a velocity of nearly $2 \times 10^{7}$ $\mathrm{cm} / \mathrm{s}$.

(4) We measured the preheat levels through sample thicknesses relevant to our $100 \mathrm{X}$ target design.

\section{Ablator and Pusher Studies}

\section{Half-Cairn Experiments on Shiva-Introduction}

In recent years, we have successfully performed physics experiments ${ }^{25,26}$ with the Angus laser system to measure the radiation and hot-electron fluences incident on a slab of material inside a laser-driven hohlraum. We also used these experiments to measure the preheat, shock, and hydrodynamic motion on the back side of that slab. We have also been able to analyze the cause-andeffect relationship between these two sets of measurements with a variety of analytic scaling models ${ }^{27}$ and LASNEX simulations. This past year we have performed similar experiments on the Shiva laser system.

They differed from the Argus experiments principally in four areas:

- The higher radiation drive.

- The higher hot-electron levels present in the hohlraum illuminated with Shiva.

- The extra diagnostic capability of $x$-ray backlighting the slab's motion.

- The extra diagnostic capability of detecting 100 -to- $300-\mathrm{keV} \times$ rays.

In this article we discuss the experimental series and analyze the results.
(5) We found evidence for a super-hot tail $\left(\mathrm{T}_{\mathrm{h}} \approx 120 \mathrm{keV}\right)$, which makes its presence known at $x$-ray energies greater than $250 \mathrm{keV}$ (an energy region not probed with Argus diagnostics).

(6) We observed unexpected preheat through 50- $\mu \mathrm{m}$-thick Au samples.

(7) With a two-pronged approach to the analysis of this data, namely, simple analytic models and complex LASNEX simulations, we successfully related the measured causes and effects.

The theory in (7) shows that the drives in (1) indeed lead to the observations (2) through (4), as is the case for the effect (6) being caused by the super-hot tail in observation (5).

The first article that follows, "Half-Cairn Studies," describes the experimental setup and the preheat and shock observations. The second article, "Enterprise SeriesHohlraum Experiments," discusses the x-ray backlighting of the slab in motion as it accelerates out of the hohlraum. In both articles, the data are analyzed in terms of simple models and LASNEX simulations.

Authors: M. D. Rosen, R. H. Price, and D. L. Banner 